\title{
Involvement of platelet cytoskeleton and plasma membrane in the expression of procoagulant activity
}

Citation for published version (APA):

Verhallen, P. F. J. (1988). Involvement of platelet cytoskeleton and plasma membrane in the expression of procoagulant activity. [Doctoral Thesis, Maastricht University]. Rijksuniversiteit Limburg. https://doi.org/10.26481/dis.19881118pv

Document status and date:

Published: 01/01/1988

DOI:

10.26481/dis.19881118pv

Document Version:

Publisher's PDF, also known as Version of record

\section{Please check the document version of this publication:}

- A submitted manuscript is the version of the article upon submission and before peer-review. There can be important differences between the submitted version and the official published version of record.

People interested in the research are advised to contact the author for the final version of the publication, or visit the DOI to the publisher's website.

- The final author version and the galley proof are versions of the publication after peer review.

- The final published version features the final layout of the paper including the volume, issue and page numbers.

Link to publication

\footnotetext{
General rights rights.

- You may freely distribute the URL identifying the publication in the public portal. please follow below link for the End User Agreement:

www.umlib.nl/taverne-license

Take down policy

If you believe that this document breaches copyright please contact us at:

repository@maastrichtuniversity.nl

providing details and we will investigate your claim.
}

Copyright and moral rights for the publications made accessible in the public portal are retained by the authors and/or other copyright owners and it is a condition of accessing publications that users recognise and abide by the legal requirements associated with these

- Users may download and print one copy of any publication from the public portal for the purpose of private study or research.

- You may not further distribute the material or use it for any profit-making activity or commercial gain

If the publication is distributed under the terms of Article $25 \mathrm{fa}$ of the Dutch Copyright Act, indicated by the "Taverne" license above, 
Involvement of platelet cytoskeleton and plasma membrane in the expression of procoagulant activity 


\section{CIP-DATA KONINKLIJKE BIBLIOTHEEK, DEN HAAG}

Verhallen, Petrus Franciscus Jacobus

Involvement of platelet cytoskeleton and plasma membrane in the expression of procoagulant activity / Petrus Franciscus Jacobus Verhallen. - [S.l. : s.n.]. - Il].

Thesis Maastricht. - with ref. - With summary in Dutch.

ISBN 90-9002517-0

SISO 605.19 UDC 612.1 (043.3)

Subject headings: platelets / calpain / procoagulant activity.

(1) 1988. P.F.J. Verhallen.

The contents of this book are free to reproduce in any way without permission of the author, on condition that appropiate reference is made. 


\title{
Involvement of platelet cytoskeleton and plasma membrane in the expression of procoagulant activity
}

Proefschrift

\author{
ter verkrijging van de graad van doctor \\ aan de Rijksuniversiteit Limburg te Maastricht, \\ op gezag van de Rector Magnificus, Prof. Dr. F.I.M. Bonke, \\ volgens het besluit van het College van Dekanen, \\ in het openbaar te verdedigen, \\ op vrijdag 18 november 1988 , om 16.00 uur
}

door

Petrus Franciscus Jacobus Verhallen

geboren te Maastricht

in 1955 


\section{Promotor:}

Prof. Dr. R.F.A. Zwaal

Co-promotor:

Dr. E.M. Bevers

\section{Beoordelingscommissie:}

Prof. Dr. J. Jolles, Rijksuniversiteit Limburg, Maastricht

Prof. Dr. M.C. Scrutton, King's College, London

Prof. Dr. H.A.J. Struyker Boudier, Rijksuniversiteit Limburg, Maastricht Dr. A.J.W.G. Visser, Landbouw Universiteit Wageningen.

Prof. Dr. G.J. van der Vusse, Rijksuniversiteit Limburg, Maastricht

The research described in this thesis was performed at the Department of Biochemistry, in close collaboration with Edlouard Bevers and Paul Confurius, and was financially supported by program grant $900-526-093$ from MEDIGON (The Dutch Foundation for Medical and Health Research).

Financial support by the Netherlands Heart Foundation and the Jan Dekker \& dr. Ludgardine Bouwman Foundation for the publication of this thesis is gratefully acknowledged. 
Life is what you make it Talk Talk

Face it, enjoy it, control it B.Luiwammes

Voor mijn dierbaarsten,

Sylvia

Hugo

Pieter

Aernout 



\section{CONTENTS}

page

$\begin{array}{lll}\text { Chapter } 1 \text { General introduction. } & 1\end{array}$

Chapter $2 \quad$ Platelet procoagulant activity and cytoskeletal degra- $\quad 29$ dation by calpain.

Verhallen et.al. (1987)

Biochim.Biophys_Acta, 903: 206-217.

Chapter 3 Relation between calpain and phospholipid transbilayer movement in fluoride-treated platelets.

Verhallen et.al. (1988)

Biochim.Biophys.Acta, 942: 150-158.

Chapter 4 Phospholipid transbilayer asymmetry in artificially induced platelet plasma membrane vesicles.

Bevers et.al. (1987)

Biochim.Biophys_Acta, 903: 197-205.

Chapter 5 Transbilayer asymmetry of membrane tluidity and cholesterol in unstimulated platelets.

Verhallen et.al, submitted

Chapter 6 Rapid transbilayer movement in the platelet plasmat membrane during cytoskeletal degradation by calpain.

Verhallen et.al., submitted

Chapter 7 General discussion

References

Summary

Samenvatting

Dankwoord

List of publications

Curriculum vitae 


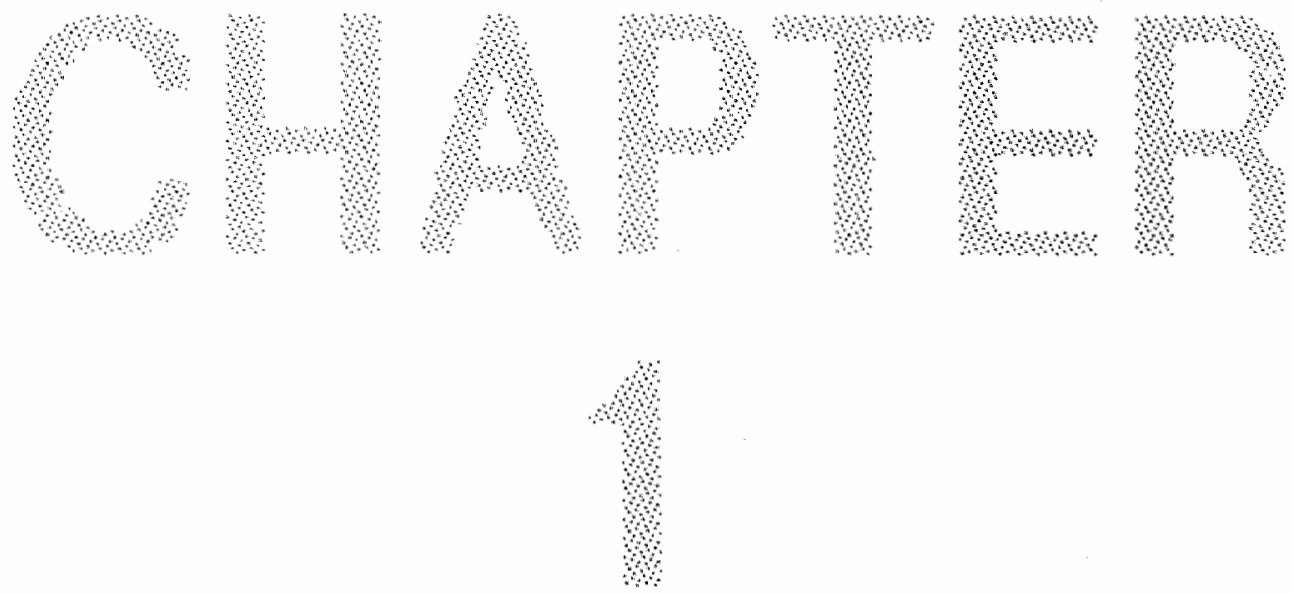



\section{GENERAL INTRODUCTION}

This thesis will deal with cellular and molecular mechanisms which are involved in the loss of transbilayer lipid asymmetry in the platelet plasma membrane when platelets become procoagulant upon activation. As a general introduction, the major subjects of the research decribed in this thesis will be discussed in detail to set the scope of the following chapters. First, the structure and function of human platelets will be covered. Second, the properties of calpain, an endogenous $\mathrm{Ca}^{2+}$-dependent protease, will be discussed. Third, regulation of transbilayer lipid asymmetry in lipid model systems and in the erythrocyte will be addressed. Finally, various aspects of platelet procoagulant activity will be reviewed.

The purpose of this general introduction is not merely to provide sufficient background information to understand the following chapters, but it is also ment to provide adequate depth to place the following chapters in a broader perspective, and to provide detailed entrance to the literature. At the risk of supplying redundant information, I hope it will aid those, interested in but not familiar with the fields of platelet biochemistry, calpain, transbilayer lipid asymmetry, and platelet procoagulant activity. 


\section{STRUCTURE AND FUNCTION OF THE HUMAN PLATELET}

Blood platelets are highly reactive cells which participate in the arrest of bleeding upon vessel wall injury by the formation of a hemostatic plug. Normally, platelets circulate in the blood stream for 8-11 days as quiescent cells. Upon vessel wall injury, blood platelets instantly adhere to the uncovered subiendothelial fibrils, change shape, and secrete their granular contents which in turn results in stimulation of other circulating platelets to aggregate on the adherent layer to form a platelet plug "This primary hemostatic plug will prevent leakage of blood from the damaged vessel in normal hemostasis, or may obstruct the blood stream within the vessel in thrombotic processes and in the pathology of severe atherosclerotic lesions. The primary platelet plug is consolidated by fibrin to form a stable final hemostatic plug. Fibrin is formed upon initiation of the coagulation cascade, a process which is dramatically accelerated when activated platelets become procoagulant. Finally, contractile mechanisms in platelets lead to retraction of the clot.
The platelet has become a well studied cell, and many aspects of cel biology and cellular biochemistry are best undersiood in the platelet. The following subjects will be discussed in more detail: (1) platelet morphology, (2) platelet responses and stimuli, (3) composition and function of the platelet cytoskeleton, (4) structure and composition of the platelet plasma membrane, and (5) platelet signal transduction.

\section{Platelet morphology}

Platelets are anucleate cytoplasmic fragments originating from megakaryocytes in the bone marrow $[524,566]$. They circulate as diskshaped smooth cells, with an equatorial diameter of $2-4 \mu \mathrm{m}$ and a thickness of about $1 \mu \mathrm{m}$. Platelet ultrastructure has been studied extensively by White and co-workers $[694,695,697$, 699]. The main features of platelet morphology are shown in Fig. 1. Based on electron micro-

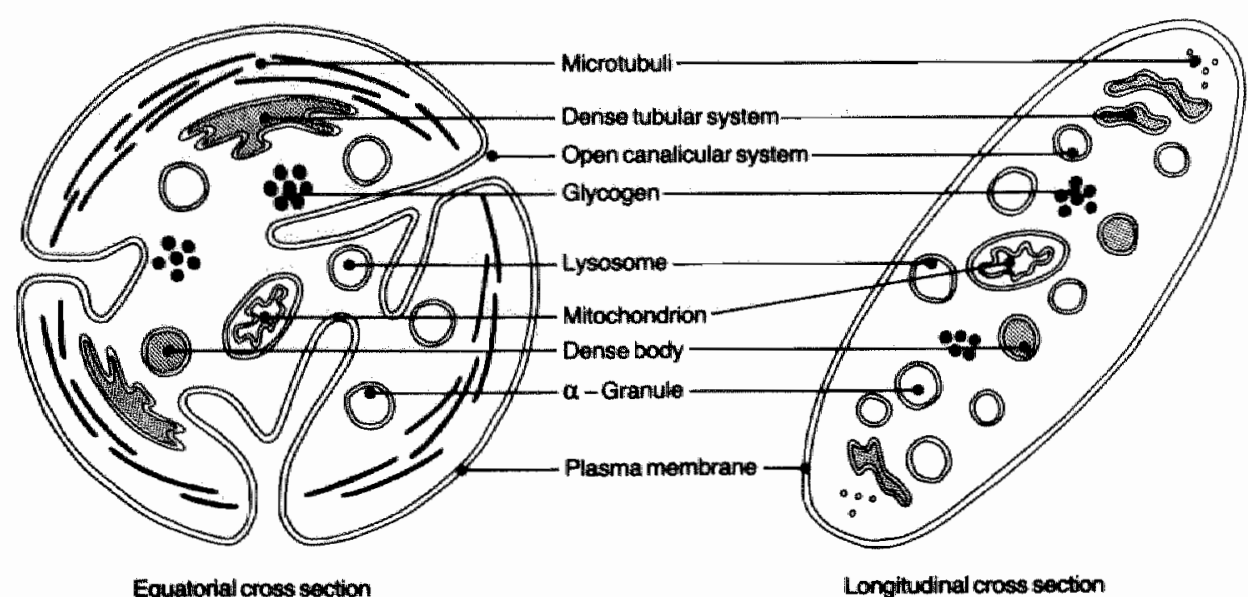

Fig. 1. Morphology of the unstimulated discoid platelet. Schematic representation of structural features as observed by thin-section electron microscopy. The various components are described in the text. 
scopic investigations, the platelet can be diwided into four distinct regions: peripheral zone, sol-gel zone, organelle zone, and internal membrane systems.

The peripheral zone constitutes the platelet plasma membrane, which is extensively inwaginated into the cell interior to form the open canalicular system. The plasma membrane is covered by an exterior coat (glycocalyx), which is thicker and more dense than that of other blood cells. The exterior coat is rich in glycoproteins, which play an important role in various platelet functions. The glycoproteins carry terminal sialic acid residues which confer a large negative surface charge to the platelet. At the cytosolic side of the plasma membrane a system of filamentous elements is found between the membrane and the circumferential band of microtubuli. This filamentous submembrane region will be discussed in more detail as membrane skeleton.
The sol-gel zone consists of a circumferential band of microtubuli, located in the equatorial plane. The microtubule band consists of a single polymer of tubulin, coiled to form 10 to 20 circles [441]. Close to the microtubule band amorphous precursors of the cytoskeleton can be found, which will polymerize into organized cytoskeletal structures (sol-gel transition) during platelet activation.

In the cytoplasm of platelets various secretory vesicles can be detected, which are randomly distributed in unstimulated platelets but become centralized upon activation. The electron-dense bodies contain serotonin and a non-metabolic (storage) pool of $\mathrm{Ca}^{2+}$ ATP, and $\mathrm{ADP}$, in the form of high-molecular weight aggregates [146]. The less dense granules are the most abundant organelles, and can be divided into $\alpha$-granules, lysosomes, and peroxisomes. $\alpha-$ Granules are protein storage sites, which contain a number of non-ezymatic proteins $[308,458]$. Some of these proteins partici-

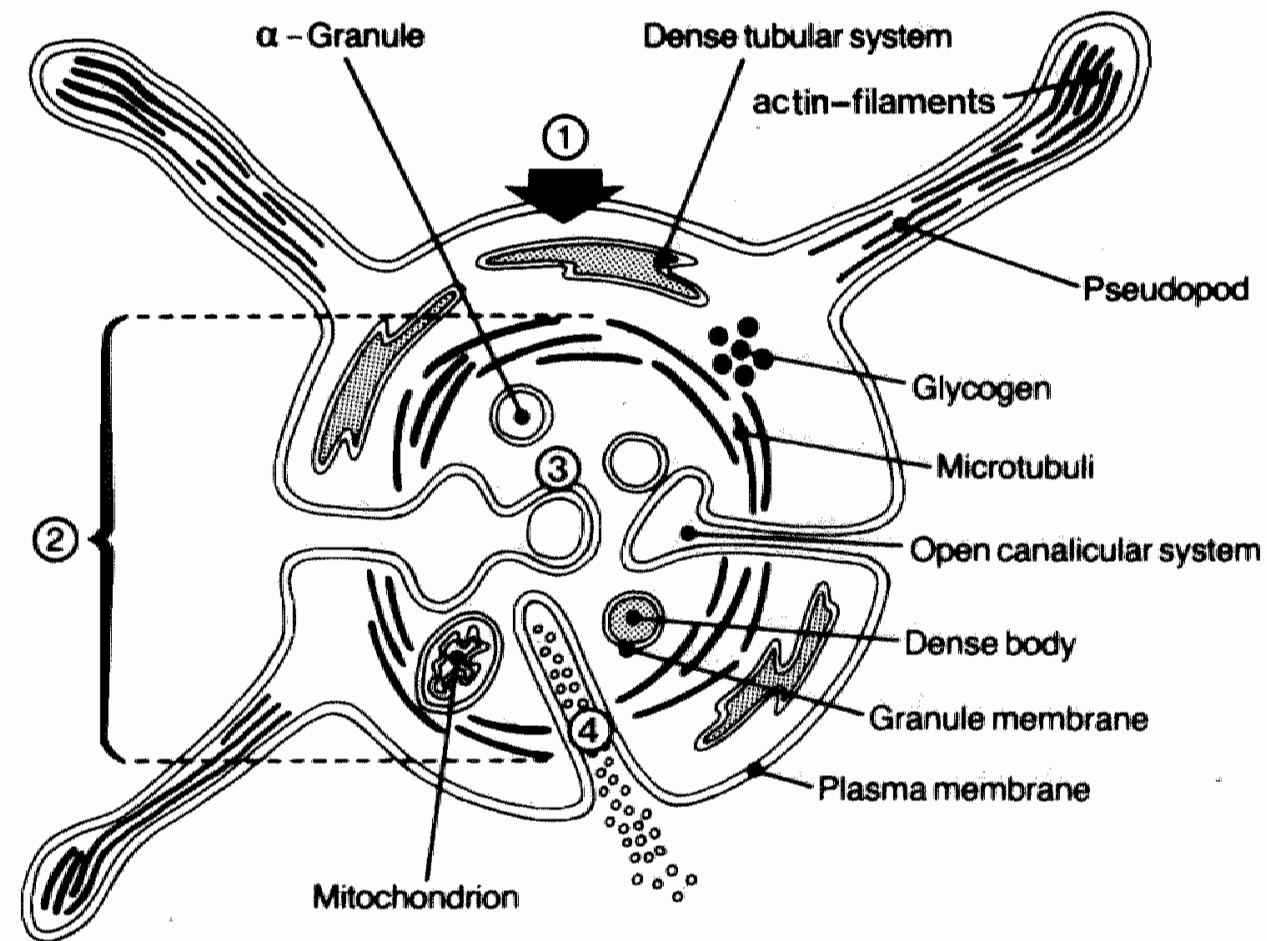

Fig. 2. Morphology of the stimulated platelet. Sehematic representation of structural features as observed by thin-section electron microscopy. Characteristic events of platelet stimulation are: agonist-receptor interacton (1), granule centralization (2), and fusion of granules with the open canalieular system (3) resulting in release of their contents (4). Further details are described in the text. 
pate in coagulation, like fibrinogen, factor $V$, and heparin-binding protein (platelet factor 4). Other proteins support adhesion of platelets to the subendothelium, like fibronectin, thrombospondin, and von Willebrand factor. a-Granules also contain $\beta$-thromboglobulin, albumin, several protease inhibitors, and platelet-derived growth factor, which stimulates the proliferation of arteriall smooth muscle cells and fibroblasts. The lysosomes hold a number of acid hydrolases, such as hyaluronidase, $\alpha-N$-acetylglucosaminidase and $\beta-$ glucuronidase $[38,290]$. Besides secretory vesicles, the platelet cytoplasm contains allso a small number of mitochondria and abundant glycogen granules.

Two membrane systems can be detected in platelets: the open canalicular system (OCS) andthe dense tubular system (DTS). The OCS forms many channel-like invaginations of the plasma membrane deep into the platelet interior. These channels serve to enlarge the surface area of the plasma membrane available for membrane fusion during the release reaction, and convey the substances released by the internalized granules to the surrounding fluid. The DTS is derived from the smooth endoplasmic reticulum of the parent megakaryocyte and forms a tubular network, not in contact with the OCS. The DTS is the major storage site for $\mathrm{Ca}^{2+}$, which is actively accumulated and which can be released into the cytoplasm during platelet activation [138]. At the surface of the DTS, formation of thromboxane $A_{2}$ from arachidonic acid takes place [357], and possibly also the de novo synthesis of fatty acids and phospholipids [96].

\section{Platelet responses and stimuli}

The initial reaction in hemostasis following injury to vascular endothelium is adhesion of platelets to subendothelial structures $[238,266$, $279,373,551,600,601]$ which comprise microfibrils of elastin, basement membrane-like amorphous material and collagen fibrils. of these structures, collagen is required to achieve full release and aggregation following adhesion. At high shear rates, von Willebrand factor (vWo is neccesary for adhesion. After binding of vWf to subendothellum, it can be recognized by a specific platelet receptor.
Most stimuli cause the platelet to change shape. This change involves the formation of very fine pseudopodia, followed by a gradual departure from the disk shape until it becomes a spiny sphere with much broader pseudopodia (Fig. 2) [440]. The formation of pseudopodia is dependent on a reorganization of the cytoskeleton, which is also responsible for the centralization of granules during shape change. Following granule centralization, platelets secrete the contents of their granula [602]. This release reaction involves discharge of granular contents into the open canalicular systems after fusion with the plasma membrane. The contents of $\alpha$ granules and dense-bodies are most easily released, while secretion from lysosomes is delayed and requires the more potent stimulatory agonists [290].

Aggregation of platelets requires extracellular $\mathrm{Ca}^{2+}$ and fibrinogen. When platelets are activated without stirring they do not aggregate but shape change and release still occur [727]. Aggregation results when dimeric fibrinogen binds to receptors on two different platelets $[167,279]$. Binding sites for fibrinogen are induced during platelet stimulation. Aggregation is reinforced by a lectin-like association between platelet-bound fibrinogen and thrombospondin, a large protein that is secreted from a-granules and remains attached to platelets $[371,439]$.

Activated platelets accelerate clot-formation in various coagullation assays $[53,399,676]$. They may trigger the intrinsic coagulation pathway by activating factor XII adsorbed on the surface of ADP-activated platelets [584]. Collagen-stimulated platelets may directly activate factor XI in the absence of factor $\mathrm{XII}_{\mathrm{a}}$ [676] Most powerful is the platelet procoagulant activity, which involves the exposure of negatively charged phospholipids at the platelet surface to provide a catalytic surface on which various coagulation factors interact [732].

In the final stage of hemostatic plug formation, the platelet/fibrin clot retracts [1.15]. This process is dependent the contractile activity of the platelet cytoskeletal apparatus, which is intercellulariy connected via transmembrane glycoproteins and fibrin.

Platelets can be stimulated by a variety of ligands, which can be understood in terms of temporal and spatial processes occurring after damage of the vessel wall. Initially, platelets will be activated in vivo by collagen and other 
fibrillar components of the subendothelial matrix, by ADP released from damaged cells, and by thrombin which is formed rapidly from circulating prothrombin at sites of vessel wall damage. These platelets secrete ADP, and will release cyclooxygenase products like endoperoxides and thromboxane $\mathrm{A}_{2}$ (TxA2) $[8,319$, $534,537]$. These compounds provide a positive feedback (autocoids), and, together with thrombin, activate other platelets not in direct contact with the subendothelial matrix, resulting in increased thrombus size. This positive feedback system is probably limited by haemodynamic factors which limit the effective concentration of activators at the periphery of the thrombus, and by stimulation of endothelial cells by thrombin and ADP to produce prostacyclin, which is a powerful inhibitory platelet agonist $\llbracket 246,247,667 \rrbracket$. Of the stimulatory agonists, thrombin, platelet-activating factor (PAF), and TxA2 are the most potent, promoting the full range of platelet responses [101, $169,263,534,667,727]$. Stimulation of platelets by collagen is dependent on secondary formation of $\mathrm{TxA}_{2}$, which may be related to the relatively small number of in direct contact with collagen $[8,447,557,680]$. ADP is able to provoke shape-change and a partial, reversible, aggregation (primary aggregation), but is dependent on $\mathrm{TxA}_{2}$ formation for expression of release and irreversible (secondary) aggregation [534]. Other stimulatory agonists are epinephrine [583], serotonin [169], and vasopressine [395a]. Epinephrine and serotonin are weak activators, dependent on TXA formation for induction of secretion and aggregation, and probably play a role by amplifying the responsiveness to other activators [94]. Vasopressin is able to induce all platelet responses. In vivo, any role for vasopressin and $\mathrm{PAF}$ in the activation of human platelets seems unlikely in view of their low abundance [532]. All platellet responses require adequate levels of metabolic ATP $[288,289,661,662]$.

\section{T The name cytoskeleton is used in an aspecific manner to describe any three-dimensional organization of cy- toskeletal proteins within a cell:}

\#All molecular weights indicated "refer to reduced proteins.

\section{Composition and function of the platelet cytoskeleton}

Cytoskeletal proteins constitute a major part $(20 \%)$ of total platellel proteins, and play important roles both in stimulatory processes, as well as in many, if not all, platelet responses. They have been extensively reviewed elsewhere. $[139,204,205,214,271,378,382,384,658,693$, $695,696,698]$. The building blocks are the thin filaments, polymers of actin. The organization of the cytoskeleton ${ }^{\dagger}$ is dependent on the regulation of actin polymerization and on the organizing effects of actin-binding proteins. Mechanical force is generated by controlled interaction between actin filaments and myosin filaments.

Monomers of actin ( $42 \mathrm{kDa}^{\#}$ ) can selfassociate in a noncovalent interaction to form actin filaments. The initial phase of polymerization is the formation of nuclei, which is greatly accelerated in the presence of cations, or can be induced by nucleating proteins that complex more than one actin monomer $[332,507]$. Actin filaments have a defined polarity, which is demonstrated by decoration with heavy meromyosin. The two ends of actin filaments are identified in this way as the barbed end and the pointed end. Longitudinal growth of actin filaments is favored at the barbed end. Polymerization of actin in platelets is regulated by profilin and gelsolin. Profilin (16 kDa) forms a 1: 1 complex with actin monomers, thus inhibiting the polymerization of actin [405]. In platelets monomeric actin is associated entirely with profilin [405]. Gelsolin (90 kDa) binds to the barbed end of actin filaments in the presence of micromolar free $\mathrm{Ca}^{2+}$, resulting in fragmentation of actin filaments $[82,383]$. Gelsolin remains attached to the barbed ends of the short filaments and acts as a capping protein.

Platelets are abundant in high-molecular weight actin-binding proteins, which orchestrate the functional changes in organization of the cytoskeleton during platelet activation [rewiewed in: 136, 332, 507, 615, 616]. Filamin (250 $\mathrm{kDa}$ ) and talin $(235 \mathrm{kDa})$ each comprise about $5 \%$ of totall platelet protein. Both proteins are elongated rods which form head-to-head dimers, and are involved in linking actin filaments to membrane proteins. Talin links bundles of actin filaments to the cytoplasmic domain of the fibronectin receptor in adhesion plaques 
[292]. In platelets, the glycoprotein (GP) $\mathrm{III}_{\mathrm{b}} \mathrm{III}_{\mathrm{a}}$ complex constitutes the fibronectin receptor $[203,226,486$ a]. Thus, talin may link actin filaments to $\mathrm{GP} \mathrm{H}_{\mathrm{b}} \mathrm{III}$ in platelets. Filamin is an actin-crosslinking protein [382], which links actin filaments to GP Ib $[207,472$, 608]. These properties suggest that filamin is responsible for the organization of a submembraneous cyloskeletal structure in platelets $[204,206]$. Platelet actinin $(100 \mathrm{kDa})$ exists as a heterodimer, and induces crosslinking and bundle formation of actin filaments in the $a b-$ sence of $\mathrm{Ca}^{2+}$, which is different from muscle $\alpha$-actinin [363]. In unstimulated platelets $a-a c-$ tinin is concentrated at the barbed end of actin filaments, adjacent to the plasma membrane [153]. In activated platelets, it is concentrated in pseudopodia [522]. There is evidence that at-actinin has an affinity for specific lipids, i.e. diacylglycerol $[84,85]$. Platelet spectrin $(240$ kDa) resembles brain spectrin (fodrin) instead of erythrocyte spectrin [215]. Just like in the erythrocyte, it has a peripheral location, and the proteins that could link spectrin to the plasma membrane (ankyrin and protein 4. 1) are also present in platelets [148, 204]. However, since the amounts of spectrin/ankyrin/protein 4. 1 in platelets are very small with respect to other actin-binding-proteins (filamin, talin, a-actinin), they cannot be important elements of a peripheral cytoskeletal structure in platelets.

The generation of mechanic force within platelets results from interaction of myosin filaments with actin filaments, which stimulate the $\mathrm{Mg}^{2+}$-ATPase activity of myosin [506]. Platelet myosin represents about $5 \%$ of total platelet proteins, and, like skeletal muscle myosin, consists of two heavy chains of $200 \mathrm{kDa}_{\mathrm{s}}$ two light chains of $20 \mathrm{kDa}$ and two light chains of 16 $\mathrm{kDa}[3]$. The two heavy chains form a rod with a globular head to which the light chains are attached. Platelets may contain two different pools of myosin: one cytosolic and one membrane bound [430, 492]. Bipolar filaments of myosin have not been observed in intact platelets, but can be induced in solubilized platelets [454]. Polymerization of myosin is enhanced when myosin light chain (MLC) kinase phosphorylates the $20 \mathrm{kDa}$ light chains of myosin, or when the concentration of $\mathrm{Mg}^{2+}$ ATP is reduced [576]. In intact platelets, where high concentrations of $\mathrm{Mg}^{2+}-\mathrm{ATP}$ are present, phosphorylation/dephosphorylation is the major mechanism to regulate the interaction between myosin and actin filaments. Other factors which regulate the interaction between myosin and actin filaments are tropomyosin, troponin, and caldesmon [116, 615]. Platelet tropomyosin ( $31 \mathrm{kDa}$ ) forms a heterodimer [132], and is believed to prevent the binding of myosin to actin filaments, like skeletal muscle tropomyosin, in defined regions of the cell [204, $489,604]$. Troponin thas been isolated from pig platelets, and shown to participate in the modulation of actin-activated myosin-ATPase [639]. Caldesmon $(80 \mathrm{kDa})$ binds to actin filaments in the presence of $\mathrm{Ca}^{2}+$, and binds to calmodulin in the absence of $\mathrm{Ca}^{2+}$ [520]. Thus, caldesmon has been suggested to regulate the binding of myosin to actin filaments. Caldesmon is also found in platelets, showing a similar $\mathrm{Ca}^{2+}$-dependent behavior as smooth muscle caldesmon [165].

Platelet cytoskeleton research nowadays discriminates between cytoplasmic skeleton and membrane skeleton $[29,206,207,210,446$, $\left.471_{*} 482,546\right]$. These terms can be defined both operationally and morphologically. The cytoplasmic skeleton refers to the precipitate obtained after low-speed centrifugation of cells solubilized with $1 \%$ Triton $\mathrm{X}-100$. The remaining soluble fraction contains also cytoskeletal proteins, which can be isolated by high-speed centrifugation. In addition, the high-speed fraction contains many of the plasma membrane glycoproteins, which are complexed to actin filaments by actin-binding proteins [206]. Therefore the high-speed fraction is taken to represent a special structure, the membrane skeleton, a network of short actin filaments lining the plasma membrane and coupled to transmembrane proteins via actin-binding proteins. Most likely, the cytoplasmic and membrane skeleton only reflect structures which remain stable in Triton X-100 and which have lost part of the associated proteins. From a morphological point of view, both thin section $[204,210]$ and quick-freeze deep-etch electron microscopy $[446,471]$ clearly reveals the existence of a cytoskeletal structure lining the cytosolic face of the platelet plasma membrane, supporting the membrane skeleton as a functional concept.

In unstimulated discoid platelets, 40 to $50 \%$ of the total platelet actin is in a filamentous form $[211,301]$. Free actin monomers in platelets are associated with profilin [405]. Profilin is not a very effective inhibitor of actin polymerization once polymerization nuclei 
(like the barbed ends of actin filaments) are present [332]: Thus, it appears that profilin acts more as a buffer, and that nuclei are capped. Several candidates for barbed-end capping proteins exist in platelets. One of them is gelsolin [382]. Other candidates are proteins that link actin filaments to the plasma membrane, because polymerization appears to occur in a submembranous location and because the barbed ends of actin filaments in activated platelets are adjacent to the plasma membrane [440]. Talin is such a link-protein that has been found to cap actin filaments in purified systems [123], a-Actinin is another candidate for a capping proteun $[332,616]$. The cytoplasmic skeleton of unstimulated platelets contains, besides actin filaments, the actin-binding proteins: filamin, a-actinin, and tropomyosin [239, 301, $541,546,717,718]$. However, most of the cytoskeletal proteins in unstimulated platelets are
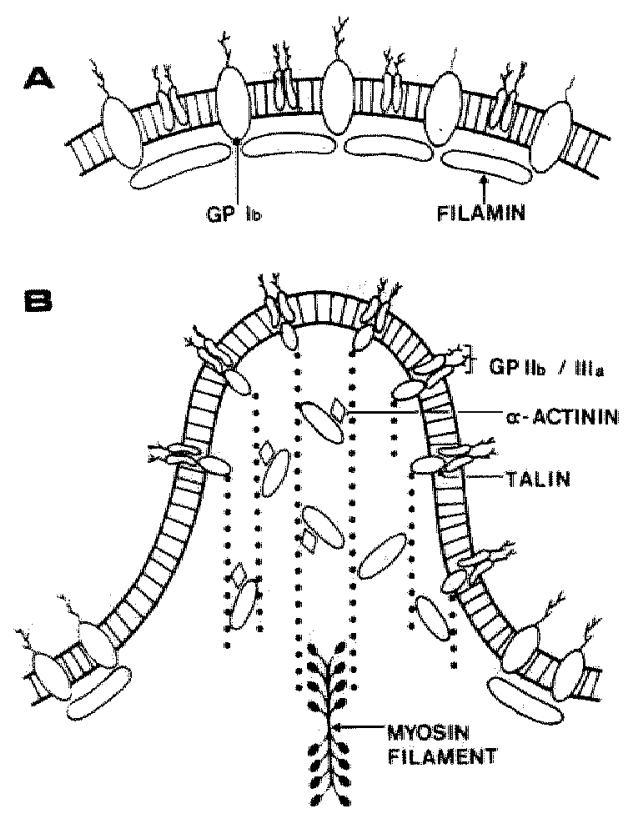

Fig. 3. Tentative model for the involvement of the cytoskeleton in the formation of pseudopodia during platelet activation. In unstimulated platelets. (A) Giamin is associated with GP Ib. Upon stimulation of platelets, phosphorylation of filamin and polymerization of actin results in the outgrowth of a bundle of actin to which GP Ib/ $\mathrm{II}_{\mathrm{a}}$ becomes crosslinked through a linkage protein which may be tallin, in view of its involvement in cell-cell contact [see text]. Interaction of the actin bundles with myosin (bottom) provides the force for clot-contraction. Adapted from [231]. present in the membrane skeletton, which contains, beside short actin filaments, several actin-binding proteins (filamin, talin, myosin, and a-actinin), and several membrane glyooproteins (GP Ib, GP IX, and as minor components: GP $I_{a}$ GP II $I_{a}$, and GP 250) [206, 207, 253]. Through its linkage to GP Ib/XX complex the membrane skeleton determines the organization of this complex within the membrane [210], and regulates the ability of GP Ib to bind to $v W f[119]$. Another component of the platelet cytoskelleton are the microtubules [139, $698]$. Tubulin $(55 \mathrm{kDa})$ is a major platelet protein, and is polymerized into a single coil that is wound tightly on itself at the periphery of the platelet underneath the membrane skeleton. The peripheral microtubule coil lies in the equatorial plane and is involved in the maintenance of the discoid shape of the platelet.

Stimulation of platelets results in an increase in actin polymerization, comprising 70 $80 \%$ of the total actin $[90,301]$. The critical step in inducing actin polymerization within platelets may be the release of a barbed-end capping protein, or the formation of new nuclei [204]. In addition, dissociation of actin from profilin may be an important step, which has been related to the metabolism of polyphosphoinositides $[369,370]$. During pllatelet stimullation the organization of actin filaments dramatically changes from loose networks into bundles of actin filaments in pseudopodia, and rings of actin filaments surrounding centralized granules $[68,214,309,379,380,388,480,693$, 695,6961 . Simultaneously, changes in the distribution of filamin, a-actinin,and myosin take place, which is evidenced by the increase of these proteins in the cytoplasmic skeleton, and a concomitant decrease of the same proteins in the membrane skeleton [206, 207]. Myosin becomes phosphorylated and as a result associates with actin filaments [213], thus generating the tension required for centralization of granules. Centralization of granules is related to, but not dependent on, centripetal reorganization of the microtubule coil [698]. The contractile force for granule centralization is generated by actomyosin. In addition, elongated actin filaments bundle through the action of filamin and $\alpha$-actinin, and support the outgrowth of pseudopodia [440]. Phosphorylation of filamin may provide a mechanism for modulating its affinity for actin [724]. The glycoprotein IIb/II ${ }_{\text {a }}$ complex in the plasma mem- 
brane becomes linked to the actin filament bundles $[481,688]$ (Fig. 3) in the pseudopodia, thereby permitting, via its binding to fibrinogen, tension to be transferred from the cytoskeleton to the extracellular fibrin network.

\section{Structure and function of the platelet plasma membrane}

The platelet plasma membrane is the best studied and probably most complex organelle of the platelet. As a general feature of biological membranes it is composed of a lipid bilayer which contains both transmembrane as well as peripheral proteins. It functions as a permeability barrier through which transport of solutes and ions is carefully controlled by transport proteins and ion-channels. It is the locus of receptor proteins by which a variety of ligands may trigger or inhibit platelet activation. In addition, it contains many proteins involved in signal transduction by generation of second messengers. The plasma membrane is covered at its cytosolic side by a network of cytoskeletal proteins, which may modulate the properties and organization of membrane proteins, and which contributes to its elastic properties. Moreover, the platelet plasma membrane plays an intricate role in the expression of platelet responses: during shape-change it is involved in pseudopod formation, during the release reaction granules fuse with the plasma membrane, and the formation of a procoagulant platelet surface is dependent on changes in lipid distribution. The components of the platelet plasma membrane and their function has been reviewed elsewhere $[46,110,138,350,467,498,607,730$, 731].

The lipid bilayer contains $70 \%$ phospholipids by weight, while the remainder predominantly consists of cholesterol plus a small amount of glycolipids. The cholesterol content of the plasma membrane is two to three times higher than that of intracellular membranes $[186,243,358,418]$. Five major phospholipid classes have been identified in human platelets: phosphatidylcholine (PC), phosphatidylethanolamine (PE), sphingomyelin (SphM), phosphatidylserine (PS), and phosphatidylinositol (PI) $[79,96,98,195,243,358,396,418,494$, 569]. PE consists for about $70 \%$ of plasma-
logen-PE [79]. The phospholipids are asymmetrically distributed over the bilayer $[96,98$, 494,5681 . The outer leaflet of the plasma membrane contains almost all SphM and is enriched in PC, while the cytosolic leaflet contains most, if not all, of the PS and PI and is enriched in PE. For further details about phospholipid transbilayer asymmetry is referred to the section concerning platelet procoagulant activity. Not only the phospholipids but also their acyl chains are asymmetrically distributed over the plasma membrane. The major part of esterified arachidonic acid in the plasma membrane is present in the cytosolic leaflet [494], where it is incorporated in PC, PE and PI at the sn2 position. Though PE and PI are enriched in arachidonic acid to a higher degree than PC, most of the arachidonic acid is present in $\mathrm{PC}$ due to its high abundancy $[396,358]$.

The plasma membrane has a unique enzymatic composition different from intracellular membranes (DTS), as is revealed by separation of platelet membranes by gradient centrifugation and free flow electrophoresis [138]. Adenylate cyclase is probably exclusively located in the plasma membrane. Phospholipase $\mathrm{A}_{2}$, diglyceride lipase, cyclooxygenase, and thromboxane synthase, the machinery for production of $\mathrm{TxA}_{2}$, is predominantly located in intracellular membranes (DTS). The distribution of lipoxygenase is less extreme. $\mathrm{Ca}^{2+} / \mathrm{Mg}^{2+}$-ATPase activity seems only present in intracellular membranes, suggesting that extrusion of cytosolic $\mathrm{Ca}^{2+}$ via the plasma membrane does not play a significant rolle in intracellular $\mathrm{Ca}^{2+}$ homeostasis.

The plasma membrane is coated on the exterior with an electron dense glycocalyx, representing the carbohydrate rich domains of membrane glycoproteins. These glycoproteins function as receptors for plasma-borne stimuli, and play a role in the adhesion of platelets to the subendothelium and in platelet aggregation. Their receptor function and their absence as a cause of many congenital platelet disorders has lead to extensive investigations with respect to isolation and characterization of platelet membrane glycoproteins [reviewed in: $41,43,46$, $108,109,110,117,120,230,350,391,466,467$, $468,469,495,497,498,500,608]$.

Glycoprotein $I_{a}(167 \mathrm{kDa})$ is a relatively minor platelet surface component, which contains several intrachain disulphide bonds [110]. There is evidence that GP $\mathrm{I}_{a}$ is linked to the 
platelet oytoskeleton via filamin [212]. Findings with a patient defective in GP Ia [455] suggest that GP I functions as collagen receptor.

Glycoprotein $\mathrm{I}_{b}$ is the major sialoglycoprotein, consisting of an a-chain (150 kDa) and a $\beta$-chain $(26 \mathrm{kDa})$, which both are transmembrane proteins linked together by disulphide bonds [496]. The extracellular part of the achain is easily hydrolysed by various proteases, resulting in the release of a large water-soluble fragment, termed glycocalicin [268, 473, 653]. The cytoplasmic segment of the $\beta$-chain can be phosphorylated $[67,609,711]$. In situ, GP $\mathrm{I}_{\mathrm{b}}$ is present in a 1: 1 complex with GP IX (22 kDa) $[42,44,121,174,209,419,700]$. In unstimulated platelets $G P I_{b}$ is linked to the membrane skeleton via filamin $[206,207,472,608,609]$. GP Ib contains a binding site for thrombin [225, $268,412,631]$. In addition, GP $I_{b}$ is involved in adhesion of platelets to the subendothelium as a binding site for vWf adsorbed to collagen [120].

Glycoprotein $\mathrm{II}_{\mathrm{a}}(150 \mathrm{kDa})$ is a less studied protein. It contains several intramolecular disulfide bonds, and it may be complexed to GP Ia $[149,353,562]$. The function of $\mathrm{GP} \mathrm{II}_{\mathrm{a}}$ is unknown, but there are indications that it is structural similar to a family of proteins called "very late activation" antigens [502].

Glycoproteins $\mathrm{II}_{b}$ and $\mathrm{III}_{\mathrm{a}}$ are major components of the platelet plasma membrane where they are distributed as heterodimer complexes, which play an important role in platelet aggregation and reinforcement of adhesion [reviews:" 117, 498]. GP IIb/IIIa exist also as an intracellular pool in the membrane of a-granules $[686,709]$. GP II $b$ is composed of two disulfide-linked subunits, $(132 \mathrm{kDa})$ and $\beta, 25$ $\mathrm{kDa})$. GP III ( $110 \mathrm{kDa})$ is a single polypeptide chain crosslinked by multiple intrachain disulfides [175]. Formation of the GP $\mathrm{II}_{\mathrm{b}} / \mathrm{III}_{\mathrm{a}}$ complex is dependent on $\mathrm{Ca}^{2+}$, since chelation of $\mathrm{Ca}^{2+}$ dissociates the complex $[202,221,592]$. Morphological investigations [91] showed that the complex consisted of a globular head (GP IIb) and a rod-like tail (GP III $\left.{ }_{a}\right)$. In unstimulated platelets the GP $\Pi_{b} / \Pi_{a}$ complex is evenly distributed in the plasma membrane [686]. Activation of platelets results in clustering of the complexes [19, 387], and increased binding to the cytoskeleton $[501,689]$. The major function of the GP $\mathrm{II}_{\mathrm{b}} / \mathrm{TI}_{\mathrm{a}}$ complex is to form a binding site for adhesive proteins, like fibrinogen [ 403 , $491]$, fibronectin $[203,226,486 \mathrm{a}]$ and $\mathrm{vWf}[400$,
488]. Binding these proteins and linking them to the actinomyosin of the platelet cytoskeleton provides the mechanism for clot retraction. A common recognition mechanism has been proposed for these adhesive proteins: they all contain the sequence Arg-Gly-Asp, which is recognized by GP $\mathrm{II}_{\mathrm{b}} / \mathrm{II}_{\mathrm{a}}[226,278,503,523]$. In addition to the tripeptide sequence, GP $\mathbf{M b} / \mathrm{III}_{\mathrm{a}}$ may have a second class of binding sites for adhesive proteins $[326,362,561]$. The binding site for fibrinogen on GP IIb/MI $a$ is dependent on intimate association of both glycoproteins [310], and hence on the presence of $\mathrm{Ca}^{2+}$. In unstimulated platelets the binding site for fibrinogen is not expressed. Its expression requires conformational changes during platelet activation $[118,120 \mathrm{a}, 594]$. The regulation of the expression of the fibrinogen binding site on GP $\mathrm{II}_{\mathrm{b}} \mathrm{III}_{\mathrm{a}}$ is still unclear, though it has been suggested to be dependent on phospholipase $\mathrm{C}$ activation [591], and on interaction of GP IIb/III with the cytoskeleton [35, 491]. Binding of vWf, secreted by platelets, to $\mathrm{GP} \mathrm{II}_{\mathrm{b}} / \mathrm{MI}_{\mathrm{a}}$ on activated platelets may be unwolved in the reinforcement of platelet aggregation [488]. In addition to its role in platelet aggregation, some findings suggest that the GP $\mathbb{I I}_{b} / \mathbb{I I}_{a}$ complex may be involved in uptake of $\mathrm{Ca}^{2+}$ by platelets. The complex contains the major part of $\mathrm{Ca}^{2+}$ on the platelet surface $[73,77,221,302,592]$, and $\mathrm{Ca}^{2+}$ uptake by platelets is blocked by some antibodies to GP II $/ \mathrm{III}_{\mathrm{a}}[65,518,712]$.

Glycoprotein IV $(90 \mathrm{kDa})$, also known as $\mathrm{III}_{\mathrm{b}}$, is a major protein which is poorly understood. It is a single chain polypeptide, and it is very hydrophobic $[635,654]$. Glycoprotein IV may function as a receptor for thrombospondin [18]"

A minor platelet glycoprotein which has received much attention is GPV $(82 \mathrm{kDa})$ [45]. This protein can be hydrolyzed by thrombin $[328,329,691]$ and other proteases $[46,411$, $701]$ in a water soluble fragment $(69 \mathrm{kDa})$ and a hydrophobic fragment $(20 \mathrm{kDa})$, left behind in the membrane.

In view of the importance of collagen and thrombin in the physiological stimulation of platelet procoagulant activity, special attention will be given to their mode of action at the surface of the platelet plasma membrane. To initiate platelet aggregation, collagen requires not only its native conformation, a triple helix composed of three polypeptide chains , but also a fibrillar structure [560]. The repeating units dis- 
played by collagen fibrils allow for multimeric interactions with collagen receptors, interactions required for optimal platellet response to collagen. Interaction of platelets with collagen can be divided in attachment and spreading. Attachment may involve accessory proteins, which bind both to platelets and collagen, like fibronectin, thrombospondin, and wWF. Studies of a patient with a haemorrhagic disorder, whose platelets lacked GP Ia and were unresponsive to collagen, clearly implicate $\mathrm{GP} \mathrm{I}_{\text {a }}$ as collagen receptor $[455,456]$. These implications have recently been extended by observations that a complex of $\mathrm{GP} \mathrm{I}_{\mathrm{a}}$ and $\mathrm{GP} \mathrm{II}_{\mathrm{a}}$ mediates adhesion of platelets to collagen $[353,562]$, and by a study of another patient with decreased adhesion to collagen [314]. The situation is complicated, however, by suggestions that GP $\Pi_{b}$ is involved in platelet adhesion to collagen $[335,590,657]$. In addition, specific binding of a $61 \mathrm{kDa}$ [334], a $65 \mathrm{kDa}$ [100], a 80 $\mathrm{kDa}$ [359], and a $160 \mathrm{kDa}$ [559] membrane protein to collagen has been described. To reconcile these findings, it seems most likely that platelet stimulation by collagen inwolves several different binding sites [589]. The interaction of collagen with platelets is reviewed elsewhere $[26,99,360,495,560]$.

The interaction of thrombin with the platelet plasma membrane is subject to considerable controversy. Despite numerous studies, it is still not clear whether thrombin stimulates platelets by receptor interaction, or by a proteolytic event, or both. Mary observations suggest that proteolytic activity of thrombin is required for stimulation of platelets. Inhibition of its proteolytic activity makes thrombin ineffective as platelet stimulator [368], while other proteases, like trypsin, a-clostripain, papain, and thrombocytin, are also able to activate platelets [46]. The best known substrate for thrombin in platelet membranes is GP V $[329,691]$. However, despite initial suggestions that GP V hydrolysis may be required for stimulation of platelets by thrombin [410], recent observations clearly suggest that hydrolysis of GP V is not involved in platelet activation by thrombin $[56$, $300,409,691]$. In fawor for the importance of thrombin receptor interaction are recent observations that small peptides which do not affect the proteolytic activity of thrombin have antagonistic activity [547]. Platelets contain three classes of binding sites for thrombin: a high-affinity, a moderate affinity, and a low affinity class [270], which recognize different regions of thrombin. The high affinity binding site represents the functional thrombin receptor on platelets [269], though the moderate affinity binding site may take over this function [267]. Early suggestions that GP Ib constitutes a binding site for thrombin, have been confirmed by the effect of antibodies $[412,714]$, by crosslinking experiments [631], and by direct binding assays $[225,268]$. It has been suggested that both the active-site and the fibrinogen binding site of thrombin are involved in binding to GP $\mathrm{Ib}_{\mathrm{b}}$ [260]. However, platelets which lack GP' Ib after treatment with selective proteases, still respond to thrombin, albeit retarded [410]. This could be explained by the moderate affinity binding site, which is still present after limited protease treatment, and which may function as thrombin receptor in absence of the high-affinity binding site [267]. In addition, platelet activation by thrombin is not affected by the presence of excess active-site inhibited thrombin, which shows identical binding characteristics as thrombin $[46,328]$, though inhibition of the proteolytic activity of thrombin can influence its binding to GP Ib $[260,269]$. The matter becomes most confusing when Bernard-Soulier platelets are considered. These platelets lack both GP Ib and GP V, yet they respond to high doses of thrombin. To account for these diverse findings, complicated models have been proposed [46], which include proteolysis of the thrombin receptor itself, not necessarily resulting in the loss of a fragment. In addition, it may well be that there is more than one functional for thrombin [409]. Interaction of thrombin with platelets has been discussed in several reviews $[43,46,160,199,495]$.

\section{Platelet signal transduction}

Despite a variety of platelet stimuli (vide supra), platelets display a characteristic sequence of responses upon stimulation. The past ten years ample evidence has been provided that the molecular pathways involved in transduction of the stimulatory signal to the expression of platelet responses are only few in number, and are used by many platelet agonists [reviews: $8,169,198,231,232,263,272,289,319$, $357,534,537,581,582,395 \mathrm{a}, 605,646]$. A cen- 
tral role in platelet activation is played by cytosolic $\mathrm{Ca}^{2+}\left(\left[\mathrm{Ca}^{2+}\right] \mathrm{i}\right)$. This is suggested by observations that $\mathrm{Ca}^{2+}$ ionophores are able to elicit the same cellular and molecular responses as physiological agonists do with the exception of stimulation of phospholipase C $[198,538]$. The use of permeabilised platelets $[581,582]$ further demonstrated the importance of $\left[\mathrm{Ca}^{2+} \mathrm{b}\right.$ and the effects of mucleotides and protein phosphorylation on the $\mathrm{Ca}^{2+}$ requirements for platelet activation. In addition, the use of intracellular fluorescent (chlortetracycline, Quin2, Fura2) and luminescent (aequorin) calcium indicators provided detailed information about $\left[\mathrm{Ca}^{2+}\right]_{i}$ in terms of sources, levels, and regulation $[191,263,264,265,303$, $434,508,510,525,532,534,535,550,641,680$, $681]$. Resting $\left[\mathrm{Ca}^{2+}\right]$ in platelets is around 80 $100 \mathrm{nM}[508,521,535]$. During platelet activation $\left[\mathrm{Ca}^{2+}\right.$ ] will rise to $1-5 \mathrm{mM}$ due to mobilization of intracellular $\mathrm{Ca}^{2+}$ and influx of extracellulair $\mathrm{Ca}^{2+}[169,263]$. In addition to $\left[\mathrm{Ca}^{2+}\right]_{\mathrm{i}}$ and in synergistic cooperation, protein kinase $\mathrm{C}$ is clearly involved in platelet signal transduction $[231,305,462]$. More recently, guanine nucleotide-binding regulatory proteins (G-proteins) have entered the scheme as a direct link between receptors and phospholipases and ion-channels $[81,272,386,452]$. Though understanding of the mechanisms of signal transduction in platelets has gained much, in- volvement of as yet unidentified mechanisms may operate in platelets activated by ADP [534]. Platelet activation is complicated by the actions of autocoids, especially $\operatorname{TxA} 2[8,357]$, formed during platelet stimulation and functioning as a positive feedback. Finally, inhibition of platelet activation, via the production of cAMP, is provided by prostacyclin, resulting from the stimulation of endothelial cells by ADP and thrombin, and partly dependent on platelet derived endoperoxides [357, 646].

It has become evident that one of the first actions of stimulatory agonists on platelets is a rapid degradation of polyphosphoinositides (phosphatidylinositol-4-phosphate (PIP), and phosphatidylinositol-4,5-bisphosphate (PIP2) by polyphosphoinositide-specific phospholipase $\mathrm{C}$ (a phosphodiesterase), resulting in the formation of inositol-1,4,5-trisphosphate (IP3) and 1,2-diacylglycerol (DAG) $[145,169,272$, 706]. The degradation and resynthesis of phosphoinositides (PI-cycle) has evolwed into a complex set of reactions (Fig. 4). The DAG formed is enriched with stearate and arachidonate, and for a large part rapidly phosphorylated to stearoylarachidonyl phosphatidic acid (PA) [407], often used as an indicator for phospholipase $C$ activity. In thrombin-activated platelets, the cellular content of PIP2 is rapidly decreased followed by a compensatory resynthesis above resting values, while the content of PIP' is

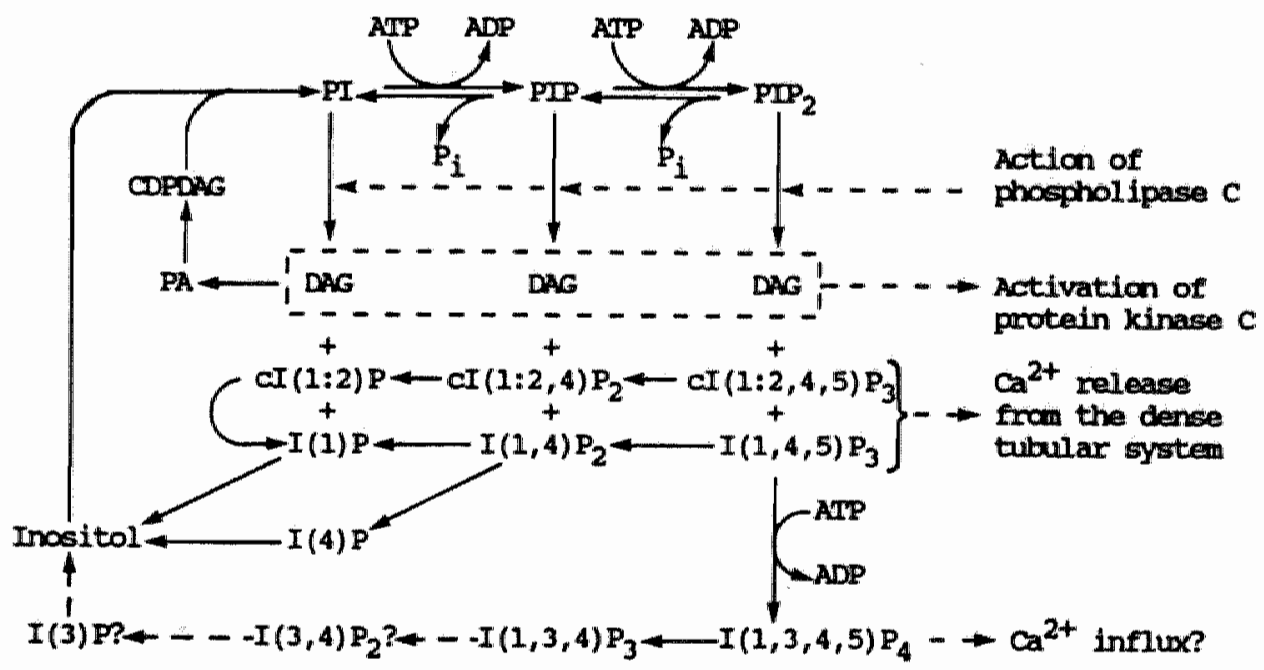

Fig. 4. Metabolism of phosphoinositides and inositol phosphates in platelets, and the importance of some of its components in platelet activation. Abbreviations are explained throughout the text. All inositols and inositides have the D-myo-inositol configuration. Figure is taken from [272]. 
not altered. Resynthesis of polyphosphoinositides results in a drop in the content of PL. The action of phospholipase $C$ results in the generation of various inositol 1: 2-cyclic phosphates [297, 638] and inositol 1-phosphares. Best known is $\mathrm{IP}_{3}$, which is dephosphorylated back to inositol ${ }_{i}$ or phosphorylated to I $(1,3,4,5) \mathrm{P}_{4}$ (IP4) and even higher phosphates. cI(1: $2,4,5) \mathrm{P}_{3}$ is hydrolysed much more slowly than $\mathrm{IP}_{3}$ and was found to release $\mathrm{Ca}^{2+}$ from permeabilized platelets with a similar potency as IP $\mathbf{P}_{3}[705]$. It has become clear that the stimulation of phospholipase $\mathrm{C}$ by agonist-receptor complexes is mediated by $G$-proteins $[272,366$, 367]. G-proteins are membrane-bound heterotrimers, consisting of an $\alpha, \beta$, and $\gamma$-subunit, of which the $\alpha$-subunit contains the GTP bindingsite. G-proteins become active upon receptor occupation by exchanging bound GDP for GTP, and are inactivated by an intrinsic GTPase activity. The $\mathrm{G}$-protein that stimulates phospholipase $\mathrm{C}$ is different from those which stimulate $\left(G_{\mathbb{S}}\right)$ or inhibit $\left(G_{i}\right)$ adenylate cyclase, and is referred to as $G_{p}$ [111]. Phospholipase $C$ can be inhibited by cyclic AMP (cAMP), most likely by uncreasing the GTPase activity of $G_{p}$ [272]. The second messengers generated by phospholipase $\mathrm{C}$ are $\mathrm{DAG}, \mathrm{IP}_{3}$, and probably IP4. DAG will stimulate protein kinase C (Ckinase) [460, 461], $\mathrm{IP}_{3}$ is able to promote $\mathrm{Ca}^{2+}$ release from the DTS $[6,75,169,478,527,588]$, and $\mathrm{IP}_{4}$ is suggested to be involved in influx of extracelluilar $\mathrm{Ca}^{2+}[293]$.

C-kinase consists of a family of enzymes that transfer phosphate from ATP to serine or threonine residues of specific proteins in the presence of $\mathrm{PS}$ and $\mathrm{Ca}^{2+}$ [459]. DAG stimulates $\mathrm{C}$-kinase by lowering its $\mathrm{Ca}^{2+}$ requirement to submicromolar levels. C-kinase can be stimulatted by some phorbol esters and synthetic diacylglycerols. An alternative way to stimulate C-kinase is suggested by observations that limited proteolysis by calpain renders $\mathrm{C}$ kinase active independent of PS, DAG and $\mathrm{Ca}^{2+}[323,636]$. An important aspect in stimulation of $\mathrm{C}$-kinase is its translocation from the cytosol in resting platelets to the membrane in response to cell stimulation. Mechanisms governing the translocation of $\mathrm{C}-\mathrm{kinase}$ are poorly understood, and may involve increased $\mathrm{Ca}^{2+}$ concentrations. C-kinase can modulate both stimulatory and inhibitory pathways of platelet activation, and is thus suggested to be a bidirectional cell modulator [169]. The stimulatory ac- tions of $\mathrm{C}$-kinase operate in harmony with the actions of $\mathrm{Ca}^{2+}$ to produce synergistic responses $[462,632]$. The major substrate of $C$-kinase in platelets is a $47 \mathrm{kDa}$ protein (P47). $\mathrm{P} 47$ is a solubile protein, and its phosphorylation is extensively correlated with the release reaction, more specifically with granule fusion [231]. The identity of P47 is not know, though several bypothesis have been advanced [272]. Phosphorylation of filamin by $\mathrm{C}-$ kinase during platelet activation has been postulated to regulate the cytoskeletal reorganization involved in the formation of pseudopodia [231]. An other group of important substrates of $\mathrm{C}$-kinase are the $\alpha-$ subunits of various, if not all, $G$-proteins [81, 169 , $272,386,452]$. By phosphorylation of $\mathrm{G}_{\mathrm{r}}-\alpha, \mathrm{C}-$ kinase supresses the stimulation of adenylate cyclase. Inhibition of phospholipase $C$, evidenced by the effects of phorbol ester on PIP2 hydrolysis and mobilization of intracellular $\mathrm{Ca}^{2+}$, is most likely due to phosphorylation of $\mathrm{G}_{\mathrm{p}} \alpha$. Inhibition of influx of extracellular $\mathrm{Ca}^{2+}$ through ion-channels by phorboll ester [504, 505] can also be explained by the action of $C_{-}$kinase towards G-proteins. Phosphorylation of MLC by C-kinase constitutes another inhibitory action, resulting in suppression of actin-activated ATPase activity of myosin which has been phosphorylated by MLC kinase [231].

Cytosolic $\mathrm{Ca}^{2+}$ increases during platelet activation as a result of mobilisation of $\mathrm{Ca}^{2+}$ rrom the DTS by $\mathrm{IP}_{3}$, and influx of extracellular $\mathrm{Ca}^{2+}$ through receptor-operated divalent ionchannels (ROC's) $[169,263,272,533,581,646]$. In case of threshold agonist concentrations, $\mathrm{Ca}^{2+}$ mobilization is dependent on cytosolic alkalization by the $\mathrm{Na}^{+} / \mathrm{H}^{+}$exchanger [597]. Important pathways by which increased $\left[\mathrm{Ca}^{2+}\right]$ exerts its intracellular effects are: $\mathrm{Ca}^{2+} / \mathrm{cal}-$ modulin-dependent processes, $\mathrm{Ca}^{2+}$-dependent neutral cysteine protease (calpain), and phospholipase $\mathrm{A}_{2}$. In addition, $\mathrm{Ca}^{2+}$ directly affects the cytoskeletal organization by modulation of the binding properties of cytoskeletal proteins and by microtubule depolymerization $[316,427]$, and $\mathrm{Ca}^{2+}$ facilitates fusion between granule membranes and the plasma membrane [385]. Calmodulin can bind up to $4 \mathrm{Ca}^{2+}$ ions, leading to substantial conformational changes. The $\mathrm{Ca}^{2+} / \mathrm{CaM}$ complex modulates the activity MLC kinase [231], and phosphorylase b kinase [646] which regulates glycogenolysis. In addition, CaM has been shown in other cells to be 
involved in $\mathrm{Ca}^{2+}$-dependent regulation of $\mathrm{cy}$ clic nucleotide phosphodiesterase, $\mathrm{Ca}^{2+}$-dependent protein phosphatase (calcineurin), adenylate cyclase, and the calcium pump $\left(\mathrm{Ca}^{2+} / \mathrm{Mg}^{2+}\right.$-ATPase $)[70,135,178,646]$. In platelets, adenylate cyclase and cyclic nucleotide phosphodiesterase may not be regulated by CaM [692]. Regulation of cytoskeletal organization by $\mathrm{Ca}^{2+} / \mathrm{CaM}$ is thought to proceed via a so-called "flipflop" mechanism [465], i.e. caldesmon binds to actin at low $\left[\mathrm{Ca}^{2+}\right]_{i}$, but binds to $\mathrm{CaM}$ at elevated $\left[\mathrm{Ca}^{2+}\right]$. This switch in binding of caldesmon is believed to act as an on-off switch for actin-myosin interaction. To which extent $\mathrm{CaM}$ exerts the same effects in platelets is not clear [646]. Limited proteolysis by calpain may be an underestimated mediator of the $\mathrm{Ca}^{2+}$ signal. Among its substrates are many cytoskeletal and membrane-bound proteins. Of particular interest for platelet signall transduction are the findings that $\mathrm{C}$-kinase $[323,636]$, phospholipase C [390], MLC kinase $[306,307,675]$, and adlenylate cyclase $[4,647]$ can be irreversibly activated by calpain. In view of its importance in the context of this thesis, calpain will be discussed in more detail elsewhere (vide infra).

Of special importance in mediating the $\mathrm{Ca}^{2+}$ signal in platelets is the hydrolysis of phospholipids by phospholipase A2 $[272,537]$. Evidence is increasing that phospholipase $\mathrm{A}_{2}$ exists in multiple forms with different $\mathrm{Ca}^{2+}$ requirements [22]. This can explain the paradoxical findings that platelet phospholipase $\mathrm{A}_{2}$ can be stimulated by increased $\left[\mathrm{Ca}^{2+}\right]_{\mathrm{i}}[97,337$, $508]$, but also independent of $\left[\mathrm{Ca}^{2+}\right]_{i}$ by receptor occupation $[83,272,508]$. Part of phospholipase $A_{2}$ is bound to the plasma membrane and the DTS [138], while the majority is soluble [337]. It seems most likely that phospholipase $A_{2}$ bound to the plasma membrane is activated directly by a $\mathrm{G}$-protein after agonist-receptor binding $[83,223,443,444,445]$, and that a soluble phospholipase $\mathrm{A}_{2}$ becomes bound to membranes only after $\mathrm{Ca} 2+$ mobilization. The important product generated by phospholipase $\mathrm{A}_{2}$ is arachidonic acid, which is derived from PE, PC, and PI [78, 605]. Arachidonic acid can also be formed from $D A G$, produced by phospholipase $\mathrm{C}$, vila the action of a di- and monoacylglycerol lipase $[272,357,537]$. Arachidonic acid ia a substrate for two enzymes: cyclooxygenase and lipoxygenase $[319,357,537]$. Cyclooxygenase converts arachidonate to prostaglandin endoperoxide $\mathrm{G}_{2}\left(\mathrm{PGG}_{2}\right)$, which is further reduced to $\mathrm{PGH}_{2}$. The endoperoxides $\mathrm{PGG}_{2}$ and $\mathrm{PGH}_{2}$ are unstable proaggregatory molecules. $\mathrm{PGH}_{2}$ is further metabolized by thromboxane synthase to $\operatorname{TxA}_{2}$, a powerful stimulatory platelet agonist. TXA2 is rapidly inactivated by hydrolysis to $\mathrm{TxB}_{2}$. Cyclooxygenase is irreversibly inhibited by aspirin. Lipoxygenase converts arachidonate to 12HPETE (12hydroperoxy-5,8,10,14-eicosatetraenoic acid), which is reduced to $12 \mathrm{HETE}$ (12-hydroxy$5,8,10,14$-eicosatetraenoic acid). Several reports suggest that lipoxygenase products may oppose the aggregating activity of endoperoxides and TXA2 [357].

Consistent with a central role of $\left[\mathrm{Ca}^{2+}\right]_{i}$ in platelet signal transduction, the regulation of $\mathrm{Ca}^{2+}$, in unstimulated platelets as well as in stimulated platelets, is intricately controlled by homeostatic mechanisms $[74,89,169,376]$. Cytosolic $\mathrm{Ca}^{2+}$ homeostasis is maintained by concerted operation of transport systems located in mitochondria, endoplasmic reticulum, and plasma membrane. Mitochondrial $\mathrm{Ca}^{2+}$ homeostasis is regulated by two transport systems: an energy-dependent uniport carrier and a less well-defined release mechanism involving a $\mathrm{H}^{+} / \mathrm{Ca}^{2+}$ exchange $[87,531]$. Mitochondrial $\mathrm{Ca}^{2+}$ pool is depleted upon oxidation of $\mathrm{NAD}(\mathrm{P}) \mathrm{H}$. Active transport of calcium ions into the DTS and to a lesser extent over the plasma membrane is achieved by two distinct $\mathrm{Ca}^{2+} / \mathrm{Mg}^{2}+-\mathrm{ATPases}[185,188,254]$. The $\mathrm{Ca}^{2+} / \mathrm{Mg}^{2}+-\mathrm{ATP}^{2}$ ases from the DTS is subject to bidirectional modulation by CAMP-dependent phosphorylation and $\mathrm{CaM} / \mathrm{Ca}^{2+}$-dependent phosphorylation $[5,376,719]$. In view of the inwolvement of free thiol groups in $\mathrm{Ca}^{2+}$ ATPase function [580], maintenance of the cellular redox status may be important for optimal $\mathrm{Ca}^{2+} / \mathrm{Mg}^{2+}-$ ATPase activity. Transport over the plasma membrane can also be achieved by a $\mathrm{Na}^{+} / \mathrm{Ca}^{2+}$ exchanger [528]. In activated platelets, additional $\mathrm{Ca}^{2+}$ transport systems are evoked: $\mathrm{Ca}^{2}$ + channels in the plasma membrane (ROC's) and the DTS. Influx of $\mathrm{Ca}^{2+}$ via ROC's is under negative feedback control of C-kinase $[169,395,533]$ and probably $\left[\mathrm{Ca}^{2+}{ }_{\mathrm{i}}\right.$. and may be modulated by $G$-proteins [81, 244]. Mobilization of $\mathrm{Ca}^{2+}$ from the DTS by $\mathrm{IP}_{3}$ is dampened by $\left[\mathrm{Ca}^{2+}\right]_{i}$ and suppressed by cAMP-dependent inhibition of phospholipase C $[263,395,581]$. 
In platelets, stimulatory pathways are antagonized by cAMP-dependent processes [581, 646]. CAMP is produced from ATP by adenylate cyclase, which is exclusively located at the cytosolic side of the platelet plasma membrane [418]. Adenylate cyclase activity is modulated by an inhibitory $\left(G_{i}\right)$ and stimulatory $\left(G_{\$}\right) G-$ protein $[235,236,381]$. Inhibitory platelet agonists (PGE1, PGI, and adenosine) stimulate adenylate cyclase: Excitatory agonists (thrombin, collagen, PAF) and proaggregatory agonists (ADP, epinephrine) inhibit adenylate cyclase [646]. The propagation of the inhibitory signal is ensured by cAMP-dependent kinase (A-kinase). Platelets contain two A-kinases: a membrane bound type $I_{\text {, }}$ and a soluble type II [231]. Important substrates of A-kinase are CAMP phosphodiesterase, filamin, and MLC kinase [231, 646]. cAMP phosphodiesterase actually is under bidirectional control: stimulation by A-kinase of provides an important negative feedback, which is abolished by increased $\left[\mathrm{Ca}^{2+}\right]$. Phosphorylation of MLC kinase by Akinase results in substantial inhibition of its ac- tivity towards MLC. Other substrates of A-kinase are two proteins with a molecular weight of $22 \mathrm{kDa}(\mathrm{P} 22)$ and $24 \mathrm{kDa}(\mathrm{P} 24)$. The function of these proteins and their cAMP-dependent phosphorylation is not clear at present. In addition to phosphorylation, stimulation of adenylate cyclase results also in dephosphorylation of MLC (P20) and P47, which is associated with disaggregation and cytoskeletal disassembly, and which may result from inhibition of phosphorylation by MLC kinase and C-kinase, or from stimulation of cAMP-dependent phosphatases. The most obvious effect of stimulation of adenylate cyclase is the inhibition of $\mathrm{Ca}^{2+}$ mobilization, both influx from extracellular $\mathrm{Ca}^{2+}$ and release from intracellular stores. The latter probably is a result of inhibition of phospholipase $C$ by increased cAMP levels [581]. An intriguing possibility arises regarding recent developments in G-protein research [81, $272,386,452]$. In view of newly discovered GTP-binding proteins in platelets $[55,193]$ in the range of $21-27 \mathrm{kDa}$, and in view of the regulation of $\mathrm{G}_{\alpha}$ subunits by phosphorylation, it may

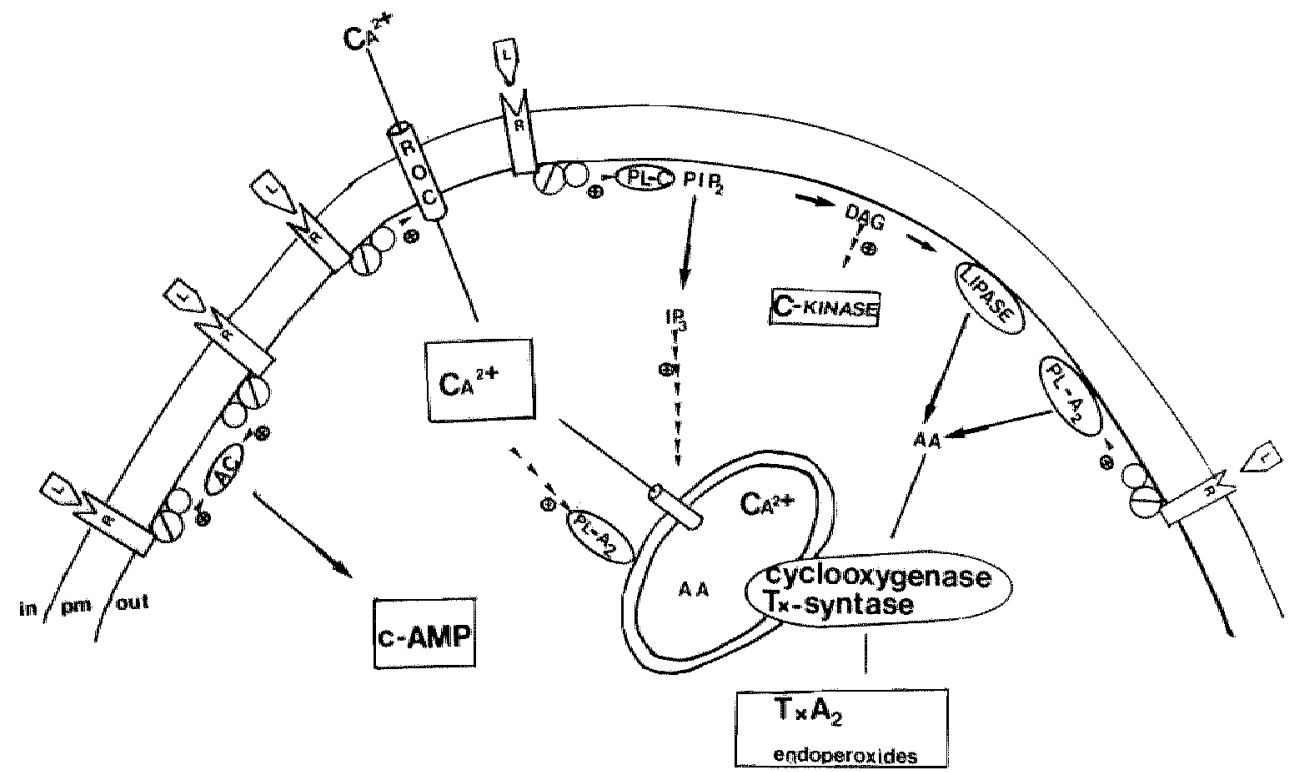

Fig. 5. Schematic representation of the platelet signal transduction system. For sake of clarity, only the production of second messengers is described. Inhibitory and stimulatory interactions between different systems and their target proteins are described throughout the text. As a general rule signal transduction starts with receptor (R) activation by agonistic ligands (L), and is mediated at the cytosolic side of the plasma membrane (PM) by G-proteins which stimulate key-enzymes to produce second messenger molecules. Stimulatory pathways inwolve phospholipase C (PL-C), phospholipase $\mathrm{A}_{2}$ (PL-A2) and receptor operated $\mathrm{Ca}^{2+}$ channels (ROC's). The inhibitory pathway involves adenylate cyclase (AC). Further abbreviations are defined in the text. Stimulation of ROC's and phospholipase $\mathrm{A}_{2}$ by G-proteins is still speculative, as well as the topography of the phospholipase A2 system. 
well be that CAMP-dependent phosphorylation of $\mathbf{P} 22$ and $\mathrm{P} 24$ is linked to inhibition of plasma membrane $\mathrm{Ca}^{2+}$ channels (ROC's) and phospholipase $\mathrm{C}$. Inhibition of $\mathrm{Ca}^{2+}$ release from the DTS may also result from CAMP dependent inhibition of $\mathrm{IP}_{3}$ induced $\mathrm{Ca}^{2+}$ release [187].

The function and significance of cGMP in platelets is unclear [646]. Platelets contain guanylate cyclase, a cGMP phosphodiesterase, but no cGMP-dependent kinase. Platelet guanylate cyclase is a soluble heme protein, which is de- pendent on the redox-status of the cell and which can be activated by stimulatory agonists via a $\mathrm{Ca}^{2+}$-dependent step, and by polyunsaturated fatty acids. The cGMP phosphodiesterase is stimulated by $\mathrm{PGE}_{1}$, suggesting control by inhibitory agonists. Interaction between the CAMP and CGMP system occurs mainly at the level of hydrolysis of each others cyclic nucleotides.

To reconcile platelet signal transduction, the main pathways are modeled in Fig. 5 .

\section{CALPAIN}

\section{Characteristics}

Calpain is an ubiquitous proteolytic enzyme (E. C. 3. 4. 22. 17), dependent on $\mathrm{Ca}^{2+}$ and with a thiol group in the active centre requiring neutral $\mathrm{pH}$ for its activity [433]. Based on its $\mathrm{Ca}^{2+}$-dependence, two subclasses of calpain are recognized; calpain-I and calpain-II, which show halfmaximal activity at $20-50 \mu \mathrm{M}$ and $0.2-0.5 \mathrm{mM} \mathrm{Ca}^{2+}$, respectively. Calpain is a heterodimer consisting of a small and large subunit $[624,652]$. The small subunit of $30 \mathrm{kDa}$ is similar for both calpain-I and calpain-II [179, $687]$, and functions as a regulatory subunit [179, 295, 312]. The large subunit (about $80 \mathrm{kDa}$ ), is different for calpain-I and calpain- $\mathbb{I}[180,687]$ and contains the active center [652]. Based on its amino-acid sequence, it is suggested that the large subunit arose from a gene fusion of calmodulin and a thiol protease [181]. Calpain is dependent on a reduced thiol group for full activity [625], and can be inhibited by $\mathrm{SH}-$ modifying agents (diamide, NEM, mersalylic acid) or by more specific oligopeptide-inhibitors like leupeptin and antipain. Calpain and its properties have been reviewed elsewhere [393, $394,431,432,433,511,514,573,622,624,627$, $682]$.

\section{Regulation}

It has long been thought that the relatively high $\mathrm{Ca}^{2+}$ requirement of calpain would ex- clude a role for calpain in intracellular mechanisms. Interest in calpain, however, has clearly revived considering the vast amount of literature concerned with various aspects of cellular calpain. This interest in calpain is probably related to the manifold manipulations of calpain activity occurring within cells, which suggest that calpain can be subject to fine-tuning of its activity, making a role in cellular function most likely.

Autoactivation has been suggested to lower the $\mathrm{Ca}^{2+}$-dependence of calpain-II, by removal of a small fragment from the heavy subunit $[130,276,626,628,629]$. As a result, an additional binding site with a much higher affinity for $\mathrm{Ca}^{2+}$ would be uncovered. The $\mathrm{Ca}^{2+}$ requirement for autoactivation of calpain-II can be reduced by binding to $\overline{\mathbf{P}}$ containing membranes [129, 295]. Autoactivation of calpain-I has also been reported [517], and is dependent on binding to the cytosolic side of the plasma membrane [346]. In this case an inactive pro-enzyme with a molecular weight of several hundred $\mathrm{kDa}$ is transformed into calpain-I, without any change in affinity for $\mathrm{Ca}^{2+}$.

Just as the positive feedback via autoactivation, calpain is also subject to negative feedback via autolysis, rendering calpain inactive [296]. This property is shared by both calpain-1 and - II, and may be an important way to terminate the action of calpain thereby allowing spatially and temporally localized protein degradation by calpain.

Calpastatin is an endogenous inhibitor of calpain found in all cells where calpain is pres- 
ent [431, 432, 433]. Calpastatin can bind to membranes [416]. The molecular weight differs greatly among vartes sources, which may be caused by different degrees of polymerization. Regulation of calpain by calpastatin may turn out to be very complex. Calpastatin has been found to keep inactivate calpain independent of $\mathrm{Ca}^{2+}[133]$, or to inhibit calpain after binding of $\mathrm{Ca}^{2+}$ [134]. Calpastatin can inhibit binding of calpain to membranes [517]. In addition, calpastatin itself is a substrate for calpain [417]. Calpastatin does not discriminate between calpain-I and -II [431].

The major determinant of the relevance of calpastatin in a particular cell may be the quamitative balance between calpain and calpastatin [431, 432, 433]. In erythrocytes, which contain calpastatin in excess of calpain-1 [241], treatment with a $\mathrm{Ca}^{2+}$ ionophore in the presence of millimolar $\mathrm{Ca}^{2+}$ does only lead to cytoskeletal degradation after prolonged incubations $[95,248,389]$, or after addition of purified calpain [140]. In contrast, calpain can easily be activated by ionophore treatment in platelets $[124,664,690]$, which contain calpain-I in excess of calpastatin [431, 432, 433].

Binding of calpain to membranes is facilitated by the small subunit [295]. This subunit contains a high amount of hydrophobic amino acids at the $\mathrm{N}$-terminus, while the $\mathrm{C}$-terminus represents a $\mathrm{Ca}^{2+}$ binding region [179], suggesting that membrane binding can be regulated by $\mathrm{Ca}^{2+}$. Binding of calpain to membranes has been reported to be dependent on anionic phospholipids like PS and PI [129]. In resting cells, calpain is present in a soluble form in the cytosol. This would meran that during cell activation translocation of calpain to the plasma membrane may occur. Membrane binding of calpain may be an important way to limit the action of calpain to membrane surfaces [415], or to localize calpain near its substrates.

Calpain can be phosphorylated in vitro by protein kinase C [283]. Phosphorylation only involves inactive calpain, suggesting a regulatory role for phosphorylation. In vivo, phosphorylation of calpain could not be demonstrated [1]. Thus, the importance of phosphorylation in regulation of calpain acitivity is at present unclear.

In Triton-insoluble residues from bovine brain [156,633], platelets [633], and neutrophils [515], a $20-40 \mathrm{kDa}$ activator of calpain has been found. This activator increased calpain activity without changing its sensitivity for $\mathrm{Ca}^{2+}$. The relevance of this small protein for regulation of calpain acitivity is still unclear. The effect of the activator on calpain can be antagonized by call pastatin [515], which suggests that calpain may be modulated in vivo by dynamic interaction between activator and inhibitor.

In principle, enzyme activity can be dependent on the structure of its substrate. Evidence is accumulating that phosphorylation of substrate proteins modulates their susceptibility towards calpain. Phosphorylation of filamin prevents degradation by calpain [723]. Phosphorylation of cytoskeletal proteins by C-kinase in neutrophils [512] and cAMP-dependent phosphorylation of troponin [645] triggers their degradation by calpain. Substrate modulation may be a common way of restricting calpain activity to particular pools of substrates. The main substrates of calpain (vide infra) are subject to structural changes upon cell activation.

\section{Substrates}

Substirates of calpain fall in two main categories: cytoskeletal proteins and membrane proteins. In fact, a common denominator of both categories may be the binding of calpain to membrames. In general, proteolysis by calpain is characterized by its limited action, normally producing only few fragments. The substrate specificities of calpain-I and calpain-II are quite alike but not identical. Both calpains prefer an Arg or Lys preceded by a Leu or Val [431].

Cytoskeletal substrates of calpain cover the whole range of cytoskeletal structures: microtubules, intermediate filaments, and actin filaments. Both tubulin [58] and microtubule-associated proteins $[58,563]$ can be degraded by calpain. Intermediate filaments (vimentin, desmin) $[453,493,570]$ and neurofilament proteins $[464,485,672]$ are documented substrates for calpain. Many actin-binding proteins can be hydrolysed by calpain: spectrin $[215,296]$, ankyrin [262], band-4. 1 [140], fodrin [393, 463, $672]$, filamin [212], talin $[30,31,122,212,470$, 690], myosin [296], $\alpha$-actinin [717], caldesmon [675], tropomyosin [682], and vinculin [280]. Most peculiar, actin is not degraded by calpain 
[679], suggesting that the action of calpain is not ment to destroy the cytoskeleton but restricted to change its organization. Limited hydrolysis of the high-molecular weight actinbinding proteins results mostly in cleavage of a small fragment. In case of filamin, the large fragment is still able to bind actin [93], but has lost its capacity to crosslink actin filaments $\llbracket 150$, $151,648]$, again suggesting that limited proteolysis of cytoskeletal proteins must lead to reorganization but not destruction of the cytoskeleton. This is further documented by the observation that the interaction of filamin with the actomyosin ATPase $[143,475,610]$ is abolished after limited proteolysis by calpain [474]. In some cells $[464,664,690]$ millimolar $\mathrm{Ca}^{2+}$ calpain is able to degrade cytoskeletal proteins to many small fragments in the presence of milllimolar $\mathrm{Ca}^{2+}$.

Membrane-bound substrates of calpain can be further divided in two classes: transmembrane receptors and cytosolic proteins. Limited proteolysis by calpain can modulate several receptors: epidermal growth factor receptor $[227,251]$, platelet derived growth factor receptor [177], glutamate receptor [599], and insulin receptor [375]. As a result, receptor properties will be changed, like autophosphorylation, coupling to cytoskeletal proteins or ligand-binding characteristics. Calpain can also affect several key proteins in signal transduction and cellular $\mathrm{Ca}^{2+}$ homeostasis. Adenylate cyclase $[4,647], C$-kinase $[252,283,323,414$, $512,513,515,516,565,637]$, A-kinase [553], phospholipase C [390], CaM-dependent phosphatase [675], MLC kinase [306, 307, 675], and $\mathrm{Ca}^{2+} / \mathrm{Mg}^{2+}$-ATPase $[20,678]$ become irreversibly activated upon limited protelysis by calpain.

A number of extracellular substrates have been described. Among these are the platelet glycoproteins $I_{b}[411,713]$ and $V[56,411]$, fibrinogen [351], won Willebrand factor [352], and high molecular weight kininogen [571]. In addition, several proteins involved in coagulation can be hydrolysed by calpain: factor V [69], factor XIII [11], and protein S [421]. However, in intact cells calpain is confined to the cytosol $[431,555]$, suggesting that extracellular substrates are onily relevant in case of cell damage.

\section{Function}

Stimulation of calpain leads to irreversible changes in protein structure and function. Thus, any function of calpain in cellular processes is likely to be dependent on this irreversibility, and would involve long-term changes. Although the function of calpain in cellular processes is only beginning to be understand, some examples have been investigated in more detail. In brain, calpain-I mediated degradation of fodrin (brain spectrin) increases irreversibly the number of functional glutamate receptors, thereby providing a plausible mechanism for long-term memory $[393,394]$. In chicken erythropolesis, calpain is involved in the construction of the membrane skeleton, by eliminating unused cytoskeletal proteins tagged by posttranslationall modification [372, 708]. In erythrocytes $[7,241,242]$ and myoblasts $[574,575]$, calpain has been suggested to play a role in membrane fusion by clearing part of the membrane from the underlying network of cytoskeletal proteins. Calpain may be involved in cellular destruction, considering the massive proteolysis during myocardial ischemia [24, $255,277,374,644,651]$, and myelopathy [23]. Finally, calpain may be involved in the regulation of the microtubular reorganization during mitosis $[252,573]$. Obviously, the functions described are in accordance with the substrate specificity of calpain: cytoskeletal proteins apparently are the primary targets. The listed functions, however, lack examples of involvement of calpain in signal transduction, despite ample indications that such an involvenent would be feasible (vide supra).

\section{Calpain in platelets}

In platelets, calpain has been subject of extensive research since its discovery in 1977 [499]. Platelet calpain mainily consists of calpain-I $[212,333,552,655,679]$, and is a cytosolic protein [555] responsible for almost all $\mathrm{Ca}^{2+}$-dependent proteolytic activity in platelets $[411,499,648]$. Calpastatin is present in p].atelets only in minor amounts $[431,433,630]$. A low molecular weight activator of calpain has been found in plateletts [633]. 
Platelet calpain activity can easily be monitored by the degradation of its major substrates and the formation of their fragments as judged from protein patterns after gel electrophoresis of reduced protein samples (Fig. 6). The major substrates of calpain are the cytoskeletal proteins filamin, talin, and myosin[124, 216, 499 , $608,656,690$ ]. Their major fragments are found at $190 \mathrm{kDa}$ (fragment 1), $135 \mathrm{kDa}$ (fragment 2), $93 \mathrm{kDa}$ (fragment 3 ); and at $48 \mathrm{kDa}$ (fragment 4) $[119,124,21.6,442,499,608,690]$. Filamin is degraded by calpain into fragment 1 and fragment 3 [212, 648]. Fragment 2 most likely represents the myosin rod cleaved from heavy chain myosin [296, 492]. Talin is degraded by calpain to a protein band corresponding with fragment 1 , indistinguishable from that of filamin, and to fragment $4[31,122,212,292,470]$. Platelet $\alpha$-actinin can also be hydrolysed by calpain $[522,717]$.

Degradation of filamin results in a decoupling of glycoprotein $I_{b}$ from the membrane skeleton $[206,207,472,608]$, and a loss of the actin-gelating capacity of filamin $[150,151$,
648]. Decoupling of glycoprotein Ib from the membrane skeleton results in a reorganization of this glycoprotein in the plasma membrane, and changes in its receptor properties. Degradation of talin may be related to a reduction in high affinity binding sites for fibrinogen on the glycoprotein $\mathrm{I}_{\mathrm{b}} / \mathrm{III}_{\text {a }}$ complex [490]. Several proteins involved in signal transduction can become irreversibly activated by calpain: adenylate cyclase $[4,647], C$-kinase $[323,636]$, phospholipase C [390], and MLC kinase [306, $307,675]$.

Limited calpain activity has been observed in thrombin-activated platelets, only under stirring conditions and several minutes after addition of thrombin $[208,212]$. It has been suggested that this limited degradation of cytoskeletal proteins is a prerequisite for the formation of a contractile skeleton. Stimulation of platelets by the $\mathrm{Ca}^{2+}$ ionophore does result in massive degradation of cytoskeletal proteins $[124,664,690]$, indicative of the potency of platelet calpain.

\section{REGULATION OF TRANSBILAYER LIPID ASYMMETRY}

\section{Transbilayer movement in lipid model systems}

A lipid bilayer is generally a stable structure held together due to thermodynamically and hydrodynamically fawoured interaction between amphipathic lipids $[164,298,549]$. Transbilayer movement of phospholipids (flipflop) would require the charged and hydrated headgroup to pass the hydrophobic bilayer interior. This process is characterized by a high activation energy. Thus, flipflop in stable unilamellar vesicles is a relatively slow process, occurring at time scales of days to weeks. Flipflop can be considerably enhanced by introduction of local defects in lipid bilayer packing [154].

Introduction of different physical properties between the two monolayers of a lipid membrane has been shown to induce enhanced rates of flipflop (fliprates). Differences in physical properties between monolayers include differences in headgroup composition [339], differences in phase behavior [344], and differences in fluidity [343]. Difference in headgroup composition refers to an asymmetric distribution of phospholipids, i.e. a concentration gradient over the membrane. Such a concentration gradient increases the free energy of lipids, and will allow more lipids to pass the threshold of activation energy for flipflop, resulting in higher fliprates. Differences in headgroup composition between the two monolayers of a membrane may also lead to formation of lipid bilayer instabilities (vide infra). Induction of a phase-separation in unilamellar vesicles of PC results in enhanced fliprates, as a result of packing defects at the border of gel-liquid crystalline phase transition [344]. Differences in physical properties between both monolayers 
of a membrane may find a biological correlate in the transbilayer fluidity gradient, which has been observed in most cells in which it was investigated $[318,429,587]$.

Insertion of transmembrane proteins may result in increased fliprates $[233,612,726]$. Incorporation in artificial vesicles of glycophorin and band -3 , membrane proteins isolated from the erythrocyte, substantially increases the fliprate of lyso-PC [726]. This is most likely due to the inability of a single phospholipid species to accommodate the irregularities at the interface of the membrane spanning proteins and the lipid bilayer, since more complex lipid mixtures, like total erythrocyte lipids, do not exhibit enhanced fliprates upon incorporation of glycophorin [233]. Most interesting is the observation that clustering of glycophorin in PS/PE vesicles by wheat germ agglutinin results in destabilization of the lipid bilayer and hence increased fliprates of phospholipids [612]. Ligand-dependent clustering of membrane glycoproteins is a common phenomenon in cells, and may thus constitute a biological mechanism to increase the fliprate in plasma membranes.

A subject of extensive research is the formation of non-billayer structures in model as well as in biological membranes $[141,341]$. Formation of lipidic structures is related to

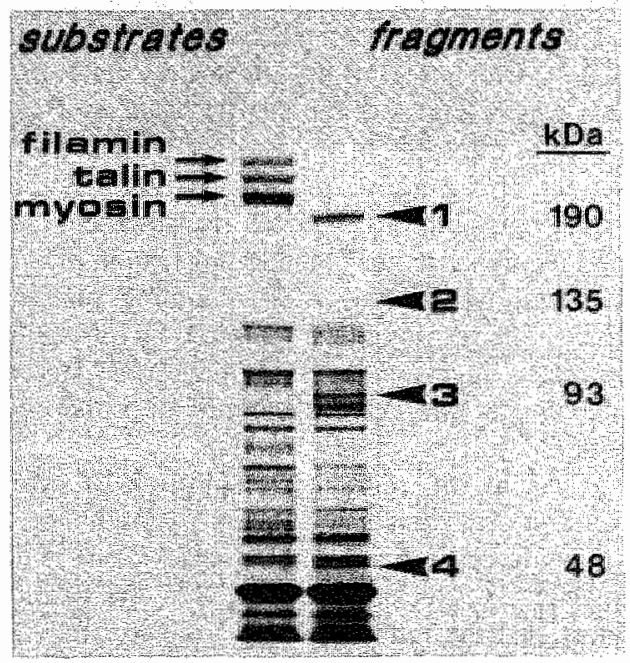

Fig. 6. Degradation of platelet cytoskeletal proteins by calpain. Numbered arrows indicate charactistic fragments, which are described in the text. Protein patterns were obtained before and after stimulation of human platelets by the $\mathrm{Ca}^{2+}$ ionophore $\mathrm{A} 23187$ in the presence of millimolar extracellulair $\mathrm{Ca}^{2+}$. For technical details is referred to chapter 2 . their molecular shape. Cylindrical phospholipids like PC and SphM will form lipid bilayers upon hydration. Lipids like PA, PE with unsaturated acyl chains, and diacylglycerides have a cone-like shape, and will adopt hexagonal structures upon hydration, such as tubes and inverted micelles with the acyl chains pointing outward. These lipids are abundant components of biological membranes, and have been implicated in membrane fusion and flipflop. Generally, the presence of non-bilayer lipids in membranes does not necessarilly result in disturbances of the bilayer structure $[234,642]$. This is due to the presence of other lipid species counteracting the formation of non-bilayer structures. However, if the lipids become heterogenously distributed in the membrane, domains of non-bilayer lipids may form and non-bilayer structures will occur [234, 642]. In this way, formation of non-bilayer structures can be under control of potentially physiological mechanisms, like clustering of PS by increased $\mathrm{Ca}^{2+}$ levels $[234,642]$, or clustering of transmembrane proteins [612].

The relevance of non-bilayer lipidic structures, such as inverted micelles, for enhanced fliprates can be questioned, because these structures apparently represent a thermodynamically favoured situation. However, since the fraction of phospholipids participating in fast isotropic motion, as observed by ${ }^{31}$ P NMR, is much larger than the fraction participating in non-bilayer structures, as observed by freeze fracture electron microscopy, it has been argued that rapid exchange between billayer and non-bilayer lipid occurs [342], thereby providing means for rapid transbilayer movement of phospholipids. Involvement of non-bilayer structures in rapid flipflop is further suggested by their relation in modell systems [172], as well as biological membranes [171], though it may well be that non-bilayer structures are not necessarily involved in rapid transbillayer movement of phospholipids in biological membranes [107]. Formation of non-bilayer structures in biological membranes may be facilitated by the composition and distribution of the phospholipids. Concentration of $\mathrm{PE}$ with a large amount of polyunsaturated acyl chains at the cytosolic side of the plasma membrane will favour formation of non-bilayer structures under certain conditions, like increased $\mathrm{Ca}^{2+}$ levels [291]. 


\section{Transbilayer movement in biological} membranes

Regullation of transbillayer lipid asymmetry in biological membranes has been subject of several reviews $[476,477,479,545,577]$, and is best studied in the erythrocyte. Erythrocytes have only one membrane, the plasma membrane. The phospholipids are asymmetrically distributed over the plasma membrane, quite similar as in platelets: choline-phospholipids and amino-phospholipids are enriched in the outer and inner leaflet, respectively [404, 666, 736]. The lifespan of erythrocytes, about 120 days, is much longer than that for platelets. This long lifespan has lead to numerous investigations of the mechanisms by which transbilayer lipid asymmetry is maintained. Originally these investigations focussed on the role of the membrane skeleton $[16,39,104,168,217,256,349$, $423,424,572,703,704]$. More recently, however, interest has changed focus on the role of a phospholipid transport protein [127, 144, 281, $406,585,643,702,721]$.

The cytoskeleton of erythrocytes covers the cytosolic face of the plasma membrane, and was the first example of a membrane skeleton. The membrane skeleton is a network of spectrin and actin, which is coupled to the plasma membrane by binding to integral membrane proteins [reviewed in: $36,37,71,114,245,256,392$, $401,422,483,484,595,669,704,716]$. The suggestion that the membrane skeleton could be involved in the maintenance of transbilayer lipid asymmetry, came from experiments which demonstrated an interaction between spectrin and lipids of the cytosolic leaflet of the plasma membrane $[425,426]$, and experiments in which crosslinking of spectrin resulted in loss of transbillayer lipid asymmetry $[257,259]$. Structural modifications of the membrane skeleton, either in vitro or in pathological erythrocytes, further confirmed the notion that anionic phospholipids were hold at the cytosolic leaflet of the pllasma membrane by direct interaction with spectrin $[16,39,104,168,217,256,349,423$, $424,572,703,704]$. Direct interaction between erythrocyte cytoskeletal proteins and membrane lipids has been frequently observed by now, not only for spectrin $[64,112,398,425$, $426,674]$ but also for band 4. $1[113,564,596$, 674 , a protein that links the membrane skeleton to the erythrocyte plasma membrane. In general, interaction between cytoskeletal proteins and the lipid bilayer is dependent on electrostatic attraction, followed by consolidation of binding by additional hydrophobic interactions.

Structural disturbance of the erythrocyte membrane skeleton may not necessarily result in net transbilayer movement of PS, but it generally leads to enhanced rates of transbilayer movement. Thus it is observed that exogenously added lyso-PC or endogenous PC become subject to increased fliprates whenever the structure of the membrane skeleton is damaged $[218,219,220,258,354,477]$. Apparently, the structural integrity of the lipid bilayer is dependent on an intact membrane skelleton. This suggestion is supported by results from direct measurements of membrane structure by lipidic probes $[15,95,103,104$, $106,194,408,526,703]$.

Application of various phospholipid probes to erythrocytes clearly demonstrated the occurrence of rapid transbilayer movement from the outer to the inner leaflet of the plasma membrane, specific for $P S$, and to a lesser extent for PE $[144,406,585,643,721]$. This transbilayer movement is dependent on intracellular ATP levels, and is characterized by a halftime of approximately 7 minutes for PS, which is much higher than passive rates of transbilayer movement through lipid bilayers. The phospholipid transport activity is inhibited by thiol oxidizing agents, which lead to the suggestion that a protein was responsible for the observed ATP-dependent transport of PS over the plasma membrane. This putative protein has been named amünophospholipid specific translocase [721], and its presence has been demonstrated in various cell types $[406,722]$, including platelets [623]. The translocase is inhibited by intracellular $\mathrm{Ca}^{2+}$ levels above $1 \mu \mathrm{M}[721$ ], and stereospecifically interacts with amino-phospholipids [406].

As to the relative contribution of cytoskeleton and translocase in the maintenance of transbilayer lipid asymmetry, conscensus is not reached. It has been argued that the transbilayer distribution of PS and PE can solely be explained by the ratio between inward and outward rate-constants of the translocase [281], whille others argue that a translocase with identical outward and inward rate-constants in cooperation with selective binding of aminophospholipids to the membrane skeleton are responsible for the observed phospholipids 
transbilayer asymmetry [702]. It seems most likely, considering the ample evidence for $d:-$ rect interactions between membrane lipids and cytoskeletal proteins, that the membrane skeleton is involved in the maintenance of phospholipid transbilayer asymmetry, e. g. by maintaining the structural properties of the lipid bilayer.

With respect to the loss of transbilayer lipid asymmetry, the erythrocyte does not give definite clues. Original findings that increased fliprates after manipulation of cytoskeletal organization did not result in exposure of PS, were taken as evidence for selective interaction of PS with cytoskeletal proteins [vide supra]. This conclusion may have been premature, since an alternative explanation would be the selective action of a translocase. Recent experi- ments in which both cytoskeletal structure and translocase function were individually manipulated [420], however, seem to support the original assumption that cytoskeletal proteins contribute to the maintenance of phospholipid transbilayer asymmetry by binding anionic phospholipids at the cytosolic leaflet of the plasma membrane.

With respect to the loss of transbilayer lipid asymmetry during platelet activation, the most relevant observation probably is that a decreased interaction between cytoskeletal proteins and the plasma membrane increases the rate of transbilayer movement over the plasma membrane [vide supra], resulting in a loss of transbilayer lipid asymmetry in the absence of any other regulatory factor.

\section{PLATELET PROCOAGULANT ACTIVITY}

\section{Introduction}

The interaction of various coagulation factors with activated platelets plays an essential role in hemostatic plug formation. In particular, the platelet surface membrane interacts with factors $X_{a}$ and $V_{a}$ to ensure formation of the prothrombinase complex, as well as promoting the assembly of the factor IX $\mathrm{X}_{\mathrm{a}}-\mathrm{VIII}_{\mathrm{a}}$ complex required for activation of factor $X$. Binding to activated platelets dramatically enhances the enzymatic activity of these complexes of coagulation factors, in a manner resembling the catalytic activity of negatively charged phospholipid surfaces [543a]. At the lipid bilayer surface, binding of factors $I X, X$, and prothrombin is dependent on $\mathrm{Ca}^{2+}$, which forms a complex between negative charges on the phospholipid molecules and $\gamma$-carboxyglutamic acid residues present in these coagulation factors. The nonenzymatic protein cofactors $\mathrm{V}_{\mathrm{a}}$ and $\mathrm{VIII}_{\mathrm{a}}$ are thought to interact with lipids by penetration into the hydrophobic membrane interior. This interaction does not depend on $\mathrm{Ca}^{2+}$ but requires negatively charged phospholipids. Various aspects of platelet procoagulant activity have been reviewed elsewhere $[48,53,54,543 \mathrm{a}$, $728,729,731,732,733,735,737]$

\section{Prothrombin and factor $\mathrm{X}$ activation by platelets}

The stimulatory effect of various platelet preparations in prothrombin and factor $\mathrm{X}$ activation (procoagulant activity) has been compared, using assay systems with highly purified coagulation factors and specific chromogenic substrates to measure rates of thrombin and factor $X$ formation (Table I) $[49,50,530,543 a]$. ADP, PAF, serotonin, epinephrine and arachidonic acid have virtually no effect on procoagulant activity, and thrombin shows not more than a twofold increase in procoagulant activity relative to control platelets. Collagen is the only single physiological agonist capable of inducing a substantial increase in the capacity of platelets to stimulate prothrombin and factor $\mathrm{X}$ activation. Highest physiological stimulation, however, is achieved by the combined action of collagen and thrombin. The most potent stimulator of platelet procoagulant activity is the $\mathrm{Ca}^{2+}$ ionophore A23187. Treatment with diamide, an SH oxidizing compound, also renders platelets procoagulant. In contrast to other activators , this occurs at diamide concentrations where release and aggregation are no longer observed. The highest increase in pro- 
coagulant activity is observed when platellets are completely lysed. The prothrombinase activity of lysed platelets equals that of their lipid extracts suggesting that no components other than phospholipids are responsible for this effect. The notion that phospholipids at the outer surface of activated platelets are responsible for the observed procoagulant activities is further supported by the observation that treatment of platelets with phospholipase $\mathrm{A}_{2}$ from $N$. naja abolishes their procoagulant activity [51].

Expression of functional binding sites for coagulation factors on the platelet surface requires extracellular $\mathrm{Ca}^{2+}$ during platelet activation since no procoagulant activity is produced in the absence of $\mathrm{Ca}^{2+}$ or in the presence of $\mathrm{Ca}^{2+}$-channel blockers dilthiazem and verapamil [52]. Treatment of platelets with prostacyclin or dibutyryl-cAMP, which stimulate CAMP-dependent processes, inhibits stimulation of procoagulant activity by collagen plus thrombin [734]. Aspirin, however, does not inhibit expression of procoagulant activity, indicating that thromboxane $A_{2}$ formation is not essential [734].

Stimulation of platelet procoagulant activity by the combined action of collagen and thrombin is dependent on certain properties of both agonists. Both collagen type I and type III are effective, and their activity can be abolished by a collagen-derived octapeptide, suggesting the requirement of specific ligand-receptor interactions [52]. The contribution of thrombin to the stimulation of procoagulant activity by collagen plus thrombin is dependent on its hydrolytic capacity. DFP-inhibited thrombin displays normal binding to platelets but lost its proteolytic activity, and is not effective in combination with collagen [730]. In the combination collagen plus thrombin, thrombin can be replaced by trypsin or a-clostripain without significant loss of procoagulant activity [730], supporting the notion that proteolysis of specific membrane proteins determines the synergistic activity of thrombin.

\section{Changes in phospholipid exposure upon platelet activation}

Determination of the orientation of phospholipids in plasma membranes is based on the application of non-permeable lipid-reactive probes, which allow identification of the amount and possibly the lippid composition in the outer monolayer of the plasma membrane $[192,338,476]$. Exogenous phospholipases are such probes, which have the advantage over chemical reagents that a proper combination of them can react with all phospholipid classes and that reaction goes to completion $[539,540]$.

Determination of the maximal amount as well as the composition of phospholipids that can be degraded in the outer monolayer of the platelet plasma membrane has been achieved by a mixture of Sphingomyelinase and phospholipase $A_{2}[49,50,51]$. With control platelets some $21 \%$ of the total phospholipids can be degraded in the absence of cell lysis, representing the phospholipid fraction in the external leaflet of the plasma membrane. This fraction is mainly composed of choline-phospholipids (SphM and PC), whereas degradation of amino-phospholipids (particulary PS) is lirnited (Table II). Activation of platelets by collagen or thrombin followed by addition of phospho-

Table I. Efrect of different platelet treatments on their procoagulant activity, as expressed by activation of prothrombin and factor $X$.

\begin{tabular}{lcc}
\hline $\begin{array}{l}\text { platelet } \\
\text { treatment }\end{array}$ & $\begin{array}{c}\text { prothrombin } \\
\text { activation }\end{array}$ & $\begin{array}{c}\text { factor X } \\
\text { activation }\end{array}$ \\
\hline none & 34 & 2 \\
ADP & 36 & 2 \\
thrombin & 40 & 3 \\
collagen & 98 & 18 \\
collagen + thrombin & 351 & 47 \\
diamide & 356 & 45 \\
A23187 & 843 & 94 \\
sonication & 1170 & 149 \\
& & \\
collagen + thrombin & 17 & 1 \\
followed by & & \\
phospholipase A2 & &
\end{tabular}

Prothrombin activation and factor $\mathrm{X}$ activation are eXpressed as $\mathrm{nM}$ thrombin per min and $\mathrm{nM} X_{\mathrm{a}}$ per min, respectively. Data were obtained using parified coagulation factors under conditions were the procoagulant activity of platelets is rate limiting. Treatment specifications: 10 $\mu \mathrm{g} / \mathrm{ml}$ collaggen, $2 \mathrm{nM}$ thrombin, $5 \mathrm{mM}$ diamide, $1 \mu \mathrm{M}$ A23187, 3 min direct-probe sonication. Data were taken from $[50,543 a]$. 
served when platelets are treated with A23187 before application of phospholipases. Remarkably, some $65 \%$ of all platelet phospholipids can be degraded in ionophore-treated platelets before the onset of cell lysis.

Changes in phospholipid composition of the outer membrane leaflet of platelets activated by thrombin are mainly caused by the release reaction, as a result of fusion of granular membranes with the plasma membrane. On the other hand, increased exposure of PS accompanied by decreased exposure of SphM in platelets activated by collagen plus thrombin, diamide, or $\mathrm{A23187}$, presumably results from an enhanced transbilayer movement of phospholipids during platelet activation.

Since PS is the major anionic phospholipid in platelet membranes, its increased localization in the outer leaflet of the plasma membrane could be the cause of the increased procoagulant activity. When the percentage of total PS exposed at the outer surface of activated platelets is compared to the prothrombinase activity of the same activated platelets, the relation between exposure of PS and platelet procoagulant activity becomes evident (Fig. 7).

\section{Prothrombinase activity of pathological platelets.}

The ability of platelets to alter their membrane phospholipid orientation upon platelet activation, can be expected to require the participation of other platelet components or processes. Identification of these additional requirements may be derived from studies with different pathological platelets [48], since most of these are based on well-defined molecular or structural defects. Platelets which lack either a-granules (Gray platelet syndrome) or dense granules (Hermansky Pudlak syndrome) cannot be distinguished from normal platelets with respect to their activation-dependent procoagilant activity. This indicates that the release reaction may not be a prerequisite for the expression of platelet procongulant activity. In view of the inability of thrombin to induce exposure of PS whille complete release is achieved, it is suggested that the release reaction is not responsible for enhanced transbilayer movement of phospholipids in activated platelets.

Besides release, also platelet aggregation does not seem to be required for inducing a procoagulant surface. Platelets obtained from patients with Glanzmann's thrombasthenia fail

Table II. Stimulation-dependent changes in phospholipid composition of the outer monolayer of the platelet plasma membrane as determined by phospholipase treatment.

\begin{tabular}{lccccccc}
\hline $\begin{array}{l}\text { phospholipid } \\
\text { class }\end{array}$ & unstimulated thrombin & collager & $\begin{array}{c}\text { collagen } \\
+ \text { thrombin }\end{array}$ & diamide & A23187 \\
PS & $2(4)$ & $5(15)$ & $6(19)$ & $11(46)$ & $13(49)$ & $13(75)$ \\
PE & $10(7)$ & $16(19)$ & $17(22)$ & $36(60)$ & $26(38)$ & $36(87)$ \\
PC & $31(17)$ & $39(34)$ & $41(37)$ & $34(39)$ & $39(41)$ & $30(50)$ \\
SphM & $57(63)$ & $40(69)$ & $36(65)$ & $19(43)$ & $22(45)$ & $22(75)$ \\
hydrolyzed & 21 & 33 & 35 & 45 & 40 & 65 \\
fraction & & & & & & &
\end{tabular}

Values are expressed as percentage of the fraction of total phospholipids which is hydrolyzed by the combined action of exogenous phospholipase $\mathrm{A}_{2}$ and sphingonyelinase. This fraction represents the outer monolayer of the plasma membrane under non-lytic conditions. Relative standard devations range from $20 \%$ for unistimulated platelets and platelets stimulated by thrombin ( $2 \mathrm{nM}$ ) or collagen $(10 \mu \mathrm{g} / \mathrm{ml})$, to $10 \%$ for platelets treated with thrombin and colagen, diamide $(5 \mathrm{mM})$, and $\mathrm{A} 23187(1 \mu \mathrm{M})$. Valoes in parenthesis refer to the percent degradation of the whole phospholipid class. The contribution of PI ( $3 \%$ of total phospholipids) in addition to PS (12\% of total phospholipids) is neglected. Abbreviations ane explained in the text. Data are taken from [50]. 
be distinguished from normal platelets with respect to their activation-dependent procoagulant activity. This indicates that the release reaction may not be a prerequisite for the expression of platelet procoagulant activity. In view of the inability of thrombin to induce exposure of PS while complete release is achieved, it is suggested that the release reaction is not responsible for enhanced transbilayer movement of phospholipids in activated platelets.

Besides release, also platelet aggregation does not seem to be required for inducing a procoagullant surface. Platelets obtained from patients with Glanzmann's thrombasthenia fail to aggregate upon addition of collagen plus thrombin, but exhibit a similar increase in prothrombinase activity as observed with normal platelets. Since these platellets show partial or complete deficiencies in membrane glycoproteins $\mathrm{I}_{\mathrm{b}}$ and $\mathrm{II}_{\mathrm{a}}$, the involvement of these proteins in mediating transbilayer movement of phospholipids over the platelet plasma membrane can be excluded.

Platelets from patients with Bernard-Soulier syndrome exhibit a particular interesting behavior regarding their procoagulant activity. In the unstimulated form, they are significantly more active than normal platelets, which cannot be explained by their increased size only. Bernard-Soulier platelets display abnormal phospholipid transbilayer asymmetry: the relative content of PS in the outer leaflet of the plasma membrane is higher than in normal platelets. This would explain the abnormally high basal level of procoagulant activity. Upon stimulation by collagen plus thrombin, procoagulant activity of Bernard-Soulier platelets increases towards similar levels as in normal platelets. Bernard-Soulier platelets are deficient in glycoprotein Ib and V. In addition the abnormally large size of these platelets may point to defects in cytoskeletal organization. Thus, cytoskeleton and glycoprotein Ib may be involved in maintaining low levels of procoagulant activity in unstimulated platelets.

Platelets lacking the glycoprotein $\mathrm{I}_{\mathrm{a}}$ fail to respond to collagen with aggregation and release [455]. In addition, stimulation of these platelets by the combined action of collagen and thrombin did not increase procoagulant activity beyond basal levels, while the $\mathrm{Ca}^{2+}$ ionophore produced high levels of procoagulant activity as normal (unpublished results). It is con- cluded that in these glycoprotein $\mathbb{I}_{a}$ deficient platelets the potency to develop a procoagulant surface is not diminshed, but that stimulation of platelet procoagulant activity by collagen is inhibited. Thus, it is suggested that platelet glycoprotein $\mathbb{I}_{a}$ is important for collagen to induce procoagulant activity.

Most interestingly are results obtained from a patient with a moderately severe bleeding disorder, described as an isolated deficiency of platelet procoagulant activity [685]. Stimulation of these platelets resulted in a much lower number of binding sites for the prothrombinase complex as well as for the factor $\mathrm{X}$ activating complex than with normal platelets [543]. Incubations with phospholipases suggest a reduced exposure of PS upon platelet activation. This would point to a defect in the mechanism by which anionic phospholipids become exposed at the outer surface during platelet activation.

\section{Possible mechanism of PS exposure.}

Three possibilities can be distinguished which could explain the increased exposure of PS at the outer surface of activated platelets. First, PS may already be present at the outer

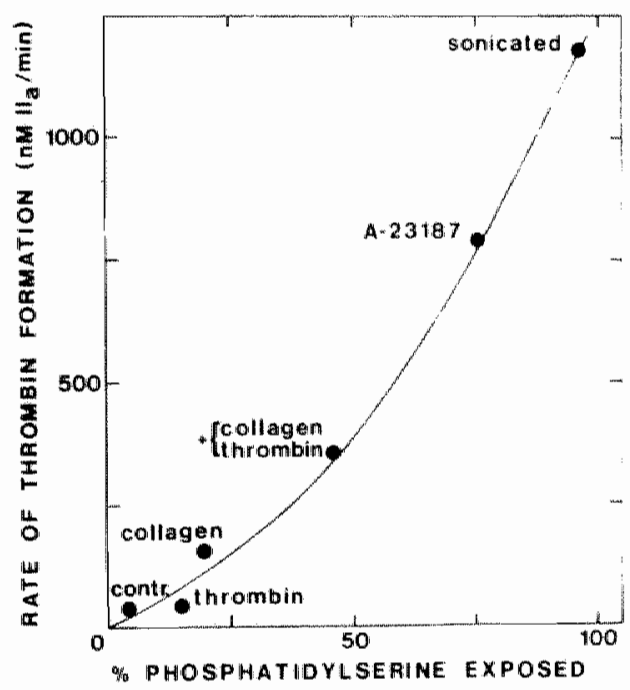

Fig. 7. Relation between prothrombinase activity and exposure of phosphatidylserine in activated human platelets. Each point represents the results from a different stimulation procedure. Unstimulated platelets are indicated by contr. 
surface, but not available to chemical probes and proteins like coagulation factors and phospholipases, e.g. by interaction with membrane proteins. However, proteolytic treatment of platelets does not increase the availability of PS for chemical probes. Moreover, close to half of the plasma membrane phospholipids can be hydrolysed by exogenous phospholipases. Secondly, PS may be brought to the membrane surface during membrane fusion accompanying the release reaction. Such a mechanism does, however, not explain the difference in procoagulant activity of platelets activated by thrombin or by collagen plus thrombin. Thirdly, the increased exposure of PS results from increased transbilayer movement of phospholipids (flipflop). This mechanism seems most likely, since it would also explain the decrease in SphM content of the outer leaflet of the plasma membrane. Rapid transbilayer movement of phospholipids may involve flipsites and may be dependent on cytoskeleton-membrane interaction, as dicussed in a previous section.

Treatment of platelets and erythrocytes with phospholipase $\mathrm{C}$ from $C$. welchii results in formation of diacylglycerol which becomes phosphorylated to PA at the cytosolic side of

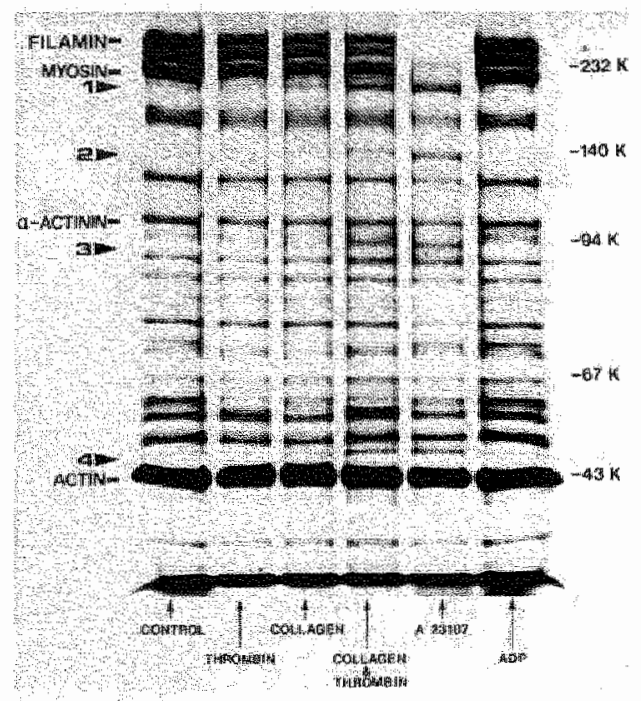

Fig. 8. Degradation of cytoskeletal proteins during stimulation of platelets by different procedures. Human platelets $\left(2.10^{7}\right.$ per $\left.\mathrm{ml}\right)$ were stimulated by $2 \mathrm{nM}$ thrombin, $10 \mu \mathrm{g} / \mathrm{ml}$ collagen, $1 \mu \mathrm{M}$ A23187, or $10 \mu \mathrm{M}$ ADP. The arrowheads at the left designate the four major degradation products (see also Fig.6). Indicared at the right are the positions of molecular weight standards in $\mathrm{kDa}$. Picture taken from [124]. the plasma membrane. During treatment with phospholipase C substantial amounts of PS appear at the platelet surface and platelets become procoagulant [125]. The amounts of PA formed are of the same order as formed by the action of endogenous phospholipase $C$ in activated platelets, suggesting that the amounts of diacylglycerol may produce non-bilayer structures by which enhanced flipflop can occur. It is emphasized that additional factors must play a role to explain why collagen and not thrombin enhances platelet procoagulant activity.

Involvement of the cytoskeletal proteins in the expression of platelet procoagulant activity is suggested by the results obtained with diamide. Treatment of platelets with diamide leads to extensive crosslinking of cytoskeletal proteins, particularly filamin, talín, myosin and actin [611]. In addition, PS becomes exposed and procoagulant activity increases [50]. More recently, examination of protein patterns obtained after gel electrophoresis showed that the prothrombinase activity of platelets activated by various agonists, correlated with the extent of proteolysis of cytoskeletal proteins by calpain (Fig. 8) [124]. In addition, platelets from a patient with a deficient generation of procoagulant activity displayed a diminished extent of degradation of cytoskeletal proteins [124]. These findings implicate cytoskeletal degradation by calpain to play a role in the expression of platelet procoagulant activity.

A model can be formulated which embodies both formation of non-bilayer structures and cytoskeletal degradation by calpain (Fig. 9). Formation of non-bilayer structures by diacylglycerol, as means of rapid transbilayer movement, is not sufficient to results in transport of phospholipids over the plasma membrane, regarding the inability of thrombin to render platelets procoagulant. Stimulation of calpain may be an additional prerequisite for flipflop of PS. It is conceivable that degradation of cytoskeletal proteins will cause alterations of cytoskeletal organization which may lead to a detachment of cytoskeleton from the plasma membrane. Assuming that platelet cytoskeletal proteins bind anionic phospholipids in the cytosolic leaflet of the plasma membrane, as is observed in erythrocytes, the detachment of the cytoskeleton from the plasma membrane would free the anionic lipids to participate in transbilayer movement along non-bilayer structures. 


\section{GLOSSARY OF THESIS CONTENTS}

At the start of the research period during which the experiments presented in this thesis were performed, the prevailing model for the loss of transbilayer asymmetry of PS during the expression of platelet procoagulant activity included both disruption of cytoskeleton-membrane interactions and formation of non-bilayer structures, as outlined above.

Evidence was already obtained showing that the extent of proteolysis of cytoskeletal proteins by calpain correlated with the extent of transbilayer movement of PS. Based on these observations, the correlation between calpain activity and platelet procoagulant activity was further investigated by comparing their $\mathrm{Ca}^{2+}$ dependence and their time-courses of generation after various platelet treatments. The results from these investigations are presented in chapter 2. In chapter 3 , the relation between calpain activity and phospholipid transbilayer movement in platelets is further demonstrated, making use of special properties of fluoridetreated platelets. Fluoride-treated platelets were also used to show that inhibition of platelet calpain leads to reduction of both cytoskeletal degradation and procoagulant activity. Additional evidence for the involvement of a decreased interaction between platelet cytoskeleton and plasma membrane in increased transbilayer movement of phospholipids came from experiments with artificially induced plasma membrane vesicles, as is shown in chapter 4.

Transbilayer movement of phospholipids in the platelet plasma membrane was investigated at the level of the lipid bilayer, employing the aspecific fluorescent membrane probe trimethylammonium-diphenylhexatriene (TMADPH). It was found that, besides a phospholipid transbilayer asymmetry, the platelet plasma membrane displays also a transmembrane fluidity gradient, as is described in chapter 5 .
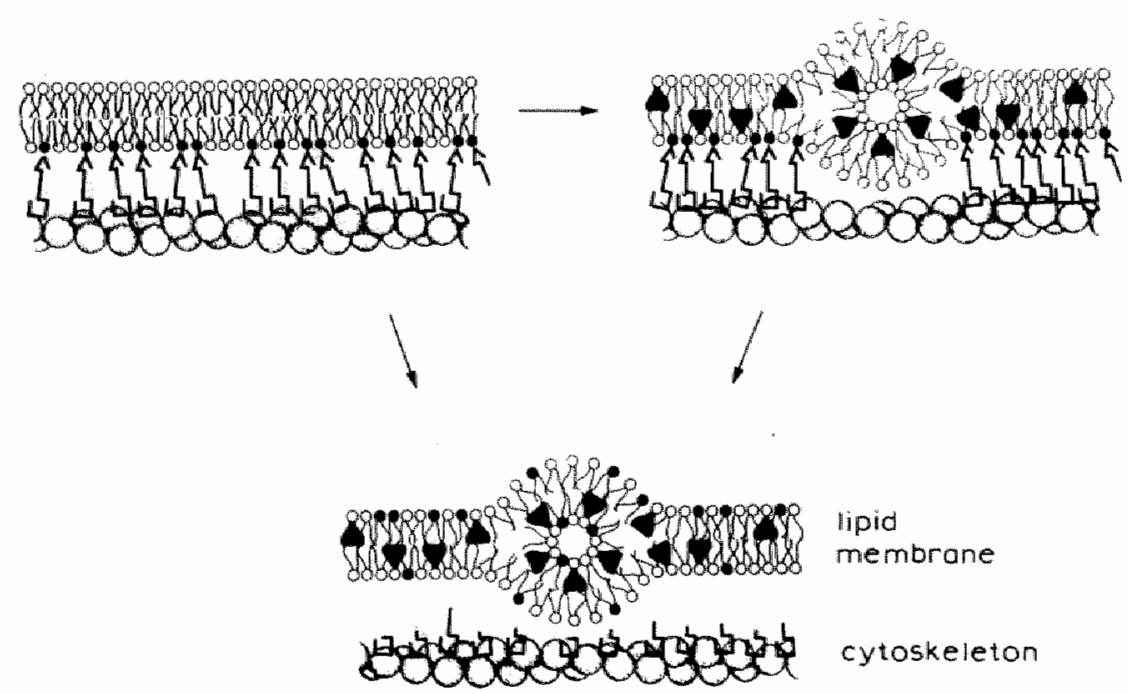

Fig. 9. Hypothetical model for the induction of phospholipid transbilayer movement in activated platelets. Phosphatidylserine (filled headgroups) is supposed to interact (arrows) with cytoskeletal proteins. Production of diacylglycerol (filled cones) will lead to formation of non-billayer structures (inverted micelle), along which rapid flipflop may accur. A prerequisite for PS to participate in rapid flipflop is breakage of its interaction with cytoskeletal proteins. 
Aspecific transbilayer movement of TMA-DPH enabled investigation of the occurrence of thipsites in the platelet plasma membrane during platelet activation. These investigations showed that activation-dependent flipflop of PS in the platelet plasma membrane is accompanied by the formation of flipsites, as is shown in chapter 6.
Finally, in the general discussion, the route of intracellular mechanisms leading to platelet procoagulant activity is outlined, and molecular mechanisms resulting in formation of flipsites and the role of the platelet membrane skeleton therein will be discussed. 


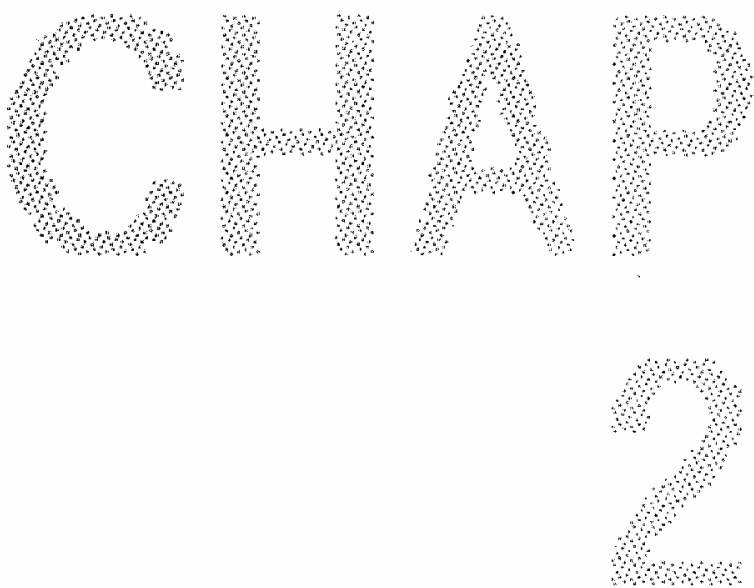




\title{
Platelet procoagulant activity and cytoskeletal degradation by calpain.
}

\begin{abstract}
The relationship between platelet calpain activity and platelet procoagulant activity was investigated by comparison of the time course of their generation after platelet stimulation by calcium ionophore $\mathbf{A 2 3 1 8 7}$, or by the combined action of collagen and thrombin, or during exposure of platelets to the local anesthetics dibucaine or tetracaine. In addition, the $\mathrm{Ca}^{2+}$ dose-response curves of both activities in intact platelets, obtained by stimulation with $\mathbf{A 2 3 1 8 7}$ in the presence of HEDTA $/ \mathrm{Ca}^{2+}$ buffers, were compared. Platelet procoagulant aetivity was determined by assaying for prothrombinase activity in the presence of saturating concentrations of factors $X_{a}, V_{a}$, and prothrombin. Platelet calpain activity was monitored by the degradation of its major substrates (filamin, talin, myosin) and the formation of their fragments as judged from protein patterns after gel electrophoresis. Platelet stimulation by $\mathbf{A 2 3 1 8 7}$ resulted in a fast increase in prothrombinase activity, reaching its maximum level after about 20 seconds. Filamin and talin were completely hydrolysed within 15 seconds, and myosin was partly degraded between 15 and 30 seconds after platelet activation. When platelets were activated by collagen plus thrombin, prothrombinase activity was: generated with a sigmoid time course, the steepest increase being observed between 1 and 2 minutes after platelet activation. Proteolysils of filamin and talim occurred between 0.5 and 1.5 minutes after platelet activation, while degradation of myosin became visible after 2 to 2.5 minutes. Dibucaine and tetracaine were both found to be potent stimulators of prothrombinase activity, with half maximal activities obtained at 0.7 and $2.8 \mathrm{mM}$ resp. Using suboptimal concentrations of both local anesthetics, it was found that the generation of prothrombinase activity closely parallelled that of calpain activity over a time course of 1 hour. $\mathrm{Ca}^{2+}$ titration of intact platelets using $\mathrm{A23187}$ and $\mathrm{Ca}^{2+} / \mathrm{HEDTA}$ buffers, revealed half maximal response at about $15 \mu \mathrm{M}$ free $\mathrm{Ca}^{2+}$ for both calpain and prothrombinase activity. These findings strongly suggest a causal relationship between generation of a procoagulant platelet surface and calpain-mediated degradation of flamin, talin, and myosin. Since an increased procoagulant activity reflects an increased exposure of phosphatidylserine at the platelet outer surface, the present findings suggest that platelet cytoskeletal proteins are involved in the regulation of membrane lipid asymmetry.
\end{abstract}




\section{INTRODUCTION}

One of the responses that platelets display upon activation is the generation of a procoagulant surface at the outside of the plasma membrane $[54,543,730,735]$. This procoagulant surface greatly enhances the rate of two important sequential reactions in the coagulation cascade: the activation of factor $\mathrm{X}$ by a complex of factor IXa and factor VIIIa, and the activation of prothrombin by a complex of factor $\mathrm{Xa}_{\mathrm{a}}$ and factor $\mathrm{Va}$ (the prothrombinase complex) [543]. The major determinant of platelet procoagulant activity is the membrane phospholipid phosphatidylserine, which upon activation becomes exposed at the outer surface of the platelet plasma membrane as a result of a transbilayer movement of phospholipids [49, $50,543]$

The agonist pattern of platelet procoagulant activity [50] is different from any other platelet response: collagen is the only single physiological agonist able to significantly increase platelet procoagulant activity. Higher stimulation of platelet procoagulant activity is achieved by the combined action of collagen and thrombin. The most potent stimulator of platelet procoagulant activity, however, is the $\mathrm{Ca}^{2+}$ ionophore A23187, indicative for the importance of increased cytosolic $\mathrm{Ca}^{2+}$ levels in this response. Investigation of the protein pattern from platelets activated by different agonists showed a strong correlation between the extent of expression of platelet procoagulant activity and the extent of proteolysis of cytoskeletal proteins by calpain $\uparrow[124]$.

In view of findings that interactions between membrane skeletal proteins and anionic phospholipids may stabilize membrane lipid asymmeiry in erythrocytes $[168,217,256,423$, 572], it has been suggested that calpain-mediated degradation of cytoskeletal proteins may be a prerequisite for the loss of membrane lipid

\footnotetext{
* The name calpain (E.C.3.4.22.17) is used for the ut biquitous $\mathrm{Ca}^{2+}$-dependent neutral thiol-protease [431] originally described in platelets by Phillips and Jakabowa 4499]. The platelet protein known as P235 $\left(\mathrm{M}_{\mathrm{r}} 235000\right)$ is named talin, in accordance with the demonstrated similarity between $\mathrm{P} 235$ and talin $[470,31]$. The name filamin [6B3] is used for ABP (platelet actin-binding-protein, $\mathrm{Mr}_{\mathrm{r}}$ 250000), considlering their identical properties [212] and immunochemical cross-reactivity [472].
}

asymmetry which occurs during the expression of platelet procoagulant activity [124].

In order to substantiate this suggestion, the correlation between stimulation of calpain activity and stimulation of platelet procoagulant activity was further explored by investigation of their time-response relationship after platelet activation by collagen plus thrombin, by A23187, and by exposure of platelets to the local anesthetics dibucaine or tetracaine, which have been reported to activate platelet calpain $[119,442,608]$. Furthermore, the $\mathrm{Ca}^{2+}$ response relationship of both actiwities was investigated, using $\mathrm{Ca}^{2+}$ buffers and $\mathrm{A} 23187$ to equilibrate extra- and intra-cellular free $\mathrm{Ca}^{2+}$.

\section{MATERIALS AND METHODS}

Blood coagulation factors $\mathrm{Va}, \mathrm{Xa}$, prothrombin and thrombin were isolated and purified according to Rosing et.al. [544]. Collagen type I from equine tendon was obtained from Hormon Chemie (München, Germany). The thrombin-specific chromogenic substrate, \$2238 (H-Dphenylalanyl-L-pipecolyl-L-arginine $6 \mathrm{p}$-mitroanilide dihydrochloride), was purchased from Kabi Diagnostica (Stockholm, Sweden). Dibucaine- $\mathrm{HCl}$ and tetracaineHCl were from ICN Pharmaceuticals (New York, USA) and from Sigma (St. Louis, USA) resp. Ionophore A23187 was obtained from Calbiochem-Hoechst (San Diego, USA). HEDTA (N-hydroxyethylethylenc-diaminetriacetic acid) was purchased from Sigma (St. Louis, USA) Other (bio)chemicals were of the highest grade commercially available.

\section{Platelet isolation}

Human platelets were isolated from freshly drawn blood as described previously [51]. Washed platelets were resuspended in a calcium-free buffer at $\mathrm{pH} 7.4$, containing $10 \mathrm{mM}$ Hepes (4-(2-hydroxyethyl)-1-piperazimeethanesulfonic acid), $136 \mathrm{mM} \mathrm{NaCl}, 27 \mathrm{mM} \mathrm{KCl}, 2 \mathrm{mM}$ $\mathrm{MgCl}_{2}, 5 \mathrm{mM}$ glucose and $0.1 \%$ fatty acid free humam serum albumin (Hepes buffer). Platelet concentration was adjusted to approximately $10^{8} \mathrm{ml}^{-1}$, using a Coulter Counter. Platelet lysis was determined by measuring lactate dehydrogenase activity in a $12000 \mathrm{xg}$ supernatant according to Wroblewski and La Due [710].

\section{Assay of platelet procoagulant activity}

Platelet procoagulant activity was determined essen. tially as described by Rosing et.al. [543], by assaying for prothronibinase activity under conditions at which the procoagulant surface is rate limiting. The assay was carried out at $37^{\circ} \mathrm{C}$ in Hepes buffer under continuous stirring (300 rev/min). To monitor the generation of prothrombinase activity after stimulation by collagen plus thrombin or A23187, factor $\mathrm{Xa}$, factor $\mathrm{Va}$, and $\mathrm{CaCl}_{2}$ were added to prewarmed Hepes buffer 45 seconds before the start of 
the assay. 15 Seconds later A23187 or collagen phus thrombin were added. Prothrombin was added 15 seconds before the assay was started by addition of platelets. Final

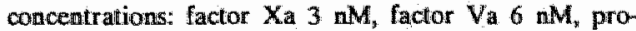
thrombin $4 \mathrm{mM}, \mathrm{CaC2} 3 \mathrm{mM}, \mathrm{A} 231871 \mu \mathrm{M}$, collagen 10 $\mu \mathrm{g} / \mathrm{ml}$, thrombin $4 \mathrm{nM}$, platelets $510^{6} \mathrm{ml}^{7}$. After various time infervals, the activation of prothrombin was arrested by diluting a $25 \mu$ aliquot of the assay mixture into a cuvette containing $1 \mathrm{ml}$ buffer composed of $50 \mathrm{~mm}$ Tris $/ \mathrm{HCl}, 100 \mathrm{mM} \mathrm{NaCl}, 2 \mathrm{mM}$ EDTA, pH 7.5. Thrombiti actruvity was determined spectrophotometrically at $405 \mathrm{~nm}$, after addition of chromogenic substrate $\$ 2238$ to a final concentration of $150 \mu \mathrm{M}$. The amount of thrombim formed was calculated from the change in absorbance per minute, using a calibration curve made with active-site titrated thrombin.

To monitor the time-dependent effect of dibucaine and tetracaine on platelet procoagulant activity, platelets were incubated with local anesthetics at a count of $110^{8}$ $\mathrm{mil}^{-1}$. After various time intervals a subsample was as. sayed for prothrombinase activity after dilution to $510^{6}$ platelets $\mathrm{ml}^{-1}$. Thrombin formation was measured one minute after the addition of the subsample to the assay mixture containing factor $\mathrm{Xa}$, factor $\mathrm{Va}$, prothrombin, and $\mathrm{CaCl}_{2}$ in Hepes buffer, as described for collagen plus thrombin and A.23187.

\section{$\mathrm{Ca}^{2+}$ titration of prothrombinase activity}

All $\mathrm{Ca}^{2 *}$ litrations were carried out at $37^{\circ} \mathrm{C}$ in a magnesium-free HEDTA/Ca ${ }^{2+}$ buffer $(10 \mathrm{mM}$ Hepes, $120 \mathrm{mM} \mathrm{NaCl}, 3 \mathrm{mM} \mathrm{KCl}, 5 \mathrm{mM}$ glucose, $30 \mathrm{mM}$ HEDTA, various amounts of $\mathrm{CaCl}_{2}, \mathrm{pH} 7,4$ ), at a platelet count of $2.10^{8} \mathrm{ml}^{-1}$ and under continows stirning An apparent $\mathrm{Kd}$ for $\mathrm{HEDTA} / \mathrm{Ca}^{2+}$ of $1.9 \mu \mathrm{M}$ was determined under the same conditions as used for the $\mathrm{Ca}^{2+}$ titrations by computer fitting of the titration curve, obtained when a $\mathrm{Ca}^{2+}$ solution of known titer was titrated with a HEDTA solution, using a $\mathrm{Ca}^{2+}$-selective ellectrode and a recording automatic titrator (Radiometer, Copenhagen, Sweden; type F2110Ca and RTS-system resp.). The free $\mathrm{Ca}^{2+}$ concentration of the various $\mathrm{Ca}^{2+}$ buffers was aleulated using the apparent $K a$ for $H E D T A C a^{2+}$, and was verified with the $\mathrm{Ca}^{2+}$-selective electrode. It was found that the $\mathrm{Ca}^{2}$ buffering capacity of the employed HEDTAVCa ${ }^{2}$ buffer was sufficient to mainitain applied extracellular free $\mathrm{Ca}^{2+}$ concentrations when platelets were challenged with A23187, over the wholle range of extracellular free $\mathrm{Ca}^{2+}$.

The $\mathrm{Ca}^{2+}$ dose-response relationship of platelet procoagulant activity was determined as follows: one minute after addition of 1 wol platelets (suspended in $50 \mathrm{mM}$ Hepes buffer pH 7.4 at $210^{9} \mathrm{ml}^{\mathrm{l}}$ ) to 9 vol. HEDTAVCa ${ }^{2+}$ buffer, A23187 was added from a stock sollution in DMSO to a final concentration of $0.3 \mu \mathrm{M}$ (final DMSO concentration: $0.5 \%$ ). Platelets were left to equilibrate with extracellular free $\mathrm{Ca}^{2+}$ for 5 minute at $37{ }^{\circ} \mathrm{C}$ under contunuous stirring. Then formaldehyde was added to a final concentration of $300 \mathrm{mM}$. After 5 minutes a sample was taken and diluted into Hepes buffer containing $1 \%$ fatty acid free human serum albumin and $3 \mathrm{mM}$ $\mathrm{Ca}^{2+}$ lo a platelet count of $5.10^{6} \mathrm{ml}^{-1}$. Prothrombinase activity was assayed by determination of the amount of thrombin formed in one mimute, after the addition of fat tor $\mathrm{Xa}$, factor $\mathrm{Wa}$, and prothrombin.

The fuxation of platelets after equilibration with the $\mathrm{Ca}^{2+}$ buffer is necessary to prewent post-activathmin ot procoagulant act ivity by $\mathrm{A} 23187$-nediated antry of Cat" which has to be present at millimolar concentrations dur" ing the prothrombinase assay for functional binding of the prothrombinase complex to a procoagulant surface. Hit wiss found that residual formaldehyde, present during the prothrombinase assay, inhibited prothrombinase activity by some 50\%. "This inhibition howewer $_{*}$ was independent of the extemit of prothrombinase activity itsedit, and independent of the free $\mathrm{Ca}^{2+}$ concentration of the $\mathrm{Ca}^{2+}$ buf fer.

\section{Detemination of calpain activity by gel electro- phoresis.}

For proper correlation of calpain actiwity with prothrombinase activity, the same batch of platelets was used for both determinations throughout all experintents. Generation of calpain activity after platelet stimulation by A231.87 or collagen plus thrombin was monitored at a higher platelet covint than used for the prothrombinase assay, for practical reasons. The protein patterns were found not to be affected by the increased platefet count.

At $37 \%$ under continuous stirring washed humain plattelets were added to human serum albumin-free Hepes buffer containing $3 \mathrm{mM} \mathrm{CaCl}$ and the stimulators. After various time intervals calpain activity was arrested by addition of an equal volume of Hepes buffer, $\mathrm{pH} 6.6$, containing $2 \mathrm{mM}$ mersalylic acid, $2 \mathrm{mM}$ phenyllmethane sulphonylluoride, $2 \mathrm{mM}$ benzanidine and $20 \mathrm{mM}$ EGTA. After collection of platelets by centrifugation at $5000 \mathrm{mxg}$ for 5 minutes in the presence of $15 \%$ acid-citrate-dexirose (180 mM glucose, $80 \mathrm{mM}$ trisodium citrate, $52 \mathrm{mM}$ citric acid), gell samples were prepared by dissolving the platelet pellet to a concentration of $2.10^{9} \mathrm{ml}^{-4}$ in sample buffier (62.5 mM Tris $/ \mathrm{HCl}, 2 \%$ SDS, $10 \%$ glycerol, $3 \%$ mercaptoethanol, .005\% broomphenolblus, $10 \mathrm{mM}$ EOTA, $1 \mathrm{mM}$ phenylmethanesulphonylchloride; $1 \mathrm{mM}$ benzanidine, $1 \mathrm{mM}$ mersalilyc acid), Routinely all samples were bolled for 5 munutes before application to the gel. Disc electrophoresis was performed acording to Laemmli [356], using $6-12 \%$ gradient polyacrylamide allab gels with is $3 \%$ polyacrylamide stacking gel:

Generation of calpain acivity during incubation of platelets $\left(10^{8} \mathrm{~m}^{-1}\right.$ in Hepes buffer) with dibucaline or te tracaine, was followed by arresting and processing sulbsamples taken after various time interwals, as described for A23187 and collagen plus thrombin.

The offect of intracellular free $\mathrm{Ca}^{2+}$ concentration on platelet calpain activity was determined by equilibration of wastied platelets with various $H E D T A / C{ }^{2+}$ buffers in the presence of $0.3 \mu \mathrm{M}$ A23187, as described for this determination of prothrombinase activity. After 10 minutes plateletw were arrested and procesced as described for collagen plus thrombin. 


\section{RESULTS}

\section{Generation of calpain- and prothrombinase- activity by $\mathrm{A} 23187$.}

The $\mathrm{Ca}^{2+4}$ ionophore $\mathrm{A} 23187$, in the presence of extracellular $\mathrm{Ca}^{2+}$, is a very potent stimulator of platelet procoagulant activity: within one minute full activity is already expressed [530]. In order to monitor the generation of prothrombinase activity on such a short time scale, platelets were activated in the presence of all components of the prothrombinase complex, and samples were taken every 5 seconds to determine the amount of thrombin formed. In this way a time course of thrombin formation was obtained which initially increases exponentially, and then gradually develops into a straight line (Fig. 1A), reflecting the generation of prothrombinase activity to a constant level. Since this curve represents the time course of thrombin formation, the actual rate of thrombin formation (prothrombinase activity) at any time is reflected by the tangent of the curve at that time point. In this way the data were recalculated as prothrombinase activity relative to the final level (Fig. 1A). It was found that upon activation of platelets with A23187, prothrombinase activity is increased without any obvious delay and almost in a linear fashion, to reach its maximum level after about 20 seconids.

A complicating factor in the determination of prothrombinase activity on such short time scalles, is the non-zero assembly time needed. for the components of the prothrombinase complex to form a functional unit on the procoagulant surface. To overcome this problem, high concentrations of factor $\mathrm{Xa}$ and factor $\mathrm{Va}$ were applied ( 15 and $30 \mathrm{nM}$ resp.). It was found that under these conditions the assembly time was negligible.

Platelet calpain activity was monitored by the degradation of its major substrates and the formation of their fragments as judged from protein patterns obtained after polyacrylamide gel electrophoresis of reduced protein samples. The major substrates of calpain are the cytoskeletal proteins filamin, talin, and myosin $[124,216,499,608,656,690]$. The major degradation products are found at $190 \mathrm{kDa}$ (fragment 1, derived from filamin [212, 648] and talin $[31,212,292,470]$ ), at $135 \mathrm{kDa}$ (fragment 2 , originating from myosin $[124,296$, 492]), at $93 \mathrm{kDa}$ (fragment 3 , coming from filamin $[212,648]$ ), and at $48 \mathrm{kDa}$ (fragment 4 , produced from talin $[31,212,292,470])$. When platelets are activated by $\mathrm{A} 23187$ in the presence of $3 \mathrm{mM}$ extracellular $\mathrm{Ca}^{2+}$, calpain activity is already evident after about $10 \mathrm{sec}-$ onds (Fig. 1B). Filamin and talin are most
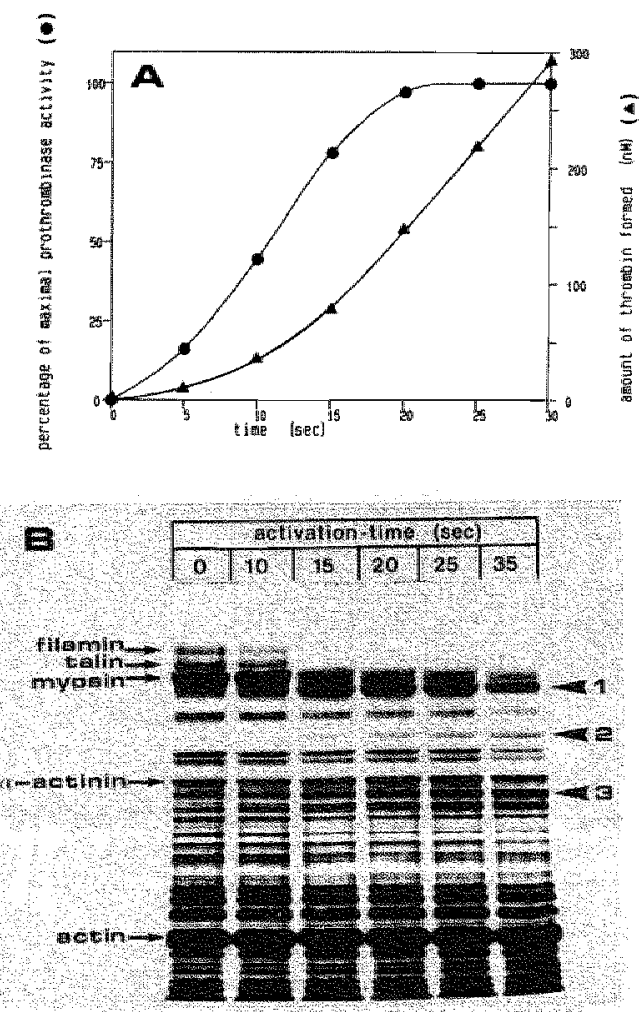

Fig. 1. Generation of prothrombinase activity (A) and calpain activity (B) by A23187. (A): Gen eration of prothrombinase activity (@) was reconstructed from the time course of thrombin formation $(\boldsymbol{\Lambda})$ by determination of the slope of its tangent at different time points. Prothrombinase activity was assayed as described in the methods, with the exoeption that factor $\mathrm{Xa}$ and factor Va were present at resp. 15 and $30 \mathrm{nM}$. (B): Washed human platelets, at $110^{8} \mathrm{ml}^{-1}$, were stimulated by $1 \mu \mathrm{M}$ A23187. Calpain activity is monitored after gel electrophoresis by the appearance of fragment 1 (firom filamin and talin), fragment 2 (from myosin), fragment 3 (from Cilamin), and fragment 4 (from talin). The fragments are indicated at the right by numbered arrows. At the left the major cytoskeletal proteins are identified. The data shown are taken from a representative experiment. 
rapidly degraded the next 5 seconds, followed by proteolysis of myosin.

When the generation of platelet procoagulant activity and calpain activity after stimulation by $\mathrm{A} 23187$ are compared, it is obvious that filamin and talin are completely degraded when half maximal prothrombinase activity is reached, while myosin degradation becomes apparent at maximal prothrombinase activity.

\section{Generation of calpain-and prothrombinase- activity by the combined action of collagen plus thrombin.}

Upon stimulation of washed platelets by both collagen and thrombin in the presence of $\mathrm{Ca}^{2+}$ and the factors of the prothrombinase complex, platelet procoagulant activity developed slowly during the first minute, followed by a steep increase between 1 and 2 minutes
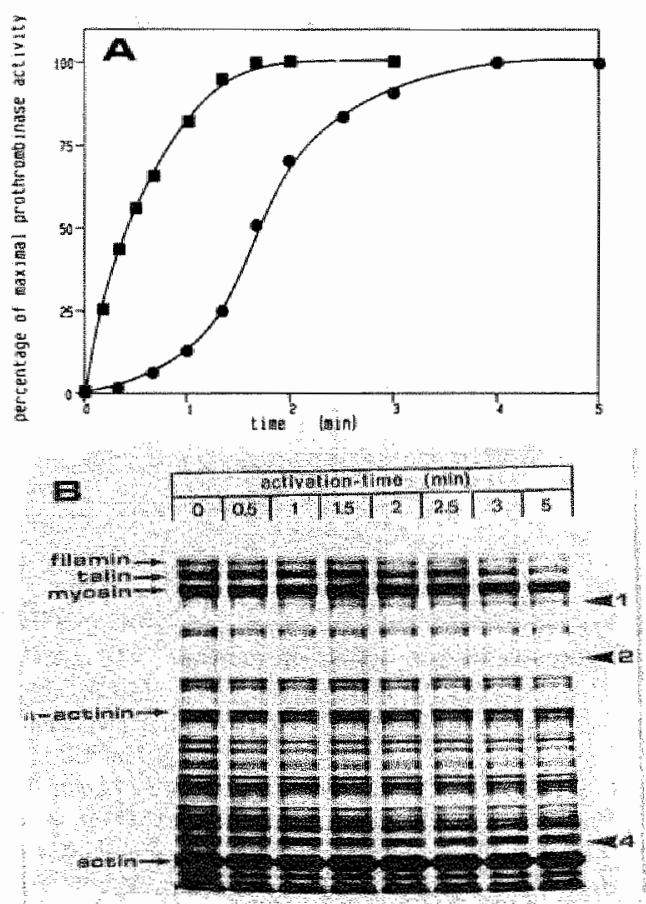

after platelet stimulation, and gradually levell ing off after 2 to 4 minutes (Fig. 2A). At this time scale the assembly time of the prothrombinase complex was not rate limiting.

Development of calpain activity was judged by the appearance of fragment 1 and fragment 2 (Fig. 2B). Most of fragment 1 wwas produced berween 0.5 and 1.5 minutes after platelet stimulation, while fragment 2 appeared only faintly at about 2 minutes. Clearly, the degradation of filamin and talin by calpain coincides with the initial phase of generation of prothrombinase activity when platelets are stimulated by colliagen plus thrombin.

Previously we have shown that when platelets are challenged with collagen plus thrombin in the presence of EDTA, proteolysis of cytoskeletal proteins by calpain does not occur [124]. Based on this observation we investigated the generation of both calpain- and procoagulant-activity after the addition of millimolar $\mathrm{Ca}^{2+}$ to platelets incubated with collagen plus thrombin for 5 minutes in the presence of 1 mM EGTA (Fig. 2A). It was found that immediately after the addition of $\mathrm{Ca}^{2+}$, the prothrombinase activity steeply increased to its maximum level in 1.5 minute, while partial proteolysis of filamin and tallin ocurred during the first 20 seconds after the addition of $\mathrm{Ca}^{2}$ (Fig. 2C).

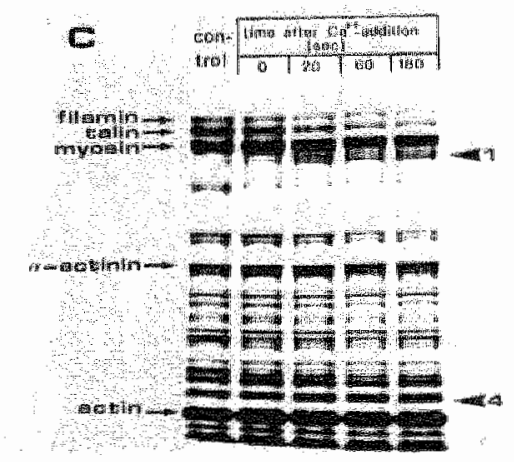

Fig. 2. Generation of prothrombinase activity and calpain activity by the combined action of collagen plus thrombin. Dewelopment of prothrombinase activity and calpain activity was followed after platelet activation by collagen $(10 \mu \mathrm{g} / \mathrm{ml})$ plus thrombin $(4 \mathrm{nM})$, either in the presence of $3 \mathrm{mMCa}{ }^{2+}(A, ; B$ resp), or after addition of $4 \mathrm{mM} \mathrm{Ca}^{2+}$ to platelets previously activated with collagen plus thrombin for 5 minutes in the presence of $1 \mathrm{mM}$ EGTA $(A,-C$ resp.). The lane identified by control $(C)$ refers to untreated platelets. Incubations for gell electrophoresis were performed at a platelet count of $510^{7} \mathrm{mr}^{1}$. Reference arrows aside the protein patterns are explained in Fig. 1. The data shown are taken from a representative experiment. Experimental details are outlined in the methods. 
Generation of calpain- and prothrombinaseactivity by local amesthetics.

To further explore the relationship between platelet calpain and procoagulant activity the local anesthetics dibucaine and tetracaine were employed. These agents seemed especially attractive, since they were reported to activate calpain $[119,442,608]$, while they inhibit platelet aggregation and secretion [197]. First, their ability to generate platelet procoagulant activity was investigated at various concentrations. It was found that both dibucaine and tetracaine were potent stimulators of prothrombinase activity (Fig. 3). Dibucaine was 4 to 5 times more potent than tetracaine. Above $1 \mathrm{mM}$ dibucaine or $3.5 \mathrm{mM}$ tetracaine, measurements became unreliable as a result of considerable platelet lysis. Below these concentrations no significant lysis occurred.

To compare the generation of calpain- and procoagulant-activity by the local anesthetics, platelets were incubated with a suboptimal dose of dibucaine or tetracaine. In the presence of $0.5 \mathrm{mM}$ dibucaine, platelet prothrombinconverting activity was raised to a maximum level within 45 minutes, with most of the increase after the first 10 minutes (Fig. 4A). When samples, taken from the same incubation, were investigated for calpain activity

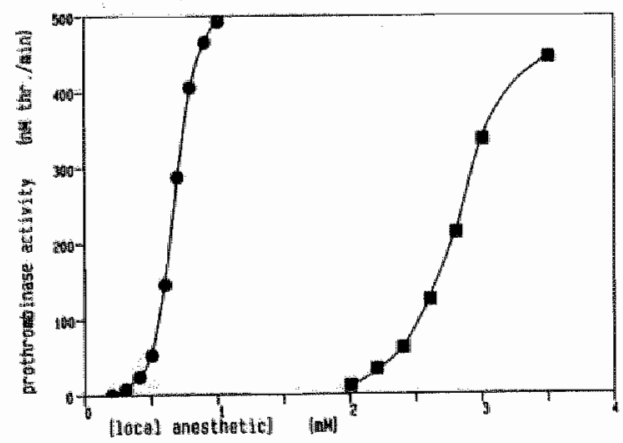

Fig. 3. Dose-dependent stimulation of prothrombinase activity by the local anesthetics dibucaine $(O)$ or tetracaine $(a)$. Platelets $\left(510^{6}\right.$ $\left.\mathrm{mil}^{\mathrm{l}}\right)$ ) were challenged with the local anesthetics for 10 minutes before prothrombinase activity was determined one minute after addition of the components of the prothrombinase complex, as described in the methods. The data shown are obtained from a representative experiment.
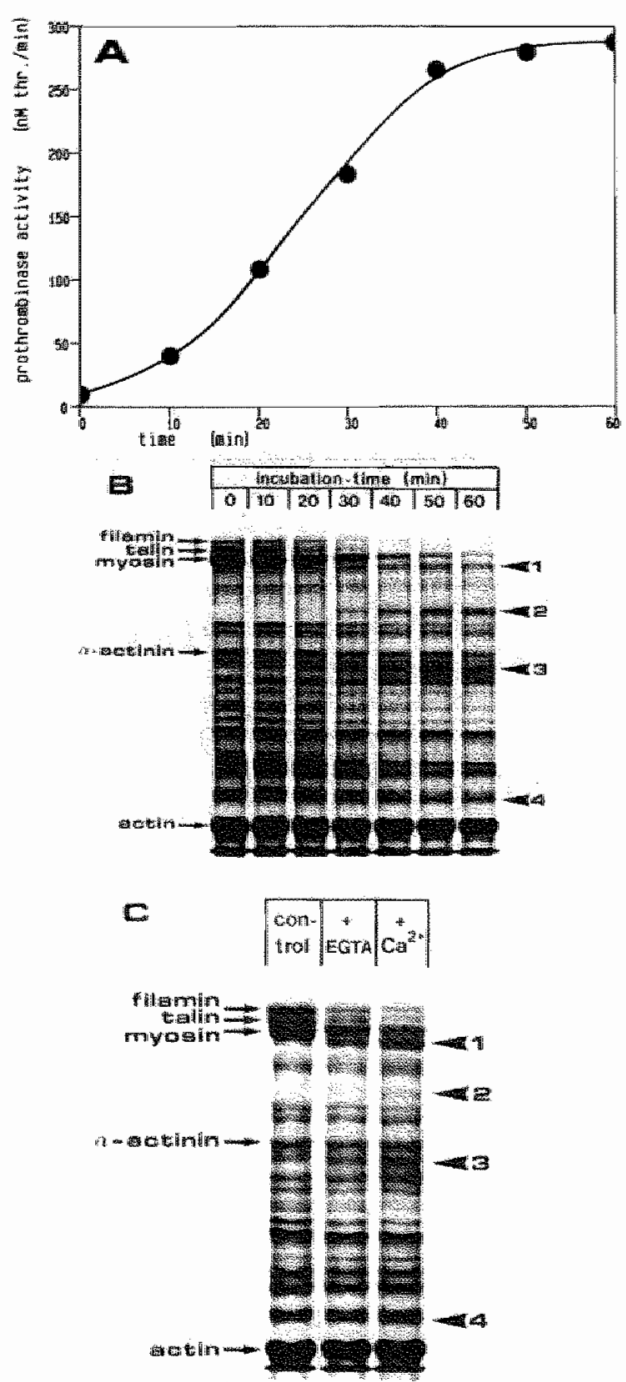

Fug. 4. Generation of prothrombinase activity and calpain activity by dibucaine. The effect of dibucaine $(0.5 \mathrm{mM})$ in the presence of $3 \mathrm{mM} \mathrm{Ca}^{2+}$ on the development of prothrombinase activity and calpain activ. ity is shown in parel $A$ and panel $B$ resp. Panel $C$ shows the effect of $1 \mathrm{mM}$ EGTA on the stimulation of calpain by $1 \mathrm{mM}$ dibucaine for 10 minutes (lane: + EGTA), in comparison with a similar stimulation in the presence of $1 \mathrm{mM}$ $\mathrm{Ca}^{2+}$ (lane: $\left.+\mathrm{Ca}^{2+}\right)$, and in comparison with untreated platelets (control lane). The data shown are taken from a representative tativeexperiment. A: Prothrombinase activity was assayed after dilution to $510^{6}$ platelets $\mathrm{ml}^{-1}$, as described in the methods. Untreated platelets retained a constant basal level of prothrombinase activity throughout the incubation period. $B_{n} C:$ reference arrows aside the protein patterns are explained in Fig. 1. Samples for gel electrophoresis were prepared as outlined in the methods. 
(Fig. 4B), it was found that between 10 and 50 minutes after the addition of dibucaine, cytoskeletal degradation develops to its maximum level. This time course coincides with that of the generation of platelet procoagulant activity by dibucaine. When platelets were incubated with $2 \mathrm{mM}$ tetracaine, a similar correlation as with dibucaine was observed between the generation of calpain activity and prothrombimase activity, on a time scalle of one hour (not shown).

Incubation of washed platelets with $1 \mathrm{mM}$ dibucaine for 10 minutes in the presence of 1 . mM EGTA did not result in inhibition of prothrombinase activity (not shown), while cytoskeletal degradation by calpain was only moderately inhibited, in comparison to a similar incubation in the presence of $1 \mathrm{mM} \mathrm{Ca}^{2+}$ (Fig. 4C). When platelets were lysed by sonication in the presence of EGTA, subsequent addition of $1 \mathrm{mM}$ dibucaine did not produce any degradation of cytoskeletal proteins (not shown). Therefore, activation of platelet calpain by local anesthetics is not dependent on extracellular $\mathrm{Ca}^{2+}$, and is not a result of direct interaction with calpain.
$\mathrm{Ca}^{2+}$ dose-response relationship of the stimulation of calpain and procoagulant activity.

To gain additional support for a causal relationship between calpain- and procoagulantactivity the response to the level of intracellular free $\mathrm{Ca}^{2+}$ of both activities was examined by stimulation of washed platelets with $\mathrm{A} 23187$ $(0.3 \mu \mathrm{M})$, in the presence of HEDTAVCa ${ }^{2+}$ buffers at various free $\mathrm{Ca}^{2+}$ levels.

The dependence on free $\mathrm{Ca}^{2 *}$ of the generation of platelet procoagulant activity, is shown in Fig. 5A. It was found that prothrom-

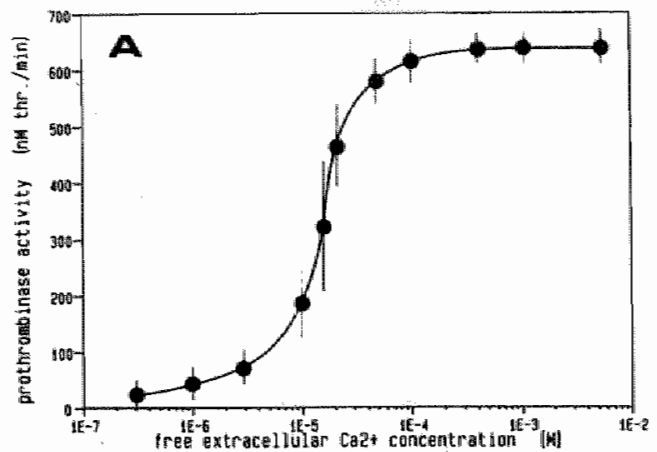

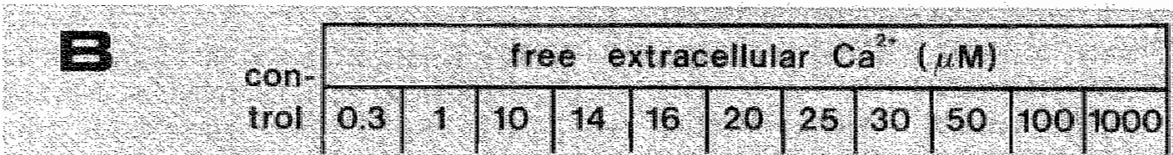

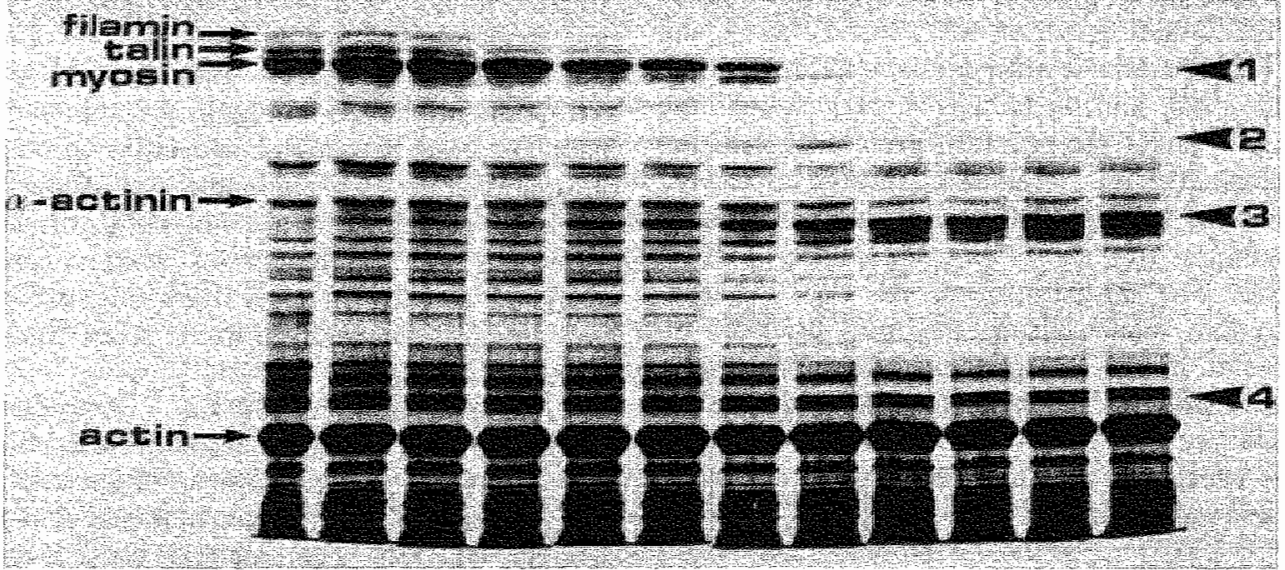

Fig. 5. $\mathrm{Ca}^{2+}$ dose-response relationship of platelet prothrombinase activity (A) and calpain activity (B). Washed human platelets, at a concentration of $210^{8} \mathrm{ml}^{-1}$, were challenged with $0.3{ }_{12} \mathrm{M}$ A23187 in the presence of $30 \mathrm{mM}$ HEDTA $/ \mathrm{Ca}^{2+}$ buffer. Prothrombinase activity was determined after fixation with formalldehyde and dillution to $510^{6}$ platelets.ml ${ }^{-1}$. Protein samples were prepared after arrest of calpain activity by addition of surplus EGTA and protease inhibitors. The data shown in $(A)$ are the mean of the 3 experiments. The data shown in (B) are taken from a representative experiment. Experimental details are described in the methods. 
binase activity increased slowly upon raising free $\mathrm{Ca}^{2+}$ from submicromolar levels. Between 5 and $30, \mathrm{M}$ free $\mathrm{Ca}^{2+}$, prothrombinase activity deweloped with a steep increase. Half-maximal activity was obtained at $15 \mu \mathrm{M}$ free $\mathrm{Ca}^{2+}$. Beyond $30 \mu \mathrm{M}$ free $\mathrm{Ca}^{2+}$, prothrombinase activity gradually leveled off to reach maximall activity at $100 \mu \mathrm{M}$ free $\mathrm{Ca}^{2+}$.

Investigation of calpain activity over the same range of free extracellular $\mathrm{Ca}^{2+}$ levels (Fig. SB), led to the following observations: (i) Hydrolysis of filamin and talin already starts at submicromolar levels of free $\mathrm{Ca}^{2+}$ as evidenced by the appearance of fragment(s) 1 and fragment 3. Major degradation of cytoskeletal proteins takes place going from 10 to $20 \mu \mathrm{M}$ free $\mathrm{Ca}^{2+}$, and between 20 to $25 \mu \mathrm{M}$ free $\mathrm{Ca}^{2+}$ all filamin and talin are degraded. (ii) Hydrolysis of heavy-chain myosin rapidly increases just above $10 \mu \mathrm{M}$ free $\mathrm{Ca}^{2+}$, as judged from the appearance of fragment 2. Between 20 to 30 $\mu \mathrm{M}$ free $\mathrm{Ca}^{2+}$ myosin degradation is completed. (iii) In addition to the changes in the protein pattern with regard to the major cytoskeletal proteinsfilamin, talin, and myosin, several other proteins are affected by $\mathrm{Ca}^{2+}$-dependent proteolysis. Noteworthy is the decrease in intensity of a protein band corresponding to the position of aractinin (105 kDa), especially when free $\mathrm{Ca}^{2+}$ is raised above a level of $20 \mu \mathrm{M}$. Other $\mathrm{Ca}^{2+}$-dependent changes in the protein pattern involving unidentified proteins are decreased bands corresponding to $170,160,130,83,75,69$, and $56 \mathrm{kDa}$, and new protein fragments at 140,135 , 125,120 , and $90 \mathrm{kDa}$ (Fig. 5B). At all free $\mathrm{Ca}^{2+}$ levels the extent of protein degradation obtained after 15 minutes exposure to A23187 did not differ from those obtained after 5 minutes treatment with lionophore. Platelet lysis was insignificant at all $\mathrm{Ca}^{2+}$ concentrations. Generalizing, the most dramatic increase in $\mathrm{Ca}^{2+}$-dependent proteolysis of platelet proteins is observed between 15 and 25 $\mu \mathrm{M}$ free $\mathrm{Ca}^{2+}$. This level is quite similar to that at which half maximal activity of prothrombinase is observed.

\section{DISCUSSION}

The experiments presented in this study demonstrate that platelet calpain- and procoagulant-activity are closely related phenomena with regard to the time course of their development after stimulation of platelets with A23187 or collagen plus thrombin, or after exposure of platelets to the local anesthetics dibucaine or tetracaine. This parallel generation covers a time scale ranging from less than half a minute to one hour. The similarity between the $\mathrm{Ca}^{2+}$-dependence of prothrombinase activity and calpain activity further strengthens their relationship.

The local anesthetics dibucaine and tetracaine appeared to be convenient agents to investigate the relationship between platelet procoagulant activity and calpain activity, since they were already known to stimulate calpain $[119,442,608]$. We found that local anesthetics are also able to stimulate platelet prothrombinase activity, supporting a finding by Peerschke [490] who used a clotting assay. The simultaneous stimulation of platelet prothrombinase activity and calpain activity by the local anesthetics strongly supports the notion that these two platelet processes are causally related.

It is generally observed that local anesthetics are inhibitors of other platelet activation phenomena [10, 197, 240, 377, 725]. More specifically, it has been shown that they inhibit phospholipase A2 $[196,660]$ and protein kinase C $[274,659]$. Therefore, the use of local anesthetics implicates that many processes normally involved in platelet activation (protein phosphorylation, arachidonate metabolism, secretion, aggregation) are not obligatory involved in the expression of platelet procoagulant activity. In fact, these implications have already been deduced from earlier experiments $[48,734]$.

The potent effect of $\mathrm{Ca}^{2+}$ ionophore A23187 both on calpain- and on prothrombinase-activity, suggests that uptake of extracellular $\mathrm{Ca}^{2+}$ may be required for both activities to develop. The role of extracellular $\mathrm{Ca}^{2+}$ is also obvious from the difference between the time course of development of prothrombinase activity of platelets triggered by collagen plus thrombin in the presence of $\mathrm{Ca}^{2+}$, and of platelets to which $\mathrm{Ca}^{2+}$ was added after previous 
activation by collagen plus thrombin in the presence of EGTA. In the second situation prothrombinase activity steeply increased immediately after the addition of $\mathrm{Ca}^{2+}$, while in the first situation such a steep increase is only observed after a lag phase of about 1 mimute. From these observations it is concluded that the entry of extracellular $\mathrm{Ca}^{2+}$ is an obligatory step in the generation of platelet procoagulant activity by collagen plus thrombin, and that the influx of this extracellular $\mathrm{Ca}^{2+}$ is enabled only after a delay of approximately 1 minute.

In contrast, the stimulatory effect of local anesthetics on calpain activity was independent of the presence of extracellular EGTA and was not a result of direct activation of calpain. Therefore, $\mathrm{Ca}^{2+}$ must have been released from intracellular sources, though influx of extracellular $\mathrm{Ca}^{2+}$, if present, may also occur [490]. Most likely, this intracellular source is membrane-bound $\mathrm{Ca}^{2+}$, or more specifically lipidbound $\mathrm{Ca}^{2+}$ being displaced by dibucaine or tetracaine $[142,315,536]$.

Quantitative information about the levels of cytosolic free $\mathrm{Ca}^{2+}$ can be obtained from the $\mathrm{Ca}^{2+}$ titrations of platelet calpain- and procoagulant-activity, which both display the major increase at 10 to $15 \mu \mathrm{M}$ free extracellular $\mathrm{Ca}^{2+}$. The reliability of this information is based on the assumption that $0.3 \mu \mathrm{M} \mathrm{A23187}$ will efficiently and accurately equilibrate cytosolic free $\mathrm{Ca}^{2+}$ with extracellular free $\mathrm{Ca}^{2+}$. The validity of that assumption could not be assessed directly by measurements of the cytosolic level of free $\mathrm{Ca}^{2+}$ by Quin2 or Fura2, since the binding constants for $\mathrm{Ca}^{2+}$ of these fluorescent chelators do not allow accurate determination of free $\mathrm{Ca}^{2+}$ levels greater than 1 or $2 \mu \mathrm{M}[250,649$, 650]. Jy and Haynes [304] used a low concentration of A23187 $(0.5 \mu \mathrm{M})$ and a EGTA/Ca ${ }^{2+}$ buffer system to manipulate cytosolic free $\mathrm{Ca}^{2+}$. Using Quin2 they found that cytosolic free $\mathrm{Ca}^{2+}$ reflected extracellular free $\mathrm{Ca}^{2+}$ only in the range around the $\mathrm{kd}$ of Quin2. In our experiments, accurate equilibration of cytosolic and extracellular free $\mathrm{Ca}^{2+}$ is suggested by the abundant transport capacity of A23187 as documented by the fast rise of calpain activity observed after addition of A23187 to platelets. In addition, halfmaximal activity of calpain is observed at about $15 \mu \mathrm{M}$ free $\mathrm{Ca}^{2+}$, as estimated from the protein patterns. This value is in excel- lent agreement with values obtained with purified calpain-I.

Platelets are known to contain two isomers of calpain, caipain-I which is active at micromolar levels of free $\mathrm{Ca}^{2+}[212,333,552,655$, $656,679]$, and calpain-II which is active at millimolar levels of free $\mathrm{Ca}^{2+}[648,656,720]$. Halfmaximal activity of calpain-I is obtained at free $\mathrm{Ca}^{2+}$ levels ranging from 1 to $25 \mu \mathrm{M}$. Our experiments with intact platelets revealed $\mathrm{Ca}^{2+}$-dependent cytoskeletal degradation with halfmaximal activity at about $15 \mu \mathrm{M}$ free $\mathrm{Ca}^{2+}$ as estimated from the protein patterns, supporting the notion that Calpain- $\mathrm{l}$ is the major calcium-dependent protease in human platelets [656].

A peculiar finding, especially with regard to the $\mathrm{Ca}^{2+}$ titration of calpain activity in intact platelets is that degradation of cytoskeletal proteins stabilized within 5 minutes. It has been published that, upon stimulation, calpain is subject to autolysis of its heavy subunit [514], also in platelets [212], resulting in a reduction in molecular weight of the catalytic subunit. In addition it was observed that upon exposure to free $\mathrm{Ca}^{2+}$, platelet calpain becomes very labile [552]. Therefore we assume that the termination of calpain activity in intact platelets within 5 minutes is due to autolysis, rendering calpain inactive. Another explanation could be that activated calpain becomes susceptible to inhibition by its endogenous inhibitor, calpastatin [134], also present in platelets [655].

The $\mathrm{Ca}^{2+}$-dependence of prothrombinase activity would suggest that collagen plus thrombin raise cytosolic free $\mathrm{Ca}^{2+}$ to $10-15 \mu \mathrm{M}$. Activation of platelets by thrombin has been observed to result in cytosolic free $\mathrm{Ca}^{2+}$ levels ranging from 1 to $6, \mathrm{M}$ as reported by Quin2 $[275,525,535,554,680]$ and Fura2 [509], or ranging from 7 to $12 \mu \mathrm{M}$ as reported by aequorin $[554,680]$. Activation of platelets by collagen has been shown to elevate cytosolic free $\mathrm{Ca}^{2+}$ levels to about $2 \mu \mathrm{M}$ as monitored by Quin2 [680], or to levels ranging from 3 to $7 \mu \mathrm{M}$ as reported by aequorin $[448,680]$. Studies with electrically permeabilized platelets indicated that $\mathrm{Ca}^{2+}$ stimulated serotonin release is maximal at about $10 \mu \mathrm{M}$ free $\mathrm{Ca}^{2+}[273,327]$. Taking these summarized data into account, a level of 10 to $15 \mu \mathrm{M}$ cytosolic free $\mathrm{Ca}^{2+}$, resulting from stimulation of platelets by collagen plus thrombin, seems feasible considering the 
syniergistic action of both potenit platelet stimulators.

The close relationship between calpain activity and prothrombinase activity with regard to time course of development as well as $\mathrm{Ca}^{2+}$ requirement is consistent with a previous study by Comfurius et.al. [124]. They found the same order of potency for various platelet stimulators to evoke procoagulant activity and calpain-induced cytoskeletal breakdown. Extensive studies have demonstrated that prothrombinase activity reflects the presence of phosphatidylserine at the outer surface of the platelet plasma membrane $[48,50,543,733]$. The present findings may therefore be interpreted as a relationship between exposure of phosphatidylserine and cytoskeletal degradation by calpain.

In erythrocytes a similar relationship between the loss of cytoskeletal integrity and the loss of asymmetric transbilayer distribution of phosphatidylserine is amply documented [104, $168,217,349,423,572,703]$. The major cytoskeletal protein of erythrocytes, spectrin, is thought to interact with anionic phospholipids $[112,404,425]$, contributing to the maintenance of their asymmetric transbilayer distribution [256]. In addition, transbilayer reorientation of phosphatidylserine is thought to involve the formation of particular sites in the membrane, at which phosphatidylserine can take part in fast transbilayer movement, once released from binding to spectrin $[39,526]$. A recent development with regard to the factors involved in regulation of transbillayer orientation of anionic phospholipids in erythrocytes, is the discovery of an ATP-dependent inward transport of these lipids $[144,585,643,721]$.

In platelets, the regulation of transbilayer asymmetry of anionic phospholipids, in resting platelets as well as upon platelet activation, is poorly understood. As yet, interactions between the major platelet cytoskeletal proteins and anionic phospholipids have not been demonstrated directly. Bearer et.al. $[28,29]$ showed that submembrane cytoskelletal structures change their appearance with the same time course as the appearance of anionic lipid at the platelet outer surface, supporting the notion that in resting platelets interactions between structural proteins and anionic phospholipids play a role in the maintenance of transbilayer asymmetry of these lipids, analogous to erythrocytes. The experiments presented in this paper and in a previous one [124], show that degradation of cytoskeletal proteins by endogenous calpain could disturb these cytoskeleton-membrane interactions, contributing to a loss of membrane lipid asymmetry. This suggests a role for calpain in the regulation of the mechanisms by which phosphatidylserine becomes exposed at the outer platelet surface thus increasing the procoagulant activity of platelets.

\section{ACKNOWLEDGEMENTS}

Mrs. W.M.A. Linskens is gratefully acknowledged for her excellent technical assistance. 


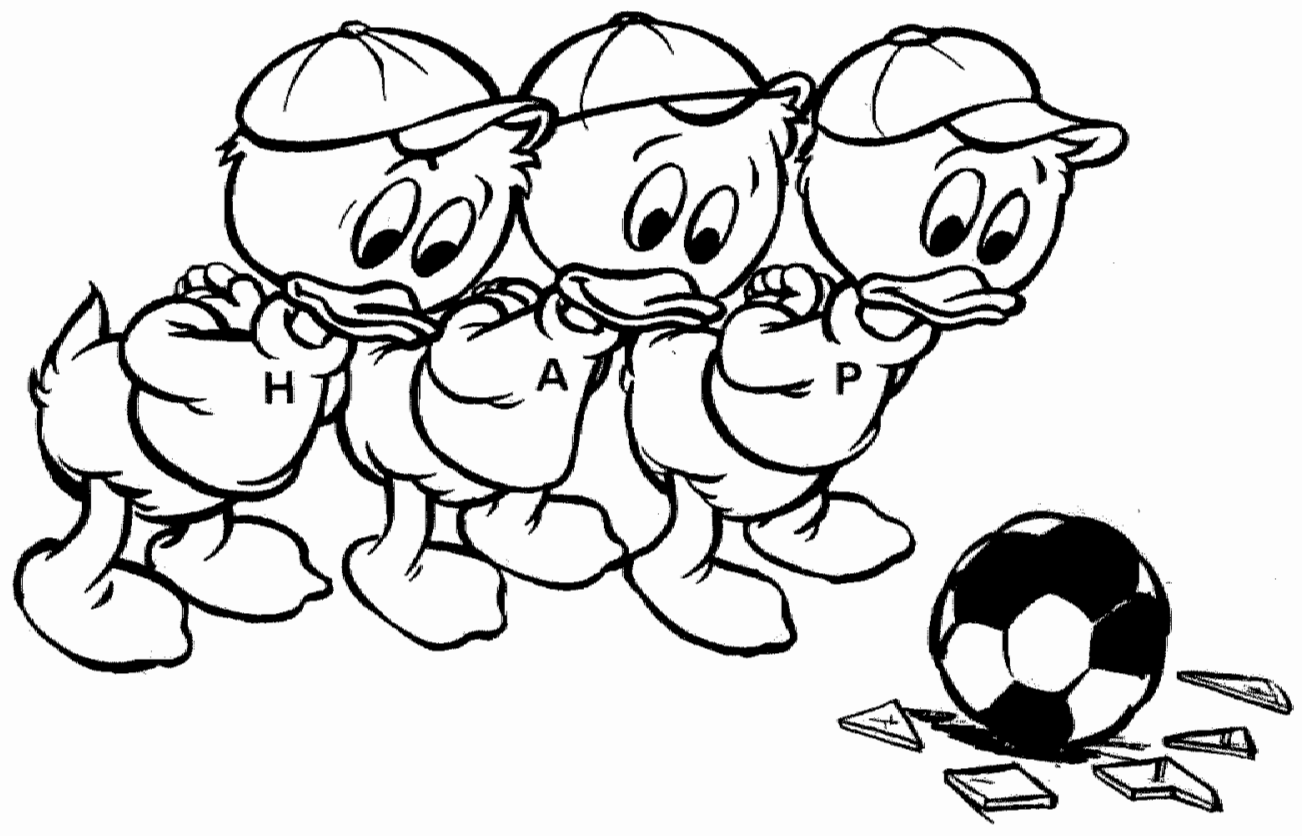



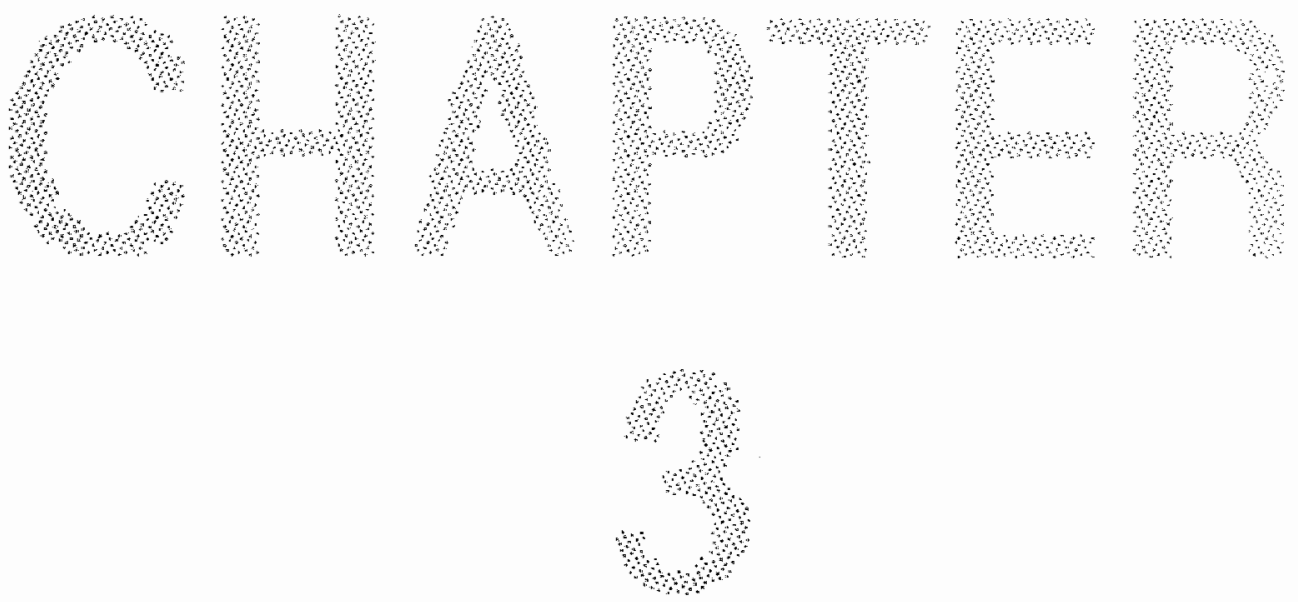


\title{
Relation between calpain and phospholipid transbilayer movement in fluoride-treated platelets.
}

\begin{abstract}
Treatment of platelets with fluoride $(10 \mathrm{mM})$ was found to result in a transient increase in $\mathrm{Ca}^{2+}$-permeability of the platelet plasma membrane. This phenomenon was used to provide supplementary evidence for the suggestions made earlier [Comfurius et.al.(1985), Biochim.Biophys.Acta, 903: 206; Verhallen et.al .(1987), Biochim.Biophys.Acta, 815: 143], that cytoskeletal disrupture by calpain is involved in the process leading to transbilayer movement of phosphatidylserine during expression of platelet procoagulant activity. This was achieved by relating both calpain activity and exposure of phosphatidylserine with platelet procoagulant activity. It was found that only upon addition of extracellular $\mathrm{Ca}^{2+}$ to fluoride-treated platelets, procoagulant activity, expressed as prothrombinase activity, and calpain activity, estimated from protein patterns after gel ellectrophoresis, were generated. Both $\mathrm{Ca}^{2+}$-inducible prothrombinase activity and calpain activity followed an identical time course during incubation with fluoride: after a time lag of about 10 min they sharply increased towards a peak level. Upon further incubation with fluoride, both activities decreased towards a final plateau, still above basal level. The presence of leupeptin during incubation with huoride was found to inhibit $\mathrm{Ca}^{2+}$-inducible calpain activity and prothrombinase reactivity in an identical way. $\mathrm{Ca}^{2+}$-inducible exposure of phosphatidylserine, as determined with extracellular phospholipase $A_{2}$, showed a similar pattern as $\mathrm{Ca}^{2+}$-inducible calpain activity and prothrombinase activity. From the strict parallelism between prothrombinase activity, calpain activity and exposure of phosphatidylserine, it is concluded that calpain plays an important role in the activation-dependent transbilayer movement of phosphatidylserine during expression of platelet procoagulant activity. It is suggested that degradation of the platelet membrane skeleton by calpain disturbs the structural organization of the lipid bilayer of the platelet plasma membrane leading to enhanced transbilayer mowement of phospholipids and appearance of phosphatidylserine at the platelet outer surface.
\end{abstract}




\section{INTRODUCTION}

Blood platelets have a multifaceted function in the hemostatic process. They initiate hemostatic plug formation upon vessel wall injury, and they promote fibrin formation by the coagulation cascade. In order to perform these functions platelets exhibit a wide variety of cellular responses. Primary hemostatic plug formation requires platelet adhesion, aggregation, and exocytosis. Activated platelets accelerate coagulation by increasing the rate of two sequential coagulation reactions: conversion of factor $X$ to $X_{a}$ by a complex of factors $I X_{a}$ and VIII $\mathrm{a}$, and the conversion of prothrombin to thrombin by a complex of factors $X_{a}$ and $V_{a}$ (prothrombinase complex). This property of stimulated platelets, referred to as procoagulant activity, is catalytic in nature [543a, 544], and can be interpreted as an increased number of binding sites for both the factor $\mathrm{X}$ activating complex and the prothrombinase complex [543a, 737].

In previous papers, we presented extensive evidence that anionic phospholipids present at the outer surface of activated platelets are the major determinant of the binding sites for the prothrombinase complex $[53,732,733]$. Using phosholipid vesicles of various compositions, it was suggested that phosphatidylserine is the physiologically relevant negatively charged phospholipid in both coagulation reactions [53, $162,529,543 a, 729]$. This notion was further substantiated by the effect of phospholipases on the procoagulant actiwity of resting, and stimulated platelets. These studies showed that only those phospholipases that can hydrolyse phosphatidylserine will decrease platelet procoagulant activity $[51,543 \mathrm{a}]$, and that stimulation of platelet procoagulant activity by a variety of agonists is related to the amount of phosphatidylserine exposed at the outer surface after platelet activation [50].

The plasma membrane of a resting platelet displays an asymmetric distribution of phospholipids [50, 98, 494, 569, 729]. Most extreme is the distribution of phosphatidylserine and sphingomyelin, which are almost exclusively located at the cytoplasmic and extracellular face of the platelet plasma membrane, respectively. To explain the increased exposure of phosphatidylserine after stimulation of platelet procoagulant activity, it is assumed that trans- bilayer movement of phospholipids (flipflop) is greatly enhanced during stimulation of platelet procoagulant activity $[50,730]$. This flipflop process is thought to produce randomization of phospholipids in the bilayer membrane, since in the outer leaflet exposure of phosphatidylserine is counterbalanced by a loss of sphingomyelin.

At present, the cellular mechanisms responsible for increased exposure of phosphatidylserine during expression of platelet procoagulant activity are poorly understood. In search of such mechanisms, we observed a striking correlation between the potency of various agonists to induce exposure of phosphatidylserine and to stimulate platelet calpain (endogenous calcium-dependent protease, EC.3.4.22.17) resulting in degradation of major cytoskeletal proteins (filamin, talin, and myosin) [124]. The relation between platelet procoagulant activity and calpain activity was further documented by comparing the time course of their development, their dose-response relationship, and their $\mathrm{Ca}^{2+}$ dependence [664].

During studies concerning the long term effect of metabolic ATP depletion on transbilayer phospholipid asymmetry in platelets, using sodium fluoride as a metabolic inhibitor, we observed that during incubation with fluoride for several hours, platelets temporarily exhibited an increase in platelet procoagulant activity. This reversible time course appeared to reflect a transient increase in $\mathrm{Ca}^{27}$-permeability of the platelet plasma membrane during incubation with fluoride. In this paper, the reversibility of fluoride-dependent $\mathrm{Ca}^{2+}$-inducible platelet procoagulant activity is used to provide supplementary evidence for the suggestions made earlier that calpain activity coincides with exposure of phosphatidylserine. This is achieved by relating both calpain activity and exposure of phosphatidylserine with platelet procoagulant activity expressed as prothrombinase activity. In addition, the effect of leupeptin (an oligopeptide inhibitor of calpain) on the relation between calpain- and prothrombinase-activity was explored.

\section{MATERIALS AND METHODS}

Sodium fluoride was from Merck (Darmstadt, FRG). Leupeptin, (acetyl-L-leucyl-L-leucyl-L-arginal) was 
from Sigma (St.Lowis, USA). Blood cargulation factors $\mathrm{Va}_{\mathrm{a}} \mathrm{Xa}$, prothrombin were isolated and purified from bovine blood according to established procedures described elsewhere [543a]. S2238 (H-D-phenylalanyl-L-pipecolylL-arginine-p-nitroanilide dihydrochloride), was from Kabi Diagnostica (Stockholm, Sweden). Phospholipase A2 (phosphatide-2-acylhydrolase: EC 3.1.1.4) was purified according to $Z$ waal et,all. [736] from bee venom (Apis mellfica) obtained from Koch Light Laboratories (Colnbrook, UK). FURA-2 AM was from Molecular Probes Inc (Eugene OR, USA).

\section{Platelet isolation}

Washed human platelets were isolated by differential centrifugation as described prewiously [51] from fresh blood, drawn from healthy vollunteers receiving no medication. Blood was anticoagulated with acid-citrate-dextrose. Washed platelets were resuspended in an isotonic buffer at pH 7.4, containing $10 \mathrm{mM}$ Hepes (4-(2hydroxyethyl)-1,-piperazineethanesulfonic acid), $136 \mathrm{mM} \mathrm{NaCl}$, $27 \mathrm{mM} \mathrm{KCl}, 2 \mathrm{mM} \mathrm{MgCl}, 5 \mathrm{mM}$ glucose and $0.05 \%$ fatty acid-firee human serum albumin (Hepes buffer). Platelet concentration was inferred from their absorption at 405 nm using a calibration curve determined with a Coulter Counter. Platelet lysis was determined by assaying lactate debydrogenase (EC 1.1.1.27) in a $12000 x g$ supermatant according to Wroblewski and La Due [710].

\section{Measurement of platelet procoagulant activity}

Platelet procoagulant actîvity was detennined as described earlier [ 5433,664$]$ by assaying prothrombinase activity under conditions at which the procoagulant surface is rate limiting. The assay was carried out at $37^{\circ} \mathrm{C}$ in Hepes buffer under gentle stirring ( $250 \mathrm{rev} / \mathrm{min}$ ). To monitor the effect of fuoride on platelet procoagulant activity, platelets $\left(210^{8} \mathrm{ml}^{-1}\right)$ were incubated with $10 \mathrm{mM}$ fluoride at $37^{\circ} \mathrm{C}$, without stirring to prevent aggregation. Immediately before the addition of fluoride, 1 mM EGTA was added to sequester trace amounts of $\mathrm{Ca}^{2+}$. At different time intervals a $15 \mu$ alliquot of the luoride incubation was dilluted twentyfodd into Hepes buffer containing $3 \mathrm{mM}$ $\mathrm{CaCl} 2$ No change in $\mathrm{pH}$ was apparent upon this dilution. After 1 min factor $\mathrm{Va}(6 \mathrm{nM}$ final conc) and factor $\mathrm{Xa} \mathrm{(3}$ mM final conc) were added, and 1 min later the prothrombinase reaction was started by addition of prothrombin (final conc. $4 \mu \mathrm{M}$, final assay wolume $0.5 \mathrm{mil}$ ). The amount of thrombin formed $1 \mathrm{~min}$ after the addition of prothrombin enzymatically determined using the thrombin-specific chromogenic substrate $\$ 2238$.

Occasionally, the time course of thrombin formation after $\mathrm{Ca}^{2+}$-addition was monitored by assaying thrombin activity in an aliciout taken every 10 sec after addition of Puoride treated platelets to the complete prothrombinase mixture.

\section{Determination of $\mathrm{Ca}^{2+}$ influx using Fura-2}

Platelets were loaded with Fura-2 according to Rao et.al. [525]. Platelets pelleted from platelet rich plasma were resuspended in Hepes buffer to approximately $310^{8}$ $\mathrm{ml}^{-1}$ and incubatied with $1 \mathrm{HM}$ Fura-2 AM for 30 min at $37^{\circ} \mathrm{C}$. After loading platelets were ashed as described under platelet isolation. The effect of fuoride on $\mathrm{Ca}^{2+}$ influx into Fura-2 loaded platelets was monitoned at different time intervals after tenfold dilution in Hepes buffer of an aligout of platelets incubated with Auorido $\left(210^{8}\right.$ pl.mrl $10 \mathrm{mM}$ fluoride, $1 \mathrm{mM}$ EGTA, $37 \mathrm{C}) .3 \mathrm{mMCa} \mathrm{Ca}^{2}$ was added under continuous bingh speed reconding ( $5 \mathrm{sec}$ per inch) of Fura-2 fluorescence. The initial rate of change in fuorescence signal after $\mathrm{Ca}^{2}$ addition was taken to reflect quantitatively the rate of $\mathrm{Ca}^{2+}$ innux. Fluorescence intensities (F) were reoorded at $37^{\circ} \mathrm{C}$ under continuous stirring ( $400 \mathrm{rev} / \mathrm{min}$ ) with an SLM Aminco SPF-500C fluorimeter equipped with a lightitight injection port and a stirring device (Hellma). Excitation and emission wavelengths were set at 335 and $500 \mathrm{~nm}$ with 5 and $20 \mathrm{~nm}$ slitts, respectiwely.

\section{Determination of calpain activity by gel electro- phoresis}

Calpain activity in platelets was estumated from protein paiterns after gel electrophorestis, by monitoring degradation of substrates and formation of fragments, as described in detail [664]. Samples for gel ellectrophoresis were taken simultameously with samples for the prothrombinase assay, from the same incubation of platelets with fluoride, at various time intervals. They were diluted tenfold in albumin-free Hepes buffer, containing $3 \mathrm{mM}$ $\mathrm{Ca}^{2+}$, fier 3 min calpain activity was arrested by addition of Hepes buffer containing protease inhibitors and excess EGTA. Disc electrophoresis was performed according to Laemmil [356] on 6-12\% gradient polyacrylamide slab gels. Gels were stained with Coomassie brilliant bluc. Quantitative analysis of protein patterns was performed by densitometric scanning of stained gels (LKB 2202 U1troscan laser densitometer, equipped with an integrator). Calpain activity was expressed as the amownt of fragments (at 190,135 and $95 \mathrm{kDa}$ ) relatike to total protein. To eliminate differences due to differences in amount of total protein, the amount of actin was taken as an internal standard.

\section{Determination of exporure of phosphatidylserine}

The cxposure of phosphatidylserine was inferied from its accessibility towards excess extracellular phospholipase $\mathrm{A}_{2}$ from bee venom. Samples for phospholipase treatiment were taken simultaneously with samples for the prothrombinase assay from the same incubation of platelets with thoride $\left(510^{8} \mathrm{plm}^{-11}, 10 \mathrm{mM}\right.$ fluoride, $1 \mathrm{mM}$ ECTA). After tenfold dilution into Hepes buffer containing 10 $\mathrm{mM} \mathrm{Ca}^{2+}$, phospholipase $\mathrm{A}_{2}$ was addexil to a finall concentration of 2 IU.m. ${ }^{-1}$. Every 4 min a subsimple $\left(10^{9}\right.$ platelets) from the phospholipase Az incubation was thaken for examination of phospholipid degradation and determina tion of platelet lysis. Phospholipase activity an in hibited by addition of 20 mM EGTA to the subsample, prior to the extraction of lipids accorting to Bligh and Dyer [61]. Phospholipid anallytis was carried out by twodimensional thin layer chromatography followed by phos phorus determination as described by Verkleij et.al. [666]. 


\section{RESULTS}

\section{Fuoride-dependent $\mathrm{Ca}^{2+}$-induced platelet procoagulant activity}

Changes in $\mathrm{Ca}^{2+}$-permeability of the platelet plasma membrane during incubation with fluoride were investigated employing Fura2loaded platelets. The initial change in Fura2 signal after addition of extracelluilar $\mathrm{Ca}^{2+}$ was taken to represent $\mathrm{Ca}^{2+}$-influx quantitatively. It was found that the entry of extracellular $\mathrm{Ca}^{2+}$ into the platelet cytosol was transiently facilitated during the course of a two-hour incubation of platelets with fluoride (Fig. 1.A).

The effect of fluoride $(10 \mathrm{mM})$ on platelet procoagulant activity was determined by assaying prothrombinase activity in subsamples taken from an incubation of platelets with fluoride. It was found (Fig. 1B) that during incubation with fluoride prothrombinase activity sharply increased, after a time lag of 10 to 15 min, to a maximum level of approximately 700 niM thrombin per min. After reaching this maximum, prothrombinase activity gradually decreased upon progressive incubation with fluoride, to level off at about one-third of its peak level. Except for the time lag, the transient character of $\mathrm{Ca}^{2+}$-inducible prothrombinase activity coincides with the transient increase in $\mathrm{Ca}^{2+}$-permeability during fluoride treatment. Under the same conditions of fluoride treatment, platelet aggregation and ${ }^{3} \mathrm{H}-$ serotonin release started after a delay of 5 minutes and levelled off at about $10 \%$ and $65 \%$, respectively, within 15 to 20 min after addition of fluoride.

It is emphasized that fluoride treatment is carried out in the absence of extracellular $\mathrm{Ca}^{2+}$, whille prothrombinase activity is obligatory measured in the presence of extracellular $\mathrm{Ca}^{2+}$. Considering the importance of extracellular $\mathrm{Ca}^{2+}$ for the expression of platelet procoagulant activity $[124,664]$ and the effect of fuoride treatment on $\mathrm{Ca}^{2+}$ permeability of the platelet plasma membrane, the question arose whether the observed procoagulant activities were induced during incubation of platelets with fluoride in the absence of $\mathrm{Ca}^{2+}$, or whether they were induced by influx of extracellular $\mathrm{Ca}^{27}$ present during the prothrombin- ase assay. Therefore, the time course of thrombin formation during the prothrombinase assay was determined and recalculated as generation of prothrombinase activity [664]. It appeared that the procoagulant activity of fluoridetreated platelets was actually generated by extracellular $\mathrm{Ca}^{2+}$ during the prothrombinase assay, reaching its maximum level within $2 \mathrm{~min}$ (inset Fig. 1B), i.e. before addition of prothrombin.

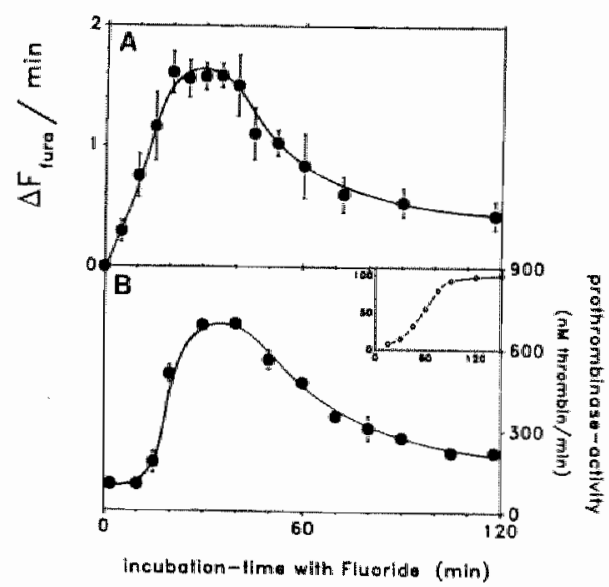

Fig. 1. Time course of $\mathrm{Ca}^{2+}-$ influx (A) and prothrombinase activity (B) during incubation of plattelets with fluoride. Both parameters were evaluated after addition of $3 \mathrm{mM} \mathrm{Ca}{ }^{2+}$ to platelets incubated with $10 \mathrm{mM}$ fluoride, as described under Methads. The curve for $\mathrm{Ca}^{2+}-$ influx $(A)$, represented by the initial change in Fura -2 fluorescence ( $\Delta F_{\text {furd }} /$ min) after $\mathrm{Ca}^{2+}$ addition, is the mean ( \pm SEM) of 5 experiments. The curve for prothrombinase activity $(B)$ was constructed from 9 experiments in duplo (mean \pm SEM). In general, the rewersible time course of both effects of fluoride on platelets was highly reproducible, though peaktime and peak-width differed between different platelet preparations. Platelet lysis was negligible during the two hours incubation with fluoride. Inser $(B)$ : Generation of prothrombinase activity immediately after addition of $\mathrm{Ca}^{2+}$ to plateiets incubated with fluoride for $30 \mathrm{~min}$. Ordinate: prothrombinase activity expressed as percentage of maximal activity. Abscissa: time (sec) after $\mathrm{Ca}^{2+}$ addition. It appeared that throughout the whole incubation of platelets with fluoride, $\mathrm{Ca}^{2+}$-induced generation of prothrombinase activity followed the same time course (not shown). 
Fuoride-dependent $\mathrm{Ca}^{2+}$-induced exposure of phosphatidylserine.

Phospholipase $\mathrm{A}_{2}$ from bee venom was used to determine the amount of phosphatidylserine exposed at the outer surface of fluoride-treated platelets after $\mathrm{Ca}^{2+}$-exposure. This approach is based on the observation that under non-lytic conditions only phospholipids present in the outer leaflet of the plasma membrane can be hydrolysed by exogenous phospholipase $A_{2}[50,98,539,736]$. Fluoridle-dependent $\mathrm{Ca}^{2+}$-induced exposure of phosphatidylserine was related with prothrombinase activity at three characteristic time points during fluoride treatment: at the start, at the peak level and at the lower final level of prothrombinase activity. In order to determine the saturating level of hydrolysis by phospholipase $A_{2}$ the time course of hydrolysis of both phosphatidylserine and total phospholipids was determined. In this way changes in hydrolysis of phosphatidylserine can be compared with changes in hydrolysis of total phospholipids. It was found that at peak levels of prothrombinase activity, accessibility of phosphatidylserine towards phospholipase $\mathrm{A}_{2}$ was

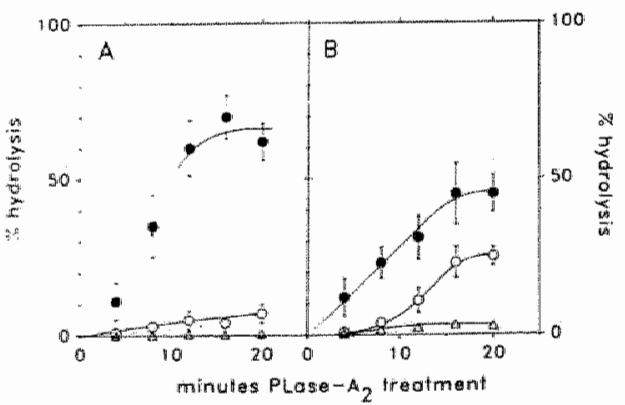

Fig. 2. Hydrolysis of phosphatidylserine (A) and total phospholipids (B) in fluoride-treated platelets, after exposure to $\mathrm{Ca}^{2+}$. Degradation of phospholipids in the outer membrane of fluoride-ireated platelets by phospholipase $A_{2}$ from thee wenom was examined $0 \mathrm{~min}(\Delta), 30 \mathrm{~min}(\odot)$ and $120 \mathrm{~min}(O)$ after the addition of fluoride, as outlined in the Methods. The data shown are the mean ( \pm SEM) of 3 experiments. During treatment with phospholipase A2, platelet lysis gradually increased to a maximum of $10 \%$. Data shown were corrected for phospholipid hydrolysis in lysed cells [124]. The presence of residual fluoride during treatment with phospholipase $A_{2}$ was found not to affect phospholipase $A_{2}$ activity. dramatically increased to approximately $65 \%$, compared to less than $1 \%$ and $10 \%$ at the start and at the end of the incubation with fluoride, respectively (Fig. 2A). In contrast, hydrolysis of total phospholipids was only two-fold increased at peak level prothrombinase activity in comparison with the hydrolysis at the end of fluoride treatment (Fig. 2B). At the start of fluoride treatment hardly any hydrolysis of phospholipids was observed, in agreement with earlier observations $[49,98]$.

\section{Fluoride-dependent $\mathrm{Ca}^{2+}$-induced calpain activity}

The relationship between calpain activity and prothrombinase activity was determined by applying a simnilar $\mathrm{Ca}^{2+}$-exposure after fluoride treatment. From the protein patterns (Fig. 3C) and their quantitative analysis (Fig. 3B), it is obvious that calpain activity followed the same reversible time course as prothrombinase activity: a fast rise after a time lag, followed by a gradual decline. In addition, when extracellular $\mathrm{Ca}^{2+}$ was omitted, no calpain activity could be demonstrated in fluoride-treated platelets (Fig. 3 C). Generation of prothrombinase activity in fluoride-treated platelets was similarly dependent on extracellular $\mathrm{Ca}^{2+}$ (Fig. 1B).

In search of a direct demonstration of the involvement of calpain in $\mathrm{Ca}^{2+}$-activated platelet procoagulant activity, we investigated the effect of leupeptin (inhibitor of calpain) on the relation between fluoride-dependent $\mathrm{Ca}^{2+}$-induced prothrombinase activity and calpain activity. It has been suggested that leupeptin crosses the platelet plasma membrane $[21,548]$. The presence of leupeptin $(0.5 \mathrm{mM})$ during incubation of platelets with fluoride resulted in a significantly decreased peak level of calpain activity, and the final low level was reached faster (Fig. $3 B_{4}, C$ ). An identical effect of leupeptin was observed on prothrombinase activity of platelets sampled from the same incubation with fluoride (Fig. 3A): Considering that leupeptin can inhibit thrombin [547a], the effect of leupeptin on the prothrombinase assay was investigated. At the concentration used (15 $\mu \mathrm{M}$ in the prothrombinase assay), leupeptin had no effect on the enzymatic activity of the prothrombinase complex in the presence of vesicles composed 
of 20 mole $\%$ phosphatidylserine and 80 mole\% phosplhatidylcholine (data not shown).

The parallelism between $\mathrm{Ca}^{2+}$-inducible prothrombinase activity and calpain activity was consistently abserved $(n=8)$. The effect of leupeptin on both prothrombinase activity and calpain activity was highly variable between differentl platelet preparations, and frequently difficult to demonstrate. However, on those oecasions that leupeptin did exhibit a clear effect $(\mathrm{n}=4)$, the time course of both $\mathrm{Ca}^{2+}$-induced prothrombinase activity and calpain activity during incubation of platelets with fluoride was identically affected.

\section{DISCUSSION}

In this paper we used fluoride-treated platelets to extend previous observations suggesting a role for calpain in the stimulus-response coupling of platelet procoagulant activity $[124$, 664]. During incubation with fluoride the

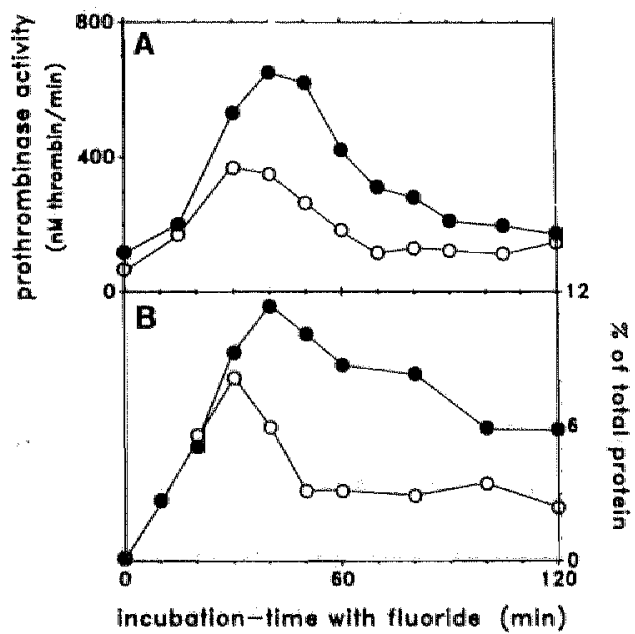

$\mathrm{Ca}^{2+}$-permeability of the platelet plasma membrane was increased in a reversible manner, causing platelet procoagulant activity, calpain activity, and exposure of phosphatidylserine to be enhanced with a similar time course.

At present the action of fluoride is not fully understood. Uptake of fluoride by platelets at physiological $\mathrm{pH}$ is maximal within 10 minutes [436], indicating that the effect of fluoride on $\mathrm{Ca}^{2+}$-permeability of the platelet plasma membrane during a two hour incubation should result from cellular processes secondary to fluoride. Fluoride is known to affect platelets in at least two different ways: 1) glycolysis is instantaneously and efficiently inhibited, leading to a fast decrease in metabolic ATP $[435,436$, 437], and 2) it activates various $G$-proteins, resulting in platelet activation $[76,317,504]$. Considering the irreversibility of fluoride-induced ATP depletion, the activation of G-proteins seems to be the best candidate to explain the reversible effect of fluoride on $\mathrm{Ca}^{2+}$-permeability of the platelet plasma membrane.

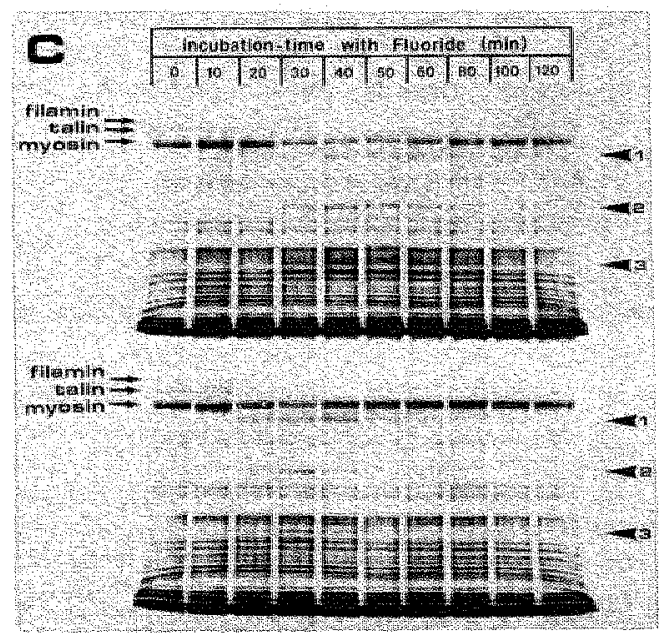

Fig. 3. Relation between $\mathrm{Ca}^{2+}$-induced prothrombinase activity (A) and calpain activity (B,C) of fuoride-treated platelets, and the effect of leupeptin on this relation. Platelets were incubated with 10 $\mathrm{mM}$ fluoride in the absence (C) or presence (O) of $0.5 \mathrm{mM}$ leupeptin. (B): Degradation of cytoskeletal proteins by calpain after addition of $\mathrm{Ca}^{2+}$ to fluoride-treated platelets, as judged by the formation of their fragments (sum of protein bands indicated at the right side of (C)). Data were quantitated from the gels shown in (C), after densitometric scanning as outlined in the methods. Changes in the amount of fragnents were counterbalanced by changes in the amount of substrates (not shown). (C) Upper and lower panel: time course of $\mathrm{Ca}^{2+}$-inducible calpain activity during incubation with $10 \mathrm{mM}$ fluonide, in the absence and presence of $0.5 \mathrm{mM}$ leupeptin, , respectively. Calpain activity is indicated by the disappearance of its substrates (indicated at the left), and the appearance of their fragments (indicated at the right). Samples fior gell electrophoresis were taken from the same incubation as that of which the $\mathrm{Ca}^{2+}$-induced prothrombinase activity was defermined. For experimental details is referred to the Methods. The results shown in this figure are taken from a representative experiment. 
Fluoride has been reported to increase platelet cytosolic $\mathrm{Ca}^{2+}$ levels, partly dependent of extracellular $\mathrm{Ca}^{2+}[76,324,504]$. Also in other cells fluoride has been shown to promote $\mathrm{Ca}^{2+}$-influx [183, 355, 619]. In addition, $\mathrm{G}-$ proteins are suggested to be involved in the regulation of $\mathrm{Ca}^{2+}$-channels $[244,282,311$, 355], and, in general, ion-channels form part of the effector targets of $\mathrm{G}$-proteins in cellular signal transduction [294]. Therefore, we assume that the transient facilitation of $\mathrm{Ca}^{2+}$-influx by fluoride is caused by the action of fluoride on $\mathrm{G}$-protein dependent $\mathrm{Ca}^{2+}$-channels in the platelet plasma membrane.

The involvement of G-proteins could explain the reversible nature of fluoride-dependent $\mathrm{Ca}^{2+}$-permeability of the platelet plasma membrane. Activation of G-proteins by fluoride has been shown to result from the action of aluminiumtetrafluoride (AlF4) [614], suggested to fill the space of the third phosphate group in the guanine-nucleotide binding pocket containing already GDP in the inactive state [57]. Extensive depletion of phosphorylated nucleotides during prolonged incubation with fluoride could result in depletion of GDP. Resynthesis of ATP from IMP at the cost of GTP has been demonstrated in fluoridetreated platelets [438]. Thus, it is possible that the decline in fluoride-dependent $\mathrm{Ca}^{2+}$-permeability of the platelet plasma membrane, after reaching peak level, is due to depletion of GDP resulting in inactivation of fluoridestimulated G-proteins and closure of $\mathrm{Ca}^{2+}$ channels.

$\mathrm{Ca}^{2+}$-permeability increases right from the start of fluoride treatment. However, calpain activity and prothrombinase activity were increased only after a time lag of 10 to $15 \mathrm{~min}$. Platelet aggregation and release during fluoride treatment were found to level off within 15 to $20 \mathrm{~min}$, in agreement with earlier reports [317, 436, 504]. This suggests that prothrombinase activity and calpain activity are dependent on higher cytosolic free $\mathrm{Ca}^{2+}$ levels than required for aggregation and release, confirming previous findings [664]. In addition, the discrepancy between the development of platelet aggregation and release, and the development of prothrombinase activity and calpain activity upon fluoride treatment, indicates that the cellular mechanisms generally shown to be involved in platelet stimulus-response coupling (phospholipase $C_{3}$ phospholipase $\mathrm{A}_{2}$ and pro- tein kinase C) are not directly responsible for the generation of increased phospholipid transbilayer movement during the expression of platelet procoagulant activity: This, is suggested by the activation of phospholipid hydrolysis [76, $317,504]$ and protein phosphorylation [317, 438 ] by fluoride during the first 10 to 20 min of incubation of platelets with fluoride, where $\mathrm{Ca}^{2+}$-inducible calpain activity and prothrombinase activity are not increased. These conclusions are in accordance with previous observations that thrombin, being a strong platelet agonist, is hardly able to induce procoagulant activity and calpain activity [124].

Peak level of fluoride-dependent $\mathrm{Ca}^{2+}$ induced prothrombinase activity is accompanied by increased accessibility of phosphatidylserine towards phospholipase $\mathrm{A}_{2}$ in comparison with unstimulated platelets and in comparison with platelets treated with fluoride for two hours. This increased accessibility of phosphatidylserine cannot be explained only by increased hydrolysis of total phospholipids. Thus, it is concluded that the ratio phosphatidylserine/total phospholipids in the outer monolayer of the platelet plasma membrane follows a similar reversible time course as the fluoridedependent $\mathrm{Ca}^{2+}$-induced prothrombinase activity. It is unlikely that exposure of phosphatidylserine results from fusion of granular membranes with the plasma membrane during the release reaction, since the release preceeds the development of prothrombinase activity in fluoride-treated platelets. Therefore phosphatidylserine must have crossed the lipid bilayer of the platelet plasma membrane considering its location in unstimulated platelets $[50,98$, $49.4,569]$.

The observation that phospholipase $A_{2}$ alone is able to hydrolyse phospholipids in the platelet plasma membrane only under those conditions where an increased prothrombinase activity is seen, confirms earlier findings [50]. The ability of phospholipase $\mathrm{A}_{2}$ to hydrolyse membrane lipids is greatly dependent on the molecular packing of the lipids, expressed as surface pressure, and may be enhanced by the presence of anionic phospholipids [157]. Increased ability of phospholipase $A_{2}$ to hydrolyse membrane lipids has been shown to correlate with increased merocyanine- 540 uptake of the membrane $[95,103,526]$, indicating a decreased order of membrane lipids. Therefore, we suggest that the increased hydrolysis of total 
phospholipids at peak level of fluoride-dependent $\mathrm{Ca}_{2}{ }^{+}$-induced prothrombinase activity reflects both changes in membrane lipid organization, as they are thought to occur during enhanced transbilayer movement of phospholipids [154], and the presence of anionic phospholipids in the outer monolayer of the platelet plasma membrane.

The observation that fluoride-dependent $\mathrm{Ca}^{2+}$-induced callpain activity follows the same reversible time course as prothrombinase activity, clearly extends previous observations suggesting a relation between calpain activity and platelet procoagulant activity $[124,664]$. In particular, the identical effect of leupeptin on both fluoride-dependent calpain activity and prothrombinase activity provides a strong argument for a role of calpain in the transbilayer movement of phosphatidylserine during expression of platelet procoagulant activity, considering that calpain is the major $\mathrm{Ca}^{2+}$-dependent protease in platelets [648].

The rellation between calpain activity and exposure of phosphatidylserine does not unequivocally imply that cytoskeletal proteins are the important substrates of calpain involved in regulation of transbilayer movement of phosphatidylserine. However, it has been shown (1) that the substrate specificity of calpain is mainly confined to cytoskeletal proteins, in general $[431,514,682]$ as well as in platelets $[216,499$, $608,656,690]$, and (2) that the organization of cytoskeletal proteins plays an important role in the regulation of transbilayer asymmetry of anionic phospholipids in the erythrocyte $[217,256$, 423]. Therefore, we suggest that it is the degradation of cytoskeletal proteins by calpain which is causally related to transbilayer movement of phosphatidylserine during expression of platelet procoagulant activity.

The involvement of calpain mediated cytoskeletal degradation in the regulation of transbilayer movement of phosphatidylserine during expression of platelet procoagulant activity can be understood by applying the current model of the regulation of phospholipid transbilayer asymmetry in the erythrocyte. In erythrocytes the asymmetric distribution of phospholipids over the plasma membrane is thought to be maintained 1) by interaction of the lipid bilayer with cytoskeletal proteins $[168,256]$ located underneath the plasma membrane (membrane skeleton) [72,114,249,402], and 2) by the ATP-dependent action of an aminophos- pholipid-specific translocase $[144,585,643$, 721]. The loss of phospholipid transbilayer asymmetry in the erythrocyte involves 1) a reorganization of the membrane skeleton, disabling the interaction with plasma membrane phospholipids $[104,217,349,423,703]$, and 2) an increased transbilayer movement of phospholipids via disturbances of the lipid bilayer structure $[39,218,219]$.

It is becoming increasingly evident that platelets, besides a phospholipid transbullayer asymmetry similar to erythrocytes $[50,98,494$, $569,729]$, also contain a membrane skeleton $[147,207,215,331,471,472,482,546]$, functionally similar to that of the erythrocyte [204]. Therefore, the role of calpain-mediated cytoskeletal degradation in the transbilayer movement of phosphatidylserine during expression of platelet procoagulant activity (i.e. the loss of phospholipid transbilayer asymmetry), is thought to be disabling the interaction between membrane skeleton and membrane lipids, thereby enabling the phospholipids to participate in transbilayer movement. However, it may well be that the loss of interaction between membrane skeleton and plasma membrane, by itself, produces local disturbances in the lipid bilayer (flipsites) via which transbilayer movement of phospholipids can proceed at enhanced rates. This suggestion has been put forward earlier $[39,219,423]$ and is documented by observations that changes in the organization of the membrane skeleton are accompanied by changes in the structural organization of the lipid bilayer of the plasma membrane, in erythrocytes $[15,95,103,194,526,703]$ as well as in platelets [28].

The notion that the action of calpain towards the membrane skeleton can produce disturbances in the organization of the lipid bilayer would explain the observed increase in hydrolysis of membrane phospholipids by phospholipase $A_{2}$ when platelet procoagulant activity is increased [50], considering the dependence of phospholipase $A_{2}$ activity on the lipid packing [157]. The dual role of calpain-mediated degradation of the membrane skeleton in the loss of phospholipid transbilayer asymmetry, both in enabling aminophospholipids to participate in transbillayer movement and in inducing local disturbances in lipid bilayer organization, is further evidenced by the observation that hydrolysis of the erythrocyte membrane skelleton by calpain leads to increased transbi- 
layer movement of phosphatidylethanolamine and phosphatidylserine, accompanied by disturbances of the lipid bilayer [95].

Fast outward transbilayer movement of phospholipids may not be dependent on disturbances of the lipid bilayer (fllipsites), since it is recently proposed that the ATP-dependent translocase functions bidirectionally (flippase) [702]. However, to explain fast outward transbilayer movement of phosphatidylserine by the action of a flippase, finished within $2 \mathrm{~min}$ after addition of $\mathrm{Ca}^{2+}$ to fluoride-treated platelets, one must assume that the ATP requirement of the flippase is fullfilled already at very low ATP levels, considering the prolonged metabolic inhibition by fluoride. If ATP is not necessarily hydrolysed during flippase action, it may be that ADP/AIF4" is able to sustain flippase action, analogous to the activation of $\mathrm{G}$-proteins by GDP/AlF 4 :

\section{ACKNOWLEDGEMENTS}

Mrs. W.M.A Linskens is gratefully acknowledged for her excellent technical assistance. 

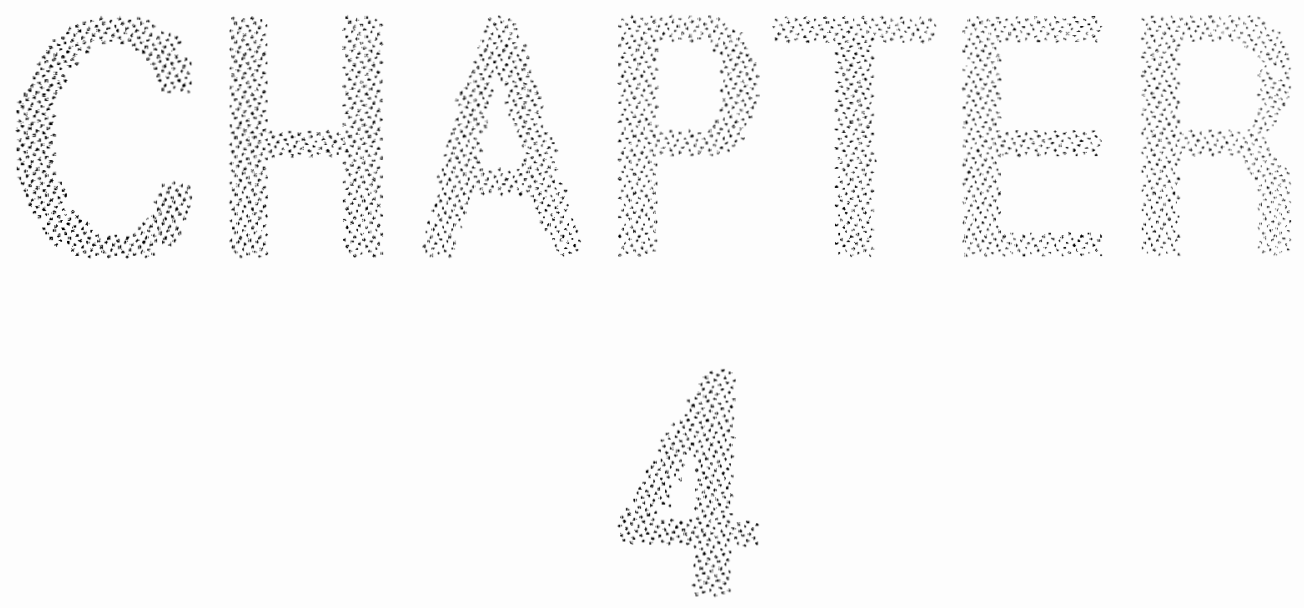


\title{
Phospholipid transbilayer asymmetry in artificially induced platelet plasma membrane vesicles.
}

\begin{abstract}
Incubation of human platelets with unilamellar vesicles composed of dilauroylphosphatidylcholine (DLPC) induced shedding of small vesicular structures from the platelet plasma membrane. No significant cell lysis is observed during the process of shedding. Isollated spicules contain the major membrane glycoproteins, $\mathrm{I}_{\mathrm{b}} \mathrm{II}_{\mathrm{b}}$, and $\mathrm{III}_{\mathrm{a}}$, which are used to define the sidedness of the spicule membrane. These glycoproteins are completely susceptible to chymotrypsin treatment, whereas cytoskeletal proteins are inaccessible towards this enzyme. This demonstrates that the spicule membranes have a right-side-out orientation in as far as membrane proteins are concerned. Isolated spicules were 30 fold more active than platelets in stimulating prothrombin conversion to thrombin by the prothrombinase complex (factors $X_{a}, V_{a}$, and $\mathrm{Ca}^{24}$ ). The increased prothrombinase activity reflects an increased amount of phosphatidylserine in the outer leanet of the spicule membrane. Protein analysis of platelet spicules and native platelets reveals a number of differences, the most conspicuous of which is the virtual absence of myosin in the spicule preparations. It is proposed that a lack of myosin produces a different cytoskeletal organization in the spicules. This enables phosphatidylserine to become exposed at the outer surface of the spicule membrane.
\end{abstract}




\section{INTRODUCTION}

As reviewed by Op den Kamp [476], asymmetric distribution of phospholipids is a common feature of most, if not all, biological membranes. In the plasma membrane of platelets the negatively charged phospholipids phosphatidylserine (PS) and phosphatidylinositol (PI) are mainly confined to the cytoplasmic leaflet, whereas sphingomyelin (SphM) is predominantly located in the outer half of the membrane. In contrast to the rather extreme distribution of these phospholipids, phosphatidylcholine (PC) and phosphatidylethanolamine (PE) are present on either side of the membrane, though not to the same extent [98, $494,568,729]$.

Upon activation of platelets, in particular by collagen plus thrombin or by the non-physiological $\mathrm{Ca}^{2+}$ ionophore $\mathrm{A} 23187$, the non-random distribution of phospholipids is partially lost, which results in an increased exposure of PS at the outer surface of the platellet $\left[49_{n} 50\right]$. The increased exposure of PS is reflected by an increased capability of platelets to enhance two sequential reactions of the coagulation cascade, i.e. the activation of factor $X$ by a compllex of factors $\mathbb{L X}_{\mathrm{a}}$ and $\mathrm{VII}_{\mathrm{a}}$ and the activation of prothrombin by a complex of factors $X_{a}$ and $V_{a}$ $[49,50,543 a]$. The rate of both coagulation reactions is critically dependent on the presence of a negatively charged phospholipid surface. A close correlation was found between platelet procoagulant activity and exposure of PS at the platelet outer surface [50].

Exposure of PS in the outer leaflet of the platelet plasma membrane presumably results from an increased transbilayer movement of phospholipids (flipflop) [49, 50]. Recent data demonstrated that an increased exposure of PS strictly coincides with an increased breakdown of several cytoskeletal proteins by the action of calpain, an endogenous $\mathrm{Ca}^{2+}$-dependent protease in platelets [124]. From this it was suggested that, in analogy to the erythrocyte membrane $[217,218,220,256,259,423,703]$, interactions between membrane phospholipids and underlying cytoskeletal proteins play an important role in regulation of membrane phospholipid asymmetry.

Recently, Kobayashi and co-workers [330] found that spicules, derived from rabbit platelets upon incubation with dilauroylphospha- tidylcholine (DLPC) vesicles, were essentially devoid of a $200 \mathrm{kDa}$ protein, representing the heavy chain of myosin, one of the major cytoskeletal proteins in platelets. The aim of the present study was to investigate whether similar spicules could also be derived from human platelets and to see if and to what extent an alteration in composition of cytoskeletal proteins is accompanied by a change in phospholipid distribution in the membranes of these spicules. The ability of the spicules to enhance the activation of prothrombin by a complex of factors $\mathrm{X}_{\mathrm{a}}$ and $\mathrm{V}_{\mathrm{a}}$ (prothrombinase activity) was used to monitor the exposure of PS in the outer leaflet of the spicule membrane.

\section{MATERIALS AND METHODS}

Dilauroylhosphatidylcholine, a-chymotrypsin, fatty acid-free human serum albumin, peroxidasemconjugated concanawalin A and peroxidase-conjugatted wheat germ agglutinin were purchased from Sigma Chemical Co. (St. Louis, USA). The prostacyclin analogue $Z K$ 36374 was a kind gift of schering $A G$ (Berlin, BRD). $H$ D-phenylalanyl-Lmpipecolyl-L-arginine-p-nitroanilide dihydrochloride ( $\$ 2238$ ), a chromogenic substrate specific for thrombin was from AB Kabi Diagnostica (Stockbolm, Sweden). All other reagents were of the highest grade commercially available.

\section{Isolation of platelets.}

Washed human platelets were obtained from fireshly drawn blood by differential centrifugation as described before [50]. Platelets were finally resuspended in a Hepes buffer composed of $136 \mathrm{mM} \mathrm{NaCl}, 27 \mathrm{mM} \mathrm{KCl}, 2 \mathrm{mM}$ $\mathrm{MgCl}_{2}, 10 \mathrm{mM}$ Hepes (4-(2-hydroxyethy)-1-piperaxineethanesulfonic acid), $100 \mathrm{nM} 2 \mathrm{~K} 36374,5 \mathrm{mM}$ glucose, pH 75.

\section{Preparation of DLPC vesicles.}

DLPC was dried from a solution in chlloroform/methanol $(1 / 2, w / v)$ by a stream of nitrogen. Hepes buffer was added and after sonication for $15 \mathrm{~min}$, large particles were removed by centrifugation at $50000 \times \mathrm{g}$ for $30 \mathrm{~min}$. The phospholipid concentration was determined according to Bötiger et.al. [60].

\section{Incubation of platelets with DLPC wesicles.}

One volume of DLPC vesicles was added to nine volumes of a suspension containing $510^{8}$ platelets/ml. The final concentration DLPC was $100 \mu \mathrm{M}$. Incubation was carried out at $22^{\circ} \mathrm{C}$ whout stirning. To determine the amount of platelet lysis, lactate dehydrogenase activily present in a $30000 \times \mathrm{g}$ supernatant was measured according to Wroblewski and La Due [710]. 


\section{Isolation and characterization of platelet spicules.}

Platellet spicules were isolated by centrifugation of the total platelet/freside incubation at $800 \mathrm{mg}$ for $10 \mathrm{~min}$. The resuspended $800 \mathrm{~kg}$ pellet is further referred to as remnant platelets. The supernatant, which contains spicules, DLPC vesicles and some residual platelets, was centrifuged at $30000 \mathrm{~kg}$ for 30 min to separate spicules from DLPC vesicles. The pellet containing the spicules was resuspended in $1 \mathrm{ml}$ Hepes buffer and layered on top of $15 \mathrm{ml}$ $30 \%$ Percoll preparaed in the same buffer. After another 30 min centrifugation at $30000 \times \mathrm{g}$ a single band of pure platelet spicules was isolated. Percoll was removed afterwards by three additional washings in Hepes buffer. Prothrombinase activity was measured as described below. Phospholipids analysis was carried out after extraction according to Bligh and Dyer [61] and two-dimensional thin-layer chromatography as described previously [666].

Fatty acid composition of total platelet phospholipids tras analyzed by gas-liquid chromatography. From the ratio lauric acid over total fatty acid, the amount of exogenously incorporated DLPC could be calculated. Polyacrylamide gel electrophoresis was performed in the Laemmli system $[356]$, using $7.5 \%$ polyacrylamide slab gels with a $4 \%$ stacking gel. Samples for electrophoresis were dissolved in $2 \%$ SDS (w/v), 5\% -mercaptoethanol. $(\mathrm{v} / \mathrm{v}), 50 \mathrm{mM}$ Tris-HCl and $1 \mathrm{mM}$ EDTA, pH 8. Protein determination in the electrophoresis samples were done after 100-fold dilution using the methad of Lowry. Gels were stained with Coomassie Brilliant Blue for total protein. Glycoproteins were viswalized on nitrocellulose blots using peroxidase-conjugated lectins (wheat germ agglutinin or concanavalin A).

\section{Assay of prothrombinase activity.}

Purified coagulation factors $\mathrm{Xa}_{\mathrm{a}} \mathrm{Va}$, and prothrombin were prepared as described elsewhere [543a, 544]. Prothrombinase activity of either the total incubation mixture of platelets and DLPC vesicles or of the isolated spicules mas measured as follows: $312 \mu$ platelet or spicule suspension was incubated for $3 \mathrm{~min}$ in the presence of $13 \mu \mathrm{l} \mathrm{CaCl}$ (final concentration $3 \mathrm{mM}$ ). Subsequently $50 \mu$ lof a mixture of factors $\mathrm{Xa}$ and $\mathrm{Va}(30$ and $60 \mathrm{mM}$, respectively) was added 2 min later, followed by addition of $125 \mu \mathrm{l}$ prothrombin (16 $\mu \mathrm{M}$ in a buffer containing $50 \mathrm{mM}$ Tris, $175 \mathrm{mM} \mathrm{NaCl}, 14 \mathrm{mM} \mathrm{CaCl}$, and 05 $\mathrm{mg} / \mathrm{ml}$ human serum albumin, $\mathrm{pH} 7.9$ ). After $1 \mathrm{~min}$, prothrombin activation was arrested by transfer of a $25 \mu 1$ subsample into a cuvette contäining $1 \mathrm{~m} / 150 \mathrm{mM}$ Tris, 20 . mM EDTA, $175 \mathrm{mM} \mathrm{NaCl}$, pH 7.9. The amount of thrombis formed was calculated from the change in absorbance at $405 \mathrm{~nm}$ after addition of chromogenic substrate $S 2238$ to a finall concentration of $150 \mu \mathrm{M}$, using a calibration curve made with active site titrated thrombin.

\section{Electron microscopy.}

Platelet suspensions were fixed with glutaraldehyde (2.5\% in $100 \mathrm{mM}$ phosphate buffer, $\mathrm{pH} 7.3)$. The samples were washed and postfixed in osmium tetroxide ( $1 \%$ in the same phosphate buffer). Dehydration was carried out in a graded series of ethanol after which the preparations were embedded in Epon 812 . Uttrathin sections were stained with a saturated solution of uranylacetate and lead citrate $(0.25 \%$ w/v) adjusted to $\mathrm{pH} 12$ using $\mathrm{NaOH}$. Mierographs were taken with a Philips CM12 mieroscope. Isolated spicules were negatively stained with $2 \%$ uranylacetate on carbon-coated formwar-films.

\section{RESULTS}

Addition of DLPC vesicles to a platelet suspension results in a rapid increase of prothrombinase activity, which reaches a maximal value after approximately $45 \mathrm{~min}$ incubation (Fig. 1). The increased prothrombinase activity is not the result of exposure of the cytoplasmic side of the membrane due to cell rupture, since less than $3 \%$ of the cells were lysed after $60 \mathrm{~min}$ incubation. Addition of $100 \mathrm{nM} Z \mathrm{ZK}-36374$, a stable carbacyclin derivative of prostacyclin, which is a powerful inhibitor of platelet aggregation and release [27], did not prevent expression of procoagulant activity.

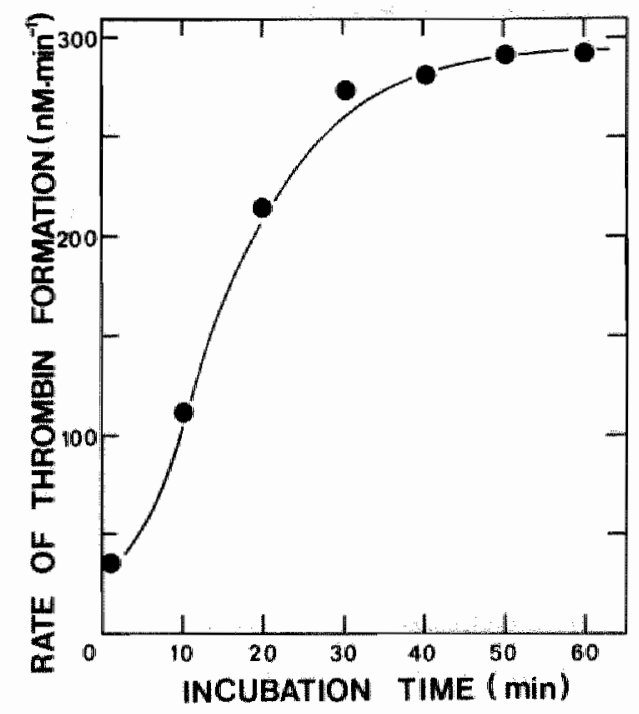

Fig. 1. Time dependent prothrombinase activity upon incubation of washed human platelets with DLPC vesicles. Samples were taken from the incubation mixture at different time intervals and dilluted with Hepes buffer (pH 7.5) for measuring prothrombinase activity. Platelet concentration in the prothrombinase assay was $1.210^{7} \mathrm{ml}^{-1}$. Prothrombinase activity is expressed as the rate of thrombin formation (nM per min). 
Significant morphological changes accompany the development of procoagulant activity in platelets incubated with DLPC vesicles (Fig. 2). Although it cannot be excluded that the process of spicule formation is not immediately arrested by addition of glutaraldehyde, the appearance of small vesicular structures, pinched off from the platelets, can allready be observed one minute after addition of DLPC vesicles. Apart from the formation of more spicules no further morphological changes are observed upon prolonged incubation up to 60 min. Isolation of these platelet spicules on Percoll, followed by negative staining, showed under the electron microscope spherical structures with diameters ranging from 100 to 150 nm.
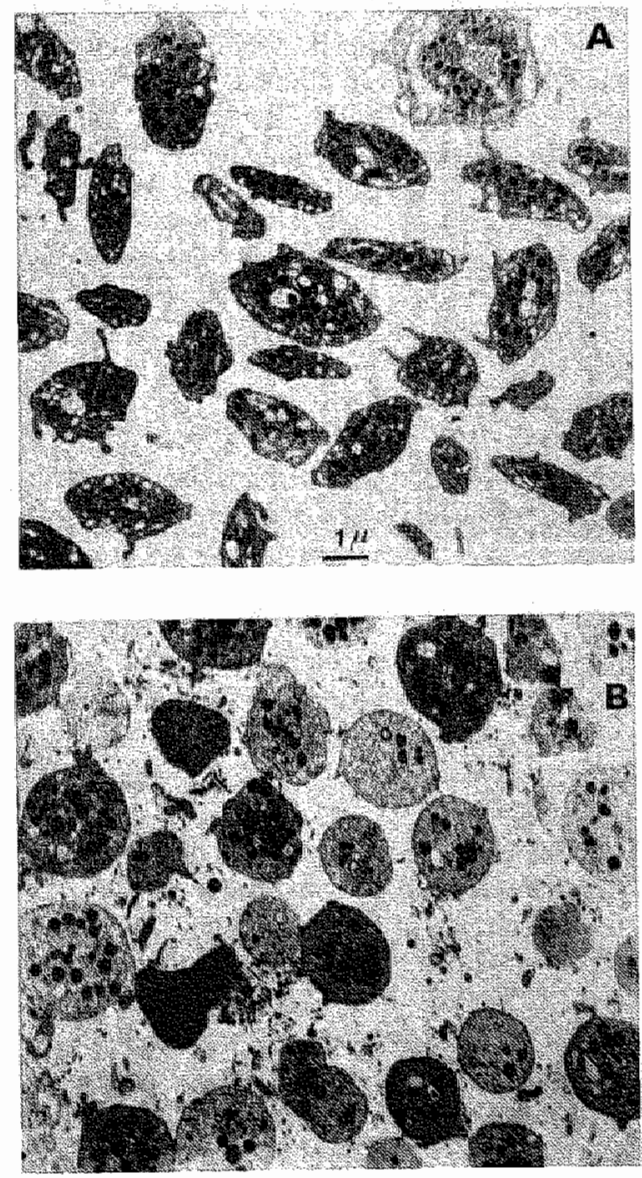

Fig. 2. Electron micrographs of control platelets (A) and platelets incubated with DLPC vesicles for $1 \mathrm{~min}$ (B).
Prothrombinase activities of isolated platelet spicules, control platelets and remnant cells are presented in Table $\mathrm{I}$. The prothrombinase activity of a cell or vesicle preparartion depends on the concentration as well as the composition of the phospholipids. To eliminate the effect of different phospholipid concentrations, prothrombinase activities were expressed per $\mu \mathrm{mol}$ phospholipid. Isolated platelet spicules exhibit a prothrombinase activity of 218 nmol thrombin/min per $\mu \mathrm{mol}$ phospholipid, which is a 30 -fold increase with respect to the activity of intact control platelets. Remnant platelets have a prothrombinase activity of 52 nmol thrombin/min per $\mu \mathrm{mol}$ phospholipid, which is also increased relative to control platelets though considerably less than observed for platelet spicules. Maximal prothrombinase activity of platelet remnants and spicules can be obtained after disruption of the membranes by sonication. As shown in Table I, the prothrombinase activities for the various sonicated preparations do not differ significantly when expressed per $\mu$ mol phospholipid.

The phospholipid composition of control platelets, platelet spicules and remnants are similar (Table II), although the amount of $\mathrm{PC}$ in the platelet spicules and remnants is increased due to the incorporation of exogenous DLPC (17.3 and $14.9 \%$, respectively). Therefore, it is concluded that the elevated prothrombinase activity of platelet spicules is not the result of an enrichment in procoagulant phospholipids, but must be due to increased exposure of PS at the surface of the spicules. Phospholipases, either alone or in combination with phospholipid transfer protein, have been used succesfully to localize phospholipids in the plasma membrane of various cells $[217,476]$.

TABLE I. Prothrombinase activities of control platelets, platelet spicules, and remnant platelets after incubation with DLPC vesicles.

\begin{tabular}{lcc} 
& Prothrombinase activity \\
\hline control platelets & 8 & $(1250)$ \\
platelet spicules & 219 & $(1442)$ \\
remnant platelets & 52 & $(1012)$ \\
\hline
\end{tabular}

Prothrombinase activities are expressed as nM thrombinfmin per $\mu$ mol phospholipid. Values in parenthesis are obtained after complete lysis by extensive sonication. 


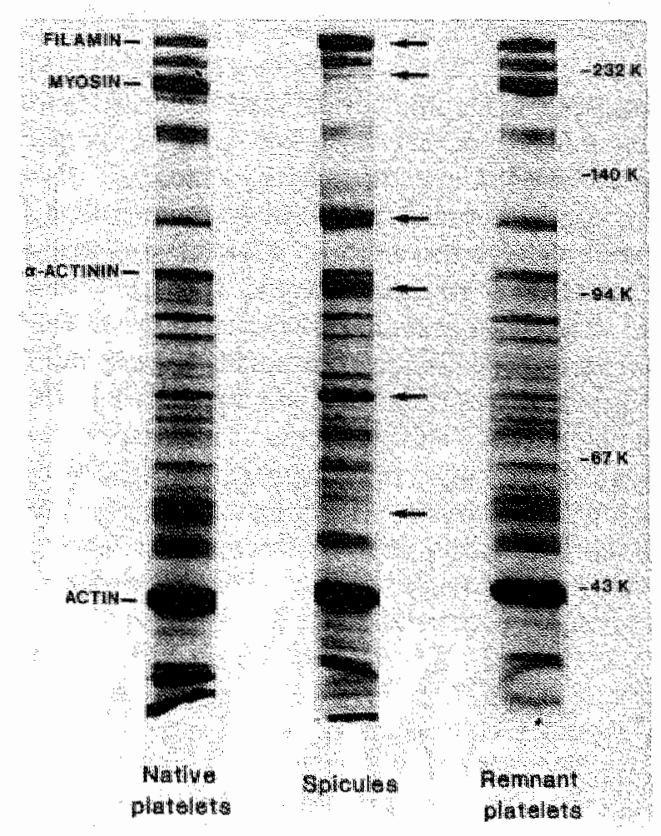

Fig. 3. SDS-polyacrylamide gell electrophoresis of control platelets, isolated DLPC induced platelet spicules, and remnant platelets after removal of platelet spicules and DLPC vesicles. Gels were stained with Coomassie Brilliant blue. Cytoskeletal proteins are indicated on the left and molecular weight standards on the right. The arrows identify major differences between the protein patterns. Spicules were isolated after 45 min incubation of platelets $\left(510^{8}\right.$ per ml) with DLPC vesicles $(100 \mu \mathrm{M})$, as described in Materials and Methods.
Attempts to apply this technique on isolated spicules resulted in virtually complete hydrolysis of all phospholipids and extensive lysis of the spicules as judged from the liberation of lactate dehydrogenase (data not showin). Presumably, the small size of the platelet spicules does not allow the use of these techniques, as was also found for spicules derived from sickle cells [217]. Therefore, the distribution of phospholipids over the spicule membrane remains uncertain.

Protein analysis of isolated spicules and control platelets reveals number of differences as illustrated in Fig. 3. The most conspicuous difference is the virtual absence in spicules of a band at $200 \mathrm{kDa}$, corresponding to the heavy chain of myosin. Furthermore, two increased bands at $125 \mathrm{kDa}$ and $98 \mathrm{kDa}$ are observed in the spicules. These bands likely represent increased amounts of glycoproteins $\mathrm{II}_{\mathrm{b}}$ and III $_{a}$ (see also Fig. 4). Finally, the spicules show a distinctly increased band at $76 \mathrm{kDa}$ and lack a band at $59 \mathrm{kDa}$, both of which have not been identified. Similar protein patterns were obtained by Kobayashi et.al. [330] for spicules isolated from rabbit platelets upon treatment with DLPC.

Advantage was taken of the presence of glycoproteins in the spicules to define the sidledness of the spicule membrane. Glycoproteins were detected on nitrocellulose blots stained with peroxidase-conjugated concanavalin $\mathrm{A}$ or wheat germ agglutinin before and after treatment of the spicules with a-chymotrypsin. As a control, intact platelets were treated and analysed in the same manner.

TABLE II. Phospholipid composition of control platelets, platelet spicules and remnant platelets after treatment with DLPC vesicles.

\begin{tabular}{lccc}
\hline phospholipid class & control platelets & platelet spicules & remnant platelets \\
\hline phosphatidylserine & $10.8 \pm 0.5$ & $8.5 \pm 0.5(10.3)$ & $9.2 \pm 1.1(10.9)$ \\
phosphratidylinositol & $3.1 \pm 1.3$ & $1.7 \pm 0.4(2.1)$ & $3.1 \pm 1.8(3.7)$ \\
phosphatidylethanolamine & $26.8 \pm 1.8$ & $19.7 \pm 0.9(23.8)$ & $21.3 \pm 1.0(25.1)$ \\
phosphatidylcholine & $39.8 \pm 1.6$ & $52.1 \pm 0.9(42.1)$ & $48.2 \pm 2.0(39.3)$ \\
sphingomyelin & $19.3 \pm 0.5$ & $18.0 \pm 0.8(21.8)$ & $17.8 \pm 0.6(21.0)$ \\
\hline
\end{tabular}

Data are expressed as mol\% of total phospholipid fraction (mean $\pm S . D, n=3$ ). The numbers in parenthesis represent moll\% of total phospholipids corrected for incorporated DLPC, which anourts 17.3 and $14.9 \%$ in the spicules and rem. nant platelets, respectively. 
Fig. 4A shows the concanavalin A-stained blots of platelets and spicules before and after treatment with chymotrypsin. Complete loss of the concanavalin A-binding sites of glycoproteins $\mathrm{I}_{b}$ and $\mathrm{II}_{a}$ was found after chymotrypsin treatment of both platelets and platelet spicules. A similar finding was obtained upon wheat germ agglutinin staining (Fig.4B), showing complete removal of the wheat germ agglutinin binding sites of glycoproteins $\mathrm{I}_{\mathfrak{a}}$ and $\mathrm{I}_{\mathrm{b}}$. Moreover, more than $97 \%$ of the sialic acid residues in the spicule preparation are removed upon chymotrypsin treatment. These findings demonstrate that, in comparison with the plasma membrane of control platelets, the spicule membranes are right-side-out with respect to the orientation of the major glycoproteins. In addition, Coomassie-stained gels of control platelets and platelet spicules before and after chymotrypsin treatment (Fig. 4C) reveal no detectable hydrolysis of the cytoskeletal proteins talin $(235 \mathrm{kDa})$, myosin $(200 \mathrm{kDa}), \alpha$-actinin $(105 \mathrm{kDa})$ and actin $(43 \mathrm{kDa})$, indicating that these proteins are indeed localized in the interior of the spicules. Moreover, this finding indicates that the spicule membrane and platelet plasma membrane remain intact after chymotrypsin treatment, in line with the fact that no lactate dehydrogenase was liberated from the spicules during the incubation. The small, but apparent degradation of filamin $(250 \mathrm{kDa})$ in both intact platelets and spicules upon chyomotrypsin treatment is rather unexpected and difficult to reconcile with the cytosolic localization of fillamin. It is possible, however, that an un-
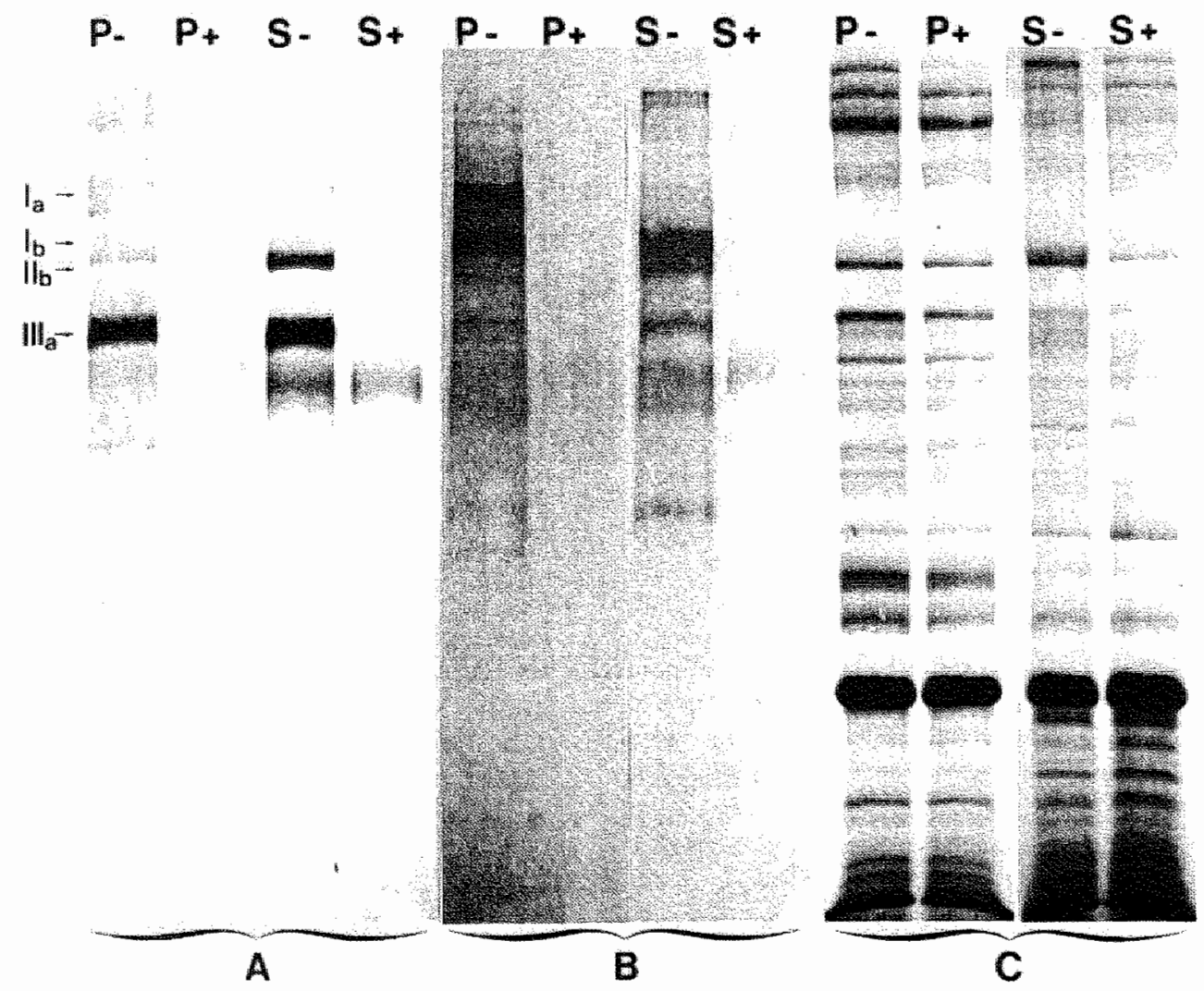

Fig. 4. SDS-polyacrylamide gel electrophoresis of control platelets (P) and isolated DLPC induced platelet spicules (S) before $(-)$ and after $(+)$ treatment with chymotrypsin. (A) Nitrocellulose blot stained with peroxidase-conjugated concianavalin A. (B) Nitrocellulose blot stained with peroxidase-conjugated wheat germ agglutinin. (C) Gel stained with Coomassie Brilliant blue. Major glycoproteins (GP) are indicated in the figure: $\mathrm{GPI}_{\mathrm{a}}$ at $150 \mathrm{kDa}, \mathrm{GPI}_{\mathrm{b}}$ at $132 \mathrm{kDa}$, GPII at $125 \mathrm{kDa}_{\text {, and }} \mathrm{GPIII}_{\mathrm{a}}$ at $98 \mathrm{kDa}$. 
known ectoprotein migrates at the same position in the gel as filamin.

Addivional information on the protein composition of the spicules comes from comparison of the concanavalin $A$ - and wheat germ agglutinin-stained protein patterns (Figs. $4 \mathrm{~A}$ and 4B). Platelet spicules seem to be enriched in glycoproteins $\Pi_{b}$ and $\Pi_{a}$, although this could partially be a reflection of the increased ratio of membrane proteins over total protein, due to the small size of the spicules. Furthermore, from the patterns stained with wheat germ agglutinin it appears that the spicules lack a glycoprotein at approximately $160 \mathrm{kDa}$, which is possibly identical to glycoprotein $I_{a}$. Preliminary studies using two-dimensional gel electrophoresis have confirmed the lack of glycoprotein $\mathrm{I}_{\mathrm{a}}$ and showed that also glycoprotein $\mathrm{II}_{\mathrm{a}}$ is virtually absent in the spicules (Clemetson, KJ., personal communication).

To summarize the differences in protein composition between spicules and remnant platelets, their selective enrichment in cytoskeletal proteins is shown in Fig. 5 , in relation to the process of spicule formation.

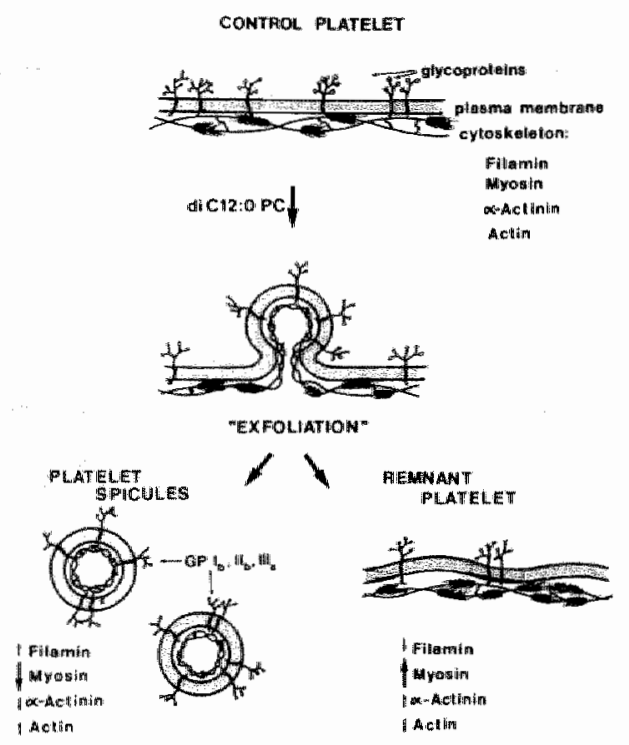

Fig. 5. Differences in protein composition between DLPC-induced patelet spicules and remnant platelets in relation to the process of spicule formation. Increase or decrease in the content of cytorkeletal proteins relative to control platelets is indicated by upward and downward arrows, respectively.

\section{DISCUSSION}

In the present paper we demonstrate that the prothrombinase activity of a suspension of human platelets rapidly increases upon incubation with DLPC vesicles. The increased prothrombinase activity predominantly resides on the surface of small vesicular structures which can be separated from remnant platelets and DLPC vesicles on a Percoll gradient. Since the ability of a membrane surface to enhance prothrombinase activity is determined by the presence of negatively charged phospholipids, in particular PS [50, 543a], it is concluded that spicule formation is accompanied by an increased exposure of PS to the external medium containing the coagulation factors.

The increased exposure of PS on the spicule surface is not due to an inversion process of the platelet plasma membranes, which would lead to exposure of PS present in the cytoplasmic leaflet of the plasma nembrane. This is based on the following observations: (1) no appreciable lysis accompanies the process of spicule formation, and (2) spicule membranes are right-side-out, as judged from the orientation of the membrane (sialo-)glycoproteins and the localization of cytoskeletal proteins within the spicules. Furthermore, the increased exposure of PS is not the result of a selective enrichment of this phospholipid, since, apart from the amount of incorporated DLPC, no significant change in composition of the other lipids could be observed in the isolated spicules. The small increase in PS content of the isolated spicules in comparison to control platelets, as reported by Kobayashi et.al. [330], might be due to a different isolation procedure of the spicules or merely reflects a species difference (rabbit vs. human platelets). Finally, the increased exposure of PS is not caused by a platelet release reaction, because this effectively inhibited by the presence of a stable prostacyclin analogue. It is therefore concluded that the shedding process, induced by DLPC, causes a spontaneous loss of the asymmetric distribution of PS over the platelet plasma nembrane.

It is presently unknown whether the increased exposure of PS induced by DLPC occurs during the actual shedding process or is the result of a redistribution of this lipid in the spicule membranes, after their detachment from the main cell body. It might be speculated, 
however, that the exfoliation of the plasma membrane as such, which preceeds the formation of membrane vesicles, is sufficient to cause at least some loss of transbilayer asymmetry of PS, since the remnant cells also exhibit an increased prothrombinase activity.

The rate at which the prothrombinase activity of platelets increases upon incubation with DLPC, suggests a fast redistribution of PS in the platelet or spicule membrane. A rapid loss of transbilayer asymmetry of PS is likely to require both enhancement of transbilayer movement and a loss of stabilizing forces, as for instance provided by cytoskeletal interactions. An increased transbilayer movement could be facilitated by the relatively large amount of incorporated DLPC (approx. 20\%) in the membranes. The presence of these short fatty acyll chain phospholipids might be responsible for disturbances of the lipid bilayer structure, at which transbillayer movement of phospholipids is facilitated.

Extensive studies on erythrocytes have led to the concept that the asymmetric distribution of $\mathrm{PS}$ in the red cell membrane is maintained by two independent mechanisms, i.e. (1) its ATP dependent translocation towards the inner monolayer $[144,643,721]$, and (2) its interaction with the membrane skeleton, in particular with spectrin $[217,256,259,703]$. Recently, the existence of both mechanisms has been demonstrated in a single study by Middelkoop et.al. [420].

The absence of myosin in the platelet spicules suggests an alteration of the molecular architecture of the cytoskeleton. In analogy to the model which explains the asymmetric distribution of PS in the red cell membrane, we propose that a different cytoskeletal organization in the platelet spicules is responsible for a loss of interaction between PS and cytoskeletal proteins. As a result, PS (partially) randomizes over both membrane leaflets. The involvement of a membrane cytoskeletion interaction in regulating transbilayer phospholipid asymmetry in the platelet plasma membrane is supported by previous studies $[124,663,664]$ in which it was demonstrated that upon stimulation with various agonists, the extent of PS exposure at the outer surface of the platelet is related to the extent of degradation of cytoskeletal proteins (including myosin) by callpain, the endogenous $\mathrm{Ca}^{2+}$-dependent protease. In this respect it is of interest to note that two different myosin isoenzymes have been demonstrated in platelets [492]: a soluble form which represents cytosolic myosin and a slightly different form, which comprises about $25 \%$ of the totall myosin and appears to be strongly bound to the membrane since it could not be removed by repeated washings.

An asymmetric distribution of phospholipids over the platelet plasma membrane is likely to involve an energy requiring process. It may be speculated that the myosin ATPase activity in platelets could play an active role, either via a direct interaction with amino phospholipids or through maitenance of a particular cytoskeletal conformation, which would be required for an interaction with specific phospholipids. Experiments determining the effect of energy depletion on the localization of PS in the platelet plasma membrane are now in progress in our laboratory.

\section{ACKNOWLEDGEMENTS}

Mr. P.H.H. Bomans is gratefully acknowledged for preparing the electron micrographs, and Mrs. M Feijge and Mrs. W.M.A. Linskens for excellent technical assistance. 

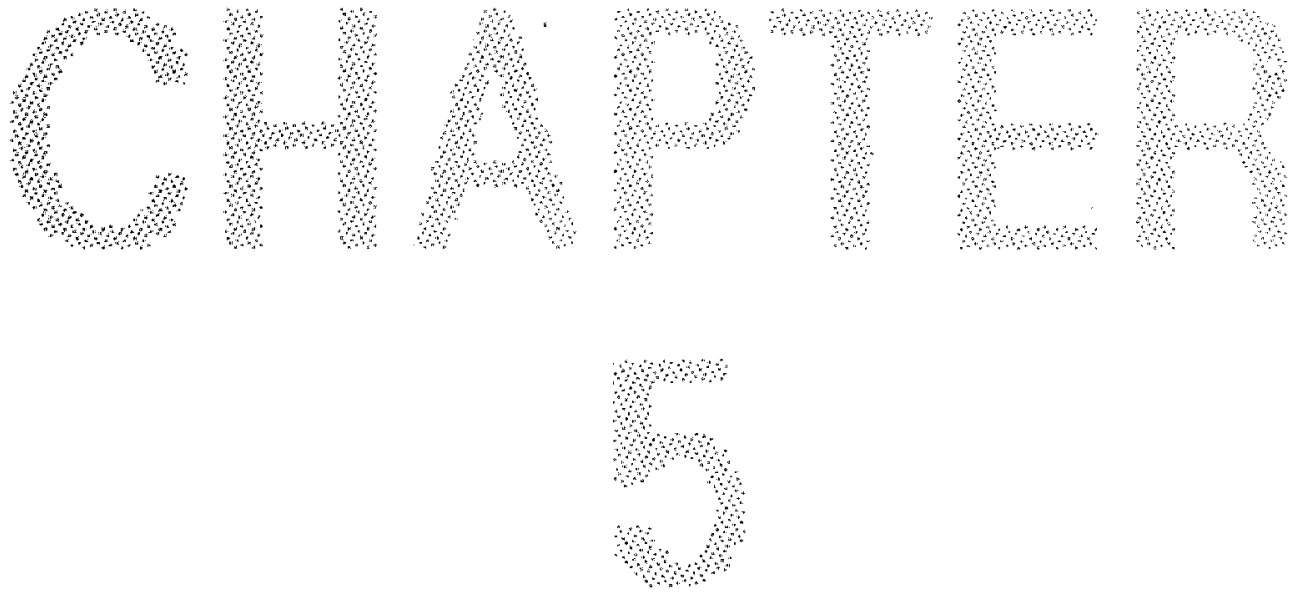


\title{
Transbilayer asymmetry of membrane fluidity and cholesterol in unstimulated platelets.
}

\begin{abstract}
The existence of a transmembrane fluidity gradient in human platelets was investigated employing trimethylammonium-diphenylhexatriene (TMA-DPH) to probe membrane fluidity and ultrasensitive time-resolved fluorimetry to monitor and analyze the fluorescence lifetime and motional properties of TMA-DPH. Lifetime analysis allowed direct measurement of the amount of TMA-DPH left in aqueous solution. The motional properties of TMA-DPH are represented by steady-state and time-resolved fluorescence anisotropy , and further detailed by structural order parameter and rotational dimusion coefficient. Both by fluorescence intensity and lifetime measurements, it was found that TMA-DPH gradually penetrates the platelet interior, on a time scale of several hours at $37^{\circ} \mathrm{C}$. During penetration of TMA-DPH into platelets, fluorescence lifetime, steady-state fuorescence anisotropy, and structural order parameter decreased, and rates of rotational movement increased dramatically. These changes in fuorescence lifetime and motional properties of TMA-DPH during its penetration into unstimulated platelets indicate that the outer leaflet of the plasma membrane is much less fuid than intracellular membranes and the cytosolic leaflet of the plasma membrane. To designate the membrane components responsible for the observed transmembrane fluidity gradient, the average nuorescence lifetime and motional properties of TMA-DPH in various membrane systems were investigated. These membrane systems include: sonicated platelets, platelet lipid vesicles, and phosphatidylcholine vesicles with and without either sphingomyelin, phosphatidylethanolamine, phosphatidylserine or cholesterol as additional component. From these reference membrane systems it is clear that cholesterol is the major determinant of membrane fluidity as monitored by TMA-DPH. In addition, sphingomyelin, acyl chain unsaturation and membrane proteins also influence membrane fuidity though only moderately as compared to cholesterol. In order to quantitate the contribution of cholesterol to the fluorescence lifetime and motional properties of TMA-DPH in membranes, dose-response relationships were determined. From these relationships, from the properties of TMA-DPH in the outer leaflet of the platelet plasma membrane, and from the relative contributions of the various membrane components to these properties, the cholesterol content of the outer monolayer is determined to yield a cholesterol/phospholipid ratio of 1.0. Assuming a cholesterol/phospholipid ratio of 0.7 for the whole platelet plasma membrane, the cholesterol/phospholipid ratio of the inner leaflet of the plasma membrane would amount to 0.4 . The observed transmembrane fluidity gradient is explained in terms of the heterogenous distribution of lipids throughout the human platelet.
\end{abstract}




\section{INTRODUCTION}

The lipids of the platelet plasma membrane are involved in various processes of platelet activation. At the cytosollic side they undergo fast membrane fusion during exacytosis [735], and supply precursors for second messengers like the polyphosphoinositides $[397,461]$ and esterified arachidonic acid [96, 152]. At the extracellular side, they are responsible for the platelet procoagulant activity, after transbilayer redistribution $[54,733]$.

In coherence with this functional asymmetry, the lipids of the platelet plasma membrane display a distributional asymmetry: the cytosolic leaflet is enriched with the aminophospholipids (PE, PS), while the cholinephospholipids ( $\mathrm{PC}, \mathrm{SphM})$ are predominantly present in the outer monolayer of the platelet plasma membrane [98, 494, 569]. Moreover, the acyl-chain composition of phospholipids also differs between the two leaflets of the platelet plasma membrane: arachidonic acid is predominantly present in the cytosolic leaflet [494], fulfilling the needs for prostaglandin synthesis.

A neglected aspect of phospholipid transbilayer asymmetry in the platelet plasma membrane, is the possibility of an associated difference in fluidity between the two bilayer halves [577]. Such a fluidity gradient is found in the erythrocyte $[163,428,587,634,703]$, the outer leaflet being more rigid than the inner leaflet. Considering the similarity in phospholipid transbilayer asymmetry between platelets and erythrocytes $[154,476,736]$, it can be speculated that the inner monolayer of the platelet plasma membrane will be more fluid than the outer monolayer. This would also be predicted from the rigidifying effect of SphM [62], and the fluidizing effect of unsaturated acylchains on lipid order [621].

The possibility of a fluidity gradient across the platelet plasma membrane was investigated

\section{ABBREVIATIONS}

PE: phosphatidylethandamine, PS: phosphatidylserine, PC: phosphatidylcholine, SphM: sphingomyelin, TMADPH: trimethylammonium-diphenythexatriene, C/P ratio: cholesterol/phospholipld ratio, IF: fluorescence intensity, TNP-Gly: trinitrophenyl-glycine, TNP-Glu: trinitrophery/-glutamate, TNP L Lys: trinitrophenyl- -ysine. employing the fluorescent membrane probe TMA-DPH. This probe seemed most suitable for studying transbilayer asymmetry of membrane fluidity, because of its particular properties. Addition of TMA-DPH to a cell suspension results in immediate labeling of the outer monolayer of the plasma membrane [345, 348]. However, most of TMA-DPH will remain in aqueous solution, due to a partition equilibrium [347], where it is hardly fluorescent [519]. Transbilayer movement of TMA-DPH [137] will result in a gradual labeling of intracellular compartments [347, 348]. Thus, the rationale has been that a fluidity gradient in the platelet plasma membrane would be revealed by a time-dependent change in dynamic fluorescence properties of TMA-DPH. An important advantage of this approach is the use of intact cells, since disruption of the interaction between cytoskeleton and membrane could result in loss of transmembrane fluidity gradient $[634,703]$. In addition, TMA-DPH is not cytotoxic $[345,347]$, allowing measurements in viable cells for prolonged periods.

Ultrasensitive time-resolved fluorescence depolarization was applied to monitor the changes in fluorescence properties of TMADPH. This technique has proven its versatility in lipid bilayers $[47,320]$ and biomembranes [12, $32,286,320,487,620]$, and provides a more detailed description of motional characteristics than fluorescence depolarization. Simultaneously, information about the fluorescence lifetime of TMA-DPH is abtained, which is an indicator for the polarity of the environment of TMA-DPH $[519,617,618]$. Interpretation of fluorescence anisotropy decays is dependent on the model describing the motion of the probe in the membrane [320]. In this respect, the use of TMA-DPH has an additional advantage: owing to its unimodal distribution in lipid bilayers parallel to the acyl-chains [617], its motional properties are adequately described by several diffusion models, like the general diffusion model [9] and the "wobbling-in-cone" model $[9,322]$. The use of time-resolved fluorescence depolarization raises the opportunity to discriminate between discrete probe populations [487, 670], and thus allows determination of the relative amount of TMA-DPH in aqueous solution.

Using the methodological approach outlined above, it will be shown that gradual penetration of human platelets by TMA-DPH is 
accompanied by dramatic changes in fuorescence properties of TMA-DPH, indicative of a significant difference in fluidity between the outer and inner monolayer of the platelet plasma membrane. In an attempt to designate the factor(s) responsible for the fluidity gradient, the effect of the major components of the platelet plasma membrane on the fluorescence properties of TMA-DPH in model systems was determined. From these results it is concluded that the transbilayer asymmetry of membrane fluidity can not only be explained by differences in acyl-chain unsaturation and SphM content, but also implicates an asymmetric transbilayer distribution of cholesterol. Based on a detailed investigation of the effects of cholesterol on the fluorescence properties of TMA-DPH and data from literature, it is proposed that the outer and inner leaflet of the platelet plasma membrane have a C/P ratio of 1.0 and 0.4 , respectively. The observed transmembrane fluidity gradient will be discussed in terms of the heterogenous distribution of lipids throughout the human platelet, and its physiological implications will be addressed.

\section{MATERIALS AND METHODS}

TMA-DPH was from Molecular Probes Inc. (Plano TX, USA). Cholesterol, PC and PE from egg-yolk, and SphM and PS from bovine brain were from Sigma (ST Louis MO, USA). All lipids were used without further purification, except for $\mathrm{PS}_{*}$ which was furhter purified by $\mathrm{CM}$-cellulose column chromatography as described [126]. Purity of lipids was confirmed by thim-layer chromatography. All other chemicalls were of the highest grade: commercially available.

\section{platelets:}

Human platelets were isolated, as outlined previously [51], by differential centrifugation from fresh blood drawn from bealthy volunteers who received no medication. Blood was anticoagulated with acid-citrate-dextrose. Platelets were washed in an isotonic buffer at pH 6.6, containing $10 \mathrm{miM}$ Hepes (4-(2hydroxyethyl)-1-piperazineethamesulfonic acid), $136 \mathrm{mM} \mathrm{NaCl}, 27 \mathrm{mM} \mathrm{KCl}, 2 \mathrm{mM}$ $\mathrm{MgCl}_{2}, 5 \mathrm{mM}$ glucose (Hepes buffer) in the presence of $0,05 \%$ fatty acid-free human scrum albumin. They were finally resuspended at a concentration of $210^{8} \mathrm{per} \mathrm{ml}$ in Hepes buffer pH 7.4 without albumin. Platelet concentration was inferred from their absorption at $405 \mathrm{~mm}$ using a calibration curve determined with a Coulter Counter. Platelet tysis was determined by assaying lactate debydrogenase ( $E C 1.1 .127$ ) in a $12000 x g$ supernatant according to Wroblewsiki and La Due [710].
Total platelet membranes were preparted by sonication in the presence of 1 mM EGTA Ror 5 min with 10 sec in. tervals, in a direct-probe sonicator. (MSE) at an arnplitude of $12 \mathrm{\mu m}$. Totial platelet lipids were extracter acconding to Bligh and Dyer 161$]$, from platelets pooled from 8 donors.

\section{Vesicles}

Small unilamellar vesicles were prepared by sonication of bydrated lipids. Lipids were stored in chloroform/meth anol $(1 / 1$, by vol $)$ at $-20^{\circ} \mathrm{C}$ under $\mathrm{N}_{2}$. Aliquots of lipid sol utions were evaporated to dryness under a stream of $\mathrm{N}_{2}$. Dry lipids were hydrated by addition of Heppes bufer (pHI 7.4, without albumin), which was degassed with $\mathrm{N}_{2}$. Vesicles were prepared by sonucation for 5 min above their transition temperature, with an amplitude of $14 \mu \mathrm{m}$. Cholesterol containing vesicles were sonicated for 10 min. After sonication vesicles were centrifuged at $14.000 \mathrm{~g}$ for 5 min. The final phospholipid concentration was deter mined according to Bötcher etal. [66], and varied between 2 and $5 \mathrm{mM}$. Cholestarol conostration was determined enzymatically by the chotesterol-oxidase assay, using a commercial kit (Sigma, StLonis MO, USA).

\section{Fluorescence measurements}

TMA-DPH was dissolved in $\mathrm{N}_{x} \mathrm{~N}$-dimethylformanide at concentration of $5 \mathrm{mM}$ and stoned at $-20^{\circ} \mathrm{C}$ in the dark. For every experiment an aliquot from this stock solution was diluted 10 times in Hepes buffer pH 7.4 to obtain a temporary stock solution, which was found to be stable for at least 5 hours. Washed human platelets were diluted into a $3 \mathrm{ml}$ quartz cuvatte with $\mathrm{Hepes}$ buffer $\mathrm{pH}$ 7.4 to a concentration of $2110^{7}$ per $\mathrm{ml}$ and labeled at $37 \mathrm{C}$ with 1 UM TMLA-DPH, added from the diluted stock solution under continuous stirring. Using this double ditution procedure, the final concentration of $\mathrm{N}, \mathrm{N}$-dimethylformamide in the cuwette could be kept very low $(0.02$ vol\%). After addition of TMA-DPH, platelets were kept in the dark at $37^{\circ} \mathrm{C}$ without stirring, and at regular intervals fuorescence properties of TMA-DPH were measured. Sonicated vesicles wene employed at a concentration between 20 and $50 \mu M_{4}$ under the same conditions as platelets. Preparation of TNP-derivatives of Lysine and Glutamate was based on the prowedure of Snyder and Sobocinsky [606], and was performed by reaction of $100 \mathrm{mM}$ trinutrobenzenesulfontic acid with $110 \mathrm{mM}$ Iysine or glutamate at $\mathrm{pH} 9.5$ in a mixture of water and dimethylsulfoxide (2/1 by vol.). Both TNP-derivatives exhibilted an idlentical absorption maximum at $420 \mathrm{~nm}$.

Fluorescence intensities (F) were recorded at $37 \mathrm{C}^{\circ}$ under continuous stirring ( 300 rev/min) with an SLM Aminco SPP-SOOC flworimeter equipped with a lighttight injection port and a stirring device (Hellma). Excitation and emiscion wavelengths were set at 365 and $450 \mathrm{~nm}$ with 5 and $20 \mathrm{~nm}$ slits, respectively. F was corrected for light-scatter and backgound fuoresecence using unlabeled controlls:

Phase fluorimetry was performed on a SLM-4800 fuorimeter according to Prendergast etial. [S19], using a POPOP reference solution in order to remove any colordependent response of the detector [361]. Lifetimes from phase shifits $\left(\tau_{p}\right)$ were determined at al frequency of 30 
Whit, by a crosecorrellation method ussing the computer program supplied by the manufacturer. Excitathon was acoomplistied ustag a monochromatior sel at 360 nim $(0.5$ nm bandpass). Emmitaion was passed through two KV408 cul-of filters (Schow) before detection. Background Muorescence and light-scatter amounted to less than $1 \%$ of total intensity and were neglected. All measurements were performed at $37^{\circ} \mathrm{C}$ whoul stirning.

Fluonescence decriy and huorescence anisotropy decay were determined with a laser-pulsed single photon counting funorimeter, described in detall clsewhere $[284,285$, 286 , 6711. TMA-DPH was excited at $310 \mathrm{~nm}$ by a frequiency-doubled, synchronously pumped rhodanine 60 dye laser. Emission was monitored above $450 \mathrm{~nm}$ through a K45 band-pass filter (Balzers). All measurememts were performed at $37^{\circ} \mathrm{C}$ without stiring.

\section{Analysis of time-resolved data}

Data were analyzed by a reference convolution method described in detail by Vos et.al. [673]. Corrections for light-scatter and background fluorescence were routincly made by substraction of the recording of a control sample without TMA-DPH.

Total fluorescence, $s(\mathrm{~b})$, was reconstructed from the parallel, $I_{j}(t)$, and perpendicular, $I_{\perp}(t)$, polarized intensity components to yield the fluorescence lifetime compoments $\tau_{i}$ with fractional amplitude $\alpha_{j}$ :

$$
s(t)=I_{/ /}(t)+I_{\perp}(t)=\Sigma a_{i} e^{(t-t / T i)}
$$

Routinely a biexponential function was employed, generally used by others to describe the fuorescence decaly of TMA-DPH $[47,364,617,620]$. In addition, a tri- or tetraexponential function, fitting from lower channel numbers, was used to include the fluorescence lifetime of TMADPH in solution (fixed at $38 \mathrm{psec}$ ). The average fluorescence lifetime, $\langle\tau\rangle$, was calculated from the individual components from:

$$
<\tau>=\operatorname{Latri}^{2} / 2 \alpha i \pi
$$

The anisotropy tecay function, $r(t)$, was obtained by simultaneous fitting of $I_{/}(t)$ and $I_{\perp}(t)$ according 10 ;

$$
\begin{aligned}
& I_{/ /}(t)=s(t) / 3[1+2 r(t)] \\
& I_{\perp}(t)=s(t) / 3[1-r(t)]
\end{aligned}
$$

The anisotropy decay of TMA-DPH in membranes can be adlequately interpreted within the framework of the "wobbling-in-cone" model $[9,620]$, introduced by Kinosithit ettall. $[320,322]$ :

$$
r(t)=r_{10}+\left(r_{0}-r_{\infty}\right) e^{(-t / \phi)}
$$

where $m$ is the fundamental anisotropy, $r_{\infty}$ is the residual anisotropy at the end of the experiment (about $30 \mathrm{~ns}$ ), and $\phi$ is the apparent relaxation time.

It is generally recognized that "Aluidity" is not a welldefined parameter at the molecular level. fluidity in nembranes is an anisotropic property, determined by both static (range of movement) and dynamic (rate of mo- tion) components. Therefore, both components were derived separately. The static component is expressed by the second-rank order parameter, $S$, and obtained from:

$$
S=\sqrt{r_{0} / r_{0}}
$$

For comparison with various litterature values, the coneangle $\theta_{c}$ describing the range of acy-chain motion in the "wobbling-inimecone" model, is determined from:

$$
S=1 / 2^{*} \cos \theta c\left(1+\cos \theta_{c}\right)
$$

The dynamic component is expressed by the modelindependent diffusion constant for rotation around an axis perpendicular to the probe symmetry axis, $D_{1}$, and ewaluated from:

$$
\mathrm{D}_{\perp}=\left(\mathrm{rip}_{0}-\mathrm{r}_{\infty}\right) /(6 \mathrm{rop})
$$

assuming that the anisotropy decays exponentially to $\mathrm{r}$ [9].

To accommodate the frequent use of steady-state anisotropy, $r_{s}$ to describe changes, in fuidity, $I_{\delta}$ was also derived using the Perrin-equation:

$$
r_{i s}=r_{\phi 0}+\left(r_{0}-r_{\infty o}\right)(1+\pi / \phi)
$$

in which $\tau$ was substituted by the average fluorescence lifetime, $\langle\tau\rangle$, from a biexponential fit, which excludes any contribution of the ultrafast fluorescence of TMADPH in buffer. In addition, steady-state anisotropy can be expressed as the degree of polarization, $P$, related to $r_{s}$ :

$$
\mathbf{P}=3 \mathbf{r}_{\mathrm{s}} /\left(2+\mathbf{r}_{\mathrm{s}}\right)
$$

To eliminate the inaccuracy introduced by variations in $\mathrm{r} 0$, ro was substituted by the average- $\mathrm{r} \mathrm{m}_{\mathrm{n}}$ which was determined from experiments and which amounted to $0.318 \pm 0.008$ within a $1 \%$ confidence itterval. This value is lower than reported for glycerol as solvent (about 0.39 , $[47,519]$, as observed before $[620]$, and is similar as found by van Langen et.al. [364]. Note, however, that the experiments presented used excitation at $310 \mathrm{~nm}$, which was the highest wavelength possible in the current setup.

\section{RESULTS}

\section{Gradual penetration of TMA-DPH into un- stimulated platelets.}

Of first concern was to show that TMADPH, if continuously present, would indeed penetrate the platelet interior and on which time scale this process would take place. Since cellular penetration by TMA-DPH already has been suggested from fluorescence intensity (F) 
measurements [ 347,348$]$, the development of $F$ was monitored during prolonged incubation of washed human platelets with TMA-DPH. At the conditions employed, the majority of TMA-DPH is still in the aqueous solution (1 $\mu$ M TMA-DPH and $2: 10^{7}$ platelets per $\mathrm{ml}$; vide infra). Under these conditions $\mathrm{F}$ was linear with platelet concentration up to $10^{8}$ per ml. After the addition of TMA-DPH, F sharply increased and stabilized within $2 \mathrm{~min}$. At this moment only a fraction of all membranes is labeled, as will be shown later. Taking into account the rapid labeling kinetics, it appears that the outer monolayer is quickly saturated, in agreement with previous reports $[345,348]$. Thus, any further change in $F$ would indicate unlabeled membrane becoming availlable for labeling by TMADPH.

Incubation of unstimulated platelets with TMA-DPH showed that F slowly increased with a gradually declining slope (Fig. 1A). After 5 hours at $37^{\circ} \mathrm{C}$, a plateau level still was not reached, while $F$ was doubled with respect to the starting value. At this time platelet lysis was $6 \%$, measured as LDH leakage. Therefore, the increase in $\mathrm{F}$ cannot be due to platelet lysis. It is tempting to interpret the gradual increase in $F$ as penetration of TMA-DPH into the platelet interior. However, one can imagine that during prolonged incubation of platelets with TMA-

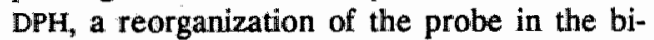
layer could give rise to increased incorporation of TMA-DPH. Alternatively, a slowly increasing interaction of TMA-DPH with hydrophobic domains of proteins, as illustrated by albumin [166], could also be responsible for the observed increase in F. To explore these possibilities, sonicated platelets were also incubated with $1 \mu \mathrm{M}$ TMA-DPH at $37^{\circ} \mathrm{C}$ (Fig. 1A). It was found that $F$ gradually decreased to reach a plateau level after 3 to 4 hours. Therefore, it is unlikely that a reorganization of TMA-DPH, without crossing the plasma membrane, is responsible for the observed increase in $\mathrm{F}$ during prolonged incubation of platelets with TMA-DPH.

Comparison of $\mathrm{F}$ from sonicated platelets and intact platelets $s_{\text {a }}$ few minutes after labeling, suggests that $F$ in intact platelets could rise less than a factor of three after disruption. This could be taken to indicate that more than onethird of total platelet lipids is present in the outer monolayer of the platelet plasma membrane. This value apparently deviates from reported estimates of about $25 \%[50,98,494]$. It should be emphasized, however, that quantitative interpretation of $\mathrm{F}$ is hampered by underlying changes in fluorescence lifetime, which will be described in a later section.

To obtain a more direct answer as to whether TMA-DPH penetrates unstimulated platelets, advantage was taken of a special property of TMA-DPH: the fast equilibrium between aqueous and lipid phase. At various time intervals during incubation of platelets with TMA-DPH, a subsample was dilluted hundredfold into buffer devoid of TMA-DPH under continuous monitoring of F. TMA-DPH present in the outer monolayer of the plasma membrane is expected to reeuilibrate rapidly with the buffer, while TMA-DPH within the platelet will reequilibrate much more slowly. It was found that F decreased abruptly upon dilution to reach a stable level within $30 \mathrm{sec}$, indicative for a new steady-state. It appeared that, during incubation of intact platelets with TMA-DPH for 4 hours, the residual $F$ after dilution increased tenfold (Fig. 1B), indicative for increased uptake of TMA-DPH by platelets. To exclude that increased binding to proteins in the outer

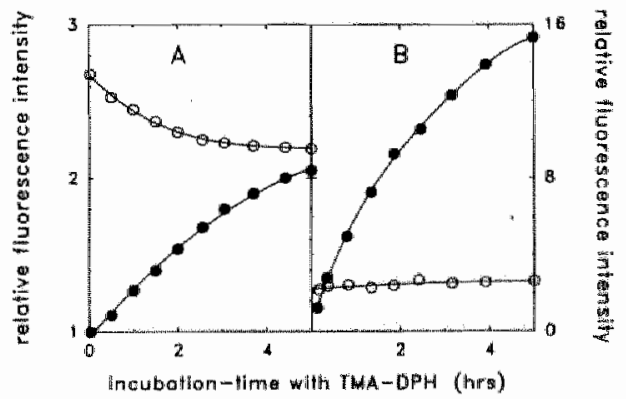

Fig. 1. Gradual penetration of TMA-DPH into unstimulated human platelets, as determined by fluorescence intensity measurements.

(A) Time-dependent changes in fluorescence intensity of $1 \mu$ M TMA-DPH incubated with unstimulated platelets $(\bullet)$ or sonicated platelets $(O)$ at a concentration of $210^{7}$ per $\mathrm{ml}$. (B) Residual fluorescence intensity of TMA-DPH in unstimulated platelets (O) or sonicated platelets $(0)$ after hundredfold dilution in Helpes buffer, $\mathrm{pH}$ 7.4. In this case, intact or sonicated washed human platelets were labeled with $5 \mu \mathrm{M}$ TMA-DPH at a concentration of $210^{8}$ per $\mathrm{ml}$, before dilution. $(A)$ and $(B)$; both platelet preparations represent equal amounts of platelets. All manipulations were carried out at $37 \mathrm{C}$. Further details are described in the methods. Data in both figures were obtained simultaneously during a representative experiment. 
monolayer of the plasma membrane is responsible for the increase in residual $F$ after dilution, sonicated platelets were used. It was found that during a period of 4 hours, sonicated platelets displayed a constant residual $F$ after dilu-
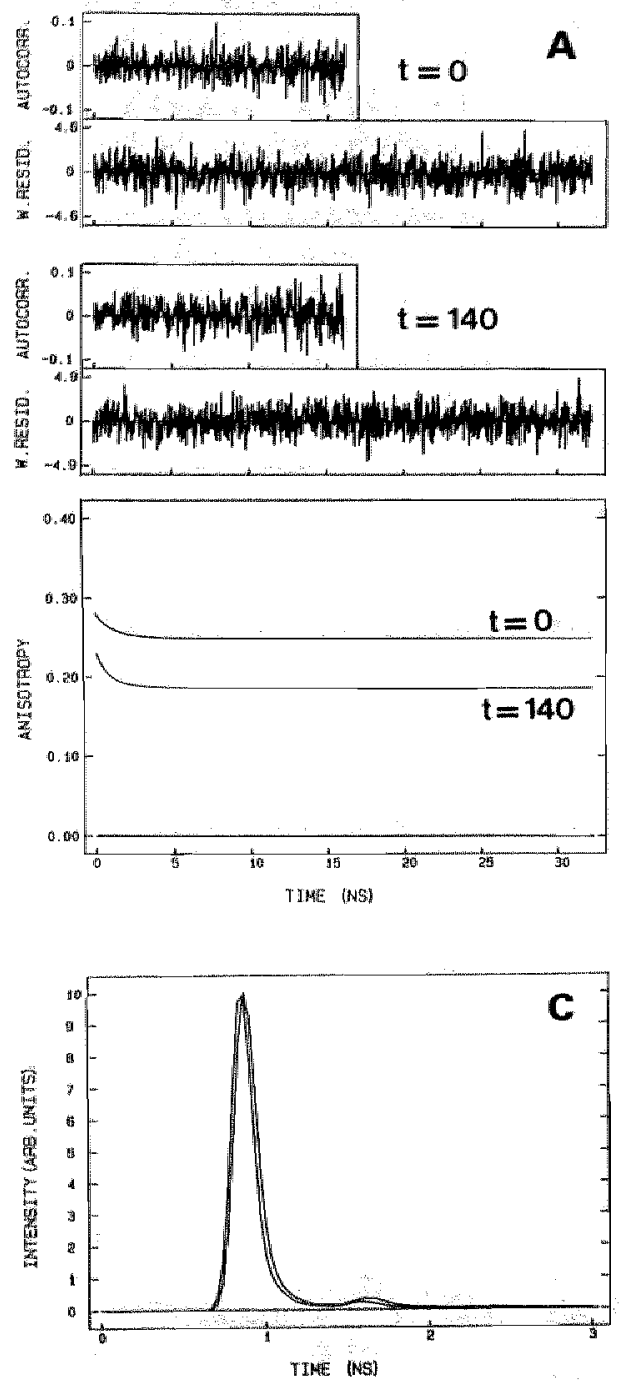

tion (Fig. 1B). Uptake of TMA-DPH could allso result from endocytosis. However, the increase in residual $F$ after dilution is accompanied by an increase in $F$, which cannot be explained by endocytosis per se. Therefore, it is concluded that TMA-DPH gradually penetrates platelet intracellular compartments as a result of slow transbilayer movement.

\section{Application of time-resolved fluorescence to monitor anisotropy and fuorescence lifetime of TMA-DPH.}

The fluorescence anisotropy decay of TMA-DPH in unstimulated platelets (Fig. 2A), as well as in all model membrane systems em-
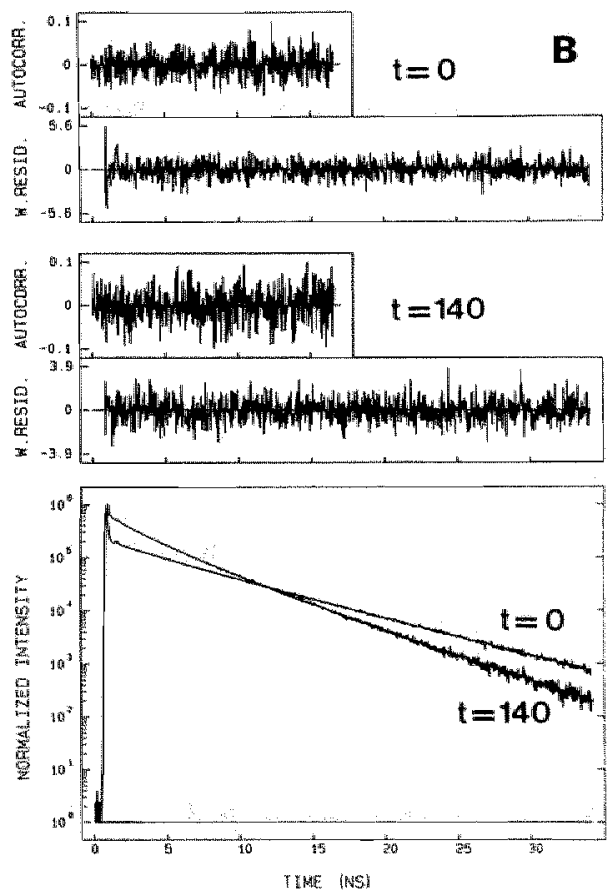

Fug. 2. Anisotropy decay (A) and intensity decay (B) analysis of TMA-DPH in unstimulated platelets at different incubation times (min). Data shown are representative for 5 separate measurements. The quality of the fit is indicated by the weighted residuals and the autocorrelation function of the weighted residuals on top of the curves. The corresponding fit parameters and derived fluorescence lifetime and anisotropy data are presented in table II and II, respectively. (A) To aid comparison, the noisy experimental anisotropy curves were discarded. The initial anisotropies of the fitted curves were lower than the true ones of ca. 0.31 , because the finite pulse width of the instrament leads to suppression of this value [486]. (B) Shown are the results of the tetra-exponential fit, found necessary when analysis was carried out right from the emission peak. (C) Fluorescence decay of $1 \mu \mathrm{M}$ TMA-DPH at $37^{\circ} \mathrm{C}$ in Hepes buffer pH 7.4 (curve shifted slightly to the right) , compared with the excitation pulse. The fluorescence lifetime was $38 \pm 3$ ps (mean \pm S.D. of three separate determinations). Experimental details are described in the methods. 
ployed, could be adequately described by a single exponential and a residual anisotropy (Table I). This means that the motion of TMADPH in lipid bilayers can be adequately described by the "wobbling-in-cone"' model [320], in agreement with previous findings $[9,620]$.

The fluorescence decay of TMA-DPH in platelets (Fig. 2B) as well as in model systems could be well represented by a short $\left(\tau_{1}\right)$ and a long $\left(\tau_{2}\right)$ lifetime component (Table II), in agreement with other observations [47, 137, $155,364,617,618,620$ ]. These two components are derived from a biexponential fit starting about $0.5 \mathrm{~ns}$ after the emission peak. Because most of TMA-DPH still must be present in aqueous solution, the fluorescence decay of TMA-DPH was measured in Hepes buffer only (Fig. 2C). The fluorescence decay could be described by a single lifetime of about $38 \mathrm{ps}$. The very short fluorescence lifetime of TMA-DPH in aqueous solution is in agreement with observations of Prendergast et.al. [519]. To incorporate the soluble pooll of TMA-DPH in the fluorescence lifetime analysis of TMA-DPH in membrane systems, the fluorescence decay was described by a triexponential function fitting right from the emission peak with $\tau_{1}$ fixed at $38 \mathrm{ps}$ (Table II). However, this approach did not result un good fits for all membrane systems, especially when the contribution of $\tau_{2}$ increased (note the statisticall criteria listed in Table II). Apparently, including short fluorescence lifetimes in the fit, an additional component, normally not seen by biexponential fits, is uncovered. Hence, the fluorescence decay of

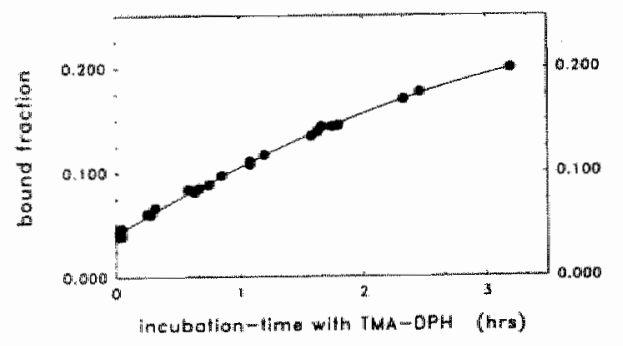

Fig. 3. Gradual penetration of TMA-DPH into unstimulated platelets, as determined from time-resolved fuorescence decay. The bound fraction is equivalent to the sum of the fractional amplitudes $c_{2}, \alpha_{3}$, and $\alpha_{4}$ from the tetraexponential decay fit, assuming only one water-soluble pool $\left(\alpha_{1}\right)$ described by $\tau_{1}$ (38 ps). Experimental details are described in the methodx. Data shown are pooled from 5 separate experiments.
TMA-DPH was analysed by a tetraexponential function, again with $\tau_{1}$ fuxed at 38 ps. This procedure resulted in excellent fits for all membrane systems (Table II). It is noted that the contribution of TMA-DPH in aqueous solution $\left(\alpha_{1}\right)$ is not significantly altered in the tetraexponential fit, supporting the view that the fluorescence decay of TMA-DPH in membranes actually is described by three components, the shortest of which escapes the normal biexponential fits starting some time after the emission peak. The average fluorescence lifetime of TMA-DPH in membranes will be represented by $\langle\pi\rangle$, in accordance with the usual presentation.

Changes in furorescence lifetime and anisotropy of TMA-DPH during penetration into unstimulated platelets.

Using the sensitivity of time-resolved fluorescence spectroscopy, it becomes possible to determine directly the fraction of TMA-DPH in solution. This is a great advantage, because it allows a full description of the distribution of TMA-DPH in lipid suspensions. This property was employed to reconsider the gradual penetration of TMA-DPH into unstimulated platelets. It was found that the fraction of TMA-DPH bound to platelets gradually increased on a time scale of several hours (Fig. 3), in agreement with the results from fluorescence intensity measurements. After 3 hours $_{i}$ the fraction of TMA-DPH bound to platelets was increased fourfold relative to the bound fraction immediately after labeling. This increase is significantly higher than obtained from fluorescence intensity measurements, which showed a less than twofold increase (Fig. 1A). This difference can be explained in terms of a decrease in fluorescence lifetime of TMA-DPH accompanying the increased penetration into platelets. In this way, $F$ is an underestimation of the bound fraction of TMA-DPH. In view of the amount of phospholipids found to be present in the outer monolayer of unstimulated platelets (approx. $25 \%)[50,98,494]$, an increase in bound fraction of four times relative to the bound fraction of TMA-DPH immediately after labeling, would be expected when platelets are fully equilibrated with TMA-DPH. Taking into account that 
TABLE I. Fuorescence anisotropy decay parameters of TMA-DPH in various membranes.

\begin{tabular}{|c|c|c|c|c|c|c|c|c|c|c|c|c|}
\hline $\begin{array}{l}\text { membrane } \\
\text { preparation }\end{array}$ & $\mathbf{r} 0$ & r & $\stackrel{\phi}{(\mathrm{nis})}$ & $x^{2}$ & DW & $Z_{p}$ & Zper & $r_{s}$ & $s$ & $\mathbf{P}$ & $\theta_{c}$ & $\underset{(\mathrm{MH} / \mathrm{L})}{\mathrm{DL}}$ \\
\hline $\begin{array}{l}\text { platelets } \\
(t=0 \text { min })\end{array}$ & $\begin{array}{l}321 \\
011\end{array}$ & .249 & $\begin{array}{r}1.08 \\
.14\end{array}$ & 1.08 & 188 & 218 & 259 & 258 & .884 & 343 & 229 & 34 \\
\hline $\begin{array}{l}\text { platelets } \\
(t=140 \mathrm{~min})\end{array}$ & $\begin{array}{l}323 \\
.018\end{array}$ & .187 & $\begin{array}{r}0.79 \\
.07\end{array}$ & 1.14 & 1.89 & 245 & 192 & .206 & .767 & 280 & 333 & 87 \\
\hline $\begin{array}{l}\text { sonicated } \\
\text { platelets }\end{array}$ & $\begin{array}{l}.329 \\
.006\end{array}$ & .222 & $\begin{array}{r}1.10 \\
.07\end{array}$ & 1.05 & 1.88 & 181 & 200 & .241 & .836 & 322 & 27.6 & 46 \\
\hline $\begin{array}{l}\text { platelet } \\
\text { lipid }\end{array}$ & $\begin{array}{l}.326 \\
.005\end{array}$ & $\begin{array}{l}.203 \\
.001\end{array}$ & $\begin{array}{r}1.15 \\
.05\end{array}$ & 1.09 & 1.85 & 205 & 172 & .227 & .800 & 306 & 30.7 & 52 \\
\hline PC & $\begin{array}{l}.311 \\
.004\end{array}$ & .001 & $\begin{array}{r}1.80 \\
.06\end{array}$ & 1.11 & 1.91 & 202 & 208 & .196 & .593 & .267 & 45.7 & 60 \\
\hline $\begin{array}{l}\text { PC/cholesterol } \\
\mathrm{C} / \mathrm{P}=1.4\end{array}$ & $\begin{array}{l}301 \\
.003\end{array}$ & $\begin{array}{l}.246 \\
.001\end{array}$ & $\begin{array}{r}2.16 \\
.19\end{array}$ & 0.99 & 1.92 & 214 & 228 & 264 & .879 & .350 & 23.5 & 18 \\
\hline $\begin{array}{l}\mathrm{PC} / \mathrm{PE} \\
(7 / 3)\end{array}$ & $\begin{array}{l}.289 \\
.005\end{array}$ & ${ }_{.112}^{.112}$ & $\begin{array}{r}2.36 \\
.13\end{array}$ & 1.25 & 2.00 & 236 & 217 & 212 & .593 & .288 & 45.7 & 46 \\
\hline $\begin{array}{l}\text { PC/SphM } \\
(7 / 3)\end{array}$ & $\begin{array}{l}314 \\
.004\end{array}$ & .136 & $\begin{array}{r}1.85 \\
.06\end{array}$ & 1.17 & 1.79 & 158 & 224 & .202 & .653 & .275 & 41.6 & 52 \\
\hline
\end{tabular}

Fluorescence anisotropy decay was analysed with a single exponential and a residual anisotropy, starting at channel 54 (emmision peak at channel 26 , total number of channelis is 1024 , time-equivalence per channel was $32.5 \mathrm{ps}$ ). Stamdard deviations of fitted parameters are indicated underneath. Fit quality can be judged from $x^{2}$, the Durbin-Watson parameter (DW), and the number of zero passages in the autocorrelation function in the parallel ( $Z_{p a r}$ ) and perpendicular (Zper) component. See also Fig.5. for graphical representation of the most relevant calculated parameters. All measurements were carried out at $37^{\circ} \mathrm{C}$ as described in the methods. Data shown are representative for at least 3 independent determinations.

Table III. Efrect of negatively and positively charged hydrophilic quenchers on the fluorescence lifetime of TMA-DPH in lipid vesicles.

\begin{tabular}{llll}
\hline Membrane preparation & quencher & before quencher & after quencher \\
PC-wesicles & TNP-Lys & $2.70(.04)$ & $1.53(.03)$ \\
& TNP-Glu & $2.72(.05)$ & $2.01(.04)$ \\
PC/PS-vesicles & TNP-Lys & $2.83(.04)$ & $2.25(.03)$ \\
$7 / 3$ molar ratio & TNP-Glu & $2.78(.06)$ & $2.61(.03)$
\end{tabular}

Quenching of TMA-DPH is monitored by a decrease in fluorescence lifetime as determined by phase fluorimetry ( $\tau_{\mathrm{p}}$ in $\mathrm{ms}$ ). Quenchers employed were the neutrally and negatively charged trinitrophenyl derivatives of lysine and glutamate $_{n}$ respectively. Vesicle concentration was $50 \mu \mathrm{M}$ phospholipid. Quencher concentration was $100 \mu \mathrm{M}$. Measurements were performed at $37^{\circ} \mathrm{C}$ as described in the methods. Data shown are the mean ( \pm s.d.) of 3 separate determinations. 
millimolar $\mathrm{Ca}^{2+}$ can decrease membrane binding of TMA-DPH (next chapter), and that the outer monolayer of the plasma membrane is more rigid than the inner (vide infra) and thus may linit uptake of TMA-DPH [720a], the ratio of TMA-DPH vs lipid may be lower in the outer monolayer than in intracellular membranes, resulting in a higher increase in membrane bound TMA-DPH upon penetration into platelets than can be expected on the basis of lipid ratios.

When fluorescence properties of TMADPH in unstimulated platelets are examined a few minutes after addition of TMA-DPH (Table $I$ and $I I, t=0 \mathrm{~min}$ ), an average fluorescence lifetime of $6.8 \pm 0.1 \mathrm{~ns}$ (biexponential fit) and a steady-state anisotropy of $0.260 \pm 0.005$ are abserved (mean \pm s.d., $n=5$ ). The fluores- cence lifetime for TMA-DPH in platelets is similar as reported by Kubina etal. [345]. The anisotropy is close to that reported by Kubina et.al. [345] and Donner \& Stolz [166], who found values of 0.275 and 0.267 , respectively. The tetraexponential fluorescence decay (Table 1) shows that immediately after addition of 1 $M$ TMA-DPH to $210^{7}$ platelets per $\mathrm{ml}$, about 96\% of total TMA-DPH is still present in the buffer. Assuming that under these conditions total platelet phospholipids amount to $8 \mu \mathrm{M}$, and that $25 \%$ of these lipids are in the outer monolayer, it can be estimated that the TMADPH/phospholipid ratio in the outer monolayer is about $1 / 50$.

Using a membrane probe at high probelipid ratios, there is a chance that the observed

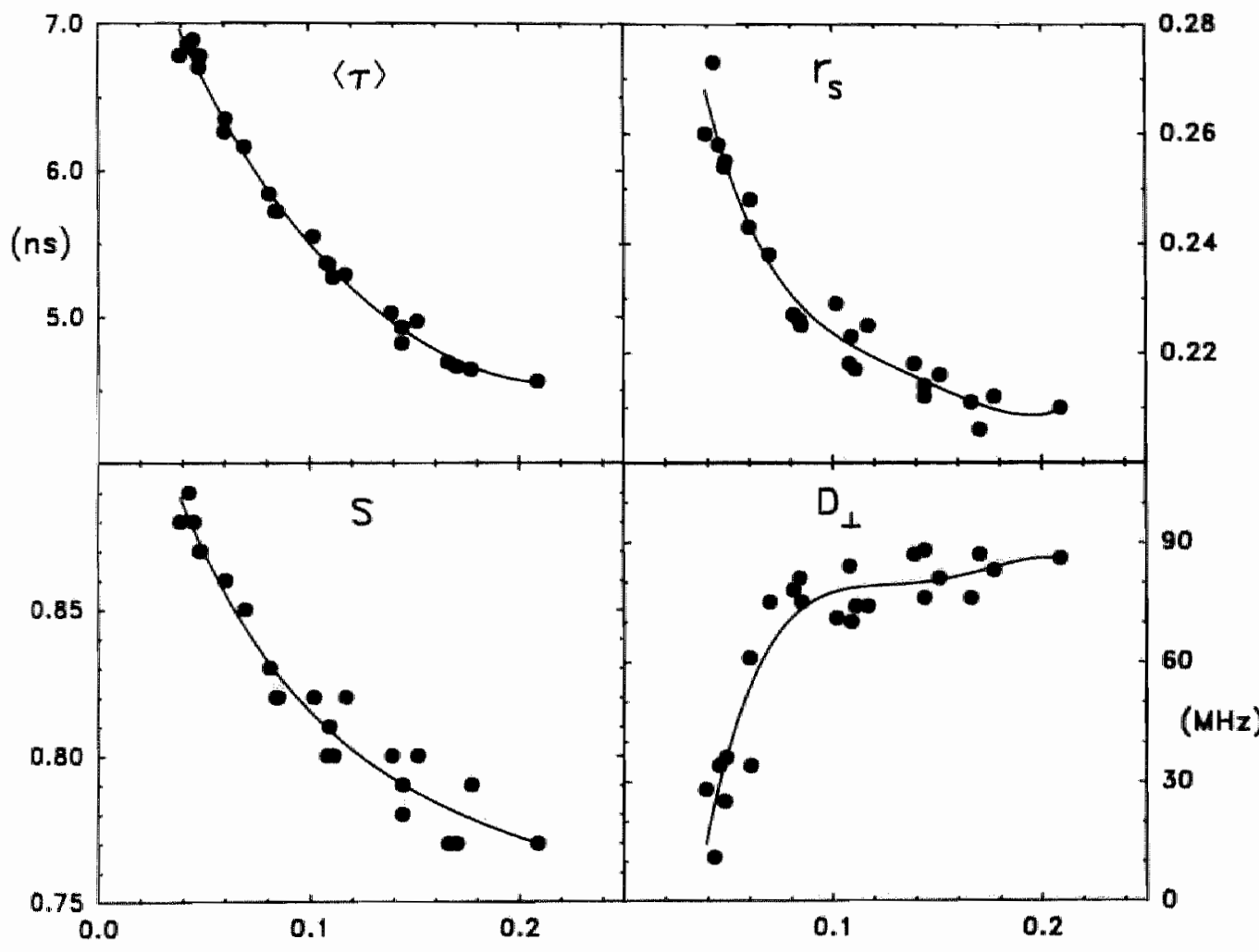

bound fraction of TMA-DPH

Fig. 4. Changes in fuorescence lifetime and motional properties of TMA-DPH accompanying its gradual penetration into unstimulated platelets. Presented are the average fluorescence liftime, $\langle\tau\rangle$, the steady-state anisotropy, $r_{S-}$ the structural order parameter, $S$, and the rotational diffusion constant, $D$ L. All parameters are plotted wersus the fraction of TMA-DPH bound to unstimulated platelets at $37^{\circ} \mathrm{C}$. Experimental details are des cribed in the methods. Datla shown are pooled from 5 separate experiments. 
TABLE II. Fluorescence decay parameters of TMA-DPH in various membranes.

\begin{tabular}{|c|c|c|c|c|c|c|c|c|c|c|c|c|}
\hline $\begin{array}{l}\text { membrane } \\
\text { preparation }\end{array}$ & $a_{1}$ & $\stackrel{T_{1}}{(\pi 5)}$ & $\alpha_{2}$ & $\begin{array}{r}r_{2} \\
(\mathrm{~ns})\end{array}$ & $\alpha_{3}$ & $\begin{array}{c}T_{3} \\
\text { (ns) }\end{array}$ & 0.4 & $\begin{array}{c}\tau_{4} \\
(n i s)\end{array}$ & $\begin{array}{l}\langle\tau\rangle \\
\text { (ns) }\end{array}$ & $x^{2}$ & DW & $\mathbf{Z P}$ \\
\hline $\begin{array}{l}\text { platelets } \\
t=0 \mathrm{~min}\end{array}$ & $\begin{array}{l}2038 \\
.034\end{array}$ & $\begin{array}{r}2.39 \\
.08\end{array}$ & $\begin{array}{l}.762 \\
.005\end{array}$ & $\begin{array}{r}7.35 \\
.02\end{array}$ & & & & & 6.89 & 1.15 & 1.88 & 231 \\
\hline $\begin{array}{l}\text { platelets } \\
\mathrm{t}=140 \mathrm{~min}\end{array}$ & $\begin{array}{l}475 \\
.055\end{array}$ & $\begin{array}{r}224 \\
.03\end{array}$ & $\begin{array}{l}.525 \\
.006\end{array}$ & $\begin{array}{r}5.54 \\
.02\end{array}$ & & & & & 4.66 & 1.21 & 1.71 & 158 \\
\hline $\begin{array}{l}\text { tonicated } \\
\text { platellets }\end{array}$ & $\begin{array}{l}388 \\
.0093\end{array}$ & $\begin{array}{r}2.23 \\
.03\end{array}$ & $\begin{array}{l}.612 \\
.004\end{array}$ & $\begin{array}{r}6.14 \\
.01\end{array}$ & & & & & 5.41 & 1.21 & 1.69 & 170 \\
\hline $\begin{array}{l}\text { platellet } \\
\text { dipid }\end{array}$ & $\begin{array}{l}382 \\
.004\end{array}$ & $\begin{array}{r}2.32 \\
.03\end{array}$ & $\begin{array}{l}.618 \\
.005\end{array}$ & $\begin{array}{r}5.69 \\
.011\end{array}$ & & & & & 5.01 & 1.19 & 1.78 & 185 \\
\hline $\mathrm{PC}^{4}$ & $\begin{array}{l}360 \\
.005\end{array}$ & $\begin{array}{r}1.22 \\
.93\end{array}$ & $\begin{array}{l}.640 \\
.006\end{array}$ & $\begin{array}{r}2.95 \\
.01\end{array}$ & & & & & 2.62 & 1.15 & 190 & 211 \\
\hline $\begin{array}{l}\text { PC/cholesterol } \\
C / P=1.4\end{array}$ & $\begin{array}{l}.209 \\
.007\end{array}$ & $\begin{array}{r}3.08 \\
.10\end{array}$ & $\begin{array}{l}.791 \\
.009\end{array}$ & $\begin{array}{r}6.94 \\
.02\end{array}$ & & & & & 6.53 & 0.98 & 1.95 & 234 \\
\hline $\begin{array}{l}\mathrm{PC} / \mathrm{PE} \\
(7 / 3)\end{array}$ & $\begin{array}{l}.439 \\
.007\end{array}$ & $\begin{array}{r}1.08 \\
.08\end{array}$ & $\begin{array}{l}.561 \\
.008\end{array}$ & $\begin{array}{r}2.89 \\
.01\end{array}$ & & & & & 2.48 & 1.15 & 2.15 & 269 \\
\hline $\begin{array}{l}\text { PC/SphM } \\
(7 / 3)\end{array}$ & $\begin{array}{l}.401 \\
.004\end{array}$ & $\begin{array}{r}1.60 \\
.03\end{array}$ & $\begin{array}{l}.599 \\
.006\end{array}$ & $\begin{array}{r}3.72 \\
.01\end{array}$ & & & & & 3.25 & 1.27 & 1.73 & 158 \\
\hline $\begin{array}{l}\text { platelets } \\
1=0 \text { min }\end{array}$ & $\begin{array}{l}.958 \\
.003\end{array}$ & .038 & $\begin{array}{l}.009 \\
.001\end{array}$ & $\begin{array}{r}2.24 \\
.06\end{array}$ & $\begin{array}{l}.032 \\
.001\end{array}$ & $\begin{array}{r}7.31 \\
.02\end{array}$ & & & 6.02 & 237 & 1.29 & 223 \\
\hline $\begin{array}{l}\text { platelets } \\
\text { ti }=140 \text { min }\end{array}$ & $\begin{array}{l}.843 \\
.006\end{array}$ & .038 & $\begin{array}{l}.071 \\
.001\end{array}$ & $\begin{array}{r}1.83 \\
.02\end{array}$ & .086 & $\begin{array}{r}5.38 \\
.01\end{array}$ & & & 4.37 & 1.61 & 1.36 & 28 \\
\hline $\begin{array}{l}\text { platelets } \\
t=0 \mathrm{~min}\end{array}$ & $\begin{array}{l}.964 \\
.004\end{array}$ & .038 & $\begin{array}{l}.004 \\
.001\end{array}$ & $\begin{array}{r}1.35 \\
.21\end{array}$ & $\begin{array}{l}.008 \\
.001\end{array}$ & $\begin{array}{r}4.15 \\
.50\end{array}$ & $\begin{array}{l}.024 \\
.001\end{array}$ & $\begin{array}{r}7.59 \\
.09\end{array}$ & 5.96 & 1.18 & 1.95 & 267 \\
\hline $\begin{array}{l}\text { platelets } \\
t=140 \mathrm{~min}\end{array}$ & $\begin{array}{l}.841 \\
.0110\end{array}$ & .038 & $\begin{array}{l}.036 \\
.001\end{array}$ & $\begin{array}{r}0.81 \\
.04\end{array}$ & $\begin{array}{l}.065 \\
.002\end{array}$ & $\begin{array}{r}3.07 \\
.09\end{array}$ & $\begin{array}{l}.058 \\
.002\end{array}$ & $\begin{array}{r}5.85 \\
.05\end{array}$ & 4.37 & 1.08 & 1.97 & 251 \\
\hline $\begin{array}{l}\text { sonicated } \\
\text { platelels }\end{array}$ & $\begin{array}{l}.817 \\
.006\end{array}$ & .038 & $\begin{array}{l}.031 \\
.002\end{array}$ & $\begin{array}{r}0.99 \\
.07\end{array}$ & $\begin{array}{l}.062 \\
.002\end{array}$ & $\begin{array}{r}3.25 \\
.14\end{array}$ & $\begin{array}{l}.090 \\
.003\end{array}$ & $\begin{array}{r}6.41 \\
.05\end{array}$ & 5.21 & 1.12 & 1.85 & 245 \\
\hline $\begin{array}{l}\text { platelet } \\
\text { liphod }\end{array}$ & $\begin{array}{l}.504 \\
.014\end{array}$ & .038 & $\begin{array}{l}.078 \\
.0074\end{array}$ & $\begin{array}{r}0.81 \\
.05\end{array}$ & $\begin{array}{l}.184 \\
.006\end{array}$ & $\begin{array}{r}3.19 \\
.11\end{array}$ & $\begin{array}{l}.235 \\
.009\end{array}$ & $\begin{array}{r}5.94 \\
.05\end{array}$ & 4.95 & 1.10 & 1.96 & 276 \\
\hline$P C$ & $\begin{array}{l}.784 \\
.009\end{array}$ & .038 & $\begin{array}{l}.065 \\
.001\end{array}$ & $\begin{array}{r}0.65 \\
.01\end{array}$ & $\begin{array}{l}.110 \\
.007\end{array}$ & $\begin{array}{r}2,43 \\
.05\end{array}$ & $\begin{array}{l}.041 \\
.007\end{array}$ & $\begin{array}{r}3.43 \\
.08\end{array}$ & 2,42 & 1.11 & 1.90 & 233 \\
\hline $\begin{array}{l}\text { PC/choldsterol } \\
\mathrm{C} / \mathrm{P}=1.4\end{array}$ & $\begin{array}{l}245 \\
.029\end{array}$ & $.0 \$ 88$ & $\begin{array}{l}.043 \\
.006\end{array}$ & $\begin{array}{r}0.79 \\
.14\end{array}$ & $\begin{array}{l}.178 \\
.016\end{array}$ & $\begin{array}{r}3.74 \\
.26\end{array}$ & $\begin{array}{l}.565 \\
.020\end{array}$ & $\begin{array}{r}7.04 \\
.06\end{array}$ & 6.51 & 1.00 & 1.94 & 243 \\
\hline $\begin{array}{l}\text { PC/PE } \\
(7 / 3)\end{array}$ & $\begin{array}{l}393 \\
.030\end{array}$ & .038 & $\begin{array}{l}.156 \\
.007\end{array}$ & $\begin{array}{r}0.33 \\
.02\end{array}$ & $\begin{array}{l}.193 \\
.005\end{array}$ & $\begin{array}{r}1.27 \\
.05\end{array}$ & $\begin{array}{l}.258 \\
.006\end{array}$ & $\begin{array}{r}2.93 \\
.02\end{array}$ & 239 & 1.14 & 2.15 & 263 \\
\hline $\begin{array}{l}\text { PCSphM } \\
(7 / 3)\end{array}$ & $\begin{array}{l}.832 \\
.005\end{array}$ & .038 & .047 & $\begin{array}{r}0.80 \\
.02\end{array}$ & $\begin{array}{l}.088 \\
.005\end{array}$ & $\begin{array}{r}293 \\
.07\end{array}$ & $\begin{array}{l}.033 \\
.006\end{array}$ & $\begin{array}{r}4.35 \\
.10\end{array}$ & 2.99 & 1.07 & 2,06 & 274 \\
\hline
\end{tabular}

Biexponential analysis (upper part) of fluorescence decay was performed from channel 54 (emission peak at channel 26 , time equivalence per channel was $32.5 \mathrm{ps}$ ). Tri- and tetraexponential analysis (middle and lower part, respectively) started right at the emission peak, with $\tau_{1}$ fixed at $38 \mathrm{ps}$. Fit quality can be judged from $\chi^{2}$, the Durbin-Watson parameter (DW), and the mumber of zero passages in the autocorrelation function (ZP). Standlard deviations of fitted parameters are indicated underneath. See also Fig.5. for graphical representation of the most relevant calculated parameters. All measurements were carried out at $37^{\circ} \mathrm{C}$ as described in the methods. Data shown are representative for 3 or more independent determinations. 
dynamic properties are influenced by probeprobe interactions, or that the structural properties of the membrane are dusturbed by the presence of the probe in the bilayer. To ensure that the observed anisotropies are not influenced by TMA-DPH itself, the dependence of the anisotropy on the concentration of TMADPH was determined. It was found that increasing the TMA-DPH concentration to $2 \mu \mathrm{M}$ did not affect the observed anisotropies $(0.260$ -0.261 ), while at $5 \mu \mathrm{M}$ TMA-DPH the anisotropy was significantly reduced $(0.260-0.253)$. Therefore, it is concluded that under the conditions employed the observed anisotropies of TMA-DPH are representative for the structural and dynamic state of its lipid environment.

Of major interest was the question what kind of lipid environments TMA-DPH would experience during its movement from the outer monolayer of the platelet plasma membrane to the intracellular membranes. For this purpose the fluorescence lifetime and the steady-state anisotropy of TMA-DPH were determined as a function of the bound fraction of TMA-DPH, which proved a better representation tationtationtationtationtationtation of the level of penetration than the incubation time with TMADPH. It was found that, during progressive penetration of TMA-DPH into unstimulated platelets, the average fluorescence lifetime $(\langle\tau\rangle)$ and the steady-state anisotropy $\left(r_{s}\right)$ decreased dramatically (Fig. 4). $\langle\tau\rangle$ decreased from 6.9 to $4.5 \mathrm{~ns}$, while $\mathrm{r}_{\mathrm{s}}$ dropped from 0.260 to 0.210 . Both parameters seem to level off at about 5 to 6 times the original bound fraction of TMADPH, and follow a similar time course as the penetration of TMA-DPH (Figs. 1 and 3). Thus, intracellular lipid compartments are much more fluid than the outer monolayer of the plasma membrane.

To discriminate between static and dynamic components of lipid fluidity, the structural order parameter (S) and the rotational diffusion constant $\left(D_{1}\right)$ are also presented as a function of the bound fraction of TMA-DPH (Fig. 4). S decreased dramatically during penetration of TMA-DPH, in a manner identical to that of $\langle\tau\rangle$, from 0.88 to 0.77 . Such a close rellationship between $S$ and $\langle\tau\rangle$ was also observed by Straume and Litman $[617,618]$. They suggested that a decrease in order will be accompanied by an increased penetration of water into the lipid bilayer. The resulting increase in polarity will reduce the fluorescence lifetime of TMA-DPH. Examination of all the data, irrespective of the type of membrane, confirmed this close relationship between $S$ and $\langle\tau\rangle$. The behavior of $D_{\perp}$ during penetration of TMA-DPH into platelets clearly deviates from the behavior of $S$ : a fast increase is observed, from 10 to about $80 \mathrm{MHz}$, followed by a relatively stable plateau, which is already reached when the bound fraction of TMA-DPH is doubled. As such, the observation that an increase in rate of rotational motion $\left(D_{\perp}\right)$ accompanies an decrease in order (S) is in line with observations employing DPH [320] of TMA-DPH [620]. However, the observation that $D_{\perp}$ reaches a plateau while $S$ further decreases may reveal some interesting details about the environment sensed by TMA-DPH in the platelet interior (vide infra).

\section{Characterization of membrane components determining the behavior of TMA-DPH in membranes.}

First of all, some evidence will be provided for the location of TMA-DPH in the lipid bilayer, since this is crucial to develop a detailed interpretation of changes in fluorescence lifetime and anisotropy. TMA-DPH was originally synthesized to obtain a membrane probe with a more defined distribution in the bilayer than DPH [519]. Subsequently, this assumption was confirmed by severa] authors showing that TMA-DPH was unimodally distributed in lipid bilayers [182, 617]. However, a more precise description of the location of TMA-DPH in the lipid bilayers has not been obtained, though it can be expected that TMA-DPH will locate its positive charge at the aqueous boundary of a membrane. Quenching experiments indeed showed that TMA-DPH must be located close to the surface of the membrane [137]. Based on the succesful application of TNP-Gly to quench DPH [577], and taking advantage of the positive charge of TMA-DPH, the location of TMADPH in the lipid bilayer was investigated with the aid of TNP-Lys and TNP-Glu, which are neutrally and negatively charged, respectively. The differential effects of both quenchers on the average fluorescence lifetime of TMA-DPH, should allow conclusions about its exact location. Fluorescence lifetimes of TMA-DPH in 
zwitterionic $\mathrm{PC}$ vesicles and negatively charged PCAPS vesicles, in the presence and absence of TNP-Lys or TNP-Glu, were determined by phase fluorimetry. It was found that TNP-Lys was more effective than TNP-Gilu in quenching TMA-DPH in PC vesicles (Table III). This is a surprising result, since TNP-Gilu, being negatively charged, was expected to quench the positively charged TMA-DPH better than TNPLys. These results can only be explained, taking into account the zwitterionic character of $\mathrm{PC}$. TNP-Glu would not reach the phosphate layer but rather stay close to the positively charged choline headgroup, while TNP-Lys would be located close to the negatively charged phosphate group of PC. In line with this reasoning, it is concluded that the ternary amino-group of TMA DPH is in close proximity of the negatively charged phosphate-group of $\mathrm{PC}$. This conclusion is further confirmed by the effect of addition of PS to PC vesicles (Table III). Applying a negative surface charge, it can be expected that the binding of TNP-Lys to the membrane surface is greatly enhanced. This does not, however, result in more quenching of TMA-DPH. On the contrary, quenching decreases. Again, this result can be explained by a location of TMA-DPH away from the headgroup, anchored with its charge in the phosphate region.

The motional characteristics of TMA-DPH in a biological membrane can be expected to reflect both bulk lipid properties and the presence of proteins [320]. To evaluate both factors, the fluorescence properties of TMADPH in sonicated platelets and vesicles from total platelet lipids were investigated (Fig. 5 , tables I and II). In platelet lipid vesicles $\langle\tau\rangle$ and $r_{s}$ of TMA-DPH were $5.00 \pm 0.06 \mathrm{~ns}$ and $0.226 \pm 0.003$ (mean $\pm s . d, n=7$ ). These values are much lower than those from the outer monollayer of the platelet plasma membrame, again indicative for a fluidity gradient ower the plasma membrane. In sonicated plitelets $\langle t\rangle$ and $\pi_{s}$ of TMA-DPH amounted to $5.39 \pm 0.03 \mathrm{~ns}$ and $0.241 \pm 0.002$ (mean \pm s.d., $\mathrm{n}=3$ ). The higher order in sonicated platelets with respect to platelet lipid vesicles can be explained by the presence of membrane proteins, which are known to increase the lipid order in bilayers $[105,321,620]$.

To investigate the relative importance of individual membrane lipid components in determining lipid order as sensed by TMA-DPH, a systematic survey of the major lipid species of the platelet plasma membrane was performed. From the data presented in Fig. 5 and Tables 1

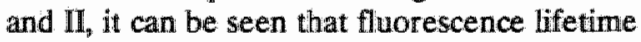
and anisotropy of TMA-DPH in PC vesicles have much smaller values than those in all platelet systems. Inclusion of 30 mole\% PE, PS or SphM shows that these lipids are not major determinants of fluorescence lifetime and anisotropy of TMA-DPH in lipid bilayers, and that SphM is more effective than PE or PS. Inclusion of 58 mole\% cholesteroll (C/P ratio 1.4) clearly shows that cholesterol is by far the major determinant of fluorescence lifetime and anisotropy of TMA-DPH in lipid bilayers, even when the higher molar levell of cholesterol ( $58 \%$ ) relative to $\mathrm{PE}, \mathrm{SphM}$, and $\mathrm{PS}(30 \%)$ is taken into account. The importance of cholesterol for the flworescence properties of TMADPH is also demonstrated by platelet lipid vesicles, which contained 35 mole\% cholesterol. A similar important role of cholesterol for the fluorescence properties of TMA-DPH in lipid bilayers is reported by Straume \& Litman [618] and by Deinum et.al . [155]. It should be mentioned that the unsaturation of phospholipid acyl chains also affects lipid order as sensed by TMA-DPH $[155,617]$ : more unsaturation results in decreased lipid order. Quantitativelly, the effect of acyl chains on fluorescence lifetime and lipid order is relatively small, but may be responsible for differences observed between PC, PS, and PE.

Special attention is required by the $d y-$ namic component of fluidity expressed as the rotational diffusion coefficient $\left(D_{\perp}\right)$. This parameter changed during penetration of TMA-

Table IV. Differential influence of major membrane components on fuorescence lifetime and anisotropy of TMA-DPH.

$\begin{array}{lcccc}\text { membrane component } & <\tau> & \text { is } & S & \text { D } 1 \\ \text { protein } & - & + & + & - \\ \text { sphingomyelin } & + & - & + & -1+ \\ \text { acy-chain unsatiration } & - & - & - & + \\ \text { chiolesterol } & + & + & + & +\end{array}$

This table is a qualitative representation of Fig. 5 , designating the relative contribution of major membrane components to fluorescence lifetime and motional properties of TMA-DPH. The relative contribution can either be of minor importance $(-)$, of significant importance $(+)$, or of major importance $(++)$ to the parameter in question. 
DPH into unstimulated platelets in a way different from the other parameters (Fig. 4). $D_{\perp}$ very rapidly increases to a high plateau-like level, while the other parameters change smoothly during penetration of TMA-DPH into platelets. In addition, even the highest rate of motion, observed with egg-PC alone, is still lower than $\mathbf{D}_{\perp}$ in platelets after more than one bour labeling with TMA-DPH ( 60 vs $80 \mathrm{MHz}$ ). Apparently, $D_{\perp}$ senses a specific aspect of intracellular platelet membranes which is not revealed by the other parameters. Neither SphM nor cholesterol can be responsible for these fast movements, considering their enrichment in the outer monolayer. Intracellular proteins also have to be excluded, since proteins decrease motional rates due to their own much slower motion. Lowering the extent of acyl chan unsaturation is accompanied by slower motion of TMA-DPH [617]. Thus, the high $D_{\perp}$ values observed when TMA-DPH enters the platelet interior, can be explained by the specific enrichment of arachidonic acid in the inner monolayer of the platelet plasma membrane [494].

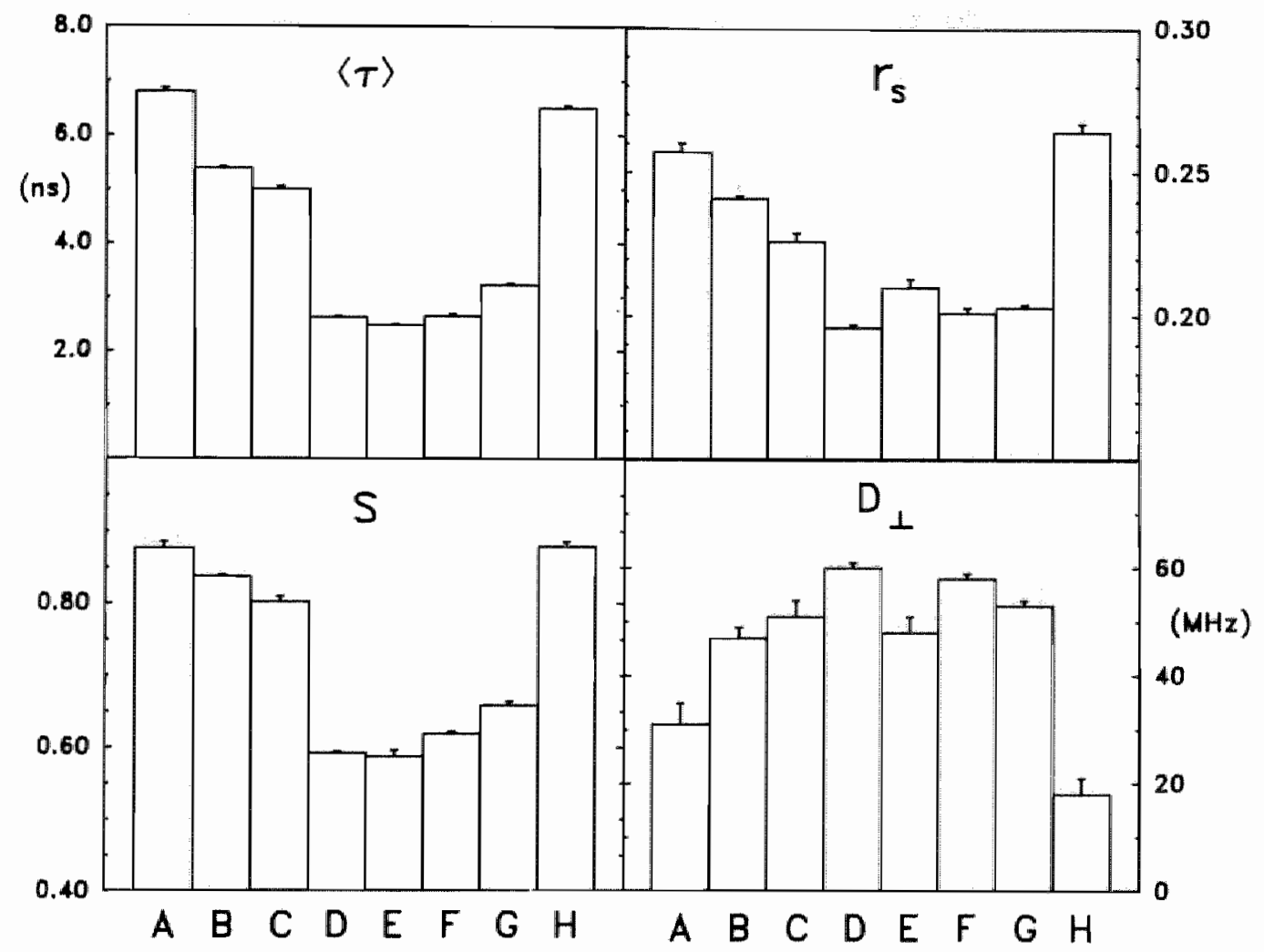

Fig. 5. Fluorescence lifetime and motional properties of TMA-DPH in various membrane systems. Presented are the average fluorescence lifetime, $\langle\tau\rangle$, the steady-state anisotropy, $\mathrm{r}_{\mathrm{s}}$, the structural order

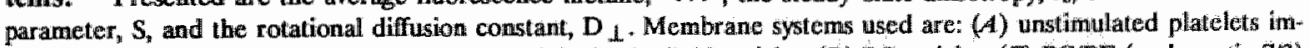
mediately after labeling, (B) sonicated platelets, $(C)$ platelet lipid vesicles, (D) PC vesicles, (E) PC/PE (molar ratio 7/3) vesides, $(F)$ PC/PS $(7 / 3)$ wesicles, $(G)$ PC/SphM $(7 / 3)$ vesicles, $(H)$ PC/cholesterol vesicles, C/P ratio 1.4. Platelet preparations were equivalent to $2: 10^{7}$ platelets per ml. Vesicle preparations contained 25 to $50 \mu \mathrm{M}$ phospholipid per ml. Experimental details are described in the methods. Data shown are the mean 1 s.d. of at least 3 separate measurements. 
Further characterization of the effect of cholesterol on the behavior of TMA-DPH in membranes

Since it was found that cholesterol is the major determinant of both fluorescence lifetime and anisotropy of TMA-DPH in membranes, the effects of cholesterol were considered in more detail by investigation of its dose-response relationship. It was found that by increasing the cholesterol content in $\mathrm{PC}$ vesicles from zero to a $\mathrm{C} / \mathrm{P}$ ratio of 1.8 , $\langle\tau\rangle$ increased from $2.63 \pm 0.03$ to $7.17 \pm 0.04 \mathrm{~ns}$ (mean \pm s.d., $n=3$ ), while $S$ increased from $0.600 \pm 0.004$ to $0.900 \pm 0.003$ (mean \pm s.d., $n=3$ ). Unilike $\langle\tau\rangle$, which increases allmost linearly with cholesterol content, $\mathrm{S}$ clearly levels off at $\mathrm{C} / \mathrm{P}$ ratios higher than 1 . The relation between cholesterol content and $S$ is in line with the obserwation that the condensing effect of cholesterol [159], and its effect on microviscosity [131] and steady-state fluorescence polarization [62] level off at high $C / P$ ratios. The dependence of $r_{s}$ on cholesterol content in $P C$ vesicles obviously combines the behavior of $\mathrm{S}$ and $D_{1,}$ as could be expected from its nature.

The relationship between cholesterol content and the rotational dynamics of TMA-DPH was found to be biphasic. $\mathrm{D}_{\perp}$ increases at low cholesterol concentrations from about 60 to 70 $\mathrm{MHz}$, followed by a decrease down to about 10 $\mathrm{MHz}$ at a C/P ratio of 1.8 . This biphasic behavior has not been observed before, and may be related to the specific location of TMA-DPH in the lipid bilayer. In general an inverse relation. ship between $S$ and $D_{\perp}$ is observed, explaining the decreasing part of the $D_{\perp}$ curve. The increase in $D_{\perp}$ of TMA-DPH with cholesterol at lower $\mathrm{C} / \mathrm{P}$ ratios is in agreement with findings from Straume \& Litman [618], who used various $\mathrm{PC}$ vesicles with $\mathrm{C} / \mathrm{P}$ ratios up to 0.4 . The peak of $D_{\perp}$ around 20 mole\% cholesterol may be explained by its concentration-dependent organization in lipid bilayers of $\mathrm{PC}$, which changes significantly around 20 mole $\%$ [for review see: 325,579 ]: below about 20 mole\% cholesterol occurs in cholesterol-rich domains, while above 20 mole $\%$ cholesterol is homogenously distributed, and at even higher choleste-

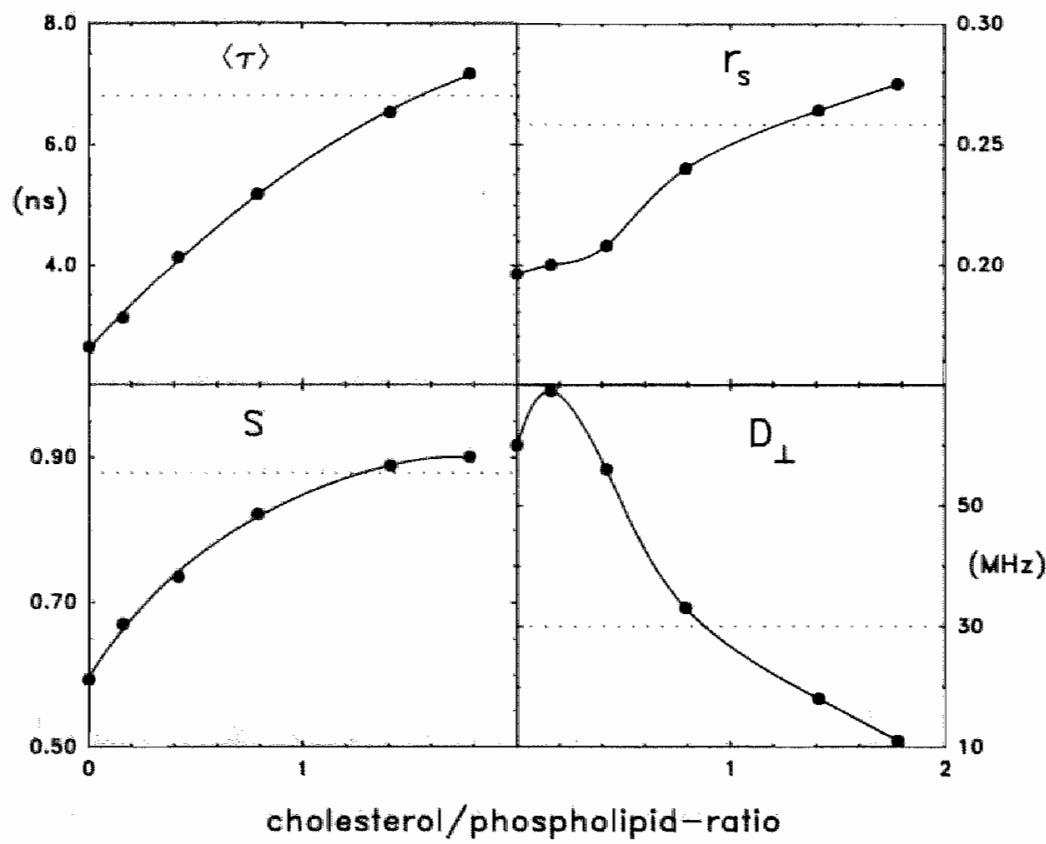

Fig. 6. Fluorescence lifetime and motional properties of TMA-DPH in PC vesicles with various amounts of cholesterol. Presented are the average fluorescence lifetime, $\langle\tau\rangle$, the steady-state anisotropy, $\mathbf{r s}_{\mathrm{s}}$ the siructural order parameter, $S$, and the rotational diffusion constant, $D_{\perp}$. Data shown are the mean of two separate measurements. Standard deviations fall within symbols. Dotted lines represent the average walues of TMA-DPH in unstimulated platelets inmediately after labeling. Experimental details are described in the methods. 
rol levels pure cholesterol domains may separate out.

\section{DISCUSSION}

Application of TMA-DPH as a reporter of structural membrane properties, and time-resolved fluorescence spectroscopy to monitor and analyze both fluorescence lifetime and static as well as dynamic components of fluidity, appeared to present a versatile combination allowing extrapolation of fundamental properties of membrane fluidity to biological membranes, and revealing detailed information about the organization of platelet membranes. Following the fate of TMA-DPH after addition to unstimulated platelets, a gradual penetration into the platelet interior could be demonstrated both by fluorescence intensity measurements, and by making use of the sensitivity of time-resolved fluorescence. Hyperbolic fitting of both the increase in non-dilutable F (Fig. IB) and the increase in membrane-bound fraction of TMA-DPH (Fig. 3), shows that after approximately 6 hours all platelet membranes will be labeled. Halfmaximal penetration of TMA-DPH into platelets is obtained after 2 hours.

The gradual penetration of TMA-DPH is accompanied by progressive changes in both fluorescence lifetime and anisotropy of TMA$\mathrm{DPH}$, indicating that the intracellular platelet membranes are much more fluid than the outer monolayer of the platelet plasma membrane. In general, the observed difference in fluidity can be due to quantitative changes in one or more of the following membrane components: (1) cholesterol $[62,155,313,320,618]$, (2) sphingomyelin $[25,62],(3)$ acyl chain unsaturation $[62$, $155,320,617,621]$, and (4) membrane proteins $[105,320,321]$.

Cholesterol is not evenly distributed over the various platelet membranes. Membranes from the dense tubular system contain less cholesterol than the plasma membrane [186, 195, $243,358,418$ ]: C/P ratio about 0.4 vs. 0.7 , respectively. Secretory granules contain even less cholesterol $[243,556]$ : C/P ratio between 0.20 and 0.25 . In view of the dramatic effects of cholesterol on membrane fluidity (Figs. 5 and 6), it is clear that a decrease in cholesterol content in intracellular membranes will be responsible for a significant part of the observed fluidity gradient.

Sphingamyelin is also not homogenously distributed throughout the platelet. The platelet plasma membrane is enriched in sphingomyelin $[195,358,556]$. Again, such a distribution of sphingomyelin would favor the observed increase in fluidity of intracellular membranes, in view of its effects on membrane fluidity (Fig. 5). The contribution of sphingomyelin to the fluidity gradient is enhanced by its extreme asymmetric distribution over the plasma membrane. More than $90 \%$ of sphingomyelin in the platelet plasma membrane is located in the outer monolayer $[98,494]$.

Acyl chain unsaturation will further augment the fluidity gradient. About $60 \%$ of all esterified arachidonic acid in platelets is present in the plasma membrane, the majority of which residing in the cytosolic leaflet [494]. The contribution of acyl chain unsaturation to the $o b$ served fluidity gradient in platelets is further demonstrated by the observation that polyunsaturated fatty acids provide about $30 \%$ of the acyl chains in the $s n-2$ position of phospholipids in the outer monolayer of the platelet plasma membrane [494], while total platelet phospholipids contain about $50 \%$ polyunsaturated fatty acids in the $s n-2$ position [396]. Direct evidence for the involvement of acyl chain unsaturation in the fluidity gradient can be obtained from the changes in rotational diffusion constant $\left(D_{\perp}\right)$ during gradual penetration of TMA-DPH into platelets. During penetration of TMA-DPH into unstimulated platelets (Fig. 4), $D_{\perp}$ increases to levels higher than obtained with cholesterol-free PC vesicles (Fig. 5). It was argued, in agreement with other observa* tions [617], that an increase in acyl chain unsaturation can cause these high rotational rates. In this way, earlier observations about transbilayer asymmetry of acyl chain unsaturation [494] are confirmed. Another possibility which should be considered, is the interaction between cytosolic proteins with intracellular membranes (vide infra).

Proteins decrease membrane fluidity, due to their relative slow motional characteristics in comparison with lipids [105, 321]. This is evident from the difference between sonicated platelets and vesicles from platelet lipids (Table II). Thus, proteins would counteract the observed fluidity gradient in platelets. Besides membrane proteins, cytosolic proteins could, in 
principle, also infuence the fluorescence properties of TMA-DPH. This is demonstrated by the binding of TMA-DPH to albumin [166], which is characterized by a very high anisotropy. A contribution of binding of TMA-DPH to cytosolic proteins, however, seems unlikely for the following reasons. During extensive penetration of TMA-DPH into platelets, the steadystate anisotropy approaches values obtained only in membranes free of cholesterol and proteins (Fig. 5), excluding an involvement of cytosolic proteins in the fluorescence properties of TMA-DPH, since proteins significantly increase lipid order [105, 321]. In addition, the increase in binding of TMA-DPH to platelets during its penetration is in reasonable agreement with the increase which would be expected from the ratio of phospholipids between outer monolayer and total platelets.

From the above discussion it is obvious that the observed increase in membrane fluidity during penetration of TMA-DPH into unstimulated platelets is in line with current findings about the compositional distribution of lipids throughout the platelet. Generally speaking, the platelet intracellular membranes must have a fluidity approaching that of egg-PC vesicles, while the outer monolayer displays a membrane fluidity only found with vesicles containing more than 50 mole\% cholesterol (compare Figs. 4 and 5). Detailed conclusions about the relative contributions of the various intracellular membranes to the observed fluidity gradient can not be extracted from the averaged fluorescence lifetime and motional properties of TMADPH. Moreover, considering the rapid equilibrium between membrane and aqueous compartments of TMA-DPH, it is anticipated that intracellular TMA-DPH will diffuse rapidly throughout the platelet, providing only aspecific information about the fluidity of intracellular platelet membrane compartments.

Owing to the specific initial incorporation of TMA-DPH in the outer monolayer of the platelet plasma membrane, however, more detailed information about the membrane fluidity of the outer leaflet of the plasma membrane can be obtained. From the data presented in Fig. 5, several conclusions can be inferred designating the relative importance of the various membrane components in determining the fluorescence properties of TMA-DPH in membranes (Table IV). Comparison of sonicated platelets and platelet lipid vesicles shows that the presence of membrane proteins is especially reflected by $r_{\mathrm{s}}$ and $S$, while $\langle\tau\rangle$ and $D_{1}$ are less influenced by proteins. Judged from $S$ and $r_{S}$ it appears that proteins do not contribute much to the membrane fluidity of the outer monolayer. Of the lipid components, only cholesterol has major effects on membrane fluidity, as judged by all parameters. Sphingomyelin is less effective, especially when $r_{s}$ is ewaluated. Acyl chain unsaturation is also of minor importance relative to cholesterol, except for its effect on $D_{1}$. Thus it can be concluded that cholesterol is the most important determinant of the fluidity of the outer monolayer of the platelet plasma membrane, suggesting that the fluidity gradient over the plasma membrane is mainly due to an asymmetric transbilayer distribution of cholesterol.

Quantitative conclusions about the cholesterol content of the outer leaflet of the platelet plasma membrane may be drawn from the cholesterol dose-response relationships. The fluorescence lifetime of TMA-DPH seems most appropiate to relate to cholesterol content, since it is hardly sensitive to proteins and acyl-

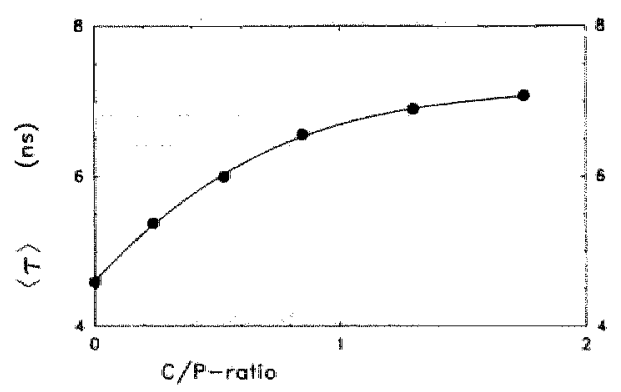

Fig. 7 Estimation of the C/P ratio in the outer monolayer of the platelet plasma membrane from the fluorescence lifetime of TMA-DPH in cholesterol containing vesicles. The solid line represents the cholesterol-dependence of the average fluorescence lifetime of TMA-DPH in vesicles containing the following mixture of phospholipids: SphM/PCIPE $(6 / 3 / 1$, molar ratio). All phospholipids were from egg-yolk (Sigma, St.Louis MO, USA). The upper dotted line represents the outer monolayer of the platelet plasma membrane as sensed by TMA-DPH. The lower dotted line is derived from the upper one by substraction of the difference between sonicated platelets and platelet lipid, taken to represent the maximal contribution of proteins. Data were taken from a representative experiment. Experimental details are described in the methods. 
chain unsaturation (Table IV). Egg-PC vesicles, however, do not qualify as representative for the outer monolayer of the platelet plasma membrane. Therefore, the cholesterol dependence of the lifetime of TMA-DPH was determined in vesicles containing SphM, $P C$ and $P E$ in a molar ratio of 6,3 and 1 , respectively, resembling the outer leaflet of the plasma membrane $[98,494]$. It can be seen in Fig. 7 that the lifetime of TMA-DPH in the outer leaflet would reflect a C.P ratio of 1.15 , if the effect of proteins is not taken into account. If the difference between sonicated platelets and platelet lipid vesicles is taken to represent the effect of proteins on the fluorescence lifetime of TMA-DPH, then a C/P ratio of 0.75 is estimated for the outer leaflet. It can be argued, however, that the correction for the effect of proteins is an overestimation. Most of the difference in fluorescence lifetime of TMA-DPH between sonicated platelets and platelet lipid vesicles is due to intracellular proteins, as is suggested by the effect of $\mathrm{Ca}^{2+}$-dependent proteolysis of cytosolic proteins prior to sonication (see next chapter). Thus, the best estimate for the cholesterol content of the outer monolayer of the platelet plasma membrane would be a $C / P$ ratio of about 1 .

An independent estimation of the cholesterol content of the outer monolayer of the platelet plasma membrane can be obtained from the work of Shattil and Cooper [593]. These authors manipulated the cholesterol content of platelets by incubation with cholesterol-rich vesicles. They found that only vesicles with a $C / P$ ratio higher than 1.0 were able to enrich platelets in cholesterol. Assuming a cholesterol exchange process between the outer monolayer of vesicles and platelets $[567,715]$, this finding suggests a C/P ratio of 1.0 for the outer leaflet of the platelet plasma membrane.

Another independent estimation of the cholesterol content in the platelet outer monolayer can be derived from the work of Donner and Stoltz [166], who used TMA-DPH to investigate platelets and erythrocytes. These authors found that TMA-DPH displays the same steadystate anisotropy in platelets and erythrocytes, immediately after labeling. Considering that the phospholipid composition of the outer monolayer of erythrocytes and platelets are similar [731], and that cholesterol is the major determinant of membrane fluidity in the erythrocyte [105], the similarity in anisotropy of
TMA-DPH is taken to indicate that the outer monolayer of both erythrocyte and platelets contain similar amounts of cholesterol. The C/P ratio of erythrocyte membranes is 0.85 to 0.90 $[102,105,365]$. In addition, cholesterol is asymmetrically distributed over the plasma membrane: approximately two-third is located in the outer monolayer $[201,261]$, equivalent to a C/P ratio of 1.1 to 1.2. Thus, comparison of platelets and erythrocytes suggests a CP ratio for the platelet outer monolayer of at least 1.0 .

Having estimated the cholesterol content of the outer leaflet of the platelet plasma membrane, the cholesterol content of the inner leaflet can be calculated from the $C / P$ ratio of the whole plasma membrane. Values for the $\mathrm{C} / \mathrm{P}$ ratio of the whole platelet plasma membrane vary from $0.6[186]$ to $0.66[243]$ and $0.74[358$, 418]. Taking a CPP ratio of 1.0 for the outer monolayer, as argued above, and a C/P ratio of 0.7 for the whole plasma membrane, it can be calculated that the C/P ratio of the inner monolayer would be about 0.4 . This would constitute a considerable transbilayer asymmetry of cholesterol. It should be mentioned here that the suggested asymmetrical distribution of cholesterol is indirectly measured, being derived from the motional properties of TMA-DPH. Clearly, a direct determination, making use for instance of the fluorescent sterol dehydroergosterol [579], would be desirable, and should be employed to confirm the observed cholesterol asymmetry.

Transbilayer asymmetry of membrane fluidity has been observed in erythrocytes [163, $428,587,634,703$ ], in sarcoplasmic reticulum [429], and in fibroblasts $[237,318,578,707]$. In comparison to the erythrocyte, the fluidity gradient over the platelet plasma membrane seems to be more extreme. This is suggested from the results of Donner and Stoltz [166], who observed that the time-dependent decrease in $r_{s}$ of TMA-DPH, indicative for a fluidity gradient, was greater in platelets than in erythrocytes. An asymmetric transbilayer distribution of cholesterol is found to be associated with the observed transmembrane fluidity gradient in erythrocytes $[184,201,261]$, and fibroblasts [261, 318]. In addition, transbilayer asymmetry of cholesterol is reported for Mycoplasma cells [59], and myelin [92]. Thus, the existence of a transmembrane fluidity gradient, coupled to an asymmetric transbilayer distribution of cholesterol, is not a rare phenomenon 
and may turn out to be a general feature of the lipid organization in bilological membranes.

The existence of a transmembrane cholesterol gradient raises several questions concerning its regulation and maintenance. The rate of transbilayer movement (flip rate) of cholesterol is very rapid, with a half-time of seconds $[579,715]$. These high flip rates are not necessarily at odds with a stable transmembrane graldient of cholesterol, since additional factors may contribute to the maintenance of the gradient. Such factors could determine the equilibrium distribution of cholesterol, independent of the dynamics of cholesterol motion, by increasing the affinity for cholesterol of one monolayer. In view of the preferential affinity of cholesterol for sphingomyelin [158], the extreme asymmetric transbilayer distribution of sphingomyelin in the platelet plasma membrane $[98,494]$ could explain an enrichment of cholesterol in the outer monolayer. Membrane proteins provide another candidate for binding cholesterol. Significant binding of cholesterol to membrane proteins has been demonstrated in erythrocytes [586,716], in brush border plasma membranes [63], and in model systems [17, 598]. Whether membrane proteins can bind cholesterol preferentially in one monolayer of a membrane is not known, leaving their role in maintenance of cholesterol transbilayer asymmetry uncertain.

The occurrence of a fluidity gradient over the platelet plasma membrane may contribute significantly to the functional asymmetry of the plasma membrane, which is outlined in the introduction. At the cytosolic side of the plasma membrane, the high fluidity will favor proteinlipid interactions [184], important for the action of phospholipases [157] and the binding of peripheral proteins, like cytoskelletal proteins $[256,458]$. Interaction of cytoskeletal proteins with the cytosolic leaflet of the plasma membrane is likely to involve binding of amphipathic helices to the lipid bilayer surface $\llbracket 176$,
665]. It is conceivabile that shallow pemetration of amphipatic helices into the lipid bilayer leads to spacing of headgroups resulting in a lower lipid order within the lipid bilayer. Decreased lipid order is accompanied by increased rates of motion $[320,620]$. It is emphasized that $D_{\perp}$ increases rapidly during penetration of TMADPH into platelets to levels which are much higher than those found for cholesterol-free membranes. Thus, it is suggested that interaction of gytoskeletal proteins with intracellular membranes is a common phenomenon in platelets. Such interactions between cytoskeletal proteins and the lipids of the inner leaflet of the plasma membrane have been implicated in the maintenance of phospholipid transbilayer asymmetry [54, 733]. Thus, the high fluidity of the inner leaflet of the plasma membrane may be linked to the regulation of transbilayer lipid asymmetry.

The high viscosity of the outer monolayer clearly suits its function as a permeability barrier, since high levels of cholesterol result in low permeability of the lipid bilayer towards non-elektrolytes [159]. In addition, incorporation of cholesterol decreases the flip rate of phospholipids [40, 424]. Thus, the high viscosity of the outer leaflet of the plasma membrane may contribute to the maintenance of phospholipid transbilayer asymmetry.

\section{ACKNOWLEDGEMENTS}

Dr. Gert H.M. Kronenberg (Department of Plant Physiological Research, Agricultural University, Wageningen, The Netherlands) is gratefully acknowledged for making phase fluorimetry available to us, and Dr. Arie van Hoek (Department of Mollecular Physics, Agricultural University, Wageningen, The Netherlands) for assistence with laser fluorimeter. 


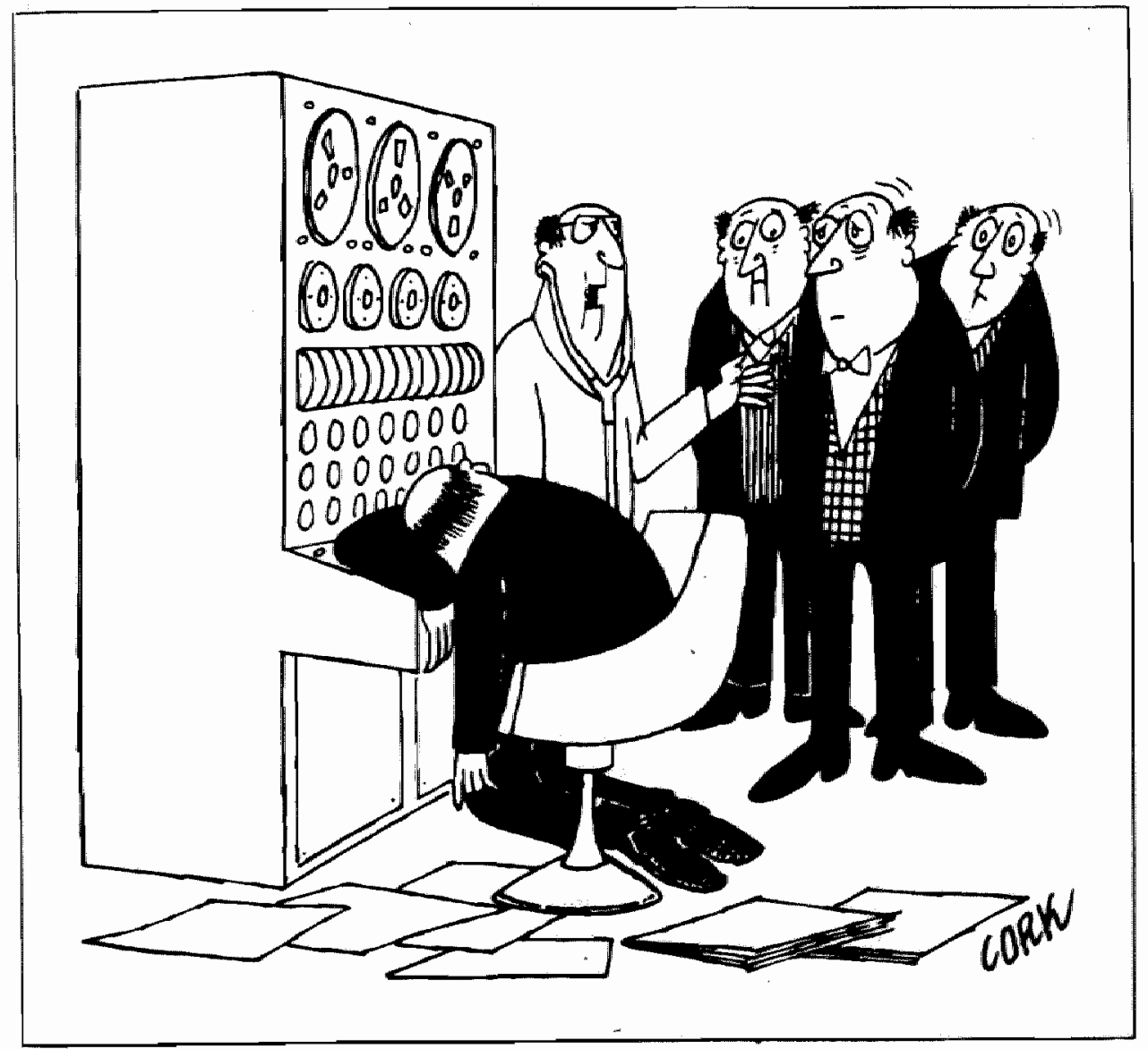




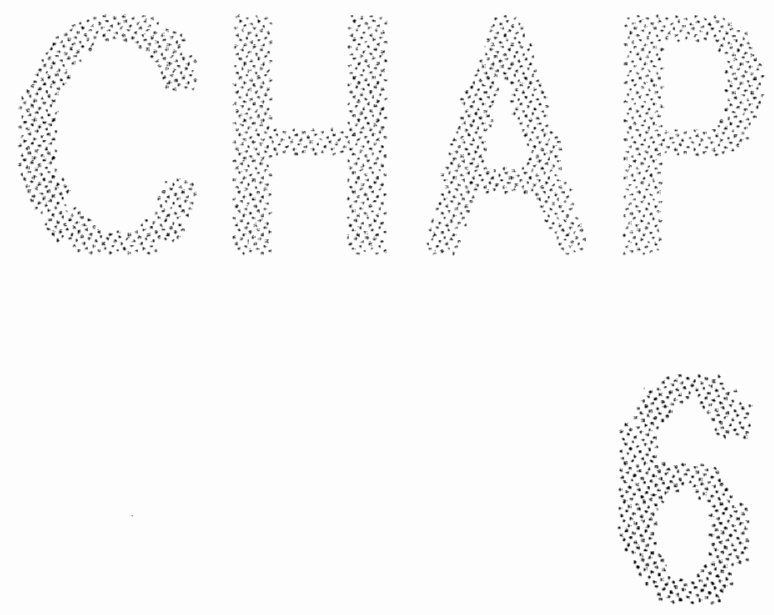




\title{
Rapid transbilayer movement in the platelet plasma membrane during cytoskeletal degradation by calpain
}

\begin{abstract}
The structural integrity of the human platelet plasma membrane during stimulation of rapid transbilayer movement of phospholipids was investigated employing the fluorescent membrame probe TMA-DPH (trimethylammonium-diphenylhexatriene) as a reporter of both membrane structural properties and rates of aspecific transbilayer movement. Fluorescence intensity as well as time-resolved fluorescence were applied to describe the fuorescence properties and location of TMA-DPH. In unstimulated platelets transbilayer movement was relatively slow, with a halftime of 60 to 90 min. Stimulation of platelets by thrombin resulted in an increase of membrane-bound TMA-DPH, explained by the release reaction, and an increase of lipid order in the outer monolayer of the plasma membrane, but did not change transbilayer movement of TMA-DPH, and did not affect the presence of the fluidity gradient over the plasma membrane. Stimulation of platelets by ionomycin gave rise to rapid and massive transbillayer movement of TMA-DPH (halfime 10 to $20 \mathrm{sec}$ ), accompanied by a loss of the transmembrane nuidity gradient and an increased fluidity of the outer monolayer of the plasma membrane. The high rate of transbilayer movement during stimulation by ionomycin suggests the presence of local defects in lipid bilayer structure (nipsites). Further examination of nipsites during platelet stimulation by ionomycin showed that these sites were only temporarily present, and were $\mathrm{Ca}^{2+}$-dependent with a halfmaximall activity around $20 \mu \mathrm{M}$ free $\mathrm{Ca}^{2+}$. Agonist-, time-, and $\mathrm{Ca}^{2+}$-dependence of flipsites are similar to those of cytoskeletal degradation by calpain [Verhallen et.al. (1987), Biochim.Biophys.Acta, 903: 206], suggesting that cytoskeletal reorganization is involved in the generation of nipsites. This suggestion is further supported by the $\mathrm{Ca}^{2+}$-dependent generation of nipsites in nuoride-treated platelets.
\end{abstract}




\section{INTRODUCTION}

The plasma membrane of the blood platelet is an interesting object to study the regulation of transbilayer lipid asymmetry. The lipids are unequally distributed among the two leaflets of the plasma membrane: phosphatidylethanolamine (PE) and phosphatidylserine (PS) are respectively enriched and almost totally located in the cytosolic leaflet, while the outer monolayer of the platelet plasma membrane is enriched in phosphatidylcholine (PC) and cholesterol, and contains almost all sphingomyelin (SphM) $[98,494,569$, previous chapter]. The phospholipid transbilayer asymmetry resembles that of the erythrocyte $[666,736]$. In contrast to the erythrocyte, however, transbilayer asymmetry can be dramatically changed upon cell activation [53]. Depending on the stimulus, phospholipid transbilayer asymmetry of the plasma membrane is more or less lost during platelet activation [50]. As a result of this randomization of phospholipids, PS becomes exposed at the outer surface of the plasma membrane, where it accelerates blood clotting [543a]. The activation-dependent loss of phospholipid transbilayer asymmetry is a rapid phenomenon: when platelets are activated by a calcium ionophore, phospholipid asymmetry is completely lost within one minute [664].

In general, two different mechanisms can account for alterations in transbilayer lipid asymmetry: translocation mediated by an ATPdependent transport protein (translocase) [128, $144,406,603,643,721,722$ ], and transbilayer movement (flipflop) along local disturbances of the lipid bilayer (flipsites) [39, 154].

The existence of a translocase in platelets has been demonstrated [623]. Although the translocase is normally observed by the unidirectional transport of aminophospholipids towards the cell interior, the possibility of outward transport has been suggested recently [702]. However, involvement of a translocase in the activation-dependent loss of transbilayer asymmetry of the platelet plasma membrane seems, a priori, unlikely for the following reasons: (1) the translocase specifically transports amino-phospholipids, while rapid flipflop in activated platelets also concerns SphM and PC [50]; (2) stimulation of rapid flipflop in platelets can be observed only at relatively high cytosolic
$\mathrm{Ca}^{2+}$ levels (higher than $1 \mu \mathrm{M}$ ) $[664]$, at which translocase activity is inhibited in erythrocytes [721]; (3) the inward fliprate in activated platelets ( ${ }^{1 / 2}$ less than $1 \mathrm{~min}$, [664] is more rapid than outward transport rates of the translocase ( $\mathrm{t}_{1 / 2}$ for $\mathrm{PS}$ about $7 \mathrm{~min}$ at $37^{\circ} \mathrm{c}$, [623]).

Transbillayer movement of phospholipids in model lipid systems is a slow process [476, 545], obstructed by a high activation energy to pass the charged headgroup through the hydrophobic core of the lipid bilayer. Flipflop can be accelerated by introducing different physical properties between outer and inner monolayer, by insertion of bilayer spanning proteins, and by triggering the formation of non-bilayer structures in the lipid bilayer $[141,154]$. In erythrocytes, increased transbilayer movement is observed after reorganization of the interaction between cytoskeleton and plasma membrane $[15,39,95,103,194,217,218,423,477,526$, 703]. Previously, we have demonstrated that rapid flipflop during platelet activation is dependent on the degradation of cytoskeletal proteins by calpain $[124,663,664]$. Thus, transbilayer movement in activated platelets could very well be due to the generation of flipsites during calpain induced reorganization of the interaction between cytoskeletal proteins and plasma membrane components.

The occurrence of flipsites in the plasma membrane during platelet activation was investigated employing the fluorescent membrane probe TMA-DPH. This probe has been reported to incorporate quickly in the outer monolayer of the plasma membrane $[345,348]$. Due to a partition equilibrium [347], most of TMA-DPH will remain in solution, where it is hardly fluorescent [519]. Transbilayer movement of TMADPH [137] will result in labeling of intracellular compartments accompanied by more uptake of probe from solution which results in an increase in fluorescence intensity [347, 348]. Thus, the rationale was that loss of transbilayer asymmetry in platelets, which involves both inward and outward movement of phospholipids [50], would be accompanied by an increase in fluorescence intensity due to transport of TMADPH to intracellular membrane compartments. 


\section{MATERIALS AND METHODS}

\section{Platelet isolation}

Human platelets isolated, as outlined previously [51], by differential centrifugation from fresh blood drawn from healthy volunteers who received no medication. Blood was anticoagulated with acid-citrate-dextrose. Platelets were washed in an isotonic buffer at $\mathrm{pH} 6.6$, containing 10 muM Hepes (4-(2hydroxyethyl)-1-piperaxineethanesulfonic acid), $136 \mathrm{mM} \mathrm{NaCl}, 2.7 \mathrm{mM} \mathrm{KCl}, 2 \mathrm{mM}$ $\mathrm{MgCl}$, $5 \mathrm{miM}$ glucose (Hepes buffer) in the presence of $0.05 \%$ fatty acid-free human serum albumin. They were finally resuspended at a concentration of 2.108 per $\mathrm{ml}$ in Hepes buffer, pH 7.4, without albumin. Platelet concentration was inferred from their absorption at $405 \mathrm{~nm}$ using a calibration curve determined with a Coulter Counter. Platelet lysiss was determined by assaying lactate dehydro genase (EC 1.1.127) in a $12000 x \mathrm{~g}$ supermatant according to Wroblewski and La Due [710].

Totall platelet membranes were prepared by sonication in the presence of $1 \mathrm{mM}$ EGTA for $5 \mathrm{~min}$, with $10 \mathrm{sec}$ intervals, in a direct-probe sonicator (MSE) at an amplituide of $12 \mu \mathrm{m}$.

\section{Platelet lipid vesicles}

Total platelet lipids were extracted according to Bligh and Dyer [61], from platelets pooled from 8 donors. $\mathrm{Li}$ pids were stored in chloroform/methanol ( $1 / 1$, by voll.) at $-20^{\circ} \mathrm{C}$ under $\mathrm{N}_{2}$. Aliquots of lipid solutions were evaporated to dryness under a stream of $\mathrm{N}_{2}$. Dry lipids were hydrated by addition of Hepes buffer (pH 7.4, without albumin), which was degassed with N2. Vesicles were prepared by sonication. for $5 \mathrm{~min}$ at $40^{\circ} \mathrm{C}$, with an amplitude of $14 \mu \mathrm{m}$. After sonication, vesicles were centrifuged at $14.000 \mathrm{~g}$ for 5 min to remove non-vesicular structures. The final phospholipid concentration was determined according to Böticher etal [66], and varied between 2 and 5 mM. Cholesterol concentration was determined enzymatically by the cholesteroloxidase assay, using a commercial kit (Sigma, Si.Louis MO, USA).

\section{Platelet activation.}

Washed human platelets were diluted in quartix cuvettes at $37^{\circ} \mathrm{C}$ in Hepes buffer, $\mathrm{pH} 7.4$, containing $3 \mathrm{mM}$ $\mathrm{Ca}^{2+}$. At a concentration of $210^{7}$ per $\mathrm{ml}$, platelets were activated either by 2 nM thrombin (isolated and purified according to Rosing et.al. [544]) or $0.5 \mu \mathrm{M}$ ionomycin (Calbiochem, USA) under moderate stirring (250) rew/min). In dilution experiments, platelets were activated at a concentration of $210^{8}$ per $\mathrm{ml}$ by $4 \mathrm{nM}$ thrombin or 1 uM ionomycin in plastic tubes under otherwise similar conditions.

$\mathrm{Ca}^{2+}$ titration of intact platelets was carried out as described previously [664]. Extracellular free $\mathrm{Ca}^{2+}$ concentration was manipulated by a $30 \mathrm{mM}$ HEDTA/Ca ${ }^{2+}$ mixtures in Hepes buffer without magnesium. Intracellular $\mathrm{Ca}^{2+}$ was equilibrated with extracellular $\mathrm{Ca}^{2+}$ by addition of $0.5 \mu \mathrm{M}$ ionomycin to $2110^{7}$ plattelets per mi in quartz cuvettes at $37 \mathrm{C}$ under moderate stiirring: $\mathrm{All} \mathrm{Ca}^{2+}$ concentrations were checked with a $\mathrm{Ca}^{2}+$-selective electrode (Radiometer, Copenhagen, Sweden type $2110 \mathrm{Co}$

$\mathrm{Ca}^{2+}$-dependent actiwation of fuctide-treated platellets was perfiomed as outlined before $[663]$. Briefy, platelets were suspended to a concentration of $210^{8} \mathrm{per}$ ml in Hepes buffer, $\mathrm{pH} 7$, contaning 1 mM EOTA, and incubated with $1.0 \mathrm{mM}$ sodiumllooride (Merck, Damstad, FRG) without stirring at $37 \mathrm{C}$. At various time intervals subsarmple was diluted 10 times in Hepes buffer, $\mathrm{pH} 7.4$. and $5 \mathrm{mM} \mathrm{Ca} \mathrm{mas}^{2+}$ wasded.

\section{Fluorescence measurements}

TMA-DPH (Molecular Probes Incis Eugene OR, USA) was dissolved in $\mathrm{N}, \mathrm{N}$-dimethylformamide al a concentration of $5 \mathrm{mM}$ and stored at $20^{\circ} \mathrm{C}$ in the dark. Washed human platelets were diluted into a $3 \mathrm{ml}$ quartz cuvette with Hepes buffer $\mathrm{pH} 7.4$ to ta concentration of $210^{7}$ per mi and labeled at $37^{\circ} \mathrm{C}$ with 1 HM TMA-DPH under moderate stirring, using a double dilution procedure described in the previons chapter. Sonicated vesicles were employed at ancentration between 25 and $50 \mu \mathrm{M}$, vander the same conditions as platelets. All agents used during flworescence measurements were tested for their effect on the fluorescence properties of TMA-DPH, and were found to be without any effect unless explicitly mentioned.

Fluorescence intensities (F) were recorded at $37^{\circ} \mathrm{C}$ under moderate stirring with an SLM Aminco SPF-SOOC fluorimeter equipped with a light-tight injection port and a stirring device (Hellma). Excitation and emission wavelengths were set at 365 and $450 \mathrm{~nm}$ with 5 and $20 \mathrm{~nm}$ slits, respectively. F from light-scatter or background fuorescence varied between 1 and $2 \%$, and was neglected.

Phase fluorimetry was performed on a SLM -4800 fluorimeter according to Prendergast et al. [519], using a POPOP reference solution in order to remove any colordependent response of the delector [361]. Lifetimes from phase shifts $\left(\tau_{p}\right)$ were determined at a frequency of 30 $\mathrm{MHz}$ by a cross-correlation method using the computer program supplied by the manulacturer. Excitation was accomplished wising a monochromator set at $360 \mathrm{~nm}(0.5$ nm bandpass). Emmision was passed through two KV408 out-off filters (Schoti) beforidetection. Background Duorescence and light scalter amounted to less than $1 \%$ of total intensity and were neglected. All measturements were performed at $37^{\circ} \mathrm{C}$ willhout stirring.

Fluorescence decay and fluorescence anisotropy decay were determined with a laser-pulsed single photon counting fuorimeter, described in detail elsewhere $[284,285$, 286, 671]. TMA-DPH was excited at $310 \mathrm{~nm}$ by a fre. quency-doubled, synchronously pumped rhodamine 60 dye laser Emission was monilored above $450 \mathrm{~nm}$ through K45 band-pass filter (Balzers). All measurements were performed at $37^{\circ} \mathrm{C}$ withoul stirring.

\section{Analysis of time-resolved data}

Data were analyzed as described in the previous chapter. "The fluorescence decay of TMA-DPH was routinely analyzed by a biexponential function. It addition, a tetraexponential function, fitting from lower channel numbers, was used to include the lifetime of TMA-DPH in solution. 
The average fluorescence lifetime, $\langle\pi\rangle$, was calculated from the individual components from:

$$
\langle\tau\rangle=2 \alpha_{i} T^{2} / \Sigma \alpha_{i}
$$

The anisotropy decay function, $r(t)$, of TMA-DPH in membranes was interpreted within the framework of the "wobbling-in-cone" model introduced by Kinosita et. all. [320, 322]:

$$
r(t)=r_{\infty}+\left(r_{0}-r_{\infty}\right) e^{(-1 / \phi)}
$$

where $n t i s$ is the fundamental anisotropy, $r_{\infty}$ is the residual anisotropy al the end of the experiment (about $30 \mathrm{~ns}$ ) dis the apparent relaxation time.

Membrane fluidity as sensed by TMA-DPH; is expressed in a static and a dynamic component. The static component is expressed by the second-rank order parameter, s, and obtained from:

$$
s=\sqrt{\left(r_{\infty} / r_{0}\right)}
$$

The cone-angle, $\theta_{c}$, describing the range of acyl-chain motion in the "wobbling-in-cone" model, is determined from:

$$
S=1 / 2 \cos \theta_{c}\left(1+\cos \theta_{c}\right)
$$

The dynamic component is expressed by the modelindependent diffusion constant for rotation around an axis perpendicular to the probe symmetry axis, $D_{\perp}$, and is evaluated from:

$$
D_{\perp}=\left(\mathrm{r}_{0}-\mathrm{r}_{\infty}\right) /\left(6 \mathrm{ro}_{\phi}\right)
$$

assuming that the anisotropy decays exponentially to $[9]$.

To accommodate the frequent use of steady-state anisotropy, $r_{5}$, to describe changes in fluidity, $r_{5}$ was also derived using the Perrin-equation:

$$
r_{\mathrm{si}}=\mathrm{r}_{\infty}+\left(\mathrm{r}_{0}-\mathrm{r}_{\infty}\right) /(1+\pi / \phi)
$$

in which $q$ was substituted by the average fluorescence lifetime, $\langle\tau\rangle$, from the biexponential fit, which excludes any contribution of the ultrafast fluorescence of TMADPH in buffer. In atdition, steady-state anisotropy can be expressed as the degree of polarization, $\mathrm{P}_{\mathrm{n}}$, related to $\mathrm{r}_{\mathrm{g}}$ :

$$
P=3 r_{8} /\left(2+r_{s}\right)
$$

$S_{1}, D_{1}$, and $r_{s}$ were calulated with an average $r_{0}$ obtained from 96 experiments, as described in the previous chapter.

\section{RESULTS}

\section{Transbilayer movement of TMA-DPH during platelet activation.}

Addition of TMA-DPH to unstimulated platelets results in an immediate rise in fluorescence intensity (F) (Fig. 1A). This increase in $F$ is due to membrane-bound TMA-DPH, which has a much higher average fluorescence lifetime, $\langle\pi\rangle$, than TMA-DPH in aqueous solution [previous chapter]. After the initial rise of $F$, we consistently observed a moderate decline, leveling off to a stable signal within a few minutes. This drop in F during illumination is also observed when TMA-DPH is added to Hepes buffer only, which suggests that it is a property of TMA-DPH fluorescence itself. Rapid bleaching of TMA-DPH upon illumination has been observed with fluorescence microscopy $[80$, 348], and suggested to be due to photoisomerization during the excited state [173]. Therefore, we suggest that photoisomerization causes the decrease in $F$, and that this decline reaches a steady-state equilibrium under moderate stirring.

A few minutes after addition of TMA-DPH to platelets, more than $96 \%$ of total TMA-DPH is still in solution (Table IIII, $\alpha_{1}$ ). The membrane-bound TMA-DPH is most likely located in the outer leaflet of the plasma membrane, since upon sonication the amount of TMA-DPH in solution decreases further to about $82 \%$ (Table III), suggesting that most of the platelet membranes are not labeled immediately after addition of TMA-DPH to intact platelets. The increase in membrane-bound fraction of TMADPH is accompanied by a twofold increase in $F$ (Fig. 1A). It is emphasized that $F$ cannot be directly interpreted as number of fluorescent molecules, since $F$ is also dependent on $\langle\tau\rangle$. which decreases upon platelet sonication (Table II), indicating that the increase in $F$ is an underestimation of the increase in membranebound TMA-DPH. The suggestion that TMADPH is initially located solely in the outer monolayer of platelets, is supported by observations with fluorescence microscopy which show that TMA-DPH initially labels only the plasma membrane $[80,348]$, which may be interpreted as the outer monolayer considering the rapid 
equilibrium of TMA-DPH between membranes and aqueous solution. Thus, under the conditions employed, only the outer leaflet of the platelet plasma membrane is labeled with TMA$\mathrm{DPH}$, and any increase in F would indicate unlabeled membrane becoming available for binding by TMA-DPH.
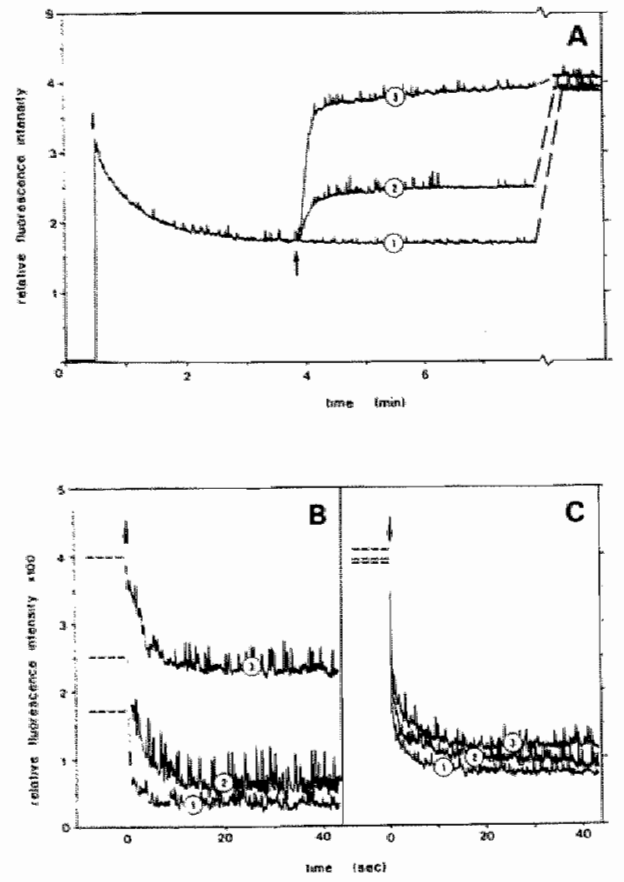

Fig. 1. Changes in membrane-bound pools of TMA-DPH during platelet activation.

(A): Washed human platelets were labeled with TMADPH in Hepes buffer in the presence of $\mathrm{Ca}^{2+}$ (thin arrow). After stabilization of the fluorescence signal, platelets lere left unstimulated (1), stimulated by $2 \mathrm{nM}$ thrombin (2) or by $0.5 \mu \mathrm{M}$ ionomycin (3), as indicated by the bold arrow. At the end of the measurement each platelet preparation was sonicated (dashed line), and $F$ was measured again using the same instrumental settings. $(B, C)$ : Subsamples from the very same platelet preparations used in panel A were 100 times diluted (arrow) in Hepes buffer without TMA-DPH, before (B) and after (C) sonication. The instrumental signal magnification was bundredfold increased with respect to the settings used for panel $A$. The dashed horizontal lines indicate $F$ of each platelet preparation before dilution, and represent $1 \%$ of $F$ in panel $A$, just before (B) or after (C) sonication. Trace numbers are the same as in panel $A$. Recordings shown ane representative for six experiments. All manipulations were carried out at $37 \mathrm{C}$ under moderate stirring. For further details is referred to the methods.
When platelets, which are labeled with TMA-DPH, are activated by $2 \mathrm{nM}$ thrombin, $\mathrm{F}$ increases about $45 \%$ (Fig. 1A). This has already been observed by Kubina etal. [345], who correlated this increase in $F$ with serotonin release, suggesting increased incorporation of TMA-DPH in the platelet plasma membrane due to exocytosis. Platelet lysis was $1.5 \%$, which is too small to account for the increase in F. Fluorescence decay analysis of TMA-DPH, added after platelet activation by thrombin, shows that the bound fraction of TMA-DPH increases from 3.6 to $5.6 \%$ (Table III). This increase is very well comparable to the increase in phospholipid hydrolysis in the outer monolayer of the platelet plasma membrane by exogenous sphingomyelinase and phospholipase $\mathrm{A} 2$ upon activation by thrombin (from 21 to $33 \%$ of total phospholipids) [50]. The similar increase in membrane-bound TMA-DPH and hydrolysable phospholip"ds in the outer leaflet of the plasma membrane upon platelet stimulation by thrombin supports the notion that the increase in $\mathrm{F}$ is solely due to an increased amount of plasma membrane lipids after fusion with granules. More conclusive evidence for the location of TMA-DPH in platelets after stimuilation of platelets by thrombin can be obtained from dilution experiments, which are based on the rapid equilibrium distribution of TMA-DPH between membranes and aqueous solution was employed. It was found that within $20 \mathrm{sec}$ after dilution of unstimulated platelets, F reached a new steady-state level (Fig. 1B). Thrombinactivated platelets reached a similar steadystate level of $F$ after dilution as unstimulated platelets, suggesting that TMA-DPH does not cross the plasma membrane during stimulation by thrombin. To verify whether stimulation by thrombin changes the TMA-DPH binding properties of the platelet plasma membrane, both unstimulated and thrombin-stimulated platelets were sonicated before dilution. No difference in steady-state level after dillution of the sonicate could be observed (Fig. 1C), indicating that the increase in $\mathbb{F}$ of TMA-DPH during stimulation of platelets by thrombin is not due to increased uptake capacity of the plasma membrane. Thus, the increase in $F$ upon activation of platelets with thrombin seems to be related only to exocytosis.

Activation of platelets by the $0.5 \mu \mathrm{M}$ ionomycin resulted in an increase in F of TMA-DPH of about $125 \%$ (Fig. 1A). This increase in $\mathrm{F}$ 
upon activation is much higher than with thrombin, and does not correlate with serotonin release [345]. Sonication of the platelets activated by ionomycin does hardly increase $F$ (Fig. 1A), which indicates that all platelet membranes become labeled by TMA-DPH during activation by the $\mathrm{Ca}^{2+}$ ionophore. Platelet lysis was $2.6 \%$. These findings strongly suggest that TMA-DPH labels all membranes within platelets during activation by ionomycin. Dilution of ionomycin-activated platelets reveals the presence of much larger non-dihtable pool of TMA-DPH compared to unstimulated or thrombin-activated platelets (Fig. 1B), which most likely results from penetration of TMA-DPH into platelets during stimulation by ionomycin. The increase in $\mathrm{F}$ and non-dilutable $\mathrm{F}$ during activation of platelets by ionomycin may, however, be due to increased uptake and possible irreversible binding of TMA-DPH in the ionomycin containing plasma membrane. This possibility can be excluded, however, since platelets sonicated after activation by ionomycin show a similar steady-state level of $F$ after dilution as do unstimulated platelets (Fig. 1C). Thus, it is concluded that flipflop of TMA-DPH over the platelet plasma membrane is the cause for the high levels of F observed after stimulation by ionomycin.

Flipflop of TMA-DPH in ionomycin-activated platelets may be caused by membrane disturbances induced by the presence of ionomycin itself. in the platelet plasma membrane. Ionomycin is applied at $0.5 \mu \mathrm{M}$, and at the platelet concentration used the phospholipid concentration was found to amount to $8 \mu \mathrm{M}$. Assuming that all ionomycin will dissolve into membranes, a phospholipid/ionomycin ratio of 16 clearly suggests effects of ionomycin on membrane order. The possibility that ionomycin itself affected lipid order was investigated using platelet lipid vesicles, to avoid complications due to cell activation. It was found that addition of ionomycin to a suspension of platelet lipid vesicles did increase lipid order (S) moderately (about 1\%), irrespective of the presence of $\mathrm{Ca}^{2+}$ or EGTA (Table I). Increase in lipid order has been suggested to be a general effect of molecules that dissolve in membranes [287]. Thus, it can be concluded that ionomycin itself does not decrease lipid order, suggesting that the structural integrity of the membrane is not impaired, and that rapid transbillayer movement of TMA-DPH in iono- mycin-activated platelets is caused by processes secondary to the addition of ionomycin.

\section{Alterations of the transbilayer nuidity gra- dient during platelet activation.}

Time-resolved fluorimetry revealed that, in addition to changes in $F$, platelet activation by thrombin resulted in an increased steadystate anisotropy of the plasma membrane: $r_{\mathrm{s}}$ increased from $0.260 \pm 0.005$ to $0.278 \pm 0.005$
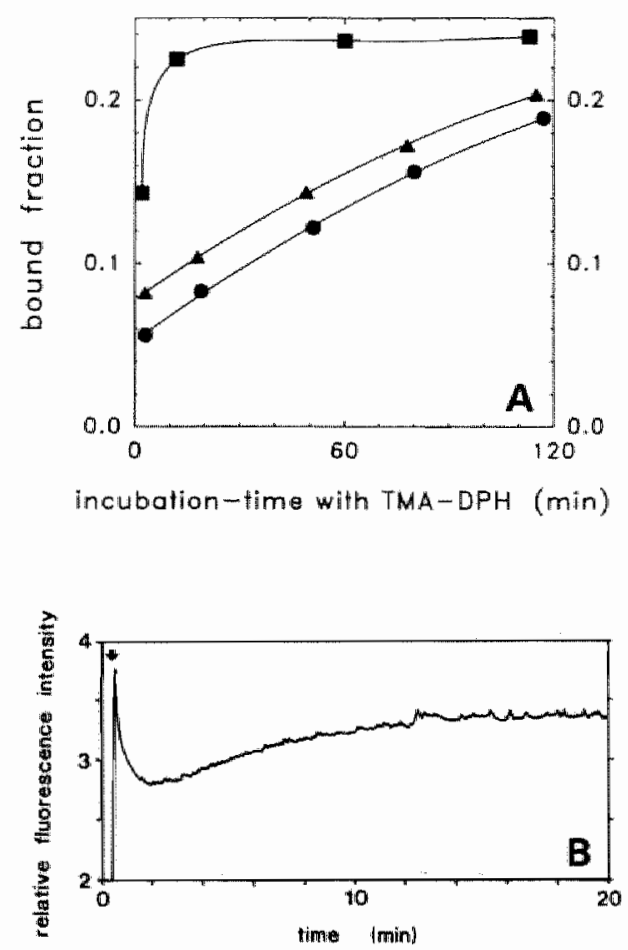

Fig. 2. Penetration of TMA-DPH into resting and activated platelets. (A) Platelets were activated as described in the methods. 1 HM TMA-DPH was added to unstimulated platelets (O), or two muinutes after activation by thrombin $(\boldsymbol{\Lambda})$ or ionomycin $(\boldsymbol{E})$. Only during stimulation and just before measurements, platelets were stirred. The fraction of TMA-DPH bound to platelets was determined as $\left(1-\alpha_{11}\right)$ from the tetraexponential fluorescence decay analysis. $(B)$ Fliptlop of TMA-DPH in ionomycin-activated platelets. TMA-DPH was added (arrow) two min after ionomycin. Experimental details are described in the methods. Data shown are representative for at least 3 experiments. 
(mean $\pm s . d ., n=5$ and $n=4$, resp.). This increase in $r_{s}$ is in good agreement with the work of Kubina et.al [345], who reported an increase in rs of TMA-DPH from 0.275 to 0.295 upon stimulation with thrombin. The increase in $\mathrm{I}_{\mathrm{s}}$ upon activation by thrombin was not accomparied by any change in the average lifetime of TMA-DPH, which remained $6.8 \pm 0.1 \mathrm{~ns}(\mathrm{n}=4)$. It is noted that these changes in fluidity and lifetime are confined to the outer monolayer of the platelet plasma membrane, considering the absence of transbillayer movement.

In contrast to thrombin, platelet activation by ionomycin resulted in a decrease in steadystate anisotropy from $0.260 \pm 0.005$ to $0.221 \pm$ 0.003 (mean \pm s.d., $n=5$ and $n=7$ resp.). Again, this is in good agreement with an earlier reported values of 0.275 and 0.240 , respective- ly [345]. The increase in fluidity was accompanied by a decrease in average lifetime from 6.8 \pm 0.1 to $5.1 \pm 0.1$ ns (mean $\pm s . d_{n, n}=5$ and $\mathrm{n}=7$, resp.), as determined by the biexponential fluorescence decay fit.

In wiew of findings presented in the previous chapter, which demonstrate the existence of a fluidity gradient over the plasma membrane of unstimulated platelets, it was of interest to find out what happens to this fluidity gradient during platelet activation. At first glance, it can be suggested from the steady-state anisotropies that the fluidity gradient is not much changed in thrombin-activated platelets, but must be reduced in ionomycin-activated platelets. Possible alterations of the fluidity gradient during platelet activation were investi-

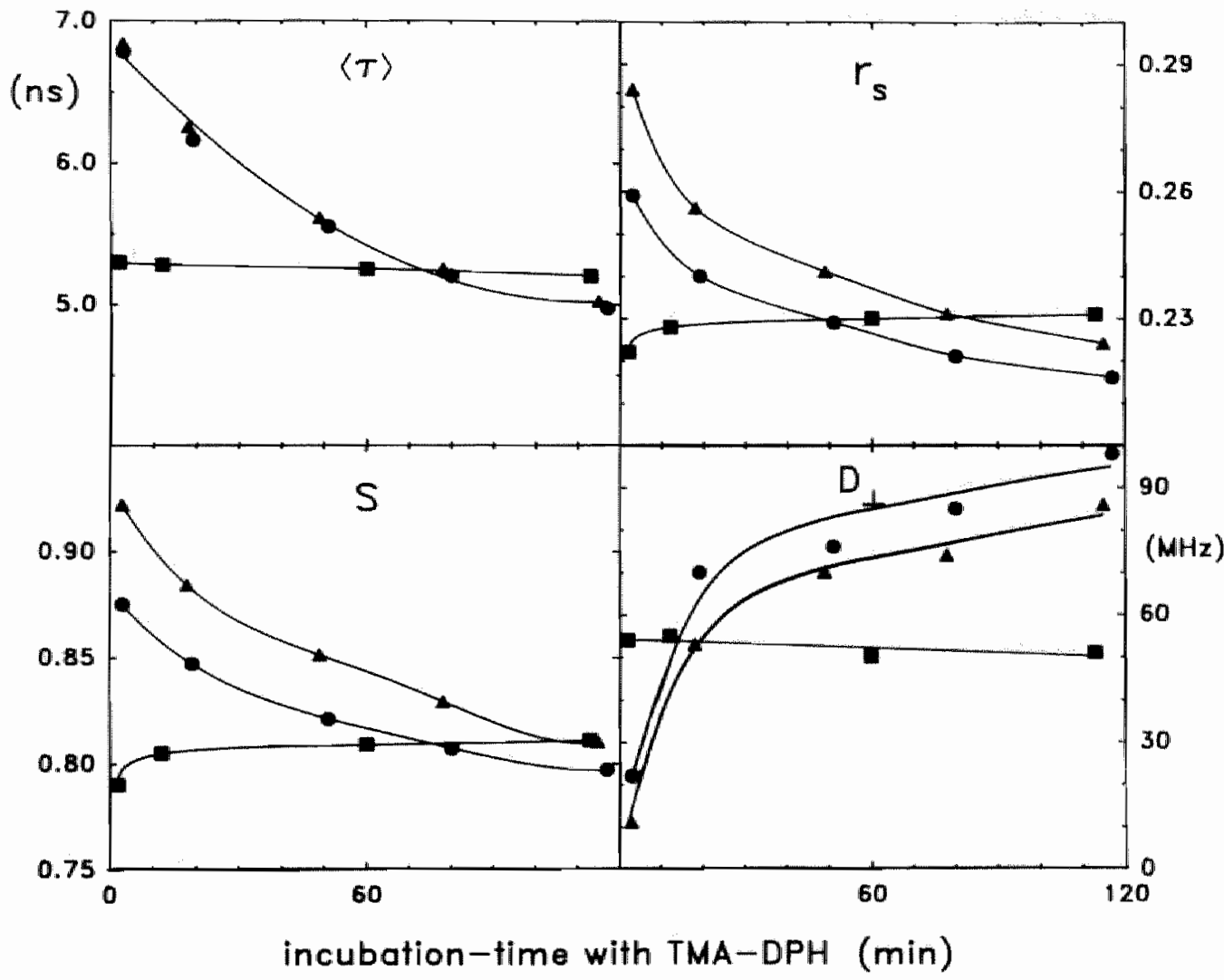

Fig. 3. Changes in lifetime and motional properties of TMA-DPH accompanying its penetration into resting platelets $(\boldsymbol{\theta})$ and platelets activated by thrombin $(\boldsymbol{\Delta})$ or ionomycin $(\boldsymbol{\omega})$ ). Presented are the average lifetime, $\langle\pi\rangle$, the steady-state anisotropy, $\mathrm{r}_{5}$, the structural order parameter, $\mathrm{S}_{\mathrm{x}}$ and the rotational diffusion constant, $D_{1}$. Experimental conditions are the same as in Fig. 2 . Data shown are representative for 3 experiments. 
gated by following the fluorescence properties of TMA-DPH during penetration into platelets.

First of all, the rate of penetration of TMADPH into activated platelets was compared with penetration into unstimulated platelets. It can be seen in Fig. 2, that uptake of TMA-DPH by thrombin-activated platelets follows a similar time course as uptake by unstimulated platelets, suggesting uptake by slow transbilayer movement. Uptake of TMA-DPH by ionomycinactivated platelets was quite different: after addition of TMA-DPH, the bound fraction quickly increased to a maximum levell within 15 to 20 min. This upper level is similar to that of sonicated platelets (Table $\mathrm{II}$ ), and seems to represent completely labeled platelets. Thus, it is suggested that flipflop of TMA-DPH over the platelet plasma membrane is considerably increased in ionomycin-activated platelets relative to unstimulated platelets and thrombin-activated platelets. The changes in $F$ after addition of TMA-DPH to ionomycin-activated pla telets (Fig. 2B), suggest that the fliprate of TMA-DPH in ionomycin-activated platelets is characterized by a t $1 / 2$ of about 10 minutes.

The effect of platelet activation on the fluidity gradient over the plasma membrane was examined by following the motional properties and the lifetime of TMA-DPH during penetration into activated platelets. It is shown in Fig. 3, that during prolonged incubation of thrombin-activated platelets, $\langle\tau\rangle$ decreased identically to unstimulated platelets. The motional properties of TMA-DPH in thrombinactivated platelets changed in a manner identical to unstimulated platelets, though the curve was shifted to higher order in thrombin-activated platelets, due to higher starting levels. The changes in motional properties of TMADPH during penetration into thrombin-stimulated platelets are characterized by a decrease in order (S) and an increase in rate of motion $\left(D_{\perp}\right)$ on a time scale of several hours. Thus, stimulation by thrombin does not alter the fluidity gradient in platelets.

Prolonged incubation of ionomycin-activated platelets with TMA-DPH showed completelly different results (Fig. 3). Lifetime as well as motional properties did not change much during incubation with TMA-DPH. $<\tau>$ and $D_{\perp}$ slowly decreased from 5.3 to $5.2 \mathrm{~ns}$ and from 54 to $51 \mathrm{MHz}$, respectively. Lipid order (S) and steady-state anisotropy $\left(r_{s}\right)$ increased from 0.79 to 0.81 and from 0.222 to 0.231 , re- spectively. It is emphasized that penetration of TMA-DPH in ionomycin-activated platelets is rapid: the final level is reached in about $20 \mathrm{~min}$ (Fig. 2B). During this time, the major part of the increase in $S$ and $r_{5}$ occurred (Fig. 3). Apparently, the fluidity gradient observed in unstimulated platelets is lost upon activation by ionomycin.

\section{Temporary character of flipsites.}

As already pointed out, after stimulation of platelets with ionomycin, transbilayer movement of TMA-DPH is enhanced with respect to unstimulated platelets: $t_{1 / 2}$ of flipflop decreases from about 1 hour (previous chapter) $1010 \mathrm{~min}$ (Fig. 2A). On the other hand, during stimulation by ionomycin, TMA-DPH becomes translocated into the platelet almost towards the maximum level within one minute (Fig. 1 and Table III). Thus, rapid flipflop of TMA-DPH during activation by ionomycin seems to be enabled only temporarily. This suggestion was further examined by comparing the fluorescence intensity of TMA-DPH, added before or after stimulation by ionomycin. Quantitative interpretation of differences in $\mathrm{F}$ after activation of platelets by either ionomycin or thrombin is allowed since the average lifetime is the same, both for addition of TMA-DPH after and before activation (Table II). It was found that when TMA-DPH was added one minute after ionomycin, $\mathrm{F}$ increased only about $40 \%$ above basal level instead of about $90 \%$ when TMA-DPH was added before ionomycin (Fig. 4A). Addition of TMA-DPH to platelets before or after thrombin did not make any difference (Fig. 4B).

The temporary character of rapid transbilayer movement of TMA-DPH during activation of platelets by ionomycin was further detailed by adding TMA-DPH at various time intervals after ionomycin. It is shown in Fig. $4 \mathrm{C}$ that during $30 \mathrm{sec}$ after addition of ionomycin the increase in F gradually drops. Addition of TMADPH more than $30 \mathrm{sec}$ after ionomycin did not result in lower levels of $F$ reached one minute after addition of TMA-DPH (not shown). Thus, it is suggested that rapid flipflop, as sensed by TMA-DPH, is present in ionomycin-activated platelets only for about $30 \mathrm{sec}$. 


\section{$\mathrm{Ca}^{2+}$-dependence of nipsites.}

Rapid transbilayer movement of TMA-DPH ower the platelet plasma membrane during activation by ionomycin, but not by thrombin, resembles the flipflop of phospholipids in its agonist-dependence $[50,124]$. We have shown previously that flipflop of phospholipids in activated platelets is dependent on the action of calpain towards cytoskeletal proteins $[124,663$, 664]. To investigate the involvement of calpain in the generation of flipsites during platelet ac-
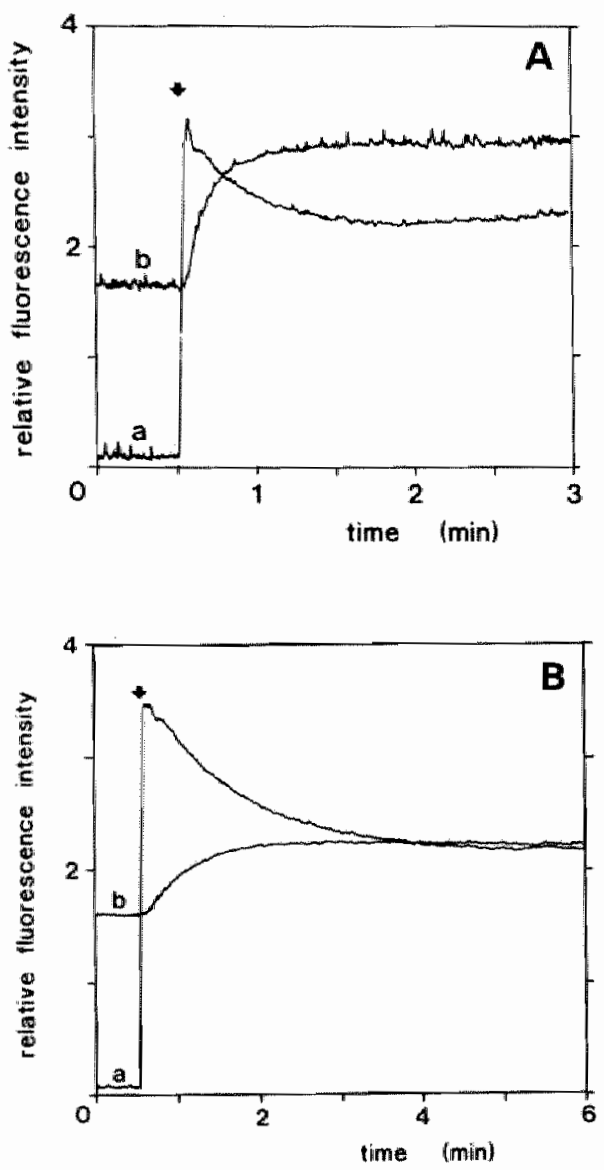

tivation by iononycin, we determined the $\mathrm{Ca}^{2+}$-dependence of fast transbilayer movement of TMA-DPH, following a previously described procedure [664]. Intracellular free $\mathrm{Ca}^{2+}$ was manipulated by applying buffered free $\mathrm{Ca}^{2+}$ levels outside and employing jonomycin to equilibrate intra- and extracellular free $\mathrm{Ca}^{2+}$ levels. For comparison with the $\mathrm{Ca}^{2+}$-dependence of platelet procoagulant activity and calpain [664], the development of $F$ upon addition of ionomycin was monitored for $5 \mathrm{~min}$.

Addition of ionomycin to platelets suspended in HEDTA/Ca ${ }^{2+}$ buffers resulted in a biphasic increase in fluorescence intensity of TMA-DPH (Fig. 5A). The first increase was observed with all $\mathrm{Ca}^{2+}$ levels, and correlates with the release reaction, which was found to be elicited by iononycin at any $\mathrm{Ca}^{2+}$ level. The second, and potentially much larger, increase in $F$ was dependent on free $\mathrm{Ca}^{2+}$ levels: at $1 \mu \mathrm{M}$ $\mathrm{Ca}^{2+}$ no additional increase in $\mathbb{F}$ was observed, while a maximal increase at 5 min after addition of ionomycin was obtained at $40, \mathrm{M} \mathrm{Ca}^{2+}$. Halfmaximal increase in $F$ was obtained at $\mathrm{Ca}^{2+}$ levels around $20 \mu \mathrm{M}$. This value is very similar to that at which halfmaximal calpain activity and flipflop of phospholipids has been observed in platelets [664].

Changes in fluorescence intensity of TMADPH do not necessarily indicate flipflop of

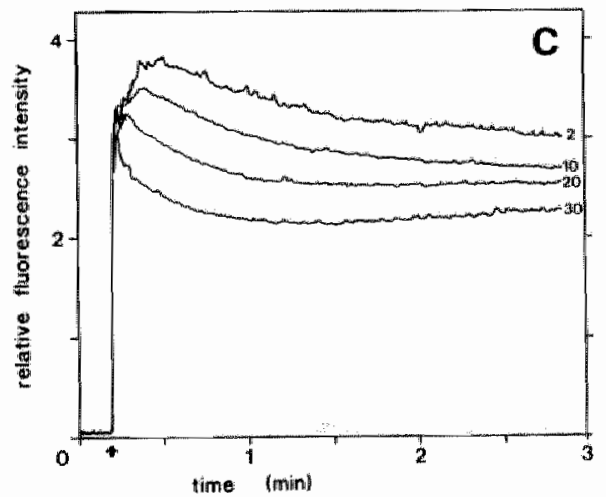

Fig. 4. Temporary character of lipsites in activated platelets. (A) Labeling of ionomycin-actiwated platelets with TMA-DPH, one minute after (a) or before (b) activation. Arrow indicates addition of TMA-DPH (a) or ionomycin (b). (B) Labeling of thrombin-activated plastelets with TMA-DPH, one minute after (a) or before (b) activation. Arrow indicates addition of TMA-DPH (a) or thrombin (b). (C) Labeling of ionomycin-activated platelets with TMA-DPH (arrow) at different times after activation, indicated in seconds by the numbers at the end of the recordings. Experimental details are described in the methods. Data shown are representative for 4 experiments. 
TMA-DPH. To confirm that the second increase in $\mathrm{F}$ upon addition of ionomycin to $\mathrm{Ca}^{2+}$-buffered platellets is due to transbilayer movement we determined the accompanying changes in lifetime, assuming, analogous to ionomycin-activated platelets, that rapid transbilayer movement of TMA DPH in platelets is accompanied by a loss of transbillayer fuidity gradient, resulting in alrop in fluorescence lifetime. Lifetimes were determined by phase-modulation fluorimetry before and 5 min after addition of ionomycin to $\mathrm{Cad}^{2+}$-buffered platelets. It was found
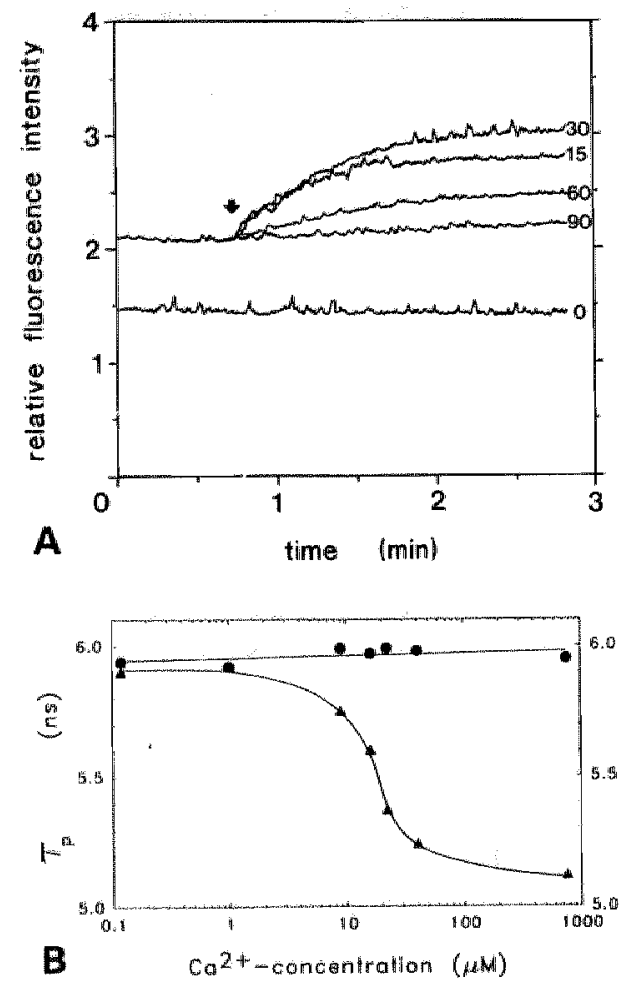

Fig. 5. $\mathrm{Ca}^{2+}$-dependence of nipflop of TMADPH during activation of platelets with $\mathrm{Ca}^{2+}$ lonophore. (A) Changes in fluorescence intensity of TMA-DPH alter addition of ionomycin (arrow) to platelets in the presence of various HEDTAVA ${ }^{2+}$ buffers. The extracellular free $\mathrm{Ca}^{24}$ concentration is indicated in ${ }_{\mu} \mathrm{M}$ at the end of the individual recordings. Data shown are representative for 5 experiments. (B) Changes in lifetime of TMA-DPH, as determined by phase-modulation fluorimetry $\left(\tau_{p}\right)$, after platelet activation by ionomycin in the presence of $\mathrm{Ca}^{2+}$ buffers. Lifetimes were determined two min after addition of TMA-DPH but one min before addition of ionomycin (e), and 5 min after addition of ionomycin to the same sample $(\boldsymbol{\Delta})$. Data slown are representative for 3 experiments. that the lifetime of TMA-DPH decreased with a similar $\mathrm{Ca}^{2+}$-dependence as the secondary increase in $\mathbb{F}$ (Fig. $5 B$ ), with a halfmaximal decrease around $20 \mathrm{M}$ free $\mathrm{Ca}^{2+}$. These findings suggest that the $\mathrm{Ca}^{2+}$-dependent increase in $\mathrm{F}$ is due to transbilayer mowement of TMA-DPH.

\section{$\mathrm{Ca}^{2+}$-dependent generation of nipsites in furoride-treated platelets.}

The $\mathrm{Ca}^{2+}$ - and time-dependence of rapid flipflop of TMA-DPH in ionomycin-activated platelets are similar as found for calpain activity [664], suggesting a relation between cytoskeletal degradation and generation of flipsites. Such a relation would have major mechanistic implications. Therefore, we further explored this suggestion, employing fluoride-treated platelets, which have been used before to demonstrate a relationship between calpain activity and transbilayer movement of phospholipids [663]. Platelets were treated with fluoride for two hours, during which the permeability of the plasma membrane towards $\mathrm{Ca}^{2+}$ is transiently enhanced. At several time points during incubation with fluoride, subsamples were labeled with TMA-DPH and extracellular $\mathrm{Ca}^{2+}$ was added to stimulate calpain to a level dependent on the prevailing $\mathrm{Ca}^{2+}$ permeability.

Fluoride-treated platelets displayed both a $\mathrm{Ca}^{2+}$-independent and $\mathrm{Ca}^{2+}$-dependent increase in fluorescence intensity. The $\mathrm{Ca}^{2+}$ independent increase occurred during the first 15 min (Fig. 6A), and can be explained by the release reaction which follows a similar time course during fluoride-treatment [663]. After 15 min the $\mathrm{Ca}^{2+}$-independent rise in $\mathrm{F}$ does not change anymore. The $\mathrm{Ca}^{2+}-$ dependent increase in $F$ varies during incubation with fluoride: during fluoride treatment its importance increases and around 30 min fluoride treatment the increase is about $50 \%$ relative to $\mathrm{Ca}^{2+}$-independent $F$, while after 90 min fluoride treatment addition of $\mathrm{Ca}^{2+}$ causes an increase of about $10 \%$ (Fig, 6A). This transient capacity of extracellular $\mathrm{Ca}^{2+}$ to induce an increase in $\mathrm{F}$ of fluoride-treated platelets labeled with TMADPH resembles the transient increase in $\mathrm{Ca}^{2+}$ permeability very well [663].

To investigate whether the $\mathrm{Ca}^{2+}$-dependent increase in $F$ is caused by transbillayer 
movement of TMA-DPH, we determined the accompanying changes in lifetime of TMA-DPH. It was found that addition of $\mathrm{Ca}^{2+}$ to fluoridetreated platelets caused a decrease in lifetime of TMA-DPH as measured by phase-modulation fluorimetry (Fig. 6B). This decrease was maximal around $30 \mathrm{~min}$ of incubation with fluoride, where the lifetime was reduced from 6.05 to 5.2 ns, which is comparable to the decrease observed in ionomycin-activated platelets (Fig. 5B). Similar to $\mathrm{Ca}^{2+}$-dependent changes in $\mathrm{F}$ in fluoride-treated platelets, the decrease in lifetime became progressively smaller upon prolonged incubation with fluoride. From the parallel time course of $\mathrm{Ca}^{2+}$-dependent increase in $\mathrm{F}$ and decrease in lifetime, it is con-
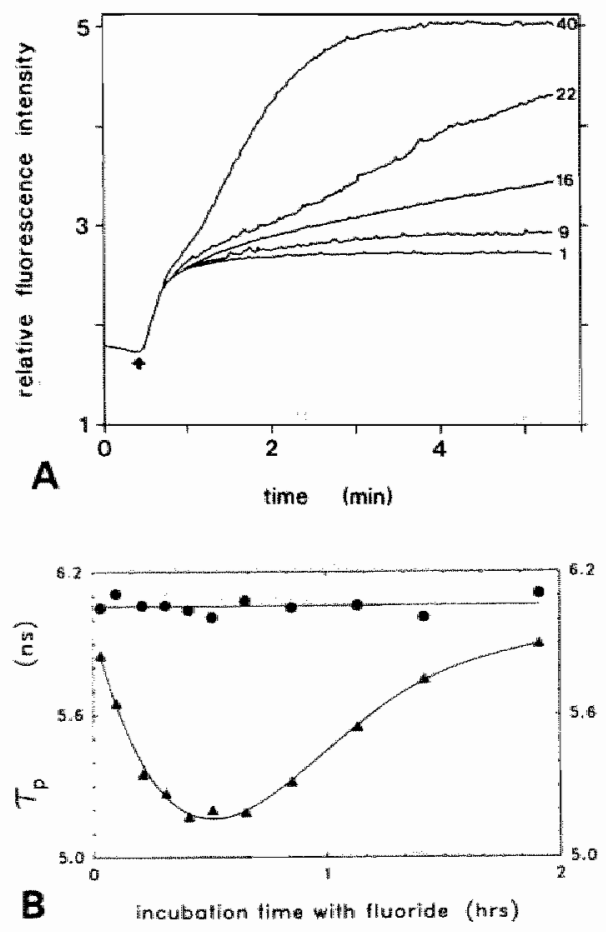

Fig. 6. $\mathrm{Ca}^{2+}$-dependent generation of flipsites in fluoride-treated platelets. (A) Changes in fluorescence intensity of TMA-DPH during incubation of platelets with fluoride, before and after addition of $\mathrm{Ca}^{2+}$ (arrow). Incubation time with fluoride is indicated in min at the end of the individual tracings. (B) Lifetime of TMA-DPH, as determined by phase-modulation fluorimetry $\left(\tau_{p}\right)$, in fluoride-treated platelets before $(\bullet)$ and after $(\Delta)$ addition of $\mathrm{Ca}^{2+}$. Experimental dictails are described in the methods. Data shown are representative for 3 experiments. cluded that tuoride-treated platelets transiently exhibit $\mathrm{Ca}^{2+}-$ inducible flipsites.

\section{DISCUSSION}

Transbilayer movement of TMA-DPH over the platelet plasma membrane appeared to be sensitive to changes in membrane organization found to occur during platelet activation, and a detailed insight in different properties of the flipsites, like their time- and $\mathrm{Ca}^{2+}$-dependence could be obtained. In addition, timeresolved fluorimetry revealed changes in membrane order as a result of the generation of hipsites during platelet activation. Throughout the investigations platelet activation by thrombin and by ionomycin were compared, because of their different potency to induce flipflop of PS in the platelet plasma membrane as reflected by generation of procoagulant activity: thrombin is hardly able to induce flipflop of PS, while the $\mathrm{Ca}^{2+}$ ionophore is the most potent stimulator known [50, 124, 664]. In accordance with their different potency in generating platelet procoagulant activity, major differences between thrombin and ionomycin were observed with respect to generation of flipsites.

Stimulation of washed human platelets by thrombin increased F of TMA-DPH, which can be directly interpreted as an increase in membrane-bound TMA-DPH, considering the absence of any change in lifetime. This increase in membrane-bound TMA-DPF has been clearly correlated with the release reaction [345]. Stimulation of platelets by thrombin was not accompanied by increased transbilayer movement of TMA-DPH. This is a surprising result for a relatively small and aspecific membrane probe, since it was expected that during fusion of granules with the plasma membrane flipflop of TMA-DPH would be enhanced due to disturbances of the lipid bilayer structure which necessarily occur during fusion. It is conceivable that actual fusion of membranes is too rapid to allow transbilayer movement of TMADPH, which will be limited by the lateral diffusion rate, or that fusilon involves too low a percentage of the total membrane to be detectable.

Stimulation of platelets by thrombin increased lipid order as sensed by TMA-DPH, 
while the lifietime of TMA-DPH was not changed. This is an unexpected result, since fusion with granules, which have a low cholesterol content $[243,556]$, would be expected to decrease $r_{s}$ in the platelet plasma membrane in view of the liquid-crystalline state of the plasma membrane at $37^{\circ} \mathrm{C}[623]$. Increased rigidity of the platelet plasma membrane upion actiwation by thrombin has been observed frequently, with various membrane probes $[345,450,451$, 613]. The increase in steady-state anisotropy of TMA-DPH in thrombin-activated platelets has been related to shape-change, and more specifically with the accompanying cytoskeletal reorganization [345]. However, it can be questioned whether the intracellular compartment is responsible for changes in lipid order as sensed by TMA-DPH, since TMA-DPH is confined to the outer leaflet of the plasma membrane in thrombin-activated platelets.

Generally, the most important membrane components which affect the fluorescence lifetime and anisotropy of TMA-DPH are SphM, cholesterol and proteins [chapter 5, Tables I and II]. The SphM content of the outer monolayer of the platelet plasma membrane is not affected by stimulation by thrombin [chapter 1 , Table II; 50], while any change in cholesterol content is likely to be a reduction due to fusion with granular membranes. Therefore, it is suggested that proteins are responsible for the observed increase in $r_{s}$, and not changes in the content of sphingomyelin or cholesterol in the outer monolayer of the plasma membrane. Using a variety of fluorescent probes, Kowalska and Cierniewski [336] demonstrated that binding of fibrinogen to ADP-activated platelets increased lipid order. Thus, we suggest that binding of fibrinogen, and possibly of released adhesive proteins like thrombospondin and fibronectin, to the surface of activated platelets is responsible for the increase in lipid order of the outer monolayer of the platelet plasma membrane observed after stimulation by thrombin.

Stimulation of platelets by ionomycin dramatically changed the lipid organization of the platelet plasma membrane. During activation by ionomycin TMA-DPH becomes subject to massive transbilayer movement. Such rapid and abundant flipflop most likely occurs along local disturbances of the lipid bilayer structure (flipsites), though direct or indirect inwolvement of membrane proteins cannot be excluded. Within one minute after activation by ionomycin these flipsites disappear. The time scale of rapid flipflop of TMA-DPH is similar to that of rapid flipflop of PS during generation of platelet procoagulant activity by the $\mathrm{Ca}^{2+}$ ionophore $\mathrm{A23187}$ [664]. Ionomycin was equally potent as A23187 in inducing flipflop of PS over the platelet plasma membrane as judged from dose-response relationships (unpublished results). Thus, it is suggested that during stimulation of platelets by ionomycin flipsites are generated, which provide a bidirectional means of transport, explaining the quick loss of phospholipid transbilayer asymmetry and the rapid flipflop of TMA-DPH.

It may be argued that incorporation of ionomycin into the plasma membrane can be responsible for rapid transbilayer movement by directly disturbing the lipid bilayer structure. This possibility can be excluded for the following reasons: (1) addition of ionomycin to platelet lipid vesicles increased lipid order, (2) continuous presence of ionomycin can not explain the temporary character of the flipsites, and (3) the effect of ionomycin on lipid order was independent of $\mathrm{Ca}^{2+}$, unlike the generation of flipsites.

Stimulation of platelets by ionomycin results in a loss of the transbilayer fluidity gradient. This can be explained by a randomization of lipids. Phospholipids randomize as a result of stimulation by a Ca ${ }^{2+}$ ionophore [50]. The loss of cholesterol transbilayer asymmetry as well, as inferred from the loss of the fluidity gradient, suggests a relation between the distribution of phospholipids and cholesterol. The preferential binding of cholesterol to SphM [158] could explain both the maintenance of cholesterol transbilayer asymmetry in unstimulated and thrombin-actiwated platelets, and the loss of this asymmetry in ionomycin-activated platelets.

Ionomycin-activated platelets display higher fliprates of TMA-DPH than unstimulated platelets, though still an order of magnitude lower than during stimulation by ionomycin. Transbilayer movement is influenced by the cholesterol content of the membrane: more cholesterol decreases the fliprate $[40,424]$. Apparently, the low fluidity of the outer monolayer in unstimulated platelets gives rise to low fliprates, which are considerably enhanced in ionomycin-activated platelets by the increase in fluidity of the outer leaflet of the plasma membrane.

Comparison of ionomycin-activated platelets with platelet lipid vesicles reveals that the 
motional properties of TMA-DPH are similar in both membrane systems (Table I). In addition, sonicated platelets show a decreased lipid order upon previous stimulation by ionomycin, relative to unstimulated platelets and thrombin-activated platelets. These findings suggest that the effect of proteins on platelet membrane fluidity is lost upon stimulation by ionomycin. In platelets treated with a $\mathrm{Ca}^{2}+$ ionophore, massive degradation of cytoskeletal proteins takes place $[124,664]$. Therefore, it is suggested that proteolysis by calpain abolishes the effect of membrane proteins on lipid order in the platelet membranes. This suggestion implicitly assumes that platelet cytoskeletal proteins interact with the lipid bilayer. Indeed, recent binding studies in our laboratory show that the platelet cytoskeleton (obtained as Triton insoluble residue) has a preferential affinity for PS (Comfurius et.al., unpublished results).

The experiments with thrombin- and ionomycin-activated platelets suggest a relationship between rapid flipflop of TMA-DPH, loss of transbilayer lipid asymmetry, and cytoskeletal degradation by calpain during stimulation of platelet procoagulant activity by ionomycin. Such a relation would strongly delineate mechanistic models explaining rapid flipflop of PS during platelet activation. Additional support for such a relation was obtained by examining the $\mathrm{Ca}^{2+}$-dependence of generation of flipsites during platelet activation by ionomycin, and by employing fluoride-treated platelets.

$\mathrm{Ca}^{2+}$ titration of the generation of flipsites in intact platelets showed a halfmaximal transbilayer movement of TMA-DPH at about $20_{\mu} \mathrm{M}$ free $\mathrm{Ca}^{2+}$, which is very similar to the $\mathrm{Ca}^{2+}$ dependence of PS-flipflop and calpain activity measured under the same conditions [664]. Flipflop of TMA-DPH is inferred from the combined increase in $\mathrm{F}$ and decrease in lifetime. This seems justified for the following reasons: either the decrease in lifetime is due to penetration of TMA-DPH into the platelet, or may reflect a change in the lipid composition of the outer leaflet of the plasma membrane, as seen in ionomycin-activated platelets. Both possibilities involve transbilayer movement. $\mathrm{Ca}^{2+}$ titration of flipflop of TMA-DPH shows that not only the maximal amount of fliptlop is $\mathrm{Ca}^{2+}$-dependent but the rate of transbilayer movement as well, suggesting that the rate of flipsite formation is determined by a $\mathrm{Ca}^{2+}-\mathrm{de}$ - pendent process and that the flipsites have a short lifetime.

Treatment of platelets with fluoride causes a transient increase in $\mathrm{Ca}^{2+}$ permeability of the platelet plasma membrane covering a period of two hours ${ }_{n}$ which was used to demonstrate a relationship between PS-flipflop and calpain activity [663]. Addition of $\mathrm{Ca}^{2+}$ to TMA-DPH labeled platelets after various times of fluoride treatment resulted in an increase in $F$ and a decrease in lifetime of TMA-DPH to an extent which followed the transient increase in $\mathrm{Ca}^{2+}$ permeability. These findings further support a relation between generation of flipsites, flipflop of $\mathrm{PS}_{\text {, }}$ and stimulation of calpain activity in platelets.

The findings presented in this paper suggest that flipflop of PS during expression of platelet procoagulant activity occurs via flipsites at which transbilayer movement is greatly enhanced, and that these flipsites are generated only during degradation of cytoskeletal proteins by calpain. A relation between calpain and flipsites can be understood in terms of a calpaindependent reorganization of the interaction between cytoskeletal proteins and lipids in the cytosolic leaflet of the plasma membrane. It is conceivable that the interaction between cytoskeletal proteins and membrane lipids contributes to the structural organization of the lipid bilayer, and that a loss of this interaction can result in a destabilization leading to a reorganization of the lipid bilayer. This suggestion is in line with general notions about interaction between cytoskeletall proteins and membrane lipids $[256,458]$, and is corroborated by ample observations relating the structural organiza tion of the cytoskeleton to that of the lipid bilayer of the plasna membrane in erythrocytes $[15,39,95,103,194,219,423,526,703]$ and in platelets [28].

\section{ACKNOWLEDGEMENTS}

Dr. Gert H.M. Kronenberg (Department of Plant Physiological Research, Agricultural University, Wageningen, Netherlands) is gratefully acknowledged for making phase-modulation fluorimetry available to us. We thank Dr. Arie van Hoek (Department of Molecular Physics, Agricultural University, Wageningen, 
Netherlands) for assistence with the laser fluo-

rimeter.

TABLE I. Fluorescence anisotropy decay parameters of TMA-DPH in various membranes.

\begin{tabular}{|c|c|c|c|c|c|c|c|c|c|c|c|c|}
\hline $\begin{array}{l}\text { membratane } \\
\text { preparation }\end{array}$ & $r_{0}$ & $r$ & $\stackrel{\phi}{(n)}$ & $x^{2}$ & DW & $Z_{\text {par }}$ & $Z_{\text {per }}$ & $r_{s}$ & $\mathrm{~S}$ & $\mathbf{P}$ & $\begin{array}{l}\theta_{c} \\
\left({ }^{\infty}\right)\end{array}$ & $\frac{\mathrm{D}_{1}}{\left(\mathrm{M} / \mathrm{H}_{2}\right)}$ \\
\hline $\begin{array}{l}\text { unstimulated } \\
\text { platelets }\end{array}$ & $\begin{array}{l}321 \\
.011\end{array}$ & $\begin{array}{l}.249 \\
.001\end{array}$ & $\begin{array}{r}1.08 \\
.114\end{array}$ & 1,08 & 1.88 & 218 & 259 & .258 & .884 & .343 & 229 & 34 \\
\hline $\begin{array}{l}\text { thrombin-act } \\
\text { platelets }\end{array}$ & $\begin{array}{l}302 \\
.005\end{array}$ & $\begin{array}{l}.267 \\
.001\end{array}$ & $\begin{array}{r}1.81 \\
.30\end{array}$ & .97 & 1.99 & 226 & 231 & .278 & 913 & 366 & 19.4 & 15 \\
\hline $\begin{array}{l}\text { thrombin-act } \\
\text { platelets }\end{array}$ & $\begin{array}{l}328 \\
.012\end{array}$ & .259 & $\begin{array}{r}1.04 \\
.14\end{array}$ & 1.03 & 1.89 & 223 & 264 & .267 & .903 & .353 & 20.9 & 30 \\
\hline $\begin{array}{l}\text { ionomycin-act } \\
\text { platelets A }\end{array}$ & $\begin{array}{l}.294 \\
.007\end{array}$ & $\begin{array}{l}.191 \\
.001\end{array}$ & $\begin{array}{r}1.33 \\
.10\end{array}$ & 1.07 & 1.86 & 216 & 234 & .217 & .775 & .294 & 32.6 & 50 \\
\hline $\begin{array}{l}\text { ionomycin act } \\
\text { platelets } 1 \mathrm{~B}\end{array}$ & $\begin{array}{l}.290 \\
.006\end{array}$ & $\begin{array}{l}.198 \\
.001\end{array}$ & $\begin{array}{r}1.35 \\
.10\end{array}$ & 1,04 & 1.89 & 200 & 244 & .223 & .788 & 301 & 31.6 & 47 \\
\hline $\begin{array}{l}\text { sconicate } \\
\text { unstimulated }\end{array}$ & $\begin{array}{l}329 \\
.006\end{array}$ & $\frac{.222}{.001}$ & $\begin{array}{r}1,10 \\
.07\end{array}$ & 1.05 & 1.88 & 181 & 200 & .241 & .836 & 322 & 27.6 & 46 \\
\hline $\begin{array}{l}\text { sonicate } \\
\text { thrombin-act }\end{array}$ & $\begin{array}{l}.3511 \\
.008\end{array}$ & $\begin{array}{l}.222 \\
.001\end{array}$ & $\begin{array}{r}1.07 \\
.07\end{array}$ & 1.08 & 1.95 & 260 & 252 & .239 & .836 & 321 & 27.6 & 47 \\
\hline $\begin{array}{l}\text { sonicate } \\
\text { fonomycin-act }\end{array}$ & $\begin{array}{l}314 \\
.007\end{array}$ & .210 & $\begin{array}{r}1.27 \\
.09\end{array}$ & 1.04 & 1.88 & 214 & 231 & 234 & .813 & 314 & 29.5 & 44 \\
\hline $\begin{array}{l}\text { platelet-lipid } \\
\mathrm{Ca}^{2+}\end{array}$ & $\begin{array}{l}326 \\
.005\end{array}$ & $\begin{array}{l}.203 \\
.001\end{array}$ & $\begin{array}{r}1,15 \\
.05\end{array}$ & $\mathbb{1 . 0 9}$ & 1.85 & 205 & 172 & .227 & .800 & 306 & 30.7 & 52 \\
\hline $\begin{array}{l}\text { platielet-lipid } \\
\text { EGTA }\end{array}$ & $\begin{array}{l}321 \\
.006\end{array}$ & $\begin{array}{l}.202 \\
.001\end{array}$ & $\begin{array}{r}1.10 \\
.06\end{array}$ & 1.05 & 1.96 & 202 & 239 & .225 & .796 & 303 & 31.0 & 56 \\
\hline $\begin{array}{l}\text { platelet lipid } \\
\text { ionomycan } \mathrm{Ca}^{2+}\end{array}$ & $\begin{array}{l}.336 \\
.007\end{array}$ & .2008 & $\begin{array}{r}1.04 \\
.06\end{array}$ & 1.06 & 1.87 & 180 & 233 & .229 & .808 & 308 & 30.1 & 56 \\
\hline $\begin{array}{l}\text { platelet-lipid } \\
\text { ionomycin/EGTA }\end{array}$ & $\begin{array}{l}3112 \\
.005\end{array}$ & $\begin{array}{l}.206 \\
.001\end{array}$ & $\begin{array}{r}1.28 \\
.07\end{array}$ & 1.02 & 1.90 & 212 & 256 & .231 & .804 & .310 & 303 & 46 \\
\hline
\end{tabular}

Fuorescence anisotropy decay was analysed with a single exponential and a residual anisotropy, as described in the methodks, starting at channel 54 (emmision peak at channel 26 , total number of channels is 1024, time-equivalence per channel was $325 \mathrm{ps}$ ). Standard deviations of fitted parameters are indicated underneath. Fit quality can be judged from $x^{2}$, the Durbin-Watson parameter (DW), and the number of zero passages in the autocorrelation function in the parallel $\left(Z_{\text {par }}\right)$ and perpendicular $\left(Z_{\text {per }}\right)$ direction. Activated platelets were either labeled 2 min after $(A)$ or 2 min before $(B)$ activation. In all cases, measurements were performed a few minutes after labeling. For further details is referred to the methods. Data shown are representative for at least 3 independent determinations. 
TABLE II. Biexponential fluorescence decay parameters of TMA-DPH in various membranes.

\begin{tabular}{|c|c|c|c|c|c|c|c|c|}
\hline $\begin{array}{l}\text { membrane } \\
\text { preparation }\end{array}$ & $\alpha_{1}$ & $\begin{array}{c}\tau_{1} \\
(\mathrm{~ns})\end{array}$ & $\alpha_{2}$ & $\begin{array}{c}T_{2} \\
(\mathrm{~ns})\end{array}$ & $\begin{array}{l}<\tau> \\
\text { (ns) }\end{array}$ & $x^{2}$ & DW & $\mathbf{Z P}$ \\
\hline $\begin{array}{l}\text { unstimulated } \\
\text { platelets }\end{array}$ & $\begin{array}{l}238 \\
.004\end{array}$ & $\begin{array}{r}2.39 \\
.08\end{array}$ & $\begin{array}{l}.762 \\
.005\end{array}$ & $\begin{array}{r}7.35 \\
.02\end{array}$ & 6.89 & 1.15 & 1.88 & 231 \\
\hline $\begin{array}{l}\text { thrombin-act } \\
\text { platelets }\end{array}$ & $\begin{array}{l}.232 \\
.004\end{array}$ & $\begin{array}{r}2.52 \\
.07\end{array}$ & $\begin{array}{l}.768 \\
.015\end{array}$ & $\begin{array}{r}7.28 \\
.02\end{array}$ & 6.83 & 1.04 & 2.00 & 224 \\
\hline $\begin{array}{l}\text { thrombin-act } \\
\text { platelets }\end{array}$ & $\begin{array}{l}.245 \\
.004\end{array}$ & $\begin{array}{r}2.45 \\
.07\end{array}$ & $\begin{array}{l}.755 \\
.005\end{array}$ & $\begin{array}{r}7.18 \\
.02\end{array}$ & 6.71 & 1.06 & 1.78 & 222 \\
\hline $\begin{array}{l}\text { ionomycin-act } \\
\text { platelets }\end{array}$ & $\begin{array}{l}350 \\
.005\end{array}$ & $\begin{array}{r}2.29 \\
.05\end{array}$ & $\begin{array}{l}.650 \\
.006\end{array}$ & $\begin{array}{r}5.73 \\
.02\end{array}$ & 5.12 & 1.16 & 1.74 & 197 \\
\hline $\begin{array}{l}\text { ionomycin-act } \\
\text { platelets } B\end{array}$ & $\begin{array}{l}345 \\
.004\end{array}$ & $\begin{array}{r}2,31 \\
.04\end{array}$ & $\begin{array}{l}.655 \\
.005\end{array}$ & $\begin{array}{r}5.69 \\
.01\end{array}$ & 5.110 & 1.08 & 1.88 & 204 \\
\hline $\begin{array}{l}\text { sonicated } \\
\text { unstimulated }\end{array}$ & $\begin{array}{l}388 \\
.003\end{array}$ & $\begin{array}{r}2.23 \\
.03\end{array}$ & $\begin{array}{l}.612 \\
.004\end{array}$ & $\begin{array}{r}6.14 \\
.01\end{array}$ & 5.41 & 121 & 1.69 & 170 \\
\hline $\begin{array}{l}\text { sonicate } \\
\text { thrombin-act }\end{array}$ & $\begin{array}{l}352 \\
.005\end{array}$ & $\begin{array}{r}2.57 \\
.05\end{array}$ & .648 & $\begin{array}{r}6.05 \\
.05\end{array}$ & 5.40 & 1.10 & 1.95 & 236 \\
\hline $\begin{array}{l}\text { sonicate } \\
\text { iomomycin-act }\end{array}$ & $\begin{array}{l}354 \\
.004\end{array}$ & $\begin{array}{r}2.20 \\
.04\end{array}$ & .646 & $\begin{array}{r}5.90 \\
.02\end{array}$ & 5,27 & 1.08 & 1.84 & 233 \\
\hline $\begin{array}{l}\text { platelet-lipid } \\
\mathrm{Ca}^{2+}\end{array}$ & $\begin{array}{l}382 \\
.004\end{array}$ & $\begin{array}{r}2.32 \\
.03\end{array}$ & .6005 & $\begin{array}{r}5.69 \\
.01\end{array}$ & 5.01 & 1.19 & 1.78 & 185 \\
\hline $\begin{array}{l}\text { platelet-lipid } \\
\text { EGTA }\end{array}$ & $\begin{array}{l}369 \\
005\end{array}$ & $\begin{array}{r}2.31 \\
.04\end{array}$ & $\begin{array}{l}.631 \\
.005\end{array}$ & $\begin{array}{r}5.66 \\
.02\end{array}$ & 5.01 & 1,08 & 1.98 & 176 \\
\hline $\begin{array}{l}\text { platelet-lipid } \\
\text { ionomycin/Ca }\end{array}$ & $\begin{array}{l}390 \\
.004\end{array}$ & $\begin{array}{r}2.25 \\
.03\end{array}$ & $\begin{array}{l}.610 \\
.005\end{array}$ & $\begin{array}{r}5.70 \\
.02\end{array}$ & 5.01 & 1.18 & 1.72 & 137 \\
\hline $\begin{array}{l}\text { platelet-lipid } \\
\text { ionomycin/EGTA }\end{array}$ & $\begin{array}{l}362 \\
.004\end{array}$ & $\begin{array}{r}2.29 \\
.04\end{array}$ & $\begin{array}{l}.638 \\
.005\end{array}$ & $\begin{array}{r}5.72 \\
.02\end{array}$ & 5.08 & 1,04 & 178 & 178 \\
\hline
\end{tabular}

Biexponential analysis of fluorescence decay was performed from channel 54 (emission-peak at channel 28). ZP: zero passages in the autocorrelation function. Standard deviations of fitted parameters are indicated underneath. Further details are described in Table I and the methods. Data shown are representative for at least 3 separate determinations. 
TABLE III. Tetraexponential nuorescence decay parameters of TMA-DPH in various membranes.

\begin{tabular}{|c|c|c|c|c|c|c|c|c|c|c|c|c|}
\hline $\begin{array}{l}\text { membrane } \\
\text { preparation }\end{array}$ & $\alpha_{1}$ & $\begin{array}{c}\tau_{1} \\
(\mathrm{~ns})\end{array}$ & $\alpha_{2}$ & $\begin{array}{c}\tau_{2} \\
\text { (ns) }\end{array}$ & $\alpha_{3}$ & $\begin{array}{l}T_{3} \\
(\mathrm{~ns})\end{array}$ & $\alpha_{4}$ & $\begin{array}{c}T_{4} \\
(\mathrm{~ns})\end{array}$ & $\begin{array}{l}<\pi> \\
(n s)\end{array}$ & $x^{2}$ & $\mathrm{DW}$ & $Z P$ \\
\hline $\begin{array}{l}\text { unstimulated } \\
\text { platelets }\end{array}$ & .064 & .038 & $\begin{array}{l}.004 \\
.001\end{array}$ & $\begin{array}{r}1.35 \\
.21\end{array}$ & $\begin{array}{l}.008 \\
.001\end{array}$ & $\begin{array}{r}4.15 \\
.50\end{array}$ & $\begin{array}{l}.024 \\
.001\end{array}$ & $\begin{array}{r}7.59 \\
.09\end{array}$ & 5.96 & 1.18 & 1.95 & 267 \\
\hline $\begin{array}{l}\text { Whrombin-act } \\
\text { platelets } A\end{array}$ & $\begin{array}{l}.944 \\
.003\end{array}$ & .038 & $\begin{array}{l}.004 \\
.002\end{array}$ & $\begin{array}{r}1.33 \\
.32\end{array}$ & $\begin{array}{l}.011 \\
.001\end{array}$ & $\begin{array}{r}3.35 \\
.42\end{array}$ & $\begin{array}{l}.041 \\
.001\end{array}$ & $\begin{array}{r}7.37 \\
.06\end{array}$ & 6.21 & 131 & 1.80 & 208 \\
\hline $\begin{array}{l}\text { thrombin-act } \\
\text { plattelets }\end{array}$ & $\begin{array}{l}.937 \\
.004\end{array}$ & .038 & $\begin{array}{l}.007 \\
.001\end{array}$ & $\begin{array}{r}1.18 \\
.17\end{array}$ & $\begin{array}{l}.014 \\
.001\end{array}$ & $\begin{array}{r}3.76 \\
.4 .2\end{array}$ & $\begin{array}{l}.043 \\
.002\end{array}$ & $\begin{array}{r}7.36 \\
.08\end{array}$ & 6.15 & 1.08 & 1.86 & 239 \\
\hline $\begin{array}{l}\text { ionomycin-act } \\
\text { platelets }\end{array}$ & .887 & .038 & $\begin{array}{l}.019 \\
.001\end{array}$ & $\begin{array}{r}0.83 \\
.06\end{array}$ & $\begin{array}{l}.040 \\
.002\end{array}$ & $\begin{array}{r}3.36 \\
.16\end{array}$ & $\begin{array}{l}.055 \\
.003\end{array}$ & $\begin{array}{r}6.02 \\
.07\end{array}$ & 4.78 & 1.24 & 1.75 & 214 \\
\hline $\begin{array}{l}\text { ionomycin-act } \\
\text { platellets }\end{array}$ & $\begin{array}{l}.840 \\
.006\end{array}$ & .038 & $\begin{array}{l}.027 \\
.002\end{array}$ & $\begin{array}{r}1.08 \\
.07\end{array}$ & $\begin{array}{l}.055 \\
.004\end{array}$ & $\begin{array}{r}3.51 \\
.18\end{array}$ & $\begin{array}{l}.079 \\
.005\end{array}$ & $\begin{array}{r}5.97 \\
.07\end{array}$ & 4.86 & 0.99 & 200 & 261 \\
\hline $\begin{array}{l}\text { sonicated } \\
\text { wnstimulated }\end{array}$ & $\begin{array}{l}.817 \\
.006\end{array}$ & .038 & $\begin{array}{l}.031 \\
.002\end{array}$ & $\begin{array}{r}0.99 \\
.07\end{array}$ & $\begin{array}{l}.062 \\
.002\end{array}$ & $\begin{array}{r}3.25 \\
.14\end{array}$ & $\begin{array}{l}.090 \\
.003\end{array}$ & $\begin{array}{r}6.41 \\
.05\end{array}$ & 5.21 & 1.12 & 1.85 & 245 \\
\hline $\begin{array}{l}\text { sonicate } \\
\text { thrombin-act }\end{array}$ & $\begin{array}{l}.813 \\
.008\end{array}$ & .038 & $\begin{array}{l}.019 \\
.004\end{array}$ & $\begin{array}{r}1.32 \\
.19\end{array}$ & $\begin{array}{l}.067 \\
.003\end{array}$ & $\begin{array}{r}3.46 \\
.24\end{array}$ & $\begin{array}{l}.108 \\
.006\end{array}$ & $\begin{array}{r}6.28 \\
.07\end{array}$ & 5.23 & 1.10 & 1.98 & 263 \\
\hline $\begin{array}{l}\text { sonicate } \\
\text { ionomycin-act }\end{array}$ & $\begin{array}{l}.829 \\
.008\end{array}$ & .038 & $\begin{array}{l}.029 \\
.004\end{array}$ & $\begin{array}{r}1.26 \\
.13\end{array}$ & $\begin{array}{l}.054 \\
.004\end{array}$ & $\begin{array}{r}3.47 \\
.31\end{array}$ & .088 & $\begin{array}{r}6.17 \\
.09\end{array}$ & 5.07 & 1.03 & 1.95 & 268 \\
\hline $\begin{array}{l}\text { platelet-lipid } \\
\mathrm{Ca}^{2+}\end{array}$ & $\begin{array}{l}504 \\
.014\end{array}$ & .038 & $\begin{array}{l}.078 \\
.004\end{array}$ & $\begin{array}{r}0.81 \\
.05\end{array}$ & .184 & $\begin{array}{r}3.19 \\
.11\end{array}$ & $\begin{array}{l}.235 \\
.009\end{array}$ & $\begin{array}{r}5.94 \\
.05\end{array}$ & 4.95 & 1.10 & 1.96 & 276 \\
\hline $\begin{array}{l}\text { platelet-lipid } \\
\text { EGTA }\end{array}$ & $\begin{array}{l}342 \\
.019\end{array}$ & .038 & .094 & $\begin{array}{r}0.75 \\
.06\end{array}$ & $\begin{array}{l}.232 \\
.007\end{array}$ & $\begin{array}{r}3.05 \\
.12\end{array}$ & $\begin{array}{l}332 \\
.012\end{array}$ & $\begin{array}{r}5.86 \\
.05\end{array}$ & 4.97 & 1111 & 1.96 & 239 \\
\hline $\begin{array}{l}\text { platelet-lipid } \\
\text { ionomycin/Cat }\end{array}$ & $\begin{array}{l}.405 \\
.017\end{array}$ & .038 & $\begin{array}{l}.098 \\
.005\end{array}$ & $\begin{array}{r}0.88 \\
.06\end{array}$ & $\begin{array}{l}.218 \\
.007\end{array}$ & $\begin{array}{r}3.116 \\
.11\end{array}$ & $\begin{array}{l}.279 \\
.010\end{array}$ & $\begin{array}{r}5.96 \\
.04\end{array}$ & 4.96 & 1.10 & 1.86 & 235 \\
\hline $\begin{array}{l}\text { platelel-lipid } \\
\text { lonomycin/EGTA }\end{array}$ & $\begin{array}{l}306 \\
1.020\end{array}$ & .038 & $\begin{array}{l}.099 \\
.007\end{array}$ & $\begin{array}{r}0.79 \\
.07\end{array}$ & $\begin{array}{l}.238 \\
.008\end{array}$ & $\begin{array}{r}3.07 \\
.14\end{array}$ & $\begin{array}{l}.358 \\
.014\end{array}$ & $\begin{array}{r}5.91 \\
.05\end{array}$ & 5.05 & 1.06 & 1.81 & 229 \\
\hline
\end{tabular}

Tetraexponential analysis started right at the emission peak, with $\tau_{\Perp}$ lixed at $38 \mathrm{ps}$. Standard deviations of fitted parameters are indicated underneath. Further details are described in Table I and the methods. Data shown are representative for at least 3 independent determinations. 


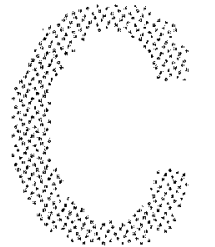

on
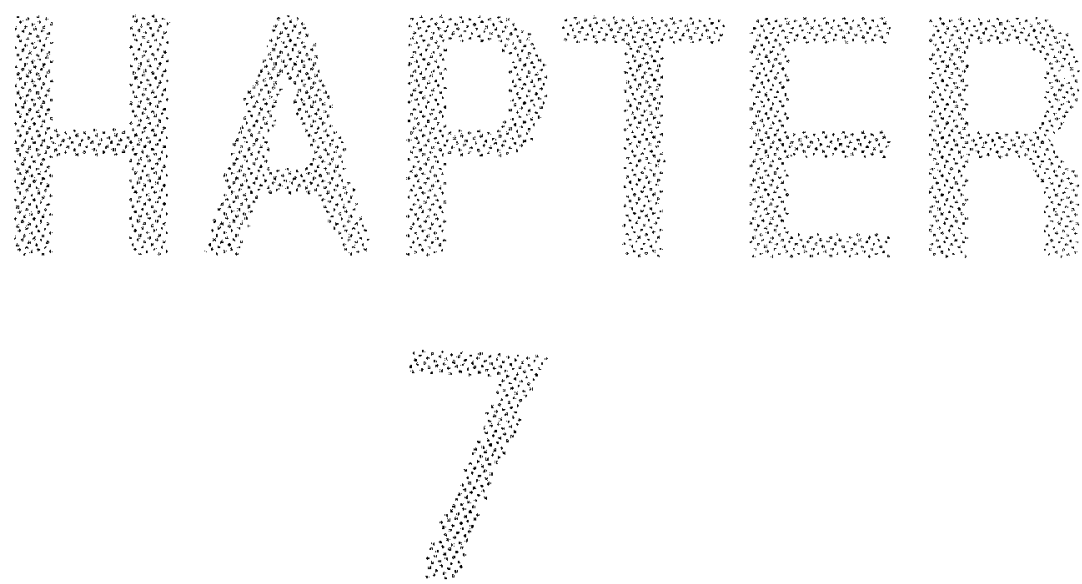


\section{GENERAL DISCUSSION}

\section{CELLULAR MECHANISMS OF PLATELET PROCOAGULANT ACTIVITY}

One of the major goals throughout the work presented in this thesis was to delineate the chain of cellular processes in platelets, from ligand-receptor interaction towards transbilayer movement (flipflop) of phosphatidylserine (PS) in the plasma membrane, rendering the platelet procoagulant. Here, an attempt will be made to highlight the key processes for the expression of platelet procoagulant activity.

A centrall role in the stimulation of platelet procoagulant activity is attributed to calpain. This is suggested by the ample correlations between calpain activity and procoagulant activity with respect to the time course of their development, their dose-response relationships, and their $\mathrm{Ca}^{2+}$-dependence (chapter 1 and 3 ). Most convincing, however, is the direct relation between calpain activity and procoagulant activity observed by the effect of leupeptin on both activities in fluoride-treated platelets (chapter 3). It is noteworthy that various unpublished observations further support this relation: incubation at $45^{\circ} \mathrm{C}$, depletion of metabolic ATP, and hypo-osmotic treatment, all increase platelet procoagulant activity accompanied by cytoskeletal degradation by calpain.

An intriguing question, pertinent to the evaluation of the importance of calpain in the expression of platelet procoagulant activity, is whether other cellular processes are also in- volved in the flipflop of PS. From the inability of thrombin to significantly increase platelet procoagulant activity beyond control levels (chapter 1), it can be concluded that normal routes of stimulus-response coupling in platelets are not sufficient to evoke flipflop of PS. This is also suggested by the appearance of procoagulant activity in fluoride-treated platelets, which is delayed with respect to aggregation and release (chapter 3 ). The ability of the local anesthetic dibucaine to render platelets procoagulant, suggests that phospholipase $\mathrm{A}_{2}$ and phospholipase $C$ dependent pathways are not necessarily involved in the processes leading to flipflop of $P S$, in view of the inhibition of both phospholipases and $\mathrm{C}$-kinase by dibucaine $[196,274,659,660]$. Thus, it is concluded that normal pathways of platelet stimulation are neither sufficient nor obligatory for the expression of platelet procoagulant activity. It may well be, however, that phospholipase $C$ and phospholipase $A_{2}$ dependent pathways play a facilitatory role in the generation of procoagulant activity under physiological conditions.

From chapters 2 and 3 it is obvious that elevation of cytosolic $\mathrm{Ca}^{2+}$ is a an important step in the stimulation of calpain. The $\mathrm{Ca}^{2+}$ titrations of unstimulated platelets suggest that, if $\mathrm{Ca} 2+$ would be the sole mediator, during stimulation of platelets by the combined action 
of thrombin and collagen, cytosolic $\mathrm{Ca}^{2+}$ levels $\left(\left[\mathrm{Ca}^{2+}\right] \mathrm{i}\right)$ of about $10 \mu \mathrm{M}$ would have to be reached, to explain the level of prothrombinase activity and cytoskeletal degradation (chapter 2). In general, $\left[\mathrm{Ca}^{2+}\right]$ increases upon platelet activation towards 1 to $2 \mu \mathrm{M}$ as measured by fluorescent $\mathrm{Ca}^{2+}$ chelators [263; chapter 1]. Thus, it may well be that expression of platelet procoagulant activity is dependent on higher $\left[\mathrm{Ca}^{2+}\right]_{i}$ than other $\mathrm{Ca}^{2+}$-dependent platelet processes. It has been argued in chapter 2 that the requirement for high $\mathrm{Ca}^{2+}$ levels can explain the necessity to use both thrombin and collagen to stimulate platelet procoagulant activity most significantly.

At present this suggestion is investigated in more detail by comparing $\left[\mathrm{Ca}^{2+}\right]_{i}$ in Fura2loaded platelets, reached upon stimulation by collagen and thrombin, either alone or in combination. Preliminary results are shown in Fig. 1. Stimulation of Fura2-labeled human platelets by thrombin results in a very fast increase in $\left[\mathrm{Ca}^{2+}\right] \mathrm{i}$ reaching a maximal value after $8 \mathrm{sec}$, followed by a gradual decline. This is in agreement with other observations on $\left[\mathrm{Ca}^{2+}\right]$ after stimulation by thrombin of Fura2-liabeled platelets $[263,509,525]$ and Quin2-labeled platelets $[13,60,264,303,535$, $641,680]$. Stimulation of platelets by collagen induced a small rapid increase in fluorescence, wich was found to be cue to collagen itself, followed by a gradual increase in $\left[\mathrm{Ca}^{2+}\right]_{\mathrm{i}}$ towards a maximal level. The delayed rise of $\left[\mathrm{Ca}^{2+}\right]_{i}$ upon stimulation by collagen has also been observed with Fura2-loaded platelets [510], Quin2-labeled platelets [14, 449] and aequorin-loaded platelets [448, 680]. Stimulation of platellets by the combined action of thrombin and collagen shows a rise in $\left[\mathrm{Ca}^{2+}\right]$ i that seems the sum of both agonist: a rapid rise to high levells followed by gradual increase reaching a maximal level after about one minute. Clearly, the maximal level is significantly higher than obtained by either agonist alone, though it is still lower than the $\left[\mathrm{Ca}^{2+} \mathrm{di}\right.$ reached after stimulation by lonomycin.

Apparently, thrombin and collagen increase $\left[\mathrm{Ca}^{2+}\right]$ by different mechanisms, resulting in different time courses. Generally, agonist-dependent elevation of $\left[\mathrm{Ca}^{2+}\right]_{i}$ in platelets involves both $\mathrm{Ca}^{2+}$ release from the dense tubular system and $\mathrm{Ca}^{2+}$ influx via divalent cation channels in the plasma membrane [263]. The importance of $\mathrm{Ca}^{2+}$ influx was investi- gated by stimulation of platelets in the presence of EGTA, which sequesters extracellular $\mathrm{Ca}^{2+}$ without interfering with $\mathrm{Mg}^{2+}$-dependent adthesion [558]. It was found that the rise in $\left[\mathrm{Ca}^{2+}\right]_{\mathrm{i}}$ by thrombin was greatly dependent on extracellular $\mathrm{Ca}^{2+}$, in that the maximal level was lower and the decline towards basal levels was more rapid than in the presence of extracellular $\mathrm{Ca}^{2+}$ (unpublished results). In contrast, the gradual rise of $\left[\mathrm{Ca}^{2+}\right]_{i}$ in collagen stimulated platelets was not dependent on

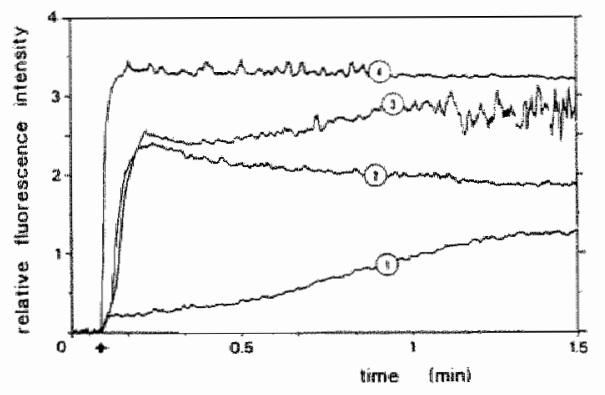

Fig. 1. Increase in cytosolic $\mathrm{Ca}^{2+}$ during stimulation of platelets by various agonists, as observed by Fura 2 fuorescence. Washed human platelets were isolated by differential centrifugation basically as described previously [82.BEVE.ECZ] from whole blood drawn from healthy volunteers receiving no medication. Platelets pelleted from platelet rich plasma were resuspended in Hepes buffer (10 $\mathrm{mM}$ Hepes (4(2hydroxyethyl)-1-piperazineethanesulfonic acid), $\mathrm{pH} 6.6$, $136 \mathrm{mM} \mathrm{NaCl}, 2.7 \mathrm{mM} \mathrm{KCl}, 2 \mathrm{mM} \mathrm{MgCl}, 5 \mathrm{mM}$ glucose and $0.05 \%$ fatty acid-free human serum albumine) to approximately $3 \times 10^{8} \mathrm{mal}^{-1}$ and incubated with $1 \mu \mathrm{M}$ Fura2-AM (Molecular Probes Inc, Eugene OR, U.S.A.) for $30 \mathrm{~min}$ at $37 \mathrm{C}$, following the procedure of Rao et.al. [85.RAO-,GPW]. After loading, platelets were washed twice in Hepes buffer, and were finally resuspended in Hepes buffer pH 7.4 at room temperature. Fluorescence measurements were performed at $2: 10^{7}$ platelets per $\mathrm{ml}$ in Hepes buffer pH 7.4 containing $3 \mathrm{mM} \mathrm{Ca}{ }^{2+}$. Fura2labeled platelets were stimulated by $(1) 20 \mu \mathrm{g} / \mathrm{ml}$ collagen (Hormon Chemie, Murich, F.R.G.), (2) 4 nM thrombin (isolated and purified from bovine blood according to Rosing et.al. [80.ROSIJTG]), (3) collagen plus thrombin, or (4) $0.5 \mu \mathrm{M}$ ionomycin (Calbiochem, U.S.A.) in stirred quartz cuvettes at $37^{\circ} \mathrm{C}$ under continuous monitoring of fluorescence intensity with a SLM Aminco SPF-500C fluorimeter equipped with a light-tight injection port. Excitation and emission wavelengths were set at 335 and $500 \mathrm{~nm}$ with 5 and $20 \mathrm{~nm}$ slits, respectively. Agonists were added (indicated by arrow) after setting basal Fura2 fluorescence to zero. Data shown are representative for 6 experiments. 
extracellular $\mathrm{Ca}^{2+}$ (unpublished results). The effect of EGTA on $\left[\mathrm{Ca}^{2+}\right.$ il during platelet activation by the combined action of thrombin plus collagen resembled that on $\left[\mathrm{Ca}^{2+}\right]_{i}$ during activation by thrombin only: a lower maximal level and a more rapid decline (unpublished results).

The dependence of $\left[\mathrm{Ca}^{2+}\right]$ in thrombinactivated platelets on extracellular $\mathrm{Ca}^{2+}$ is generally observed $[13,263,264,303,395,599 \mathrm{a}$, $680]$. The observation that $\left[\mathrm{Ca}^{2+}\right]_{i}$ in collagenstimulated platelets is independent of extracellular $\mathrm{Ca}^{2+}$ is not in agreement with other findings $[14,263]$. Stimulation of platelets by collagen is generally found to be dependent on secondary activation of non-adherent platelets by products of the cyclo-oxygenase pathway (thromboxanes, prostaglandin endoperoxides) formed by platelets adhering to collagen [263, $265 \mathrm{a}, 510,536 \mathrm{a}]$. In this way, the delayed increase in $\left[\mathrm{Ca}^{2+}\right] \mathrm{i}$ after stimulation by collagen is attributed to $\mathrm{Ca}^{2+}$ influx in platelets stimulated by thromboxanes [14, 448, 510]. However, pretreatment of Fura2-loaded platelets with aspirin, which irreversibly inhibits the cyclooxygenase, did not affect the rise in $\left[\mathrm{Ca}^{2+}\right]_{i}$ during stimulation by collagen and thrombin (unpublished results), in agreement with the lack of effect of aspirin on procoagulant activity [734]. The fact that $\left[\mathrm{Ca}^{2+}\right]_{i}$ in collagen-activated platelets, under our conditions, is not dependent on extracellular $\mathrm{Ca}^{2+}$ and not dependent on the cyclooxygenase pathway, can be explained by the high collagen to platelet ratio employed under stirring conditions. It is proposed that under these conditions most of the platelets will interact directly with collagen fibers, obviating the use of secondary activation by arachidonic acid products. This is supported by findings of others that, with increasing collagen concentrations, the effect of indomethacin or aspirin decreases $[557,680]$.

The observations with Fura2-labeled platelets are in line with the notion that $\mathrm{Ca}^{2+}$ is a key intracellular mediator of stimulation of platelet procoagulant activity: both calpain activity and procoagulant activity develop only after a delay of 60 to $90 \mathrm{sec}$ in platelets stimulated by the combined action of thrombin and collagen, and require the presence of extracellular $\mathrm{Ca}^{2+}$ (chapter 2). In addition, significant calpainand procoagulant activity in ionophore-activated platelets is observed only at relatively high $\left[\mathrm{Ca}^{2+}\right]_{i}$ of $10 \mu \mathrm{M}$ and higher (chapter 2 ).
Thus, it is concluded that maximal physiological stimulation of platelet procoagulant activity requires both collagen and thrombin, because the combination of both agonists generates the highest levels of $\left[\mathrm{Ca}^{2+}\right]$ i necessary for optimal generation of calpain and procoagulant activity.

Though mediators of platelet activation other than calpain may not be obligatory for the expression of platelet procoagulant activity, they may facilitate physiologicall stimulation of flipflop of PS. This is evident from the measurements with Fura2-labeled platelets (Fig. 1), which show that $1.5 \mathrm{~min}$ after stimulation by collagen, $\left[\mathrm{Ca}^{2+}\right]_{i}$ is still below $\left[\mathrm{Ca}^{2+} \mathrm{Ji}_{\mathrm{i}}\right.$ obtained after stimulation by thrombin. In contrast, collagen is a more potent agonist than thrombin for calpain activity as well as platelet procoagulant activity (chapter 1). Thus, $\mathrm{Ca}^{2+}$ independent mechanisms must operate in collagen-stimulated platelets to lower the $\mathrm{Ca}^{2+}$ dependence of calpain.

In view of the different potencies of thrombin and collagen in stimulation of platelet procoagulant activity, it can be argued that the $\mathrm{Ca}^{2+}$-independent mechanism must be specific for collagen. Thus, it is suggested that oollagen can modulate calpain activity independent of $\mathrm{Ca}^{2+}$, either by direct effects on calpain or by indirect effects like substrate modulation. $\mathrm{Ca}^{2+}$-independent modulation of calpain, by its phosphorylation, by modulation of calpastatin, or by the endogenous activator, is complex and still controversial [chapter 1]. On the other hand, phosphorylation of cytoskeletal proteins, as means for modulating their degradation by calpain has been obserwed on various occasions [512,645, 723]. At present it is not clear whether phosphorylation of cytoskeletall proteins in collagen-activated platelets is a plausible candidate for modulation of calpain activity, since the phosphorylation pattern is similar to that of thrombin-activated platelets [231]. Another $\mathrm{Ca}^{2+}$-independent mechanism whereby collagen may modulate calpain activity in platelets may be a specific structural reorganization of the membrane skeleton resulting from clustering of transmembrane proteins, imposed by the multi-valent interaction of collagen with transmembrane glycoproteins $[360,560$; chapter 1$]$.

Thusfar, only the route leading from platelet stimulation towards calpain activity has been adressed, leaving the route from calpain towards flipflop of PS to discuss in more detail. 
The most prominent action of calpain within platelets is degradation of the cytoskeletal proteins filamin, talin and myosin. It is emphasized that the action of calpain is certainly not limited to these proteins (see chapter 1). In addition, the involvement of calpain in expression of platelet procoagulant activity is judged indirectly from cytoskeletal degradation. Thus, it may be that substrates which constitute only a minor fraction of total platelet proteins are relevant for further propagation of the signal for nipflop of PS. However, transbilayer movement in ionophore-activated platelets results in dramatic changes in struicture and composition of the plasma membrane which are more likely to result from similarly dramatic changes in cytoskeletal proteins than from hydrolysis of minor platelet proteins. Moreover, cytoskeletal proteins have been implicated in the regulation of phospholipid transbilayer movement in the erythrocyte by direct interaction with the lipid bilayer (chapter 1). Thus, it is concluded that the role of calpain in the expression of platelet procoagulant activity is limited proteolysis of major actin-binding proteins, which will affect interactions of these proteins with the lipid bilayer of the plasma membrane, providing means for regulation of PS flipflop.

A submembraneous localization of cytoskeletal proteins would be a prerequisite for interactions with membrane lipids. In this respect, the major substrates of calpain in platelets, talin ,filamin, and myosin, have been shown to be present in a membrane skeleton preparation, isolated from resting platelets $[204,206]$ in considerable amounts. In general, membrane skeleton attachment is conducted by protein-protein linkage, involving actin barbed-end capping proteins $[136,229,684]$, like tallin [123], and actin-crosslinking proteins $[136,229$, 684], like filamin [542]. Filamin has been shown to bind to glycoprotein $\mathrm{l}_{\mathrm{b}}$ in resting platelets $[207,472,608,609]$. Anchorage of talin to platelet transmembrane proteins has not been demonstrated, though this is suggested by its presence in a platelet membrane skeleton preparation [204, 206]. In general, talin is found at sites of actin-membrane at-

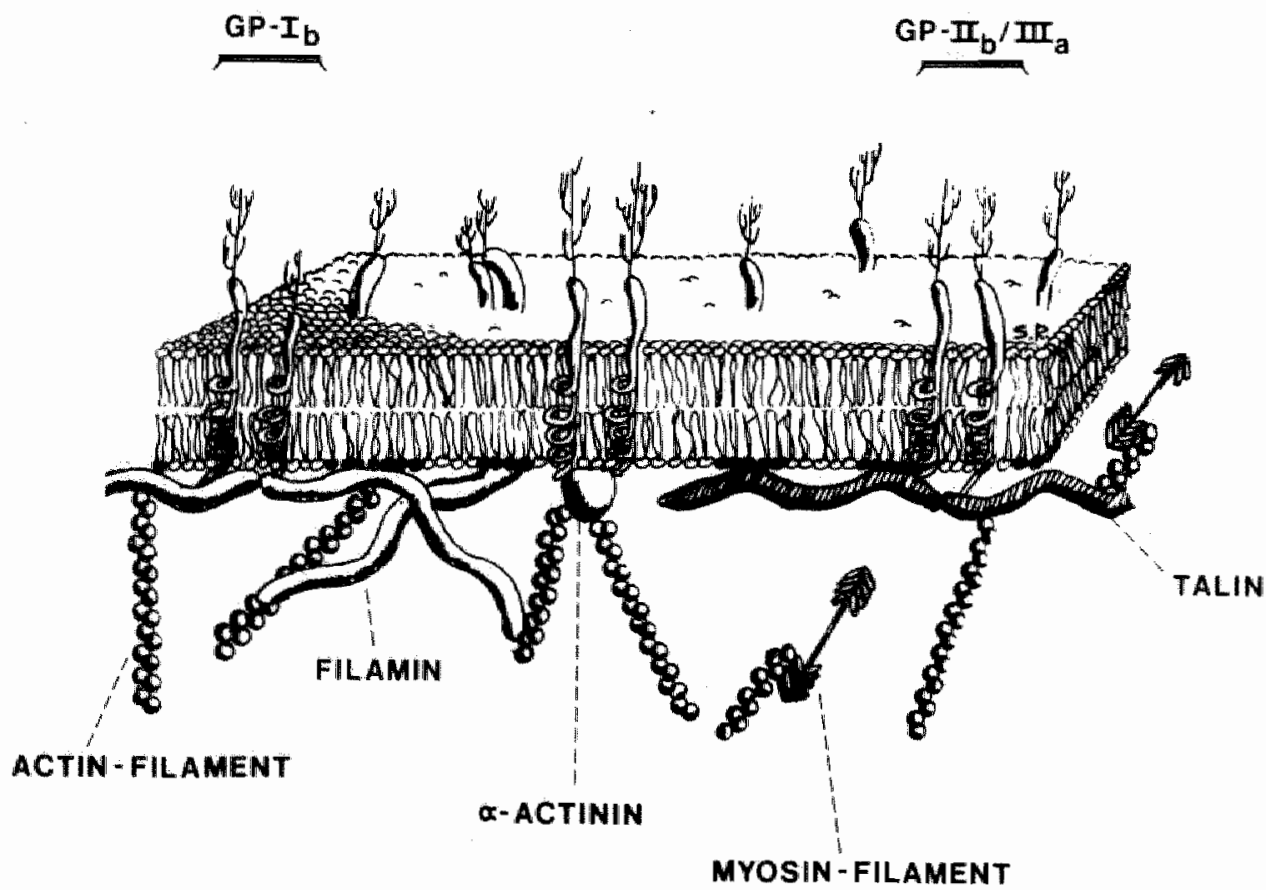

Fig. 2. The platelet membrane skeleton. Sichematic inpression based on current literature. The membrane skeleton is attached to membrane proteins and can directly interact with the lipid bilayer. The lipids of the membrane are magnified severalfold with respect to proteins in order to illustrate interactions between cytoskeletal proteins and anionic phospholipids, like phosphatidylserine (filled headgroups). 
tachment $[33,86]$. In fibroblasts and muscle cells, talin has been shown to bind to a complex of adhesive proteins, possibly belonging to a general class involved in adhesion, constituting a fibronectin receptor [292]. In platelets, the glycoprotein $\mathrm{IH}_{b} / \mathrm{II}_{\mathrm{a}}$ complex is implicated as the fibronectin receptor $[203,226,412 \mathrm{a}, 486 \mathrm{a}]$. Also in myoblasts calpain activation leads to changed properties of the fibronectin receptor [574]. The role of filamin and talin in mediating a link between the platellet cytoskeleton and transmembrane glycoproteins is in line with observations that local anesthetics decrease the ligand-binding capacity of glycoprotein Ib [119, $490,608]$ and the glycoprotein $\Pi_{b} / \Pi_{a}$ complex [120a, 490].

Thus, filamin and talin are implicated in the attachment of an actin-based membrane skeletal network to the platelet plasma membrane, by anchorage to glycoprotein $I_{b}$ and the glycoprotein $\Pi_{b} / \mathrm{III}_{\mathrm{a}}$ complex, respectively (Fig. 2). Clearly, such coupling proteins provide suitable means for manifesting interactions with membrane lipids suggested to be involved in the regulation of transbilayer asymmetry of PS. Additional candidates for interaction with

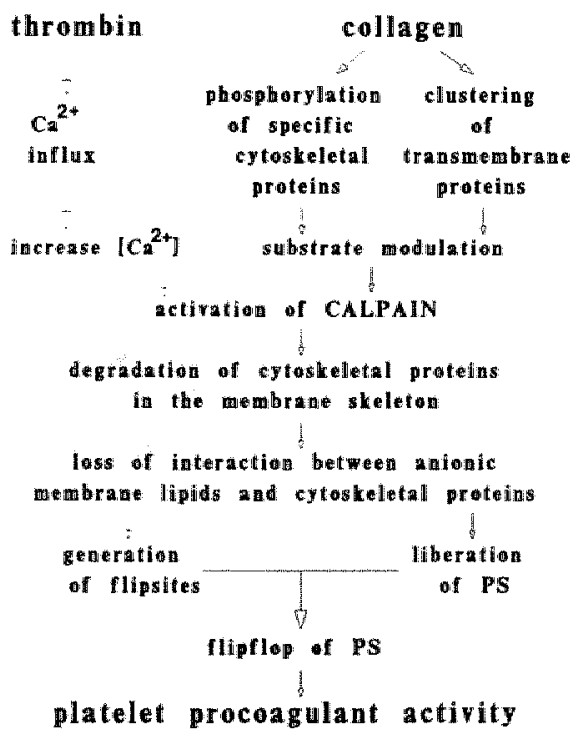

Fig. 3. Platelet cellular mechanisms inwolved in the expression of procoagulant activity upon stimulation by collagen and thrombin. Details are described in the text. membrane lipids are myosin and a-actinin, both substrates for calpain in platelets. Myosin in platelets is partly present in a submembraneous location [206,492], while a-actinin has been shown to bind to membrane lipids [85], especially diacylglycerol and fatty acids. Direet interaction between submembraneous cyloskeletal proteins with the lipid billayer may turn out to be a general phenomenon [458], and is best documented for the erythrocyte cytoskeletal proteins spectrin $[64,112,113,398,404,425$, 426] and band $-4.1[564,596]$. In platelets, the occurrence of direct interactions between membrane lipids and cytoskeletal proteins is demonstrated by recent binding studies at our laboratory, showing that platelet cytoskeletal proteins preferentially bind PS over phosphatidylcholine (PC) (Comfurius etal., unpublished results).

In summary, the following route of cellular mechanisms is suggested to be of major importance for the physiological stimulation of platelet procoagulant activity (Fig. 3). Both $\mathrm{Ca}^{2+}$ dependent and $\mathrm{Ca}^{2+}$-independent processes contribute to the degradation of cytoskeletal proteins by calpain. Thrombin and collagen are the most important stumuli for the $\mathrm{Ca}^{2+}-\mathrm{de}$ pendent and $\mathrm{Ca}^{2+}$-independent route, respectively. The importance of $\mathrm{Ca}^{2+}$ is evidenced by the potency of the $\mathrm{Ca}^{2+}$ ionophore and may explain the necessity of two strong platelet stimulators to obtain highest physiological stimulation of procoagulant activity. Influx of extracellular $\mathrm{Ca}^{2+}$ is needed to obtain sufficiently high levels of $\left[\mathrm{Ca}^{2+}\right] \mathrm{i}$ to stimulate calpain. As argued above, phosphorylation of cytoskeletal proteins or structural reorganizations of the membrane skeleton may be involved in the $\mathrm{Ca}^{2+}$-independent pathway, Alternatively, collagen may modulate calpain via its inhibitor (calpastatin) or its activator. Calpain-dependent degradation of cytoskeletal proteins, probably those which interact directly with the lipid bilayer of the platelet plasma membrane, will result in flipflop of PS from the intra- to the extracellular side of the plasma membrane, where PS is responsible for the platelet procoagulant activity. The molecular mechanisms of flipflop of PS will be discussed in the remainder of this chapter. 


\section{MOLECULAR MECHANISMS OF PLATELET PROCOAGULANT ACTIVITY}

A second major goal throughout the work presented in this thesis was to describe the molecular mechanisms at the level of the platellet plasma membrane which accompany or cause rapid transbillayer movement of PS. Application of the fluorescent membrane probe TMA-DPH, and the combination of fluorescence intensity and time-resolved fluorescence measurements, provided detailed information about the structural and dynamic properties of the platelet plasma membrane. It was found that platelets exhibit a considerable fluidity gradient over their plasma membrane (chapter 5), and that rapid flipflop of PS was accompanied by rapid transbilayer movement of TMA-DPH (chapter 6), suggesting that rapid flipflop in activated platelets occurs along local defects in the lipid bilayer structure (flipsites). It is the process by which these flipsites are formed and the role of cytoskeletal proteins therein which will be addressed in more detail.

To understand the molecular mechanisms governing the generation of flipsites, it is imperative to understand the molecular organization of the platelet plasma membrane first. The lipid bilayer of the platelet plasma membrane is characterized by an asymmetric distribution of its lipid components (Fig, 4A). SphM and PS are confined to the outer and inner monolayer, respectively, while $\mathrm{PC}$ and phosphatidylethanolamine (PE) are enriched in the outer and inner monolayer, respectively. Cholesterol [chapter 5] and polyunsaturated acyl chains [494] are predominantly located in the outer and inner monolayer, respectively. The asymmetric distribution of lipids over the platelet plasma membrane is accompanied by a considerable fluidity gradient over the membrane: the inner leaflet being much more fluid than the outer leaflet. A transmembrane fluidity gradient can be interpreted as a difference in lateral pressure between both monolayers of the membrane [222]. In general, however, membranes with different lateral pressures in their constituent leaflets are not stable lipid bilayers [ $154,164,549]$. Thus, the distribution of lipids over the platelet plasma membrane cannot be reconciled with a stable membrane, implicating involvement of other factors in the maintenance of membrane integrity in unstimulated platelets.

Stabilization of a biological membrane which contains an unstable lipid bilayer most likely is dependent on membrane proteins. These membrane proteins should annihilate the difference in lateral pressure between the two monolayers of the platelet plasma membrane. Therefore, transmembrane proteins are not likely candidates, considering their penetration through both monolayers. Peripheral membrane proteins [34, 161], which do not penetrate deeply into the lipid bilayer could provide a counterbalance for the fluidity gradient, thereby stabilizing the platelet plasma membrane. Shallow penetration of proteins into the lipid bilayer can be accomplished by an amphipathic helix $[176,189,190,299 \mathrm{a}, 370 \mathrm{a}$, $640,710 \mathrm{a}]$, in which hydrophylic and charged amino acid residues are located in the headgroup region where they can interact electrostatically with charged headgroups, and in which lipophilic amino acid residues are inserted into the bilayer interior where they interact hydrophobically with the acyl chains $[88,170]$ (Fig. 4B). Interaction of proteins with lipid monolayers can increase the lateral surface tension, as described for melittin and myelin basic protein [200], an $\mathrm{N}$-terminal fragment of adrenocorticotrophic hormone [665], spectrin $[398,425,426]$, apocytochrome c [501a], and plasma apolipoproteins [299].

In cells, cytoskeletal proteins are plausible candidates for a peripheral interaction with the cytosolic monolayer of the plasma membrane $[228,256,458]$. In platelets, a clwe about the occurrence of amphipathic helix-like interactions between peripheral membrane proteins and cytosolic membranes may be derived from the motional characteristics of TMA-DPH (chapter 5, Figs. 4 and 5). The rotational diffusion coeffi- 
cient $\left(D_{\perp}\right)$ of TMA-DPH follows a different course upon penetration of TMA-DPH into unstimulated platelets than fluorescence lifetime $(\langle\tau\rangle)$ and structural order $(S): D_{\perp}$ increases rapidly to a maximal value, while $\langle\tau\rangle$ and $S$ decrease gradually towards a lower limit. Generally, $D_{\perp}$ is inversily related to $S[320$, 620]. However, the value of $D_{\perp}$ obtained after equilibration of TMA-DPH with platelets (80 $\mathrm{MHz}$ ) is higher than these in egg- $\mathrm{PC}$ vesicles $(60 \mathrm{MHz})$, whille the value of $S(0.77)$ can be expected from a cholesterol/phospholipid ratio of 0.6 for the whole platelet (chapter 5, Fig. 6). The discrepancy between $S$ and $D_{\perp}$ of TMADPH within platelets can be explained by peripheral interaction of proteins with the mem- brane: shallow penetration of amino acids in the lipid bilayer would create a diserepancy between the extent of order in the headgroup region, which will dominate $S$ as sensed by TMADPI, and the rate of motion of acyl chains, which would not be restricted by the peripheral protein and which determines $D_{\perp}$ of TMADPH. An interaction between cytoskeletal proteins and lipid bilayer of the platelet plasma membrane is also suggested by the difference in fluidity between platelet membranes derived from unstimulated platelets and from ionophore-activated platelets; in which calpain has degraded the high-molecular weight actinbinding proteins (chapter 5). Thus, the motional behavior of TMA-DPH within platelets is
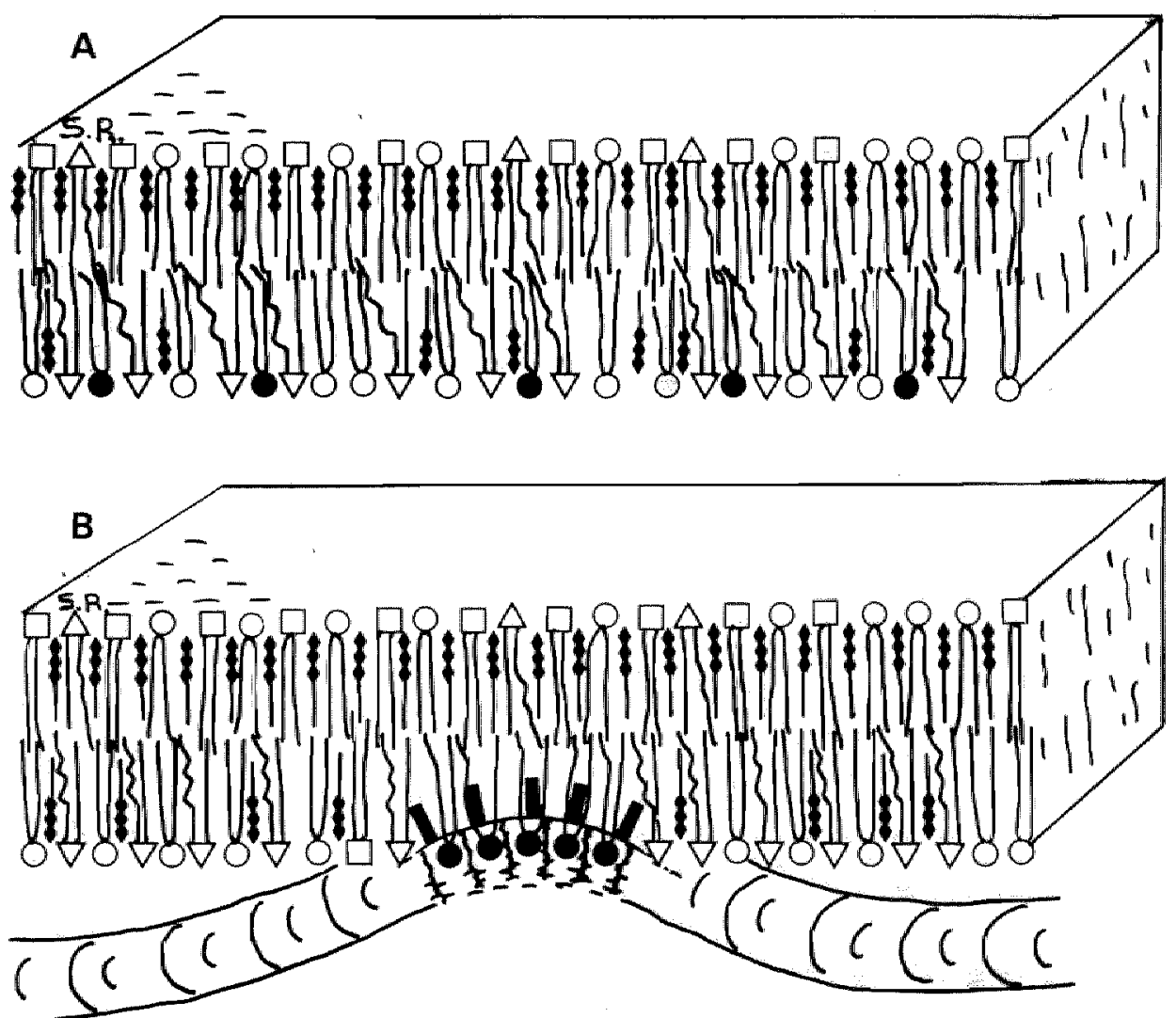

Fig. 4. Asymmetric distribution of lipids over the platelet plasma membrane (A), and the stabilization of this distribution by peripheral interaction between cytoskelletal proteins and the cytosolic leaflet (B), involving amphipathic helices. Phospholipids are identified by their headgroups as follows: SphM, ( $\square)$; PC, $(O) ; \mathrm{PE},(\triangle)$; PS, $(\bullet)$. Intercalated between the phospholipids is cholesterol identified by three chained filled diamonds. The filled rectangles in (B) represent hydrophobic amino-acid residucs penetrated into the the lipid bilayer. 
in line with the notion that amphipathic helixlike interactions between cytoskeletal proteins and the cytosolic leaflet of the plasma membrane occur within platelets.

Before elaborating possible mechanisms by which flipsites are generated in the platelet plasma membrane, it seems appropiate to consider the evidence for flipsites in more detail. In wiew of the use of TMA-DPH in the characterization of lipsites in the platelet plasma membrane, it can be questioned whether flipflop of TMA-DPH is representative for flipflop of phospholipids. Transbilayer movement of TMA-DPH in unstimulated platelets is characterized by an estimated halftime of 60 to $90 \mathrm{~min}$ (chapter 5). This fliprate can best be compared with fliprates of $\mathrm{PC}$, which is not subject to ac- tive transbilayer movement (chapter 1). The fliprate of TMA-DPH does not differ much from that of pyrene-PC in erythrocytes [224] and of short-chain spin-labeled PC in lymphocytes [722]. In general, however, fliprates of PC are several times lower than that of TMA-DPH in platelets, like the fliprate of PC $[218,413,643]$, lyso-PC [40,423], or short-chain spin-labeled PC [585] in erythrocytes. These results suggest that fliprates of TMA-DPH are an overestimation of fliprates of phospholipids, but still are in the same order of magnitude.

The fliprate of TMA-DPH during generation of platelet procoagulant activity is characterized by an estimated halftime of 15 to $20 \mathrm{sec}$ (chapter 6), which is two orders of magnitude faster than the fliprate in unstimulated plate-
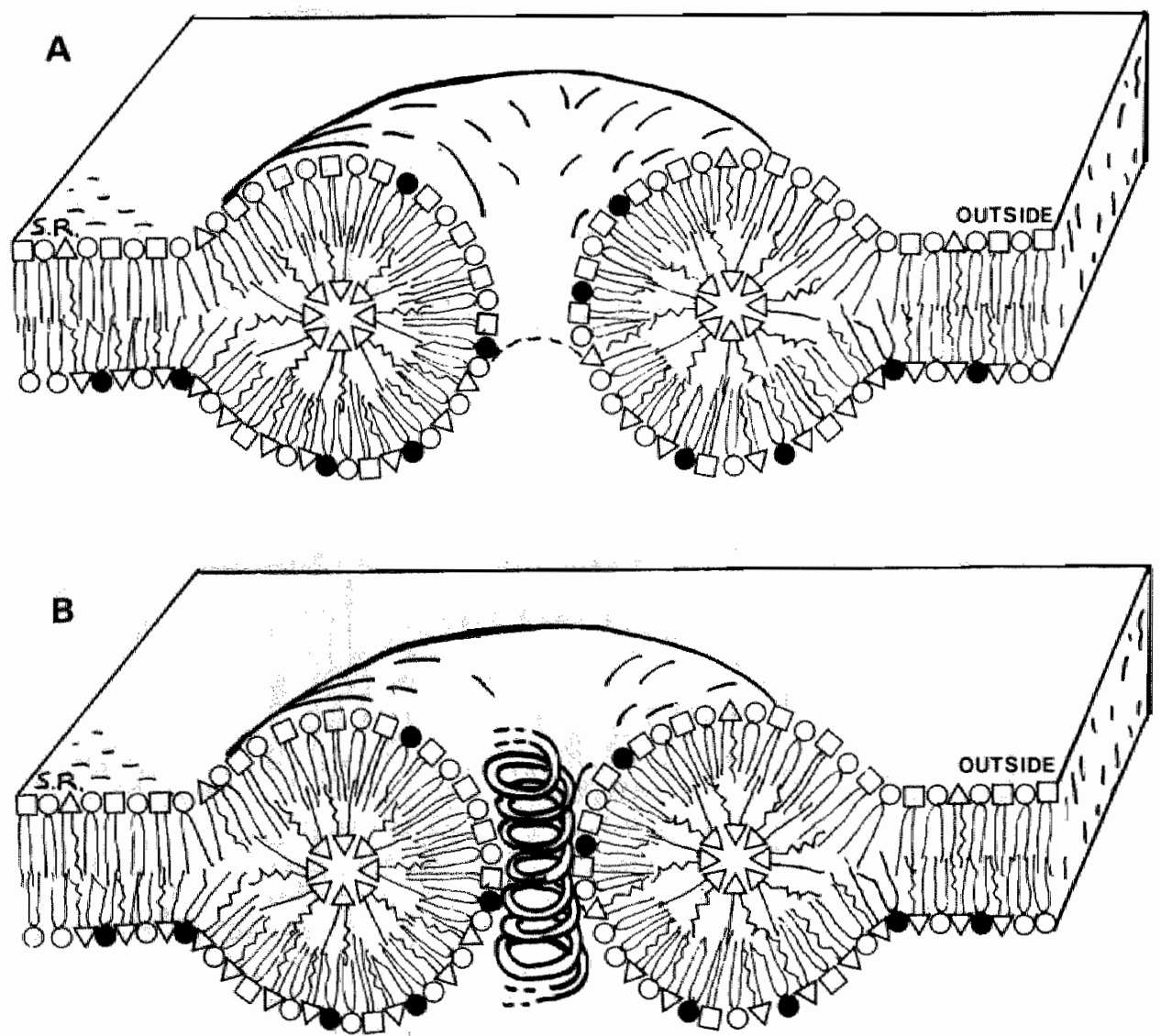

Fig. 5. Pore model for fipsites in the plasma membrane of activated platelets. The wall of the pore may be lined by hexagonal phase lipid $(A, B)$ or not $(C, D)$. The pore may be lipidic in nature $(A, C)$, or may contain a central transmembrane protein (B, D). Phospholipids are identified by their headgroup symbol, as explained in Fig. 4. Cholesterol is left out of the model for convenience. 
lets, and which is similar to the fliprate of PS in ionophore-activated platelets (chapter 2). As already argued in the introduction of chapter 6 , active transbilayer movement of TMA-DPH by a translocase must be excluded considering its specificity for the glycerophosphoryl backbone of PE and PS [406]. Exclusion of active transport leaves either passive or facilitated diffusion. Passive diffusion through an intact lipid bilayer could possibly explain the fliprate of TMA-DPH observed in unstimulated platelets $\left(t_{1 / 2} 60-90 \mathrm{~min}\right)$ and platelets after ionomycin activation ( $\left.\mathrm{t}_{1 / 2} 10 \mathrm{~min}\right)$, but not for the very high fliprates during ionomycin activation $\left(t_{1 / 2} 20\right.$ sec). Thus, the rapid flipflop of TMA-DPH during platelet acivation by ionomycin is explained by transbilayer movement facilitated by flipsites.

To understand the molecular nature of flipsites it is illuminating to summarize observations made with model lipid systems. Flipflop of phospholipids in unilamellar lipid vesicles is extremely slow under equilibrium conditions, i.e. in the absence of any physical or chemical transbilayer asymmetry, with halfimes of seweral weeks $[476,545]$. Fliplop in lipid wesicles can be increased in the following ways: (1) by an asymmetric distribution of phospholipids [339]; (2) by introduction of a transbilayer fuidity gradient [343]; (3) by packing defects at the boundary of gel-liquid crystalline phases [344]; (4) by formation of non-bilayer lipid structures $[141,234]$; (5) by introduction of transmembrane proteins $[233,612,726]$. From this summary, it is clear that transbilayer asymmetry of both phospholipids and fluidity over the platelet plasma membrane constitutes an important driving force for transbilayer movement. Nevertheless, passive transbilayer movement in unstimulated platelets is slow, judged by flipflop of PC [623]. Thus, rapid flipflop, as observed during platelet activation by ionomycin, must depend on the generation of struc tural defects of the lipid bilayer.

Limiting conditions describing the flipsite in activated platelets in more detail can be
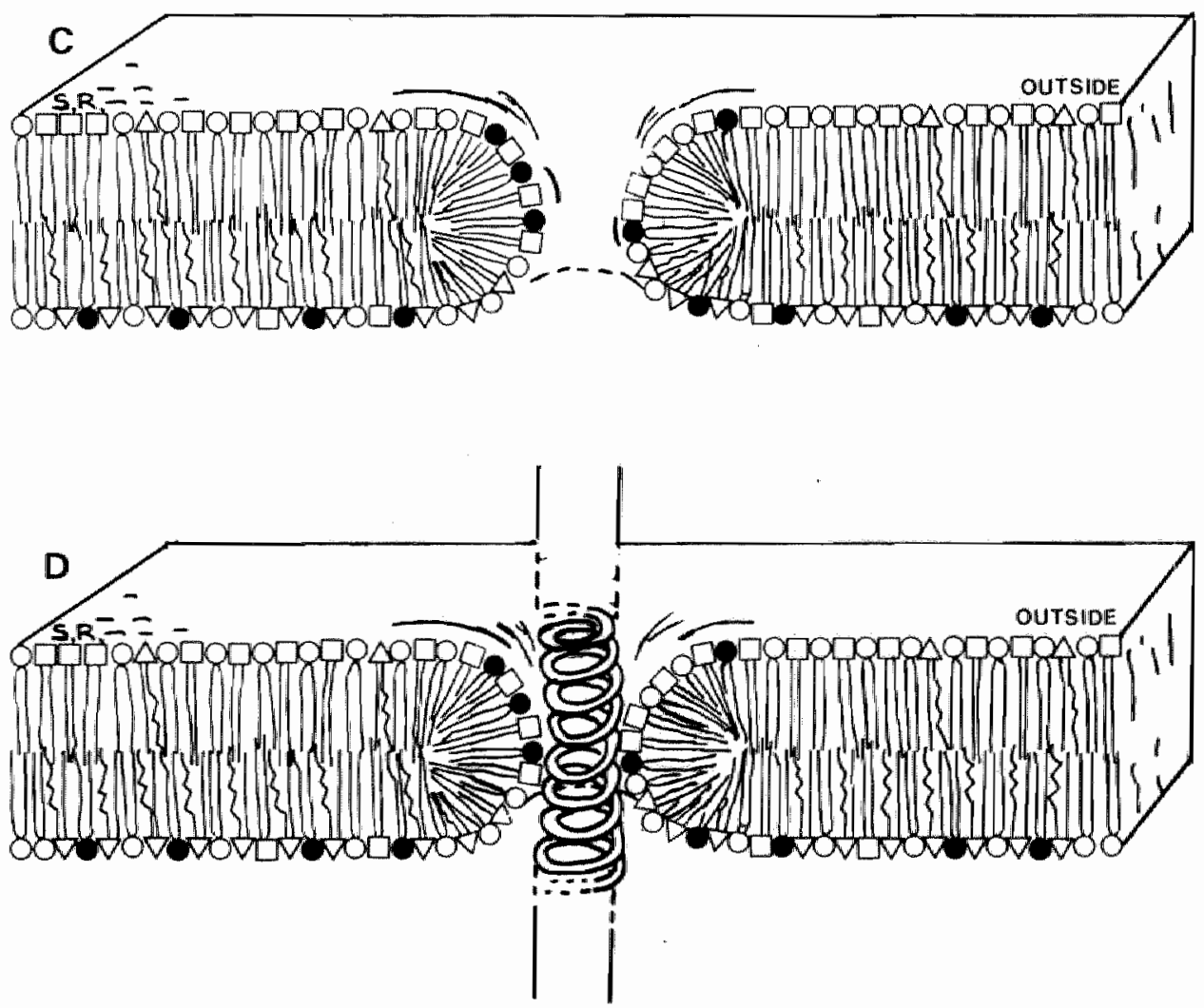
derived from findings presented in chapter 6. Generation of flipsites is related to stimulation of calpain, both kinetically and quantitatively. For reasons discussed in the first part of this chapter, involvement of calpain is taken to indicate involvement of membrane skeleton interactions in the generation of flipsites. In addition, flipsites in activated platelets allow bidirectional and aspecific transport of membrane lipids. This is concluded from the inward movement of sphingomyelin (SphM) which accompanies the outward movement of PS (chapter 1), and from the participation of TMA-DPH in rapid flipflop (chapter 6). Moreover, flipsites in activated platelets are transient, as indicated by the disappearance of rapid flipflop within 30 sec after stimulation by ionomycin (chapter 6).

Ultimately, transbilayer movement of lipids along flipsites will be limited by the speed at which these sites can be reached, i.e. by the lateral diffusion coefficient (D) of the lipid in question. In general, $D$ is about $10^{-7} \mathrm{~cm}^{2} / \mathrm{s}$ for phospholipids [549]. Assuming a globular form with a radius of $1 \mu \mathrm{m}$ for platelets, the platelet surface will be about $10^{-7} \mathrm{~cm}^{2}$. These data suggest that transbilayer lipid asymmetry over the platelet plasma membrane can be lost on a time scale of seconds, assuming the presence of flipsites, at which the activation energy for transbilayer movement is absent, i.e. at which both leaflets are interconnected. This limiting time scale is close to that of rapid flipflop in ionophore-activated platelets, suggesting that flipsites in activated platelets will have a pore structure which allows direct contact between the two leaflets.

Formation of hydrophilic pores in lipid bilayers is determined by the edge energy, which is directly related to the radius of curvature of the wall of the pore [549]. The energy barrier for pore formation can be overcome by high lateral pressures in the bilayer [549]. In view of these characteristics of pore formation in lipid bilayers, it is suggested that the difference in lateral pressure between the two leaflets of the platelet plasma membrane, as implicated by the fluidity gradient, will drive the formation of pores when the stabilization of the inner leaflet by cytoskeletal proteins is locally disabled by the action of calpain. This model can be extended to include the formation of non-bilayer structures close to the wall of the pore, thereby increasing the radius of curvature and thus reducing the energy for pore formation. Forma- tion of non-bilayer structures would involve cone-shaped lipids (chapter 1), like PE with unsaturated acyl chains, which is a major consittuent of the inner leaflet of the platelet plasma membrane [98, 494]. Formation of non-bilayer structures is in line with findings that the lipids of the inner leaflet of the erythrocyte plasma membrane do not form stable lipid bilayers [291], especially in the presence of $\mathrm{Ca}^{2+}$.

A pore model for flipsites in the plasma membrane of activated platelets, based on considerations mentioned above, is shown in Fig. 5. This model contains an annulus of hexagonal phase lipid, which may not be a prerequisite. A further addition to this model could be a transmembrane protein in the central core. The presence of such a protein can lower the energy for pore formation by supplying a hydrophilic transmembrane surface. Involvement of a transmembrane protein would allow regulation of pore formation by conformational changes resulting in exposure or disappearence of the hydrophilic transmembrane surface. In addition, membrane permeability may not be impaired by the presence of a transmembrane protein within the pore, as it most likely will be in the absence of such a protein. To account for the transient nature of flipsites in activated platelets, there must be mechanisms for relaxation of the membrane pore. In the protein-free pore, the local constraint of the fluidity gradient will be removed upon flipflop, and hence the driving force for pore formation is lost leading to closure of the pore. The protein containing pore may be closed by changes in the hydrophilic protein surface, lining the wall of the pore, as well. The pore model for rapid flipflop in activated platelets contains several features which can be tested. The amphipathic helixlike interaction between cytoskeletal proteins and the inner leaflet of the platelet plasma membrame can be demonstrated by appropiate phospholipid photoaffinity labels, or possibly in model systems with lipid monolayers. In addition amphipathic helices can be recognized directly from the primary structure of cytoskeletal proteins [176]. Finally, the occurrence of hydrophilic pores should lead to, at least temporarily, enhanced permeabilities for ions or non-electrolytes.

In conclusion, the kinetics of flipflop in activated platelets suggest transbilayer movement via pores in the plasma membrane. Transmembrane hydrophilic pores may be formed locally 
upon relief of the stabilizing interaction between cytoskeletal proteins and the plasma membrane through limited proteolysis by calpain. The driving force for pore formation is the difference in lateral tension between the two leaflets of the plasma membrane, which is related to the fluidity gradient. Formation of non-bilayer structures and the presence of a transmembrane protein in the pore may further facilitate pore formation. Flipflop itself will re- sult in pore relaxation by elimination of the driving force, the fluidity gradient.

\section{ACKNOWLEDGEMENT}

Sylvia Roomberg is gratefully acknowledged for the skillful drawings (Figs. 2, 4 and 5). 


\section{REFERENCES}

[1] ADACHI,Y, KOBAYASHI,N., MURACHI,T,, HATANAKA,M. (1986) $\mathrm{Ca}^{2+}$-dependent cysteine proteinase, calpains I and II are not phosphorylated in vivo. Biochem.Biophys.Res.Commun., 136: 1090-6.

[2] ADAMS,D.A., SMITH,W.B., POWELL,R.C. CARRAWAY,K.L. (1980) Electron spin resonance and fluorescence observations on erythrocytes, erythrocyte membranes, 13762 MAT-A ascites adenocarcinoma cellis, and their membranes, effects of membrane perturbations. Membr.Biochern, 3: 207-28.

[3] ADELSTEIN,R.S., POLLARD,T.D., KüHL,W.M. (1971) Isolation and characterization of myosin and two myosin fragments from buman blood platelets.

Proc.Natl_Acad Sci.USA, 68: 2703-7.

[4]ADNOT,S., POIRIER-DUPUIS,M., FRANKS,DJ., HAMET,P. (1982) Stimulation of rat platelet adenylate cyclase by an endogenous $\mathrm{Ca}^{2+}$-dependent proteaselike activity. J.Cyclic Nucleoride Res., 8. 103-18.

[5]ADUNYAH,S.E., DEAN,W.L. (1987) Regulation of buman platelet membrane $\mathrm{Ca}^{2+}$ transport by CAMPand calmodulin-dependent phosphorylation. Biochim.Biophys.Acta, 930: 401-9.

[6]ADUNYAH,S.E., DEAN,W.L. (1985) Inositol triphosphate-induced $\mathrm{Ca}^{2+}$ release from human platelet membranes. Biochem. Biophys.Res.Commust., 128: 12748 a.

[7]AHKONG,QF, BOTHAM,GM., WOODWARD,A.W., LUCY,J.A. (1980) $\mathrm{Ca}^{2+}$-activated thiolproteinase activity in the fusion of rat erythrocytes induced by benzyl alcoholl. Biochem J." 192: 829-36.

[8]AMBLER,J., BIRCH,J., MAGUIRE,E.D., WALLIS,R.B. (1985) Role of thromboxane A2.

Adv.Eup. Med Biol., 192: 293-308.

[9]AMELOOT,M., HENDRICKX,H. (1984) Effect of oriental order on the decay of the fluorescence anisotropy in membrame suspensions. Experimental verification on umilamellar vesicles and lipid/o-lactalbumin complexes. Biophys.J., 46: 525-39.

[10]ANDERSON,E.R., FOULIKSJ.G, GODIN,D.V. (1981) The effect of local anaest hetics and antiarrihythmic agents on the responses of tabbit platelete to ADP and thrombin. Thromb. Haemost., 45: 18-23.

[11]ANDO, $Y .$, IMAMURA,S., YAMAGATA, Y., KITAHARA,A., SAII,H ${ }_{-}$MURACHI,T, KANNAGI,R. (1987) Platelet factor XIII is activated by calpain.

Biocherm Biophys.Res.Commum, 144: 48490.
[12]ARAISO; $T$, SHINDO, $Y$, ARAI,T. NITTAJ., KIKUCHI, Y., KAKIUCHI, Y, KO YAMA, T. (1986)

Viscosity and order in erythrocyte mernbranes studied with nanosecond fluorometry. Biorheology, 23: 467-83.

[13]ARDLIE,N.G., BELL,L.K., MCGUINESS,J.A. (1987) Synergistic potentiation by epinephrine of collagen or thrombin-induced $\mathrm{Ca}^{2+}$ mobilization in human platelets. Thromb.Res." $46: 519-26$

[14]ARDLIE,N.G., GARRETT,J.J., BELL,L,K. (1986) Collagen increases cytoplasmic free $\mathrm{Ca}^{2+}$ in buman platelets. Thromb.Resi, 42:115-24.

[15]ARDUINI,A., CHEN,Z., STERN,A. (1986) Phenylhydrazine-induced changes in erythrocyte membrane surface lipid packing. Biochim. Biophys.Acta, 862: 65-71.

[16]ARDUINI,A., STERN,A. (1985) Spectrin degradathon in intact red blood cells by phenylhydrazine.

Biochems Phamacol, 34: 4283-9.

[17] ASANO,K., ASANO A (1988) Binding of cholesterol and inhibitory peptide derivatives with the fusogenic hydrophobic sequence of F-glycoprotein of HVJ (Sendai virus): possible implication in the fusion reaction. Biochemisty, 27: 1321-9.

[18] ASCH,A.S., BARNWELL,J., SILVERSTEIN,R.L., NACHMAN,R.L. (1987) Isolation of the thrombospondin membrane receptor. J.Clin.Invest., 79: 105461.

[19],ASCH,A.S, LEUNG,L.L., POLLEY,M.J., NACH. MAN,R.L. (1985) Platelet membrane topography: colocalization of thrombospondin and fibrinogen with the glycoprotein $\mathrm{II}_{\mathrm{b}}-\mathrm{III}_{\mathrm{a}}$ complex. Blood, 66: 926-34.

[20],AU,K.S. (1987) Activation of erythrocyte membrane $\mathrm{Ca}^{2+}$-ATPase by calpain. Biochim. Biophlys.Acta, 905: 273-8.

[21] BALDASSARE,JJ, BAKSHIAN,S, KNIPP,M.A., FISHER,G.J.(1985) Inhibition of fibringen receptor expression and serotonin release by leupeptin and antipain. J.Biol.Chem, 260: 10531-5.

[22]BALLOU,L.R., DEWITT,L.M., CHEUNG,W.Y. (1986) Substrate-specific forms of human platelet phospholipase A2. J.Biol.Chem, 261: 3107-11.

[23] BANIK,N.L., HOGAN,EL, WHETSTINE,LJ, BALENTINE,J.D. (1984) Changes in myelin and axonal proteins in $\mathrm{CaCl}$-induced myelopathy in rat spinal cord. Cent.Nerv.Syst, Trauma, $1: 131-7$.

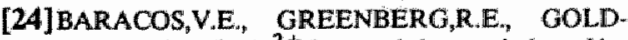
NERG,A.L. (1984) $\mathrm{Ca}^{2+}$ ions and the regulation of intracellular protein breakdown in muscle. in: "Ca ${ }^{2+}$

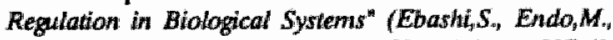
Imahori,K., Kakiuchi,S., Nishizuka,Y; eds.) pp. 227.42; Tokyo: Academic Press. 
[25] BARENHOLZ, Y, THOMPSON,T.E. (1980) Sphingomyelins in bilayers and biological membrane.s. Biachim. Biophys Acta, 604: 129-58.

[26]BARNES, MJ. (19811) Collagen polymorphism in refiation to the role of collagen-induced platelet aggre gation in haemost asis and thrombosis.

Prog Clin.Biol.Res., 54: 163-82

[27] BARUCH, $\mathrm{D}_{n}$, HEMKER,H,C, LINDHOUT, $\mathrm{T}$, (1986) Kinetics of thrombin-induced release and activathen of platelet fíactor V. EurJ.Biochem, 154:213-8.

[28] BEARER, EL, FRIEND, DS. (1986) (editorial:) Lipids of the platelet membrane. Lab.Inwest. 54: 119 21 .

[29]BEARER,E.L, FRIEND,D.S. (1982) An early membrane event in thrombin-stimulated platelets. J.Cell Biol, 95: 249a.

[30] BECKERLE,M.C, BURRIDGE,K, DEMARTINO,G.M., CROALL, D.E. (1987) Colocalization of $\mathrm{Ca}^{2+}$-dependent protease II and one of its substrates at siltes of cell adhesion. Cen, 51:569-77.

[31]BECKERLE,M,C, OHALLORAN,T, BURRIDGE, K (1986). Demonstration of a relationship between talin and P235, a major substrate of the $\mathrm{Ca}^{2+}$. dependent protease in platelets. J.Cell.Biochem., 30: 25070.

[32] BEECHEM J,M., BRAND,L. (1985) Time-resolved fluoreseence of proteins. Annu. Rev.Biochem., 54: 43-71.

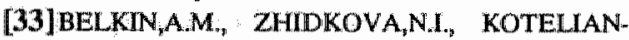
SKY,V.E. (1986) Localization of talin in skeletal and cardiac muscles. FEBS Lett, 200:32-6.

[34]BENGA, G., HOLMES, R.P. (1984) Interactions between components in biological membranes and their implications for membrane function. Prog Biophys.Mol.Biol, 43: 195-257.

[35] BENNET,JS. (1985) The platelet-fibrinogen reaction. in "Platelet membmane glycoproteins" (George, J.N., Nurden,A.T. Phillips,D.R.; eds.) pp. 193-214, New York: Plenum Press.

[36] BENINETT, V. (1985) The membrane skeleton of human erythrocytes and its implications for more complex cells. Ammu, Rev Biochem, 54: 273-304.

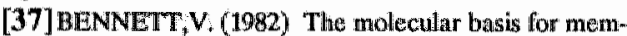
brane - cytoskelcton association in human erythrocytes. J.Cell.Biochem, 18, 49.65.

[38] BENTFELD-BARKER,M.E., BAINTON,D.F. (1982) Identification of primary lysosomes in human megakaryocytes and platelets. Blood, 59: 47281 .

[39]BERGMANN,W.L, DRESSLER, $V_{\text {, }}$ HAEST, C.W., DEUTICKE; B (1984) Cross-linking of SH-groups in the erythrocyte membrane enhances transbilayer reorientation of phospholipids. Evidence for a limited access of phospholipids to the reorientation sites: Biochün BiophysaAdcta, 769: 390-8.

[40]BERGMANN,WL,

DRESSLER, $\mathrm{V}$, HAEST, C.W., DEUTICKE, B. (1984) Reorientation rates and asymmetry of distribution of lysophospholipids between the inner and outer leaflet of the erythrocyte membrane, Biochin Biophys Acta, 772*328; 36 ,

[41] BERNDT,M,C, CAEN,JP. (1984) Platelet glycoproteins. Prog Memost. Thromb, it 1h-5a.
[42] BERNDT,M.C., DU, X.P., BOOTH,WJ. (1988) Ristocetin-dependent reconstitution of binding of von Willebrand factor to purified human platelet membrane glycoprotein $\mathrm{I}_{b} \mathrm{IX}$ complex. Biochemisty, 27: 633-40.

[43] BERNDT,MC, GREGORY,C., DOWDEN,G., CASTALDI,PA. (1986) Thrombin interactions platelet membirane proteins. Ann NY.ACadSCi, 485: $374-86$.

[44]BERNDT,MC, GREGORY,C, KABRAL,A, ZOLA, $H_{\text {, }}$ FOURNIER, D., CASTALDI,P.A. (1985)

Purification and preliminary characterization of the glycoprotein Ib complex in the human platelet membrane. Eur.J.Biochem., 151:637-49.

[45]BERNDT,M.C., PHILLIPS,D.R. (1981) Purification and preliminary physicochemical characterization of hurnan platelet membrane glycoprotein $\mathrm{V}$.

J.Biol. Chem, 256: 59-65.

[46] BERNDT,M.C., PHILLIPS,D.R. (1981) Platelet membrane proteins: composition and receptor function. Res.Monographs Cell Tissue Physiol, 5: 43-75 "Platelets in Biology and Pathology, wol. $2^{\text {th }}$ (Gordoni,J.L,; ed.) Elsevier/North Holland Biomedicat Press.

[47] BEST,L., JOHN,E, JHNIG, F. (1987) Order and fluidity of lipid membranes as determined by fluorescence anisotropy decay. Eur.Biophys. ${ }^{2}, 15: 87-102$

[48]BEVERS,E.M, COMFURIUS,P., NIEUWENHUIS,H.K., LEVY-TOLEDANO,S., ENOUF, J, BELLUCI,S, CAEN,J.P., ZWAAL,R.F.A. (1986) Platelet prothrombin converting activity in hereditary disorders off platellet function, BrJ.Haematol, 63: 335-45.

[49]BEVERS,E.M., COMFURIUS,P., VAN RIJN,J.L.M.L., HEMKER,H.C, ZWAAL,R.F.A. (1982) Generation of prothrombin-converting activity and the exposure of phosphatidylserine at the outer surface of platelets. EurJ.Biochem., 122: 429-36.

[50]BEVERS,E.M. ${ }_{n}$ COMFURIUS,P., ZWAAL,R.F. (1983) Changes in membrane phospholipid distribution during platelet activation. Biochim.Biophys.Acta, 736: $57-66$.

[51]BEVERS,EM, COMFURIUS,P., ZWAAL,R.F. (1982) The nature of the binding for prothrombinase at the platelet surface as revealed by lipolytic enzymes.

EurJ.Biochem, 122:81.5.

[52]BEVERS, EM.M, .. KARNIGUIAN,A., LEGRAND, Y I., ZWAAL, R.F. (1985) Collagen derived octapeptide inhibits platelet procoagulant activity induced by the combined action of collagen and thrombin. Thromb Res., 37: 365-70.

[53]BEVERS,EM, ROSING,J., ZWAAL,R.F.A. (1987) Platelets and coagulation. in "Platelets in Biology and Pathology, Vol.3" (MacIntyre,D.E., and Gordon, JLL," eds.) Res.Monogr.Cell Tissue Physiol, vol 13, pp. 127160, Elsevier Aftsterdam..

[54]BEVERS, E.M. ROSING $\mathrm{J}_{\mathrm{n}, \mathrm{q}}$ ZWAAL,R.F. (1985) Development of procoagulant binding sites on the platelet surface. Adv.Exp.Med.Biol, 192: 359-71.

[55] BHULLAR, R.P., HASLAM,R.J. (1987) Detection of $23-27 \mathrm{kDa}$ GTP-binding proteins in platelets and other cells. BiochemJ., 245:617-20.

[56]BIENZ,D, SCHINIPPERING,W., CLEMETSON ${ }_{v} K . J .(1986)$ Glycoprotein $V$ is not the thrombin activation receptor on human blood platelets. Blood, 
[57]BIGAX, $\}_{w,}$

DETERRE, $\mathbb{P}$.

PFISTER, $\mathrm{C}$., CHABRE,M. (1985) Fluoroaluminates activate transducin-GDP by mimicking the $\gamma$-phosphate of GTP in its binding site. FEBS Lett, 191: 181-5.

[58]BLLLGER,M., WALLIN,M., KARLSSON,JO. (1988) Proteolysis of tubulin and microtubule-associated proteins 1 and 2 by calpain I and II. Difference in sensitivity of assembled and disassembled microtubules. Cell Calcium, 9: 33-44.

[59] BITTMAN,R., CLEJAN,S. ROTTEM,S. (1983) Transbilayer distribution of sterols in mycoplasma membranes: a review. Yale J.Biol.Med., 56: 397-403.

[60]BLACHE, D, CLAVATTI,M, OJEDA,C. (1987) Platelet aggregation and endogenous 5-HT secretion in presence of $\mathrm{Ca}^{2+}, \mathrm{Sr} 2+$ and $\mathrm{Ba} 2+$. Effects of $\mathrm{Ca}^{2+}$ antagonists. Thromb.Res., 46: 779-9.1.

[61] BLIGH,E.G., DYER,W.J. (1959) A rapid method of total lipid extraction and purification.

CanJ. Biocherm. Physiol, 37: 911-7.

[62]VAN BLITTERSWIJ,W.J., VAN DER MEER,B.W., HILKMMANN,H. (1987) Ouantitative contributions of cholesterol and the individual classes of phospholipids and their degree of fatty acyl (un)saturation to membrane fluidity measured by fluorescence polarization. Biochemisty, 26:1746-56.

[63]BLOJ „B., ZILVERSMIT,D.B. (1982) Heterogeneity of Rabbit Intestine Brush Border Plasma Membrane Cholesterol. J.Biol.Chem., 257: 7608-14.

[64]BONNET, $D_{\ldots,}$ BEGARD,E (1984) Interaction of anilinonaphtyl labeled spectrin with fatty acids and phospholipids: a fluorescence study. Biochem.Biophys.Res.Commun, 120: 344-50.

[65]BOSIA,A, LOSCHE, W, PANNOCCHIA,A TREVES,S, GHIGO,D, TILL,U., PESCARMONA,G. (1988) Regulation of arachidonic acid-dependent $\mathrm{Ca}++$ influx in human platelets.

Thromb. Haemosh, 59: 86-92.

[66]BÖTTCHER,C.J.F., VAN GENT,CM., PRIES,C. (1961) A rapid and sensitive sub-micro phosphorus determination. Anal.Chim.Acta, 24:203-4.

[67]BOURGUIGNON,L.Y, WALKER,G., BOURGUIGNON,G.J. (1985) Phorbol ester-induced phosphorylation of a transmembrane glycoprotein (GP 180) in human blood platelets. J.Biol.Chem, 260: 11775-80.

[68]BOYLES, J, FOXJ.E, PHILLIPS,D.R., STENBERG,P.E. (1985) Organization of the cytoskeleton in resting discoid platelets: preservation of actin filaments by a modified fixation that prevents osmium damage.

I.Cell Bioh, 101: 1463-72.

[69]BRADFORD,H.N., ANNAMALAI,A., DOSHI,K,, COLMAN,R.W. (1988) Factor $V$ is activated and cleaved by platelet calpain: comparison with thrombin proteolysis. Blood, 71: 388-94.

[70]BRADY,R.C., CABRAL,FR., SCHIBLER,MJ., DEDMAN,J.R. (1985) Cellular localization of calmodulin and calmodulin-acceptor proteins. in: ${ }^{n} \mathrm{Ca}^{2+}$ and Cell Physiolog" (Mame, D; id) pp 140-47, Berlin: Springer Verlag

[71]BRANTON,D (1982) Membrane cytoskeletal interactions in the human erythrocyte. Cold Spring Harbor Symp.Quant.Biol, 46: 1-5.
[72] BRANTON,D, COHEN,C.M., TYLER,] (1981) Interaction of cytoskeletal proteins on the human erythrocyte membrame, Cell, 24:2432

[73]BRASS,LF. (1985) $\mathrm{Ca}^{2+}$ transport across the platelet plasma membrane. A role for membrane glycoproteins $\mathrm{I}_{\mathrm{b}}$ and $\mathrm{IL}_{\mathrm{a}}$.J.Biol. Chem, 260:2231-6.

[74]BRASS, L.F. (1984) $\mathrm{Ca}^{2 *}$ homeostasis in unstimulated platelets. J.Biol.Chem., 259:12563-70.

[75]BRASS,L.F, JOSEPH,SK. (1985) A role for inositol triphosphate in intracellular $\mathrm{Ca}^{2+}$ mobilitation and granule secretion in platelets. JiBiol.Chein., 260: 15172 9.

[76]BRASS,L.F., LAPOSATA,M., BANGA,A.S., RITTENHOUSE,S.E. (1986) Regulation of the phosphoinositide hydrolysis pathway in thrombin-stinulated platelets by a pertussis toxin-sensitive guanine nucleotide. binding protein. Evaluation of its contribution to platelet activation and comparisons with the adenylate cy. clase inhibitory protein, Gi. J.Biol. Cherm., 261: 16838-47.

[77]BRASS,L.F., SHATTIL,S.J. (1984) Identification and function of the high affinity binding sites for $\mathrm{Ca}^{2+}$ on the surface of platelets. J.Clin./nuest, 73: 626-32.

[78]BROEKMAN,M.J. (1986) Stimulated platelets release equivalent amounts of arachidonate from phos: phatidylcholine, phosphatidylethanolamine, and inositides. J.Lipid Res., 27: 88491.

[79]BROEKMAN,M.J., HANDIN.R.I., DERKSEN,A., COHEN,P. (1976) Distribution of phospholipids, latty acids, and platelet factor 3 activity among subcellular fractions of human platelets. Blood, 47:963-71.

[80] BRONNER,C, LANDRY, $Y_{\text {, }}$, FONTENEAU,P., KUHRY,J,G. (1986) A fluorescent hydrophobic probe used for monitoring the kinetics of exocytosis phenomena, Biochemistry, 25: 2149-54.

[81] BROWN,A.M., BIRNBAUMER,L. (1988) Direct G protein gating of ion chamnels. Am.J.Physiol., 254: H401-10.

[82]BRYAN, J, COLUCCIO,L.M. (1985) Kinetic analysis of F-actin depolymerization in the presence of platelet gelsolin and gelsolin-actin compilexes. J.Cell Biol., 103: 1236-44.

[83]BURGOYNE,R,D, CHEEK,T.R., OSULLIVAN, $A_{\text {JJ }}$. (1987) Receptor-activation of phosphtolipase-A $A_{2}$ in cellular signalling. Trends Biochem.Sci, 12 ." 332-3.

[84]BURN,P. (1988) Phosphatidylinositol cycle and uts possible involvement in the regulation of cytoskeletonmembrane interactions. J.Call. Biowhem., 36\% 15-24.

[85] BURN,P, ROTMAN,A, MEYER,R.K, BURGER,M.M. (1985) Diacylglycerol in large ea-actinin/actin complexes and in the gytoskeleton of activated platelets. Narure, $314: 469-72$.

[86]BURRIDGE,K, CONNELL,L. (1983) Talin: a cytoskeletal component concentrated in adhesion plaques and other sites of actin-membrane interaction. Cell Motil., 3: 405-17.

[87]BYGRAVE,F.L., REINHART,P.H ${ }_{\text {, }}$ TAY. LOR,W.M. (1985) Mitochondrial Ca ${ }^{2}$ " fluxes and their role in the regulation of intracellular $\mathrm{Ca}^{2+}$. in: "Ca ${ }^{2+}$ and Cell Physiology" (Marme, D.; ed.) pp 94-104, Berlin: Springer Verlag. 
[88]CAMPBELL, IM, PAW AGI, A, B. (1980) The ef: fect of polypeptide-Ipid interactions on the ultranolus spectrum of the oletinic bond of the lipid.

Can. Biochem, 58. 345-51.

[89]CARAFOLI, (1987) Intracdlular $\mathrm{C}^{2}$ " homeos Lasis. Anmu. Rev. Bochem, 50:395-433

[90]CARLSSON, MARKEY, F, BLIKSTAD, PERSSON,T, LNDDBERG,U. (1979) ReOMganization of actin in platclets stimulated thy thiombin as measured by the DNase In inhifition asssay.

Prow Nat Acad Sci.USA, 76. 6376-80.

[91]CARRELL,N.A., FITZGERALD,LA. WTEINER, $\mathrm{B}_{*}$ ERICKSON,H,P* PHLLIPS,D.R. (1985) Structure of human platelet membrane glycoproteins $\mathrm{I}_{\mathrm{b}}$ and $\mathrm{II}_{\mathrm{a}}$ as determined by electron microsopy. Jiol Chem, 260:17439.

[92]CASPAR,D L.D. KIRSCHNER,D.A. (1971)

Myclin membrane structure at 10 resolution. Natwe, 23: $46-52$

[93]CASTELLAMI, L, OFFER, G, ELLIOTT, A., $_{*}$ O'BRIEN,EJ. (1981) Structure of fllamin and the Factin-hesvy merohlamin complex

Rew. Coll.Motil, 2: 193-202.

[94]DE CHAFFOY DE COURCELLES,D. ROEVENS, $P_{\text {. }}$ VAN BELLE, H. DE CLERCK, (1987) The synergistic effect of serotonin and epinephrine on the human platelet at the lewel of signal trans duction. FEBS Leth, 279: 28378

[95]CHANDRA,R, JOSHI,P.C, BAJPAI, V.K, GUPTA, C,M. (1987) Menbrane phospholipid organization in $\mathrm{Ca}^{2+}$-loaded human erythrocytes. Bio chim. Biophys Acta, $902 ; 253-62$.

[96]CHAP,H., PERRET,B.; PLANTAVID,M., LACHAHCI,H, DOUSTE-BLAZY, (1987) Topography of platelet membrane phospholipids. in: "iplatelets in Biology and Pathology, III" (Machryre, D.E. Gardon,.L.; eds.) Res.Mowogr. Cell Tissue Physiol, 13: 191-204, Ansterdam: Elsewier.

[97] CHAP,H, SIMON, M.F, FAUVEL J $_{u y}$ PLANTAVID,M, MAUCO, O $_{n,}$ DOUSTE-BLAZY,L. (1985) Relationship between phospholipid metabolism and intracellular Cal 2 mobilization during platelet activation. Nown.Rew.Fr: Hewatol, 27: 220 33 .

$[98]$ CHAP, HI, ZWAAL, R.F WAN DEENEN, WL. (1977) Action of highily purified phospholipases on blood platelets. Evidence for an asymmetric distribution of phospholipids in the surface membrane. Biochim Biophy Acta, 467:14604.

[99]CHESTERMAN,C,N, BERNDT,M.C. (1986) Pltelet and wessel wall interaction and the genesis of atherosclerosis. Clin Haemärol, $15,323-53$.

[100]CHIANG, $\mathrm{T}$ M., KANG,A H (1982) Isolation and purification of collagen-) receptor from human platelet membrane. J.Biol Chem, 257: 7581-6.

[101]CHIGNARD,M, KERALY, GI NUNEZ, D, CO FFIER E, BENVENISTE, (1987) PAF-acether and platellets. in: Prateless in: Biology and Patholog, MT (Maclngre, D.E., Gondon,ilL; ads) Res.Monogr.Cell Tissue Physiol $13: 280.315$, Almsterdam. Elsewier.

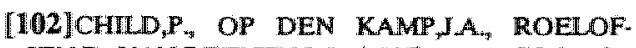
SEN,B, WAN DEENEN,LL. (1985) Molecular specties compposition of membrane photphatidylcholine inlluences the rate of cholesterol efflux from human ery. throcyues and wericles of erythrocyte lipid. Biochin Bio physw 4 icta, $814: 237-46$

[103]CHOE,H.R., SCHLEGEL,R,A, RUBTN,E, WL LIAMSON,P WESTERMAN, MP. (1986) Alteration of red cell membrane organization in sickle cell anaemia. Bir J.Hatmatol., 63: 761-73.

$[104]$ CHOE,HA, WILLIAMSON, $P_{*}, \quad$ RUBIN,E, SCHLEGEL, R. A. (1985) Disruption of phospholipid asymmetry in erythrocyte wesicles deficient in spectrim.

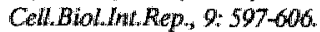

[105]CHRISTLANSSON,A., KUYPERS,FA., ROELOFSEN,B., WIRTZ,K.W., OP DEN KAMPJA. (1984) A comparative fluorescence polarization study of cis-parinaroy-phosphatidylcholine and diphenylhexatriene in membranes containing different amounts of cholesterol. Chem. Phys Liphds, 35: 247-58.

[106]CLARK,MR, MEL, $S_{*,}$ LUPU,F, FRIEND, D.S. (1987) Influence of the membrane undercoat on filipin perturbation of the red blood cell membrane. Exp.Cell Res: $171: 321-30$.

[107]CLASSEN,J., HAEST ${ }_{3}$ C.W., TOURNOIS,H, DEUTICKE,B. (1987) Gramicidin-induced enhancement of transbilayer reorientation of lipids in the erythrocyie membrane. Biochentisty, 26: 600412 .

[108]CLEMETSON,K. (1985) Glycoproteins of the platelet plasma membrane. in "Platelet membrane glyco proteins" (George, J.N., Nurden,A.T., Phillips, D.R,; eds.) Pp.51-85, New York: Plenum Press.

[109]CLEMETSON,KJ, LUESCHER,E.F. (1988)

Membrane glycoprotein abnormalities in pathological platelets. Biochim.Biophys.Acta, 947: 53-73.

[110]CLEMETSON,KJ, MCGREGOR,J I. (1987) Characterization of platelet membrane glycoproteins. in." Platelets in Biolony and Pathology, IIT" (MaclitDre, D.E., Gordon,J.Lo, eds.) Res.Monlogr. Cell Tissue Plugriol, 13: 1-36, Ambiterdanti: Elsivier.

[111] COCKROFT, S. (1987) Polyphosphoinosittide phosphodiesterase: regulation by a novel guanine nucleotide binding protein, $\mathrm{Gp}$. Trends Biochem Sci, 12: 75 \&

[112]COHEN,A.M., LIU,S.-C. DERICK, L.H. PALEK, (1986) Ultrastructural studies of the interaction of spectrin with phosphatidylserine liposomes. Blood, 68: $920 \%$

[113]COHEN,A.M. LIU,S.C., LAWLEIR, J, DE. RICK, L, PALEK, I. (1988) Identification of the protein 41 binding site to phosphatidylserine vesicles. Biochemistry, 27:6149:

[114]COHEN,C.M. (1983) The molecullar organization of the red cell membrane skeleton. Semin Fentolo, 20: $141-58$

[115]COHEN,L (1985) The mechanism of clot retraction. in "Platelet membrane givcoproteins" (Geome, J.N, Nurden,A.T, Phillips,D.R, eds.) pp.209-323, New York: Plenum Press.

$[116]$ COHEN, (1979) The contractile system of blood platelets and its function. Meth.Achiew.Exp.Pathol, 9 : 40-86. 
[117] COLLER,B.S. (1987) Blood elements at surfaces: platellets. Ann NY Aicad Sici, 516: 362-79.

[118]COLLER,B.S. (1986) Activation affects access to the platelet receptor for adhesive glycoprotteins. J.Cell Biot, 103: 451-6.

[119]COLLER,BS. (1982) Effects of tertiary amine local anesthetics on won Willebrand factor-dependent platelet function: alteration of membrane reactivity and degradation of GPI b by a $\mathrm{Ca}^{2+}$-dependent protease(s). Blood, 60: 7311-43.

[120]COLLER,B.S. (1985) Platelet-won Willebrand factor interactions. in: "Platelet membrane glycopmoteins" (Geonger.N., Nurdert,A.T., Phillips, D.R.; eds.) pp. 21544. New York: Plenum Press.

[120a]COLLER,B.S. (1985) A new murine monoclonal antibody reports an activation-dependent change in the conformation and/or microenvironment of the platelet glycoprotein $\mathrm{Il}_{\mathrm{b}} / \mathrm{IIH}_{\mathrm{a}}$ complex. J.Cin.Imwest., 76: 101-8.

[121 1 COLLER,B.S., PEERSCHKE,E,I., SCUDDER,L.E, SULLIVAN,C.A. (1983) Studies with a murine monoclional antibody that abolishes ristocetininduced binding of won Willebrand factor to platelets: additional evidence in support of GPIb as a platelet receptor for von Willebrand factor, Blood, 61: 99-110.

[122]COLLIER,N.C., WANG,K. (1982) Purification and properties of human platelet P235. A high nalecu. lar weight protein substrate of endogenous $\mathrm{Ca}^{2+}$-activated protease(s). J.Biol.Chem, 257: 6937-43.

[123] COLLIER,N.C, WANG,K. (1982) Human platelet P235: a high Mr protein which restricts the length of actin filaments. FEBS Leth, 143:205-10.

[124] COMFURTUS,P, BEVERS,EM., ZWAAL,R.F. (1985) The inwolwement of cytoskeleton in the regulation of transbillayer movement of phospholipids in human blood platelets. Biochint. Biophys-Alcta, 815: 1438.

[125]COMFURIUS,P., BEVERS,E.M., ZWAAL,R.F. (1983) Stimulation of prothrombinase activity of pllatelets and eryturocytes by sub-lytic treatment with phospholipase C from Clostridium welchii. Biochem.Bio phys. Res. Commum, II7: 803-8.

[126]COMFURIUS,P, ZWA.AL,R.F. (1977) The enzymatic synthesis of phosphatidylserine and purification by CM-cellulose column chronatography. Bio chim. Biophys Acta, 488:36-42.

[127]CONNOR,J., SCHROTT,AJ. (1988) Transbilayer movement of phosphatidylserine in erythrocytes: inhibition of tramsport and preferential labeling of a 31,000 dalton protein by sulfiydryl reactive reagents. Biochem istry, $27:$ 848-51.

[128]CONNOR,J, SCHROIT,A.J. (1987) Determina tion of lipid asymmetry in human red cells by resonance energy transfer. Biachemisty, 26: 5099-105.

[129]COOLICAN,S.A., HATHAWAY,D.R. (1984) Effect of L-osphatidylinositol on a vascular smooth muscle $\mathrm{Ca}^{2+}$-dependent protease. Reduction of the $\mathrm{Ca}^{2+}$ re quirement for autolysis. N.Biol.Chem, 259: 11627-30.

[130]COOLICAN,S.A, HAIECH,y, HATHAWAY,D.R. (1986) The role of subunit autolysis in actio vation of smooth muscle $\mathrm{Ca}^{2+}$-dependent proteases. J. BiollChem, 261: 41706 .
[131] OOOPER,R.A. LESLIEMA, FISCHKOFFS, SHINTTZKY,M, SHATTLL,S, J, (1978) Factors in fluencing the lipid compasition and fludity of red cell membranes in vitro: production of red cells possessing more than two cholesterals per phospholipid. Brochenistry, $17,327,3 \%$

[132]COTE,G, LEWIS,W.G., SMLLIE,LE. (1978)

Non-polymerizability of platelet tropomyosin and its NH2 and COOH-terminal sequences. FEBS Lat. $9 \%$ : $237-41$.

[133]COTTIN,P, AZANZA],L, VIDALENC,P, DU. CASTAING,A., VALIN, C, OUALI,A. (1981) Characterization and purification of a $\mathrm{Ca}^{2+}$ ion-activated neutral proteinase inhibitor in rabbit skeletal muscle $R e^{-}$ prod Nutr.Dev, $21: 309-17$.

[134]COTTIN,P., VIDALENC.P.L DUCASTAING A. (1981) $\mathrm{Ca}^{2+}$-dependent association between a $\mathrm{Ca}^{2+}$ actiwated meutral protentase (CaANP) and its specific inhibitor. FEBS Letr, 136:221 1-4.

[135] COX J A (1986) $\mathrm{Ca}^{2+}$-calmodulin interaction and cellullar function. J.Cardiovasc.Pharroacol, 8 (suppl 8). $\$ 48-5 \$ 1$.

[136]CRAIG,5.W., POLLARD,T.D. (1982) Actinbinding proteins. Trends Brochem.Sci, $7: 88.22$.

[137]CRANNEY,M., CUNDALL,R.B., JONES,G.R., RICHARDS,I.T., THOMAS,E.W. (1983) Fluorescence lifetime and quenching studies on some interesting diphenylhexatriene membrane probes. Biochim. Biophys.Acta, 735: 18-25.

[138]CRAWFORD,N. (1985) Structure and organisa* tion of platelet membranes . Adv.Exp.Med Biot, 192: I* 13.

[139]CRAWFORD,N., CASTLE, A.G. (1976) Tubulin and other microtubule associated proteins of the blood platelet. In: "Contractile systems in non-musche tis"sues. (Perry SV, et al., ed.), pp. 117-31. Amisterdam: Nonh-Hollaxd Publ.

[140]CROALL,D.E., MORROW, J, DEMARTINO,C.N, (1986) Limited proteolysis of the erythroeyt membrane skeleton by $\mathrm{Ca}^{2}{ }^{+}$dependent proteinnsos. Biochin. Biophyss Acta, 882: 287-96.

[141] CULLIS,P.R., DE KRUIJFF, B. (1979) Lipüd polymorphism and the functional roles of lipids in biologicat membranes. Biochim. Biophys Acha, 550: 300420 .

[142] CULLIS,P.R., VERKLEY,A.J (1979) Modulathon of membrane structure by $\mathrm{Ca}^{2+}$ and dibucaine as

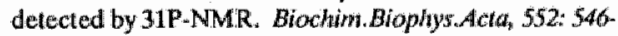
51 .

[143]DABROWSKA, $\mathrm{R}_{,}$GOCH, A., OSINSKA, $\mathrm{H}_{*}$ SZPACENKO,A., SOSINSK1, (1985) Duall effect of filamin on actomyosin ATPase activity. JMuscle Ress. Cell, Motill, 6: 29-42.

[144] DALEKE,D.L. HUESTIS,W.H. (1985) Incorporation and tramslocation of aminophospholipids in human erythrocytes. Biochenisty, 24: $5406-16$

[145] DANIEL,J.L, DANGELMAIER,CA, SMITH, J, B. (1987) Formation and meiabolism of inos:tol 1,4,5-trisphosphate in human platelets. Biochent., 246: 100214. 
[146]DA PRADA,M., RICHAARDS,IG, KETTLER,R. (1981) Amine storage organelles in platelets.

Res.Monographs Cell Tissue Phystiol, 5: 107-45. "Platelets in Blotogy and Pathology. wol.2" (Gondon, J.L; ed, Elsevier/Nonh-Holland.

[147] DA YIES, G.E. (1984) Association of actin with the platelet membrane. Biochim.Biophysicta, $72: 149-60$.

[148]DAVIES, GE, COFEN,CM. (1985) Platelets contain proteins immunologically related to red oell spectrin and protein 4.1. Blood, 65: 52-9.

[149]DAVIES,G.E, PALEK, J. (1982) Platelet protein organization: analysis by treatment with membrame-permeable cross-linking reagents. Blood; 59:502-13.

[150]DA VIES,P.J. WALLACH, D., WILLINGHAM,M., PASTAN,I, LEWTS,M.S. (1980) Self-association of chicken gizzard filamin and heavy merofilamin. Biochernisty, 19: 1366-72.

[151]DAVIES,P.J., WALLACH,D., WILLINGHAM,M.C. PASTAN, I, YAMAGUCHI,M ROBSON,R.M. (1978) Filamin-actin interaction. Dissociation of binding from gelation by $\mathrm{Ca}^{2+}$-activated proteolysis. J.Biol. Chem., 253: 4036-42.

[152] DAY,H.J., RAO,A.K. (1986) Evaluation of platelet function. Semin. Hematol, 23: 89-101.

[153]DEBUS,E, WEBER,K, OSBORN,M. (1981) The cytoskeleton of blood platelets wiewed by immunofluorescence microscopy. Eur.J.Cell Biol, 24:45-52.

[154]VAN DEENEN,L.L.M. (1981) Topology and dy. namics of phospholipids in membranes. FEBS Lett. 123" 3-15.

[155]DEINUM,G, VAN LANGEN,H., VAN GINKEL, G., LEVINE, Y.K. (1988) Molecular order and dynamics in planar lipid bilayers: effects of unsaturation and sterols. Biochemistry, 27; 852-60.

[156]DEMARTINO,G.N., BLUMENTHAL, D.K. (1982) Identification and partial purification of a factor that stimulates $\mathrm{Ca}^{2+}$-dependent proteases. Biochemistry, 21: 4297-303.

[157]DEMEL,R.A., GEURTS VAN KESSEL,W.S., ZWAAL, R.F. ROELOFSEN,B, VAN DEENEN, $L$, L. (1975) Relation between various phospholipase actions on human red cell membranes and the interfacial phospholipid pressure in monolayers.

Biochim Biophys.Acta, 406: $97 \cdot 107$

[158]DEMEL,R.A, JANSEN,J.W.C.M., VAN DUCK,P.W.M., VAN DEENEN,LL.M. (1977) The preferential interaction of cholesterol with different classes of phospholipids. Biochim.Biophys_Acta, $465: 1$ 10.

[159]DEMEL,R.A., DE KRUYFF,B. (1976) The function of sterols in membranes, Biochim.Biophys.Acta, 457: $100-32$

[160]DETWILER,T.C., MCGOWAN,E.B. (1985) Platelet receptors for thrombin. Adu.Ex.Med.Biol, 192 . 15-28.

[161] DEVAUX,P,F ${ }_{w}$ SEIGNEURET,M. (1985) Specificity of lipid-protein interactions as determined by spectroscopic techniques. Biochim.Biophys.Acta, 822 : 63-125.
[162]VAN DIEUEN ${ }_{p}, G_{,}$TANS, $G_{3}$, ROSING, J, HEM KER,H.C. (1981) The role of pliospholipid and factor $\mathrm{VII}_{\mathrm{d}}$ in the activation of bovine factor $\mathrm{X}$. J.Biol Chem, 256: 3433-42.

[163]VAN DUCK,P.W.M, VAN ZOELEN,EJJ., SELDENRUK, R, VAN DEENEN,L.L.M., DE GIERJ. (1976) Callorimetric behavior of individual phospholipid classes from human and bovine erythrocyte membranes. Chem.Phys. Lipids, 17: 336-343.

[164] DIMTTRO O,D.\$., JAIN,R.K. (1984) Membrane stability. Biochim. Biophys Acta, 779: 437-68.

[165]DINGUS,J., HWO,S., BRYAN,J. (1986) Identifcation by monoclonal antibodies and characterization of human platelet caldesmon. J.Cell Biol, 102: 1748-57.

[166]DONNER,M., STOLTZ,J.F. (1985) Comparative study on fluorescent probes distributed in human ery. throcytes and platelets. Biorheology, 22: 385-97.

[167] DOOLITTLE,R.F. (1984) Fibrinogen and fibrim. Arnut.ReN.Biochem. 53: 195-229.

[168] DRESSLER, V., HAEST,C.W., PLASA, G., DEU TICKE,B., ERUSALIMSKY,J.D. (1984) Stabilizing factors of phospholipid asymmetry in the erythrocyte membrame. Biochim. Biophys-Aicka, 775: 189-96.

[169]DRUMMOND,A.H., MACINTYRE,D.E (1987)

Platelet inositol lipid metabolism and $\mathrm{Ca}^{2+}$ flux. in. "Platelets in Biology and Patholog, IIJ" (MacIntyre, D.E. Gordon,J.L.; eds.) Res.Monogr.Cell Tissue Physiol., 13. 373-431, Amsterdam: Elsevier.

[170]DUFOURCQ, J, FAUCON,J.F., MAGETDANA,R., PILENI,M.P., HLNE,C. (1981) Peptidemembrane interactions. A fluorescence study of the binding of oligopeptides containing aromatic and basic residues to phospholipid wesicles. Biochim.Bio phys_Acta, 649:67-75.

[171] VAN DUIJN,G. LUIKEN,J, VERKLEU,AJ, DE KRUUFF,B. (1986) Relation between lipid polymorphism and transbillayer movement of lipids in rat liver microsomes. Biochim.Biophys_Acta, 863: 193-204.

[172]VAN DUUN,G., VALTERSSON, $\mathrm{C}_{\text {, }}$ CHOINACKI,T., VERKLEU,A.J., DALLNER,G., DE KRUIJFF, B. (1986) Dolichyl phosphate induces nonbillayer structures, vesicle fusion and transbilayer movement of lipids: a model membrane study. Biochim Biophys.Acta, 861: 211-23.

[173]DUPORTAIL, G, WEINREB,A. (1983) Photochemical changes of fluorescent probes in membranes and their effect on the observed fluorescence anisotropy values, Biochim.Biophys Acta, 736: 171-7.

[174]DU, $X$, BEUTLER,L., RUAN, $C$, CASTAL DI,P.A., BERNDT,M.C. (1987) Glycoprotein $I_{b}$ and glycoprotein IX are fully complexed in the intact platelet membrane. Blood, 69: 15247.

[175]EIRIN,M.T, CALVETE,JJ., GONZALEZRODRIGUEZJ. (1986) New isolation procedure and further biochemical characterization of glycoproteins $I_{b}$. and $\mathrm{III}_{\mathrm{a}}$ from human platelet plasma membrane.

Biochent $J_{i,}$ 240: 147-53.

[176]EISENBERG,D., WEISS,R.M., TERWIL LIGER,T.C. (1982) The helical hydrophobic moment a measure of the amphiphilicity of a helix. Nature, 299 . $371-4$. 
[177] EK, B., HELDIN,C.-H. (1986) Specific cleavage of the fibroblast receptor for platelet-derived growth factor by an endogenous $\mathrm{Ca}^{2+}$-dependent thiol provease. EurJubochem, 155: 40\%13:

[178] VAN ELDIK,LJ, WATTERSON,D.M. (1985) Callmodulin structure and function. in: ${ }^{\circ \mathrm{Ca}^{2+}}$ and Cell Physiology" (Mame, D. ed.) pp 105-26, Berlin: Springer Verlag.

[179]EMORI,Y, KAWASAKI,H, IMAJOH,S, KAWASHIMA,S, SUZUKI,K. (1986) Isolation and sequence analysis of CDNA clones for the small subunit of rabbit $\mathrm{Ca}^{2+}$-dependent protease. J.Biol Chern, $26 \%$ $9472-6$

[180] EMORI, Y, KAWASAKI,H, SUGIHARA, $H_{,}$, IMAJOH,S., KAWASHIMA, S, SUZUKI,K. (1986)

Isolation and sequence andyses of CDNA clones for the large subunits of two isozymes of rabbit $\mathrm{Ca}^{2+}$-dependent proterse. Jiol.Chem., 261: 9465-71.

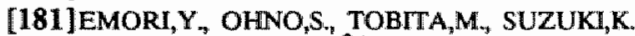
(1986) Giene structure of $\mathrm{Ca}^{2+}$-dependent protease re. tains the ancestral organization of the $\mathrm{Ca}^{2+}$-binding protein gene. FEBS Lett., 194: 249-52.

[182] ENGEL,L.W., PRENDERGAST,F.G. (1981)

Values for and significance of order parameters and "cone angles" of fluorophore rotation in lipid bilayers. Biochemistry, 20: 7338-45

[183]ENGLISH,D D $_{*}$ DEBONO,DJ, GABIG,T,G. (1987) Relationship of phosphatidylinositol bisphosphate hydrolysis to $\mathrm{Ca}^{2+}$ mobilization and functional activation in fluoride-treated neutrophils. J.Chin.Irwest., 80: 145-53.

[184] ENOMOTO,K.I., SATO,R. (1977) Asymmetric binding of cyochrome b5 to the membrane of human erythrocyte ghosts. Bioch im. Biophys Acta, 466: 136-47.

[185] ENOUF, $J_{\%}$ BREDOUX, $R$, BOURDEAU, $N_{\text {, }}$ LEVY-TOLEDANO,S. (1987) Two different Ca ${ }^{2+}$ transport systems are associated with plasma and intracellular human platelet membranes. J.Biol.Chem., 262 . 92937

[186]ENOUF, $]_{\text {, BREDOUX, }} \mathbf{R}_{\text {, }}$ BOIZARD, B. WAU TIER,JLL, CHAP,H, THOMAS,J, DE METZ,M, LEVY-TOLEDANO,S. (1984) Simultaneous isolation of two platelet membrane fractions: bibchemical, inmunological and functional characterization. Biochem.Biophys.Res.Comnun, 123: 50-8

[187] ENOUF, J, GRAUD,F,BREDOUX, R, BOURDEAU, $N_{*}$ LEVY-TOLEDANO $S_{n}$ (1987) Possible role of a CAMP-dependent phosphorylation in the $\mathrm{Ca}^{2+}$ rellease mediated by inositol $1,4,5$-trisphosphate in human platelet membrane wesicles. Biochim. Btophys.Acta, 928 . 76-82.

[188] ENYEDI,A, SARKADI,B, FOELDES-PAPP, $Z$, MONOSTORY,S, GARDOS,G. (1986) Demonstration of two distinct $\mathrm{Ca}^{2+}$ pumps in human platelet membrane vesicles. A.Biol Chem, 261: 9558-63.

[189]EFAND,RM. (1983) The amphipathic helix its possible role in the interaction of glucagon and other peptide hormones with membrane receptor sites.

Trends Biochem.Sci, 8: 205-7.
[190]EPAND,R.M, EPAND,R.F, ORLOWSK, R.C., SCHLUETER, R, BONI, L, T, HUI,SW. (1963) Amphipathic helix and its relationship to the interaction of calcitonin with phospholipids. Biochentising, 22.5074 84.

[191]ERNE; $P_{*}$ SCHACHTER,M FABBRO,D. MILES,C.M. SEVER,P.S. (1987) Cint tramsients in human platelets monitored by aequorim, fura 2 and quin-2: effects of protein kinase $\mathrm{C}$ activation and inhibition. Biochem.Bioptust Res. Commuth, $145,6672$.

[192] ETEMADI,A.-H. (1980) Membrane asymmetry A survey and critical appraisal of the methodology. If. Methods for assessing the unequal distribution of lipids. Biochim. Biophys.Acra, 604, 423-75.

[193]EVANS,T, BROWN,M.L, FRASER,E.D., NORTHUP,J.K (1986) Purification of the major GTP. binding proteins from placental nembranes.

J. Biol. Chem, 261: 7052-9.

[194]FARMER,I,B.T., H.ARMON FIELD,D.A. (1985) DR studies of the erythrocyte membrane skeletal protein nework: influence of the state of aggregation of spectrin on the physical state of membrane proteins, bilayer lipids, and cell surface carbohydrates. Biochim. Biophyss 4 ctia, $821: 420-30$

[195]FAUVEL,J., CH.AP,H., ROOUES, V., LEVY. TOLEDANO,S., DOUSTE-BLAZY,L (1986) Biochemical characterization of plasma membranes and intracellular membranes isolated from human platelets using Percoll gradients. Biochim Biophys Acta, 856 : 155-64.

[196] FEINSTEIN,M.B ${ }^{4}$ BECKER, E.L, FRASER,C. (1977) Thrombin, collagen and A33187 stimulated endogenous platelet arachidonate metabolism: differential inhibition by PGE1, local anesihetics and a serine-protease inhibitor. Prostaglandins; 14: 1075-93.

[197] FEINSTEIN,M.G, FIEKERS ${ }_{n}$, FRASER,C. (1976) An analysis of the mechanism of local anesthetic inhibition of platelet aggregation and secretion. J.Phar. macol Exp. Ther, 197: 215-28.

[198]FEINSTEIN "M.B., RODAN, G.A. CUTLLR,L.S. (1981) Cyclic AMP and $\mathrm{Ca}^{2+}$ in platelet function.

Ress.Monographs Cell Tissue Physiol., 5: 437-72. "Platelets in Biology and Pahology, nol. $2^{\text {n }}$ (Gordon,J.L.; ed.) Elsovier/Norih-Holland.

[199] FENTON,J.W, 2D (1981) Thrombin specificity. Ann.NYAcad Sci, 370: 468-95.

[200] FIDELIO,G.D. MAGGIO,B. CUMAR,F,A. (1982) Interaction of soluble and membrane proteins with monolayers of glycosphingolipids. Biochem. 203 : $717-25$.

[201] FISHER,K.A. (1976) Analysis of membrane halves: cholesterol. Proc Nan Alcad Si. USA, 73: 173-7.

[202] FITZGERALD,LAA., PHILLIPS, D.R. (1985) $\mathrm{Ca}^{2+}$ regulation of the platelet membrane glycoprotein $I_{b}-\mathrm{II}_{\mathrm{a}}$ complex J.Biol Chew, 260:11366-74.

[203]FITZGERALD,LA., PONCZ,M., STEINER,B., RALL,S.C . JR, BENNETT JS, PHILLIPS,D.R. (1987) Comparison of $\mathrm{CDNA}$-dierived protein secuences of the human fibronectin and vitronectin receptor a-subunits and platelet glycoprotein $\mathrm{II}_{6 .}$ Biochemistry, 26:8158-65. 
[204] FOX, E. B. (1987) The piatelet cytoskeleton. in

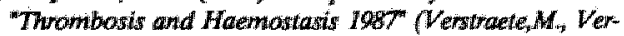

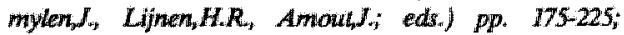
Leuven University Press, Lewven.

[205]FOX,ED, (1985) The organization of platelet conitractile proteins. in "Platelet membrane ghycoprom teins" (Creonge,I.N, Nunden,A.T. Phillips,D.R," eds.) pp.273-298, New York Plenum Press.

[206] FOX,J. (1985) Linkage of a membrane skeletion to integrall membrane glycoproteins in human platelets. Identification of one of the glycoproteins as glycoprotein l, N.Chin Invest, $76: 1673-89$.

[207] FOX,J (1985) Identification of actin-binding protein as the protein linking the membrane skeleton to gycoproteins on platelet plasma miembranes.

J.Biol.Chem, 260:119707.7.

[208]FOXJE. (1985) Hydrolysis of cytosikeletal proteins by the $\mathrm{Ca}^{2+}$-dependent protease during platelet activation. Adv. Exp.Med.Biol, 192" 201-13.

[209]FOX,IE, AGGERBECK,LP., BERNDT,M.C. (1988) Structure of the glycoprotein $I_{b} . \mathbf{I X}$ complex from platelet membranes. J.Biol Chem, 263: 41882-90.

[210] FOX, ال, E, BOYLES,J.K., BERNDT,M.C., STEF FEN,P.K, ANDERSON,L.K. (1988) Identification of a membrame skeleton in platelets. ${ }_{1}$ Cell Biol., 106.1525 38.

[211]FOX,JE, BOYLES,J.K, REYNOLDS,C.C., PHILLIPS,D R, (1984) Actin filament content and organization in unstimulated plateletsi J.Cell Biol, $98:$ 1985.91 .

[212]FOX,J.E, GOLL,D.E , REYNOLDS,C.C., PHIL LIPS, D.R. (1985) Identification of two proteins (actinbinding protein and P235) that are hydrolyzed by endogenous $\mathrm{Ca}^{2+}$-dependent protease during platelet aggregation. J.Biol.Chem, 260:1060-6.

[213] FOX,J.E, PHILLIPS,D.R. (1982) Role of phosphorylation in mediating the association of myosin with the cytoskeletal struckures of human platelets.

J.Blol.Chem, 257: 4120-6.

[214] FOX,J,E B, PHILLIPS,D,R. (1983) Polymerization and organization of actin filaments within platelets. Semin Hematol, $20: 243-60$.

[215]FOX, $\mathrm{E}_{\text {" }}$ REYNOLDS,C,C, MORROW,J.S. PHILLLIPS,D.R. (1987) Spectrin is associated with membrane-bound actin fillaments in platelets and is bydrolyzed by the $\mathrm{Ca}^{2+}$-dependent protease during platelet activation. Bloow, 69: 537-45.

[216] FOX,J,E, REYNOLDS,C.C, PHILLIPS,D.R. (1983) $\mathrm{Ca}^{2+}$-dependent proteolysis occurs during platelet aggiregation. J.Biol, Chem., 258; 9973-81.

[217]FRANCK,P.F, BEVERS,EM, LUBN,B.H. COMFURIUS,P., CHIU,D.T, OP DEN KAMP,JA., ZWAAL,R.F., VAN DEENEN,L.L., ROELOFSEN,B. (1985) Uncoupling of the membrane skeleton from the lipid bilayer. "The cause of accelerated phospholipid fip-nop leading to an enhanced procoagulant activity of sickled cells. J. Cin.Jinvest., 75:183-90.

[218] FRANCK,P.F, CHIU,D,T, OP DEN KAMP,JA., LUBIN,B, WAN DEENEN,L,L, ROELOFSEN,B. (1983) Accelerated transbilayer movement of phosphatidylcholine in sickled erythrocytes. A reversible process. J.Biol Chem., 258: 8436-42.
[219] FRANCK,P.F, OP DEN KAMP,,$A$, LUEIN,B, BERENDSEN, W., JOOSTEN,P., BRIET,E, VAN DEENEN,L.L, ROELOFSEN,B. (1985) Abnormal transbilayer molbitity of phosphatidycholine in hereditary pyropoikilocytosis reflects the increased heat sensi-

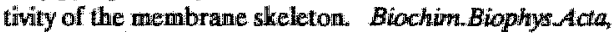
815: 259-67.

[220] FRANCK,P.F, ROELOFSEN,B, OP DEN KAMP, J (1982) Complete exchange of phosphatidylicholine from intact erythrocytes after protein crosslinking Blochim Biophys Alcto, 687:105-8.

[221] FUJTMURA, K, PHILLIPS,D,R. (1983) Ca $\mathrm{Ca}^{2+}$ cation regulation of gycoprotein $\mathrm{HL}_{\mathrm{b}}-\mathrm{IH}_{\mathrm{a}}$ complex for mation in platelet plasma membranes. JBioLChem. $258 \cdot 10247-52$.

[222]FULFORD,A.J.C, PEEL,W.E (1980) Lateral pressures in biomembranes estimated from the dynamics of fluorescent probes. Biochim Biophys Acto, 598 . $237-46$.

[223]FUSE,I, TAI,H.H. (1987) Stimulations of arachdonate release and inositol-1,4,5-triphosphate formation are mediated by distinct $\mathrm{G}$-proteins in human platelets. Biochem.Biophys.Res.Cammun, 146:659-65.

[224] GALLA,H.J,, LUISETTI,J. (1980) Lateral and transversal diffusion and phase tramsitions in erythrocyte membranes. An excimer fluorescence study. Biochim.Biophyrifata, 596: 10817.

[225]GANGULY,P, GOULD,N.L. (1979) Thrombin receptors of human platelets: thrombin binding and antithrombin properties of glycoprotein 1. BrJ.Haema10l., 42: $137-45$.

[226]GARDNER, J.M, HYNES,R.O. (1985) Interaction of fibronectin with its receptor on platelets. Cell, 42: 439.48 .

[227] GATES,R,E, KING,L.E.,JR (1985) Different forms of the epidermal growth factor receptor kinase have different autophosphorylation sites. Biochemistry, 24: $5209-15$.

[228] GEIGER,B. (1985) Microfilament-membrane interaction. Trends Biochem.Sci., 10, 456-61.

[229] GEIGER,B. (1983) Membrane-cytoskeleton interaction. Biochim. Biophys Acta, 737: 305-4\%.

[230]GEORGEJ], NURDEN,A.T., PHILLPS,D.R. (eds.) (1985) Platelet membrane proteins, New Yonk: Plenum Press.

[231] GERRARD, J,M , CARROLL,R.C. ISRAELS,S.J, BEATTIE,L.L. (1987) Protein phospliorylation. in: Platelets in Brology and Patholiogy, $I I T^{m}$ (MacImpre,D.E. Gordon,J.L* eds.) Res.Monogr.Cell Tissue Physiol, 13:317-351, Amsterdam: Elsevier.

[232] GERRARD,J.M. PETERSON,D.A., WHITE,J.G. (1981) $\mathrm{Ca}^{2+}$ mobilization. Res.Monographs Cell Tissue Physiol, 5. 407-36; "Platelets in Biology and Pathology. Vol.2." (Gordon,J.L., edi), Elsevier/Nonth Hollond.

[233]GERRITSEN,WJ., HENRICKS,P.A., DE KRUIJFF, B., VAN DEENEN,L.L. (1980) The transbilayer movement of phosphatidylcholine in vesicles reconstituted with intrinsic proteins from the human ery. throcyte membrane. Biochüm.Biophys Acta, 600: 607-19. 
[234]GERRITSEN,WJ, DE KRUUIFF,B, VERKIEU,AJ., DE GIER, J, WAN DEENEN,LL.L. (1980) $\mathrm{Ca}^{2+}$-induced isotropic motion and phosphatidylcholine flip-flop in phosphatidylcholine-cardiolipin bilayers. Biochim.Biophys-Acta, 598: 554-60.

[235] GILMAN,A.G. (1987) G-proteins: transducers of receptor-generatted signals. Annu.Revibiochem., 56 6.15-49.

[236]GILMAN_A.G. (1984) G-proteins and dual control of adenylare cyclase. Cell, 36:577-9.

[237]GLLMORE,R., COHN,N., GLASER,M. (1979)

Fluidity of LM cell membranes with modified lipid compositions as determined with 1,6-diphenyl-1,3,5-hexatriene. Biochemistry, 18: 1042-1049.

[238]GINSBERG,M.H., LOFTUS,J.C., PLOW,E.F. (1988) Cytoadhesins, integrins, and platelets.

Thromb. Haemast, 59: 1-6.

[239] GIOMETTI,C.S, ANDERSON,N.G. (1984) Prom tein changes in activated human platelets. Clin.Chem, 30: $2078-83$.

[240] GLASS,F., LIPPTON,H, KADOWITZ,P.J. (1980) Differential effects of local anesthetics and propranolol on arachidonic acid and adenosine 5'-diphosphate-induced aggregation in rabbit platelets. Prostaglandins Med, 5: 85-92.

[241]GLASER, T., KOSOWER,N.S. (1986) Calpaimcalpastatin and fusion. Fusibility of enythrocytes is determined by a protease-protease inhibitor [calpaincalpastatin] balance. FEBS Lett., 206: 115-20,

[242] GLASER, T., KOSOWER,N.S. (1986) Fusion of rat erythrocytes by membrane-mobility agent $\mathrm{A}_{2} \mathrm{C}$ depends on membrane proteolysis by a cytoplasmic cal. pain. EurJ.Biochem., 159: 387-92.

[243]GOGSTAD,G.O., KRUTNES,M.B., HETLAND,O, SOLUM,N.O. (1983) Comparison of protein and lipid composition of the human platelet $\alpha$-granule membranes and glycerol lysis membranes. Biochims.Biophys_Acta, 732: 519-30.

[244] GOMPERTS,B.D. (1983) Involvement of guanine nucleotide-binding protein in the gating of $\mathrm{Ca}^{2}$ by receptors. Nature, 306: 646.

[245] GOODMAN,S.R., SHIFFER,K. (1983) The spectrin membrane skeleton of normal and abnormal human erythrocytes: a review. AmL.Fhysiol, 244: CI2I-4I.

[246]GORDON,J.L. (1985) Endothelium as a modulator of platelet reactivity. Adv.Exp.Med.Biol., 192: 41925.

[247] GORDON,J.L., PEARSON,J.D. (1987) Biology of the vascullar endothelium. in: "Haemostasis and Thrombosis" (Bloom,A.L., Thomas,D.P,; eds.) pp. 303-311, Churchill Livingstone, Edimbungh.

[248]GRASSO,M., MORELLI,A., DE FLORA,A. (1986) $\mathrm{Ca}^{2+}$-induced alterations in the levels and subcellular distribution of proteolytic enzymes in human red blood cells. Biochem.Biophys.Res.Commutun "138: $87-94$.

[249]GRATZER,W.B. (1981) The red cell membrane and its cytoskeleton. Biochem J, 198: I-\&

[250] GIR YNKIEWICZ,G., POENIE,M., TSIEN,R.Y. (1985) A new generation of $\mathrm{Ca}^{2+}$ indicators with greatby improved thorescence properties. J.Biol.Chem, 200: 344050 .
[251]GULLICK; WI, DOWNWARD, J WATER * FIELD, M.D. (1985) Antibodies to the autophosphorylation sites of the epidermal growth factor receptor protein-tyrosine kinase as probes of structure and function. EMBO I, 4: 2869-77.

[252]GUY,G:R, GORDON,J, WALIKER,L, MICHELL,R.H., BROWN,G. (1986) Redistribution of protein kinase $C$ during mitogentsis of human $B$ lywnphocytes. Biachem.Bioplyys. Res. Commtuni. 135: 146-53.

[253] HACK,N., CRAWFORD,N (1984) Two-dimensional polyacrylamide-gel electrophoresis of the proteins and glycoproteins of purified human platelet surface and intracellular membranes. Biochem. $J_{\text {, }} 222: 235$ 46.

[254] HACK, N., CROSET,M., CRAWFORD,N (1986)

Studies on the bivalent-cation-activated ATPase activities of highly purified human platelet surface and in iracellular membranes. Biochem. $J, 233$ 661-8.

[255] HAEBERLE,J.R., COOLICAN,S.A., EVAN,A, HATHAWAY D.R. (1985) The elfects of a $\mathrm{Ca}^{2+} \mathrm{de}$ pendent protease on the ultrastructure and contractile mechanics of skinned uterine smooth muscle. J.Muscle Res.Cell.Motil, 6: 347-63.

[256] HAEST,C.W.M. (1982) Interactions between membrane-skeleton proteins and the intrinsic domain of the erythrocyte membrane. Biochim.Biophys Acta, 694 : 331-52.

[257]HAEST,C.W., DEUTICKE,B. (1976) Possible relationship between membrane proteins and phospholipid asymmetry in the human erythrocyte membrane. Biochin. Biophys Acta, 436: 353-65.

[258]HAEST, C.W, ERUSALIMSKY, J, DRESSLER, V., KUNZE, L, DEUTICKE,B. (1983) Transbilayer mobility of phospholipids in the erythrocyte membrane. Influence of the membrane skeleton.

Biomed.Biochim_Acta, 42: S17-21.

[259]HAEST,C.W., PLASA,G, KAMP,D., DEUTICKE, B. (1978) Spectrin as a stabilizer of the phospholipid asymmetry ith the human erythrocyte membrane. Biochim. Biophys. Acta, $509: 21-32$.

[260]HAGEN,I, BROSSTAD,F, GOGSTAD,G.O., KORSMO,R., SOLUM,N.O. (1982) Further studies on the interaction between thrombin and GP $\mathrm{l}_{b}$ using crossed immunoelectrophoresis. Effect of thrombin inhibitors. Thromb. Res., 27: 549-54.

[261] HALE,J.E, SCHROEDER,F. (1982) Asymmetric transbilayer distribution of sterol across plasma membranes determined by fluorescence quenching of dehy droergosterol. Eur.J.Biochem, 122: 649-61.

[262] HALL, T.G., BENNET, V. (1987) Regulatory domains of erythrocyte ankyrin. J.Biol.Chens., 262: 10537. 45.

[263] HALLAM,T.J., RINK, TJ. (1987) Insights into platelet function gaimed with fluorescent $\mathrm{Ca}^{2+}$ indicators. in: "Platelets in Biology and Pathology, III" (Maclntyre, D.E., Gordon,J.L., eds.) Res.Monogr. Cell Tissue Physiol, 13: 353-372, Amsterdam: Elsevier.

[264] HALLAM,T.J., RINK, T.J. (1985) Agonists stimulate divatent cation channels in the plasma membrane of human platelets. FEBS Leith, 186: 175-9. 
[265] HALLAM,TJ., SIMPSON,A,W, O'CONNOR,N, RINK, Ty. (1985) Control and interrellation of aggregattion and secretion; the roles of $\mathrm{Ca}^{2+}$, diacylghycerol and thromboxane wilh particular reference to ADP stimullation. Adv.Eup.Med.Biol, 192: 145-62

[265a]HANASAKI,K, NAKANO, T., ARIT A,H, (1987) Two phasic generation of thromboxiane $A_{2}$ by the action of collagen on rat platelets. Thromb. Ress, $46: 425-36$.

[266]HARDISTY,R.M. (1985) Molecular mechamism of platelet adhesion. Adv.Exp.Med. Biol, J92:411-8.

[267] HARMON,JT, JAMIESON, G.A. (1988) Platelet activation by thrombin in the absence of the high-affinity thrombin receptor. Biochemistry, 27: 2151-7.

[268]HARMON,J.T., JAMESON,G.A. (1986) The glycocalicin portion of platelet glycoprotein $\mathrm{I}_{\mathrm{b}}$ expresses both high and moderate affinity receptor sites for thrombin. A soluble radioreceptor assay for the interaction of thrombin with platelets. J.Biol.Chem, 261 : 132249.

[269]HARMON,J.T, J.AMIESON,G.A. (1986) Actiwation of pllatelets by $\alpha$-thrombin is a receptor-mediated event. D-phenylalanyl-L-prolyl-L-arginine chloromethyl ketone-thrombin, but not $\mathrm{N} \alpha$-tosyl-L-lysine chloromethyl ketone-thrombin, binds to the high affinity thrombin receptor. J.Biol.Chem, 261: 15928-33.

[270] HARMON,J.T, JAMIESON,G.A. (1986) Platelet activation by $a$-thrombin is a receptor-mediated event. Ann NY,AcadSCL, 485: 387-95.

[271] HARRIS,H. (1981) Regulation of motile activity in platelets. Res.Monographs Cell Tissue Physiol., 5: 473500 "Platelets in Biology and Pathology, wol. 2" (Gom don, J.L L ; ed.) Elsevier/North Holland Biomedical Press.

[272]HASLAM,R.J. (1987) Signal transduction in platelet activation. in: "Thrombosis and Hemostasis 1987" (Nerstraete, M., Vermylen,J., Lijnen, $R$, Amoust,yl; edis.) pp. 147-174; Leiven University Press, Lewven.

[273]HASLAM,R.J., DAVIDSON,M.M. (1984) Potentiation by thrombin of the secretion of serotonin from permeabilized platelets equilibrated with $\mathrm{Ca}^{2+}$ bulfers. Relationship to protein phosphorylation and diacylglycerol formation. BiochemJ $J_{2} 222: 351-61$.

[274] HAASL_AM,R.J., LYNHAM,J.A. (1978) Relationship between phosphorylation of blood platelet proteins and secretion of platelet granule constituents II. Effects of different inhibitors. Tiromb.Res, 12.619-28.

[275] HATA YAMA,K. KAMBA YASH, J. KAWASAKKI, $T_{*}$ MORIMOTO, $K$, OHSHIRO, $T_{\text {, }}$, MORI, $T$. (1986) Interrelationship between secretion, protein phosphorylation and intracellular $\mathrm{Ca}^{2+}$ concentration in platelets stimulated by thrombin or thromboxane $\mathrm{A}_{2}$ analogue. Thromb.Rer, 4:761-70.

[276]HATHAWAY,D.R.,

WERTH,D.K.

HAEBERLE, J.R. (1982) Limited aut olysis reduces the $\mathrm{Ca}^{2+}$ requirement of a smooth muscle $\mathrm{Ca}^{2+}$-activated protease. J.Biol.Chem., 257: $9072-7$.

[277] HATTORI,A, TAKAHASHI,K (1982) $\mathrm{Ca}^{2+}{ }^{2}$-induced weakening of skeletal muscle Z-disks.

J.Biochem, 92: 387-90.
[278] HAVERSTICK,D.M., COWAN,J.F YAMADA, K.M., SANTORO,S.A. (1985) Inhibition of platelet adhesion to fibronectin, fibrinogen, and won Willebrand factor substrates by a synthetic tetrapeptide derived from the cell-binding domain of fibronectin. Blood, 66: 940-52.

[279]HAWIGER,J. (1987) Macromolecules that link phatelets following vessel wall injury. Ann. NY.Acad.Sci. 509: 131-41.

[280]HERMAN,B., ROE,MW, HARRIS, C., WRAY,B., CLEMMONS,D. (1987) Platelet-derwed growth factor-induced alterations in vinculin distribution in porcine vascular smooth muscle cells. Cell Motil.Cytoskeleton, 8: 9I-105.

[281] HER RMANN,A. MULLLER,P. (1986) A model for the asymmetric lipid distribution in the human erym throcyte membrane. BiosciRep, 6: 185-91.

[282] HESCHELER, J.,

ROSENTHAL, W: TRAUTWEIN,W, SCHULTZ, . $_{\text {. }}(1987)$ The GTPbinding protein, $\mathrm{Go}$, regulates neuronal $\mathrm{Ca}^{2+}$ channels. Nauure, 325: 445-47.

[283] HINCKE,M.T., TOLNAI,S. (1986) Phosphorylation of bovine cardiac $\mathrm{Ca}^{2+}$-activated meutral protease by protein kinase-C. Biochem.Biophys. Res. Commun., 137: 559-65.

[284] WA.N HOEK,A., VISSER,A.J.W.G. (1985) Artefact and distortion sources in time-correlated single photon counting. Anal.Instmum, 14: 359-78

[285] VAN HOEK,A., VER VOORT, J, VISSER,A.J.W.G. (1983) A subnanosecond resolving spectrofluorimeter for analysis of protein fluorescence kinetics. J.Biochem. Biophys. Methods, 7: 243-54.

[286]VAN HOEK,A, VOS,K, VISSER,A.J.W.G. (1987) Ultrasensitive time-resolved polarized fluorescence as a tool in biology and medicine. IEEE J.Quantum Electr, QE-23: 1812-20.

[287] HOFFMANN,W., PINK,D.A., RESTALL, C., CHAPMAN,D., (1981) Intrinsic molecules in fluid phospholipid bilayers. Flworescence probe studies. EurJ.Biochem., 14:585-9.

[288] HOLMSEN,H. (1985) Platelet metabolism and activation. Semin.Hematol, 22: 219-40.

[289] HOLMSEN,H., NILSEN,A.O., RONGVED,S. (1985) Energy requirements for stimulus-response coupling, Adv Exp.Med.Biol., 192:215-33.

[290] HOLMSEN,H., WEISS,HJ. (1979) Secretable storage pools in platelets. Assu. Rev.Med., 30:119-134.

[291] HOPE, M.J., CULLIS,P.R. (1979) The bilayer stability of inner monolayer lipids from the human erythrocyte. FEBS Lett. 107: 323-6.

[292]HORWITZ,A., DUGGAN,K, BUCK,C, BECKERLE, M.C. BURRIDGE, K. (1986) Interaction of plasma membrane fibronectin receptor with talin. A transmembrane linkage. Noture, 320:53\%-3.

[293] HOUSLAY,M.D. (1987) Egg activation unscrambles a potential role for IP4. Trends Biochem.Sil, 12:1. 2

[294] HOUSLAY,M.D. (1987) Ion channels controlled by guanine nucleotide regulatory proteins. Trends Biochern.SCi., 12: 167-68. 
[295]MAAJOH,S, KAWASAKI,H., SUZUKI, K (1986)

"The amino-terminal bydrophobic tegion of the small subunit of $\mathrm{Ca}^{2+}$-activated neutral protease (CANP) is essential for its activation by phosphatidylinositol.

J. Bhochem, $99: 12814$

[296]INOMATA,M., HAYASHI,M., NAKAMU. RA,M. IMAHORI, K, KAWASHIMA, S. (1985) Hy" drolytic and autolytic behavior of two forms of $\mathrm{Ca}^{2+}-\mathrm{ac}$ tivated neutral protease (CANP). NBiochem, 98: 40716.

[297]ISHI,H, CONNOLLY,T.M, BROSS,T.E, MAJERUS,P.W. (1986) Inositol cyclic triphosphate [inositoll 1,2-(cyclic) -4,5-triphosphate) is formed upon thrombin stimulation of thuman platelets.

Proc.Natl AcadSci.USA, 83: 6397-401.

[298]ISRAELACHVILIJ.N, MTTCHELL,D.J, NIN, HAM,B.W. (1977) Theory of self-assembly of lipid bilayers and vesicles. Biochim. Biophys.Acta, 470*185-201.

[299]JACKSON, R.L, PATTUS,F, DEMEL,R.A. (1979) Interaction of plasma lipoproteins with lipid monolayers. Biochim.Biophys_tcta, 5.56:369-87.

[299a] JAM, M.K., ZAKTM,D. (1987) The spontaneous incorporation of proteins into preformed billayers. Biochim. Biophysictata, 906: 33-68.

[300]JANDROT-PERRUS,M, GULLIN,M.C., NURDEN,A.T. (1987) Human y-thrombin: lack of correlation between a platelet functional response and glycoprotein $\mathrm{V}$ hydrolysis. Thromb.Haemost., 58 915 20

[301]JENNINGS,L.K, FOX,J.E, EDWARDS,H.H, PHILLIPS,D.R. (1981) Changes in the cytoskeletal structure of human platelets following thrombin activation. J.Blol. Chem, 256: 6927-32

[302] JOHNSTON,G.I, HEPTINSTALL,S. (1988) Identüty of saturable $\mathrm{Ca}^{2+}$-binding sites on blood platelets and their involvement in platelet aggregation.

Thmamb.Haemost., 59: 546l.

[303] JY,W , HA YNES, D. H. (1987) Thrombin-induced $\mathrm{Ca}^{2+}$ movements un platelet activation. Biochim.Bio phys Acta, 929: 88-102.

[304] JY,W WAYNES,D.H. (1984) Intracellular $\mathrm{Ca}^{2+}$ storage and release in the human platelet. Chlorotetracycline as a continuous monitor. Circ.Res., 55: 595-608.

[305]KAIBUCH, $K_{,}$, SANO $_{4} K_{n, *}$ HOSHIJMA, M., TAKAI, Y, NISHIZUKA.Y. (1982) Phosphatidylinositol turnover in platelet actiwation; $\mathrm{Ca}^{2+}$ mobilization and provein phosphorylation. Cell Colcium, 3: 323-35.

[306] KAIIWARA, $Y_{\text {, }}$ TSUJINAKA, $\mathbb{T}^{+}$SAKON,M., KAMBAYASHI, OHSHIRO,T, MURACHI, $T$, MORI,T. (1987) Elucidation of callpain dependent phosphorylation of myosin light chain in human platelets. Biochem.Int, 15: 935-44.

[307] KAMBAY,ASHI,I., KAJTWARA,Y, SAKON,M. OHSHIRO,T., MORI,T. (1986) Possible participation of calpain in myosin light chain phosphorylation of human platelets. Biochem.Int, 13:571-8.

[308] KAPLAN,KL. (1981) Platelet granule proteins: localization and secretion. Res.Monographs Cell Tissue Physiol, 5. 77-90 "Platelets in Bialogy and Pathology, woL2" (Goindon, ML, ed) Elsevier/Worth Holland Biomedical Press.

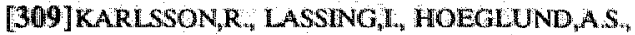

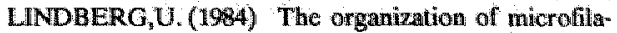
ments in spreading platelets: a comparison with fibroblastis and glial cells, JCell Physiol, 121:96-113.

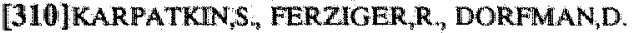
(1986) Crassed immuncelectrophoresis of human pla telet membranes. Effect of charge on associtation and dissociation of the glyooprotein CPIIb-GPIL membrane complex. JBiol Chem, 261" "14266-72

[311] KAUPP,U.B, KOCH, K.,W. (1986) Mechanisan of photoreception in vertebrate wision.

Biochemsis, 11: 41-7.

Thends

[312]KAWASAKI H, MMAJOH,S, KAWASHIMA,S, HAYASHL,H, SUZUKI,K. (1986), The small subumits of $\mathrm{Ca}^{2+}$ dependent proteases with different $\mathrm{Ca}^{2+}$ sensitivities are identical. J.Bucherth, 99: $7525-32$

[313]KAWATO, $S_{x_{y}}$ KINOSITA, JR, K, IKECAMI,A. (1978) Effect of cholesterol on the molecular motion in the hydrocarbon region of lecithin bilayers studied by anasecond fluorescence techniques: Biochentistry, 17 ; 5026-3\%.

[314] ]KEHREL,B. B,ALLEISEN, L. KOKOTT,R. MESTERS,R., STENZINGER,W. CLEMET" SON,KJ., VAN DE LOO,J. (1988) Defliciency of intact thrombospondin and membrane glycoprotein la in platelets with defective collagen-induced aggregation and spontaneous loss of disorder. Bloud, 71: 10748.

[315]KELUSKY,E.C.

BOULANGER, $Y_{\text {, }}$ SCHREIER, S., SMITH,I.C.P. (1986) A 2HI-NMR study on the interaction of the local anesthetic tetracaine with membranes containing phosphatidydserine. Biochivm.Biophys Alcta, 856: 85-90.

[316] KENNEY,D.M., CHAO,FC. (1980) Ionophoreinduced disassembly of blood platelet nicrotubules: ef: fect of cyclic AMP and indomethacin. J.Cell Plysiol., J03: 28908.

[317]KIENAST,J., ARNOUT, J, PFLIEGLER, $\mathrm{J}_{*, \text {, }}$ DECKMYN,H., HOET, B, VERMYLEN,J. (1987) Sodiumfluoride mimics effects of both agonists and antagonists on intact human platelets by simullaneous modulation of phospholipase $C$ and adcoylate cyclase activity. Blood, $69: 859-60$.

[318]KIER,A.B. SWEET,W.D., COWLEN,M S. $_{*}$ SCHROEDER,F. (1986) Regulation of transbilayer distribution of a fluoresecent sterol in tumot cell plasma

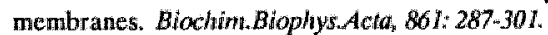

[319] KINLOUGH-RATHBONE, RL L, MUSTARD,J.F. (1987) Endogenous mediators of platielet

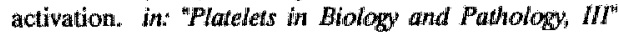
(MacIntyre,D.E, Gordon, JL, eds.j) Res.Monown.Cell Tissue Physiol., 13, 239-67, Amsterdich: Elsevier.

[320] KINOSITA,K,JR, KAWATO, S, IKEGAMI,A. (1984) Dynamic structure of biological and model membranes: analysis by optical anisotropy decay measurement. Adk biophys, $17: 147-203$,

[321] KINOSITA,JR,K, KAWATO,S, IKKEGAMI,A., YOSHIDA,S , ORII,Y. (1981) The effect of cytochrome oxidase on lipid chain dynamics. A nanosecond fluorescence depollarization study.

Biochim.Bio phys $A$ Acta, 647:7-17.

[322]KINOSITA,K,JR, KAWATO,S, IKEGAMI,A. (1977) A theory of fluorescence polarization decay in 
[323] KISHMOTO,A, KAJIKAWA,N, SHIOTA,M., NISHIZUKA, $Y$. (1983) Protedlytic activation of $\mathrm{Ca}^{2+}$ activated, phospholipid-dependent protein kinase by $\mathrm{Ca}^{2+}$ dependenit neutral protease. J.Biol Chem, 258 : $1156-64$.

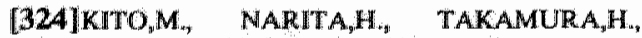
PARK, H, MA MATSUURA, T, TANAKA, K, (1986)

Dissociation of $\mathrm{Ca}^{2+}$ mobilization from brealkdown of phosphatididylinositol 4,5-bisphosphate in activated huunian platelets. J.Biochem, 99: 1277-80.

[325] KL _ALSNER,R D, KILEINFELD,A.M. (1984)

Lipid domains in membranes: in: "Cell Surfoce Dynamics. Concepts and Models." Receptors and Ligands in Intercellutar Communication. 3. 23-58 (Perelson,A.S., DeLisi, C., Wiegel,F.W; eds.), New York: Mareel Dekker Incs

[326]KLOCZEWIAK,M, TTMMONS,S., LUKAS,TJ, HAWIOER, I (1984) Platelet receptor recognition site on human fibrinogen. Synthesis and structure-function relationship of peptides corresponding to the carboxyterminal segment of the y-chain Biochemistry, 23: 176774.

[327] K.NIGGT,D.E., SCRUTTON,M.C. (1984) Cyclic nucleotidles controll a system which regulates $\mathrm{Ca}^{2+} \operatorname{sen}$ sitivity of platelet secretion. Nature, 309: 66-8.

[328] K.NUPP,CL., WHITE, G.C.,2D (1985) Effect of active site-modified thrombin on the hydrolysis of platelet-associated glycoprotein $\mathrm{V}$ by native thrombin. Blood, 65: 578-83.

[329] KKNUPP,C.L., WHITE, G.C, 2D (1984) The effect of platelet inhibitors on glycoprotein $V$ hydrolysis by thrombin. Thronb.Res., 34: 225-31.

[330] KOBAYASHI, T, OKAMOTO,H, YAMADA,J.I., SETAKA, M., KWAN,T (1984) Vesiculation of plateler plasma membranes. Dilauroyiglycerophosphocholine-induced shedding of a platelet plasma membrane fraction enriched in acetylcholinesterase activity Biochim.Biophys.Acta, 778: 210-18.

[331] KOMETANI,M., SATO,T., FUJI,T, (1986) Effect of membrane-interacting amphiphiles on association of membrame glycoproteins with assembled cytoskeletal proteins in concanavalin A-activated rabbit plam telets. Thromb.Res., 42:567-77.

[332] KORN,E.D. (1982) Actin polymerization and its regulation by proteins from nonmuscle cells. Physiol.Rev, 62: 672-737.

[333] KOSAKI, G., TSUINAKA,T, KAMBAYASHIJ., MORIMOTO, $K_{\text {i, }}$ YAMAMOTO, $K$, YAMAGAMI,K., SOBUE, K, KAKIUCHI,S. (1983) Specific cleavage of calmodulin-binding proteins by low $\mathrm{Ca}^{2}$-requiring form of $\mathrm{Ca}^{2+}$ activated neutral protease in himan platelets. Biochem.Int., 6: 767.75.

[334] KOTITE; N J, CUNNINGHAM;L.W. (1986) Specific adsorption of a platelet membrane glycoprotein by human insoluble collagen. J. Biol Chem, 261: 8342-7.

[335]KOTITE,NJ., STAROS,J.V., CUNNINGHAM L W. (1984) Interaction of specific platelet menbrane proteins with collagen: evidence from chemical cross-linking. Biochemistry, 23: 3099-104.

[336]KOWALSKA,M.A., CIERNIEWSKI,C.S. (1983)

Microenwironnent changes of human blood platelet membranes associated with fibrinogen binding.
[337] KRAMER,R.M., CHECANI,G.C., DEYKIN,A., PRITZKER, C.R, DEYKIN,D. (1986) Solubilization and properties of $\mathrm{Ca}^{2+}$-dependent human platelet phospholipase A2. Biochim.Biophys.Acta, 878: 394-403.

[338] KREES, J J.R. (1982) The topology of phospholitpids in artificial and biological membranes. J.Bicenerg.Biomembr, 14: 141-57.

[339]DE KRUIJFF, B., BAKEN,P. (1978) Rapid transbilayer movement of phospholipids induced by an asymmetrical perturbation of the bilayer. Biochim.Bio physiActa, 507: 38-47.

[340]DE KRUIIFF, B, CULLIS,P.R. (1980) The influence of poly-L-lysine on phospholipid polymorphism. Evidence that electrostatic polypeptide-phospholipid interactions can modulate bilayer/non-bilayer transitions. Biochim. Biophyss-Acta, 601: 235-40.

[341]DE KRUUFF,B., CULLIS,P.R., VERKLEIJ,AJI. (1980) Non-bilayer lipid structures in model and biological membranes. Trends Biochem.Sci, 5: 79-81.

[342]DE KRULJFF,B., VERKLEU,AJ., VAN ECHTELD,CJ.A, GERRITSEN,WJ., MOMBERS,C., NOORDAM,P.C. DE GIER,J. (1979) The occurrence of lipidic particles in lipid bilayers as seen by 31P NMR and freeze fracture electron microscopy. Biochim.Bio phys.Acta, 555: 200-9.

[343]DE KRUIJFF,B, WIRTZ,K.W. (1977) Induction of a relatively fast transbilayer movement of phosphatidylcholine in vesicles. A 13CNMR study. Biochim.Bio phys_Acta, 468: 318-26.

[344]DE KRUIJFF,B., VAN ZOELEN,EJJ. (1978) Effect of the phase transition on the transbilayer movement of dimyristoyl phosphatidylcholine in unilamellar wesicles. Biochim.Biophys.Acta, 511: 105-15.

[345]KUBINA,M., LANZA,F, CAZENAVE,J.P., LAUSTRIAT,G., KUHRY,J.G. (1987) Parallel investigation of exocytosis kinetics and membrane fluidity changes in human platelets with the fluorescent probe, trimethylammonio-diphenylhexatriene. Biochim.Biophys Acra, $901: 138-46$.

[346] KUBOKI,M., ISHII,H., KAZAMA,M. (1987)

Procalpain is activated on the plasma membrane and the calpain acts on the membrane. Biochim.Bio phys-Acta, 929: 16472.

[347] KUHRY,J.G., DUPORTAIL, $G$, BRONNER,C, LAUSTRIAT,G. (1985) Plasma membrane fluidity measurements on wholle living cells by fluorescence anjsotropy of trimethylammoniumdiphenythexatriene. Bio chim. Biophys_Acta, 845:60-7.

[348] KUHRY $Y_{0} J_{1}-G_{.,}$FONTENEAU,P., DUPORTAlL, G., MAECHLING, C., LAUSTRIAT,G. (1983)

TMA-DPH $i$ a suitable fluorescence polarization probe for specific plasma membrane fluidity studies in intact living cells. Cell Biophys., 5: $129-40$

[349] KUMAR,A, GUPTA,C.M. (1983) Red cell membrane abnormalities in chronic nyeloid leukaemia. $\mathrm{Na}$ ture, 303: 632-3.

[350] KUNICKI, T.J. (1985) Organization of glycoproteins within the platelet plasma membrane. in "Platelet membrane glycoproteins" (Geome,J.N., Nurden,A.T., Phillips, D. $R_{\text {; }}$ eds.) Pp.87-103, New York: Plenum Press.

[351] KUNICKI,TJ, MOSESSON,M.W, PIDARD,D. (1984) Cleavage of fibrinogen by human platelet $\mathrm{Ca}^{2+}$. 
[352] KUNICKI,T $J$, MONTGOMERY,RR, SCHULLEK, (1985) Cleavage human won Willebrand factor by platelet $\mathrm{Ca}^{2+}$-activated protease. Blood, 65: 3526.

[353]KUNICKI,TJ, NUGENT,DI., STAATS,SI, ORCHEKOWSKI,R.P., WAYNER,EA., CARTER, W.G. (1988) The human fibroblast class II extracellular matrix receptor mediates platelet adhesion to collagen and is identical to the platelet glycoprotein $\mathrm{I}_{\mathrm{a}}$ IIL complex. J.Biol.Chem., 263: 4516-9.

[354]KUYPERS,F.A., VAN LINDE-SIBENIUS TRIP,M, ROELOFSEN,B, OP DEN KAMP,JA, TANNER,M J, ANSTEE,D.J. (1985) The phospholipid organisation in the membranes of McLeod and Leach phenotype erythrocytes. FEBS Lett., 184: 204.

[355] LAD,P.M., OLSON,C. V., GREWAL, I.S., SCOTT,SJ. (1985) A pertussis toxin-sensitive GTPbinding protein in the haman neutrophil regulates mulliple receptiors, $\mathrm{Ca}^{2+}$ mobilization, and lectin-induced capping. Proc.Natl.Acad Sci.USA, 82: 8643-7.

[356] LAEMMLI,U.K. (1970) Cleavage of structural proteins during the assembly of the head of the bacteriophage 'T4. Narure, 227: 680-5.

[357]LAGARDE,M. (1987) Roles of cyclooxygenase and lipoxygenase metabolites in platelets. in: "Platelets in Biology and Pathology, MT" (Maclntyre,D.E., Gor don,J.L; eds.) Res.Monogr.Cell Tissuc Physiol, 13" 26988. Amsterdam: Elsevier.

[358] LAGARDE,M, GUICHARDANT,M., MENASHI,S, CRAWFORD,N. (1982) The phospholipid and fatty acid composition of human platelet surface and intracellular membranes isolated by high voltage free flow electrophoresis, J.Biol.Chem., 257: 31001-4.

[359] LAHAV,J. (1987) Identification of a surface protein of the rabbit blood platelet with high affinity for collagen. Exp. Cell Res., 168: 447-56.

[360] LAHAV, J, MEYER,F.A. (1981) On the rolle of the major platelet membrane glycoproteins in platelet adhesion to collagen. Thromb. Res., 22: 457-662.

[361] LAKOWICZ,J.R., CHEREK, $H_{2}$, BALTER,A. (1981) Phase fluorimetry with diphenylhexatriene: methodological aspects. J.Biochen.Biophys.Methods, 5; 134-46.

[362] LAM,S.C. PLOW,E.F. SMITH,M.A:, AN. DRIEUX,A., RYCKWAERT,J.J., MARGUERIE,G., GINSBERG,M.H. (1987) Evidence that arginyl-glycylaspartate peptides and fibrinogen $\gamma$ chain peptides share a common binding site on platelets. J.Biol.Chem, 262: 947-50.

[363] LANDON,F., GACHE, Y., TOUITOU,H., OLO. MUCKI,A. (1985) Properties of two isoforms of human blood platelet $\alpha$-actinin. EurJ.Biochem., 153: 231-7.

[364] LANGEN,VAN,H., LEVINE, Y.K, AMELOOT,M., POTTEL,H. (1987) Ambiguities in the Interpretation of Time-Resolved Fluorescence Anisotropy Maesurements on Lipid Vesicle Systems.

Chem.Phys.Leth, 140:304-400.

[365] LANGE, Y. (1984) The dynamics of erythrocyte membrame cholesterol. Prog.Clin.Biol. Ress, 159: 137-51.

[366] LAPETINA,E.G. (1987) The role of GTP-binding proteins in receptor activation of phospholipase $C$. Adu.Exp.Med Biol, 221: 95-100.
[367]LAPETINA,EG. (1983) Metabolism of inositides and the activation of platelets. Life $3 \mathrm{ch}, 32: 2069-82$

[368] LARSEN,N.E, SIMONS,E.R. (1981) Preparation and application of a photoreactive thrombin analogue: binding to human platelets. Biochemisiry, 20: $1141-7$.

[369] LASSING,I, LINDBBERG, U. (1988) Evidence that the phosphatidylinositol cycle is linked to cell motility. Exp. Cell Res, 174: 1-15.

[370]LASSING,I, LINDBERG, U. (1985) Speeifie interaction between phosphatidylinositol 4,5-bisphosphato and profilactin, Nature, $314: 472-4$.

[370a]LAU,S.H, VALE,J.R.W., KAISER,E.T, KZDY,FJ. (1983) Surface properties of an amphiphilic peptide hormone and of its analogi corticotropin-releasing factor and sauvagine. Proc.NathAcadiSciUS4 40 : 7070-4.

[371]LAWLER,J. (1986). The stractural and functional properties of thrombospondin. Blood, 67: 1197 200.

[372] LAZARIDES,E (1985) Assernbly of the plasma membrane of red blood cells in erythroidal development. Proceedings of the 13th Intentational Congress of Biochemistry, Amsterdam, Nethertands, 25-30 august 1985; abstruct MO-C19, page 19.

[373]LEGRAND, Y.J., KARNIGUIAN,A , FAUVEL,F, GUTMAN NN. (1983) The molecular interaction between platelet and wascular wall. Blood Cells, 9: 263-74.

[374]LEONARD II.P., SALPETER,M.M. (1982) $\mathrm{Ca}^{2+}$. mediated myopathy at neuromuscular junctions of normal and dystrophic muscle. Exp.Neurol, 76:121-38.

[375]LEREA,K.M., LIVINGSTON,J.N. (1986) A thiolsensitive degradative process of liwer uncouples autophosphorylation of the insulin receptor from insulin binding. Biochem.J., 236:535-42.

[376]LEVY-TOLEDANO,S., ENOUF, $\mathrm{J}_{,}^{*}$ BREDOUX,R., DE METZ,M, LEBRET,M. (1985) Mechanism of regulation of $\mathrm{Ca}^{2+}$ concentration by platelet membranes. Nowv Rev.Fr.Hematol, 27: 249-53.

[377] LEVY-TOLEDANO,S. MACLOUF, BRYON,P., SAVARIAU ${ }_{n} E_{\text {; }}$ HARDISTY,R.M., CAEN,J.P. (1982) Human platelet activation in the absence of aggregations: a $\mathrm{Ca}^{2+}$-dependent phenonicnon independent of thromboxane formation. Blsod, 59 : 1078-85.

[378]LEWIS,J.C, (1984) Cytoskcteton in platelet function. Cell.Muscle Motil, 5. 341-77,

[379]LEWIS, JC., WHITE,M.S., PRATER,T, PORTER,K.R., STEELE,RJ. (1986) Cyloskeletal changes during adhesion and release: a comparison of human and nonhuman primate platelets. Scon.Electron.Microsc., (PII): 199-208.

[380]LEWIS,J.C, WHITE,M.S., PRATER,T, PORTER,K.R., STEELE,RJ. (1983) Three-dimensional organization of the platelet cytoskeleton during adhesion in vitro: observations on human and monhuman primate cells. Cell Motil., 3: 589-6088.

[381] LIMBIRD,L.E. (1981) Activation and atteruation of adenylate cyclase. The role of GTP-bunding proteins as macromolecular messengers in receptor-cyclasc: coupling. Biachem-1., 195: 1-13. 


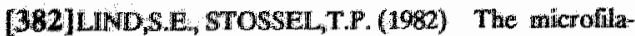
nent network of the platelet. Prog Hemost Thromb. 6: 6984.

[383]LIND,SE, YTN,H.L., STOSSEL,T.P. (1982)

Human platelets coratain gelsolin. A regulator of actun filament length. IC Climinvest, 69: 13847 .

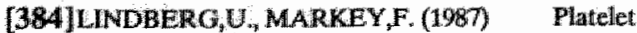
milicrofiaments and motility. in: "Plateleds in Biology and

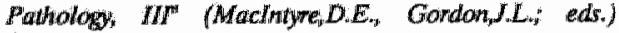
ResiMonowCell Tussue Physiol, 13: 205-237, Amsterdam: Elsevies.

[365]DE USLE R.C. WLLIAMS J,A. (1966) Regula tion of nembrane fusion in secretory excytosis AnumiRew Physiol, 48: 22.5-38.

[386]LOCHRIE,M.A, SIMON,MI. (1988) G-protein multiplicity in eakaryotic signal transduction systems. Biachemisty, $27: 4957-65$

[387]LOFTUS,I.C., ALBRECHT,R.M. (1984) Redistribution of the fibrinogen receptor of human platelets affer surface activation. 1. Cell Biol, 99: 822-9.

[388]LOFTUS,IC, CHOATE, ALBRECHT,RM. (1984) Platelet activation and cytoskeletal reorganizafion: wigh volkage electron microscopic examination of intact and Triton-extracted whole mounts. J.Cell Biot., 98. $2019 \mathrm{~m} 25$.

[389] LORAND,L, BJERRUM,OJ, HAWKINS,M. LOWE-KRENTZ,L., SIEFRING, G.E.,JR (1983) Degradation of transmembrane proteins in $\mathrm{Ca}^{2+}$ enriched human erythrocyes. An immunochemical study. J.Biol.Chem, 258:5300-5.

[390]LOW,M.G, CARROLL,R.C., COX,A.C. (1986) Characterization of multiple forms of phosphoinositidespecific phospholipase $\mathrm{C}$ purified from human platelets. BiochenJ., 237: 13945.

[391] LuSCHER,E.F. (1985) Plasma membrane receptors and platelet response. in "Plotelet membrane goyco proteins" (George,J.N., Murden, A.T, Phillips, D.R.; edts.) pp.3-9, Now Yonk: Plemum Press.

[392]LUX,S.E (1979) Dissecting the red cell membrane skeleton, Nature, $281: 426 \%$.

[393]LYNCH,G, BUDRY,M. (1987) Brain spetrin, calpain and long-term changes in synaptic efficacy.

Broin Res. Bull, 18. 809-15.

[394]LYNCH, G, BAUDRY,M (1984) The biochemistry of memory a new and specific hypothesis. Setence. 224: 1057-63.

[395] MACINTYRE, D.E

BUSHFIELD, M. SHAW A.M (1085) Regulation of platellet cytosolic free $\mathrm{Ca}^{2+}$ by cyclic nucleotides and protein kinase $\mathrm{C}$. FEBS Lat, $188: 383-8$

[395A] MACUNTYRE, D.E. POLLOCK, W.K. SHAW,AM, BUSHFIELD, $M$.; MACMILLAN, JL, MCNICOL,A (1985) Agonist-induced inositol phosphollipid metabolism and $\mathrm{Ca}+\mathrm{f}+\mathrm{Hux}$ in human platelet activation, Adw Exp.Med.Biol, 192:127-44.

[396] MAHIADEVAPPA, V.G., HOLUB,B.J. (1982)

The molecular species composition of individual diacyl phosphollipids in human platelets.

Biochin.Biophow wcta, $713 ; 73-9$.
[397]MAERUSP.W. WILSOND.B , CONNOLLY,T.M.,BROS5, T.E, NEUTELDE.J. (1985) Phosphoimositide turnower provides link in stimuhuresponse coupling. Trends Biochem $5 c$, 10, 1687 .

[398] MAKSYMIW,R, SULS.F, GAUB,H, SACK MANN,E. (1987) Electrostatic coupling of spectrin dimers to phosplivatudylserine containing lipid lamellae. Biochemistry, 26: $2983-90$.

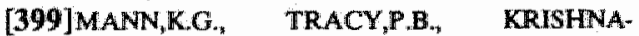
SWAMY, SENNY, R., ODEGAARD,B.H., NESHEIM,M.E (1987) Platelets and coagulation. im: "Thormbosis and Hatemostasis 1087" (Verstraete, $M$. Ver-

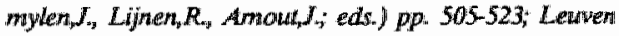
Uniwersily Press, Lewen.

[400]DE MARCO,L, GIROLAMI,A., ZMMMER MAN,TS, RUGGERI,ZM. (1986) won Willebrand factor interaction with the glycoprotein $\mathrm{I}_{b} / \mathrm{I}_{\mathrm{a}}$ complex. Ins role in platelet function as demonstrated in patients

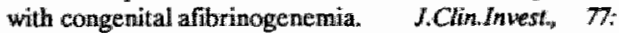
$1272-7$.

[401] MARCHEST, V.T. (1985) Stabilizing infrastructure of cell membranes. Annu.Rev. Cell Biol, , $53.1-61$.

[402] MARCHEST, V.T. (1984) Structure and function of the erythrocyte membrane skeleton.

Prog Clin.Biol Res, 159: 1-12

[403] MARGUERIE, G.A.,

GINSBERG,M.H., PLOW,E.F. (1987) The platelet fibrinogen receptor. in: "Plateles in Biology and Pathology, WT" (Macintyre, D.E. Gordon,J.L.," eds.) Res.Monogr.Cell Tissue Physiol, 13: 95-125, Amsterdam: Elsevier.

[404] MARINETTI,G.V. CRAIN,R.C. (1978) Topology of amino-phospholipids in the red cell membrane. J.Supranol.Stnuct, 8: $197-213$.

[405]MARKEY,F, PERSSON,T, LINDBERG,U. (1981) Characterization of platelet extracis before and after stimulation with respect to the possible role of profilactin as microfilament precursor. Cell, $23: 145-53$.

[406] MARTIN,O.C., PAGANO,R.E. (1987) Transbi layer movement of fluorescent analogs of phosphatidylserine and phosphatidylethanolamine at the plasma membrane of cultured cells. Evidence for proteinmediated and ATP-dependent process(es).

J. Btol:Chem, 202: 589028 .

[407] $\mathrm{MAUCO}_{3} \mathrm{G}_{*}, \quad$ DANGELMAIER, C.A. SMTTH,J.B. (1984) Inositol lipids phosphatidate and diacyldycerol share stearoylarachidonoylglycerol as a common backbone in thrombin-stimulated human platelets. Biochem J, 224:933-40.

[408】MCEVOY,L, WILLLAMSON,P, SCHLEGEL,R.A. (1986) Membrane phospholipid asymmetry as a determinant of erythrocyte recognition by macrophages. Proc.NatlAcadSciUSA, 83: 3311-5.

[409]MCGOWAN,E.B, DETWILER,T.C. (1986)

Modified platelet responses to thrombin. Evidence for two types of receptors or coupling mechanisms.

J.Biol.Chem, 261: 739-46.

[410] MCGOWAN,EB, DING,A, DETWLER,T.C. (1983) Correlation of thrombin-induced glycoprotein $\mathrm{V}$ hydrolysis and platelet activation. J.Biol.Chem, 258* $11249-8$ 
[411] MCGOWAN,E.B., YEO,KT., DETWLER;T. (1983) The action of $\mathrm{Ca}^{2+}$-dependent protease on platelet surface glycoproteins. Arch.Biachem.Biophyss. 227: 287.301.

[412]MCGREGOR,J.L, BROCHIER, $]_{2}$, WILD, $F_{4}$, FOLLEA,G, TRZECIAK,MC, JAMES, E, DECHAVANNE,M , MCGREGOR,L, CLEMETSON,K.J. (1983) Monoclonal antibodies against platelet membrane glycoproteins. Characterization and effect on platelet function. EurJ.Biochem, 131: $427-36$ n

[412a] MCGREGOR,J.L., MCGREGOR,L., BAUER,A.S., CATIMEL,B., BROCHIER,J, DECHAVANNE,M., CLEMETSON,KJ (1986) Identification of two distinct regions within the binding sites for fibrinogen and fibronectin on the $\mathrm{I}_{\mathrm{b}}-\mathrm{I}_{\mathrm{a}}$ human platelet membrane glycoprotein complex by monoclonal antibodies P2 and P4. EurJ.Biochem., 159: 4439.

[413] VAN MEER,G., OP DEN KAMP,J.A. (1982)

Transbilayer movement of various phosphatidylcholine species in intact human erythrocytes. J.Cell.Biochem., 19: 193-204.

[414] MELLONI,E., PONTREMOLI, $S$, MICHETTI,M., SACCO,O., SPARATORE, $\mathrm{B}_{\text {, }}$ HO RECKER,B.L. (1986) The involvement of calpain in the activation of protein kinase $C$ in neutrophils stimulated by phorbol myristic acid. J.Biol.Chem., 261: 41015.

[415] MELLONI,E., PONTREMOLI,S.; MICHETT],M., SACCO,O., SPARATORE,B., SALAMINO,F., HORECKER,B.L. (1985) Binding of protein kinase C to neutrophil membranes in the presence of $\mathrm{Ca}^{2+}$ and its activation by $\mathrm{a} \mathrm{Ca}^{2+}$-requiring proteinase.

Proc.NatlAcad.Sci.USA, 82: 6435-9.

[416] MELLGREN,R.L. (1988) On the mechanism of binding of calpastatin, the protein inhibitor of calpains, to biologic membranes. Biochem.Biophys.Res.Comm mum., 150: $170-6$.

[417]MELLGREN,R.L., MERICLE,M.T., LANE,R.D. (1986) Proteolysis of the $\mathrm{Ca}^{2+}$-dependent protedse inhibitor by myocardial $\mathrm{Ca}^{2+}$-dependent protease.

Arch Biochem. Biophys., 246:2339.

[418] MENASHI,S., WEINTROUB,H., CRAWFORD,N ( 1981$)$ Characterization of human platelet surface and intracellular membranes isolated by tree flow electrophoresis. J.Biol.Chem., 256: 4095-10\%.

[419]MEYER,M, HERRMANN,FH. (1984) Unusual electrophoretic behaviour of human blood platelet membrane glycoprotein Itb due to complex formation?

Thromb.Res., 35: 359-64.

[420]MIDDELKOOP,E, LUBIN,B.H., BEVERS,E.M., OP DEN KAMP, J.A.F, COMFURIUS, $P_{\text {, }}$ CHIU,D.T.-Y., ZWAAL,R.F.A., VAN DEENEN,L.L.M., ROELOFSEN,B. (1988) Studies on sickled erythrocytes provide evidence that the asymmetric distribution of phosphatidylserine in the red cell membrane is maintained by both ATP-dependent translocation and interaction with membrame skeletal proteins. Biochim Biophys Acta, 937: 281-8.

[421] MTTCHELL,C.A., SALEM,H.H. (1987) Cleavage of protein $\mathrm{S}$ by a platelet membrane protease. J.Clin.Inwest., 79: 3749.
[422] MOHANDAS, N, CHASIS, JA, SHOHET, SB. (1983) The influence of meimbrane skeleton on red cell deformability; membrane material properties, and shape. Semin Hernatol, $20: 225-42$.

[423] MOHANDAS,N., ROSSI,M, BERNSTEIN,S, BALLAS,S, RAVINDRANATH,Y, WYATT, MENTZER, W. (1985) The structural organization of skeletal proteins influences lipid translocation across erythrocyte menbrane. Jial Chem, 260: 142648.

[424]MOHANDAS, $\mathbb{N}_{\text {; }}$ WYATT, , $_{\text {, }}$ MEL,S.F, ROSSI, M.E., SHOHET,S.B. (1982) Lipid translocation across the human erythrocyte membrane: Regulatory factors. J.Biol.Chem., 257; 6537-43.

[425]MOMBERS, C, DE GIER,J, DEMEL,R.A., VAN DEENEN,L.L.M. (1980) Spectrin-phospholipid interaction. A monolayer studly. Biochim. Biophys.Acta, 003 : $52-62$.

[426] MOMBERS, C, VERKLEU, $A J$, DE GIER,J, VAN DEENEN,L.L.M. (1979) The interaction of spectrinmactin and synthetic phospholipids. II. The interaction with phosphatidylserine. Biochim. Biophys.Acta, 551: 271-81.

[427]MOOSEKER,M.S, COLEMAN,T.R, CONZEL, MAN,K.A. (1985) $\mathrm{Ca}^{2+}$ and the regulation of cytoskeletal assembly, structure and contractility. Ciba Found.Symp, 122; 232-49.

[428]MORROT,G., CRIBIER,S, DEYAUX,P.F., GELDWERTH,D, DAVOUST,J., BUREAU, J.F, FELLAMNN,P., HER VE,P., FRILLLEY,B. (1986)

Asymmetric lateral mobility of phospholipids in the human erythrocyte membrane.

Proc.Natl.Acad.Sci.USA, 83: 68637.

[429]MORSE,P.D. RUHLIG,M , SNIPES,W., KEITH,A.D. (1975) A spin-label study of the viscosity profile of sarcoplasmic reticular vesicles.

Anch Biochem.Biophys., J60: 40-56.

[430]MUHLRAD,A., ELDOR,A., KAHANE,I. (1978) Distribution of myosin, actin and actin-binding protein in membrane and soluble fraction of human blood pllatelets. FEBS Leth, $92(85-8$.

[431] MURACHI,T (1985) The proteolytic systen involving calpains. Biochem. Soc. Trans, 13: 1015-8

[432] MURACHI, T. (1983) Calpail and calpastatin.

Trends Biochent. Sci., $8: 167-9$.

[433] MURACHI, T, TANAKA,K, HATANAKA,M. MURAKAMI,T (1980) Intracellular $\mathrm{Ca}^{2+}$-dependent protease (calpain) and ith high-molecular-weight endogenous inhibitor (calpastatin). Adv Enzyme Regul, 19: 407-24.

[434]MüRER,E,H. (1985) The role of platelet $\mathrm{Ca}^{2+}$. Semin. Hematol, 22: 313-23.

[435] MuRER,E.H. (1976) Effects of lluoride on blond platelets. An overview. Flworide, 9: 173-84.

[436]MURER,E.H., DANIEL,J.L. (1983) Fluoride-unduced secretion from human blood platelets. Fhoride 16: $152-61$.

[437]MÜRER,E.H., DAY,HJ., LIEBERMAN,J.E. (1974) Metabolic aspects of the secretion of stored compounds from blood platelets. III. Effect of NaF on washed plateletts. Biochion.Biophys.Acta, 362: 266-75. 


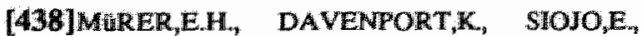
DAY,HJ. (1981) Metabolic aspects of the secretion of stored compounds from blood platelets. The effect of Natl at different pH on nucleotide metabolism and function of washed platelets. Biochen.J, 194: 187-92.

[439]NACHMAN,RL, SILVERSTEIN,R, ASCH,A.S. (1987) Thrombospondin: cell biology of an adhesive gycoprotein. "Thrombosis and Hemoxtasis 1987" (Verstraete, $M$, Vemyilen, $J$, Linen, $R_{\text {, Amout }} J_{\text {; }}$ eds.) $p p$. 81-91, Leuven Univensity Pness, Leunert.

[440]NACHMIAS, VT (1983) Platelet and megaka ryocyte shape change: triggered alterations in the cytoskeleton. Semin Memarol, 20: 201-81.

[441] N.ACHMIAS, V.T. (1980) Cytoskeleton of human platelets at rest and after spreading. I.Cell Biol, 86 : 7955802

[442] NACHMIAS, V.T, SULLENDER, J.S LON, J. (1979) Effiects of local anesthetics on human platelets: filopodial suppression and endogenous proteolysis. Blood, 53:63-72.

[443] NAKASHMMA,s, HATTORI, H, SHIRATO,L, TAKENAKA,A, MOZAWA, Y. (1987) Differential sensitivity of arachidonic acid release and 1,2-diacylgilycerol formation to pertussis toxin, GDP- $\beta-5$ and $\mathrm{NaF}$ in saponin-permeabilized human platelets: possible evidence for distinct GTP-binding proteins involving phospholipase $\mathrm{C}$ and $\mathrm{A}_{2}$ activation. Biocherm Biophys.Res. Commun., 148: 97/-8.

[444]NAKASHIMA, S, NAGATA,K, UEEDA, KK, NOZAWA,Y. (1988) Stimulation of arachidonic acid release by guanine necleotide in saponin-permeabilized neutrophils: evidence for involvement of GTP-binding protein in phospholipase $A_{2}$ activation.

Anch.Biochern.Biophys., 261: 375-83.

[445] NAKASHIMA, 5 , TOHMATSU, $T$, HATTO$\mathrm{RI}_{n} \mathrm{H}_{\text {, }}$ SUGANUMA,A., NOZAWA, Y. (1987)

Guanine nucleotides stimulate arachidonic acid release by phospholipase $A_{2}$ in saponin-permeabilized human pllatelets. J.Biochem., 101: 1055-8.

[446] NAKATA,T, HIROKAWA,N. (1987) Cytoskelelial reorganization of human platelets atter stimulation revealed by the quick-fiteeze deep-elch technique.

I.Cell Biol, 105: 1771-80.

[447] NAKANO, T, TERAWAKI,A, ARITA,H. (1987) Signal ransduction in collagen-stimullated rat platelets is composed of three stages. J.Biochen, 101: 116980.

[448]NAKANO,T, TERAWAKI,A. ARITA, H. (1986) Mensurement of thromboxane A2-induced elevation of ionined $\mathrm{Ca}^{2+}$ in collagen-stimulated platielets with the photoprotein, aequorin. J.Blochem, 92: 1285-8

[449] NARITA,H, PARK,HJ, TANAKA,K, MAT. SUURA, $T_{*}$, KITO,M. (1985) Insufficient mobilization of $\mathrm{Ca}^{2+}$ by early breakdown of phosphatidylinositol 4,5 bisphosphate for aggregation of human platelets by colllagen, J.Biochem, 98: 1063-8.

[450] NATHAN, $I_{\text {, }}$ FLEISHER, G, DVILANSKY,A, LIVNE, A., PAROLA,A.H. (1980) Membrane dynamic alterations associated with activation of human platelets by thrombin. Biochim. Bloplips.4cta, 598 417-21.
[451] NATHAN, I, FLEISCHER, G, LWNE,A, DVTLANSKY,A. PAROLA,A.H. (1979) Membrame microenvironmental changes during activation of human blood platelets by thrombin. A study with a fuorescent probe. IHiolChem, 254: 9822-8

[452]NEER,EJ $]_{w}$ CLAPHAM,DE. (1989) Rales of Gprotein subunüs in transmembrane signalling. Noture, 333: $129-134$.

[453] NELSON, WJ. TRAUB,P. (1982) Intermediate (10 nm) filament proteins and the $\mathrm{Ca}^{2+}$-activalted proteinase specific for vimentin and desmin in the cells from fish to man: an example of evolutionary conservation. J.Cell Sci, 57: 25-49.

[454] NIEDERMAN,R., POLLARD,T.D. (1975)

Human platelet myosin. II. In vitro assembly and structwre of myosin fillamients. J. Cell Biol, 67:72-92.

[455] NIEUWENHUIS,H.K, AKKERMAN,].W. HOUDIJK, W.P., SIXMA,J.J. (1985) Human blood platelets showing no tesponse to collagen fail to express surface glycoprotein $\mathrm{I}_{\text {. }}$. Natune, 318:470.2

[456] NIEUWENHUIS,H,K, SAKARIASSEN,KS ${ }_{\text {. }}$ HOUDIIK, W.P., NIEVELSTEIN,P.F, SIXMA,J.J (1986) Deficiency of platelet membrane glycoprotein $I_{\text {a }}$ associated with a decreased platelet athesion to suber dothelium: a defect in platelet spreading. Blood 68 $692-5$

[458] NIEWIAROWSK,S. (1987) Secreted platelet proteins. in" "Haemostasis and Thmombosis" (Bloom,A.L., Thomas,D.P,; eds.) pp. 90100, C7urekill Livingstome, Edinbught.

[458] NIGGLI, V., BURGER,M.M. (1987) Interaction of the cytoskelleton with the plasma membraine.

J.Membr.Biol, $100: 97 \% 121$.

[459] NISHIZUKA, Y. (1988) The molecular heterogemeity of protein kinase $C$ and its implications for cellular regualation. Nature, 334:661-5.

[460] NISHIZUKA, Y. (1986) Studies and perspectives of protein kinase C. Science, 233: 305-12.

[461] NISHIZUKA, Y. (1984) The role of protein kinase-C in cell surface signal transduction and tumor promotion. Nanre, 308 693-8.

[462] NISHIZUKA, Y, TAKAI, Y, KISHIMOTO,A, KIKKAWA,U., RAIBUCHI,K. (1984) Phospholipid turnover in hormone action. Recent.Prog Horm Res., 40 : $301-45$.

[463] NIXON,R A. (1986) Fodrin degradation by $\mathrm{Ca}^{2+}$. activated neutral proteinase (CANP) in retinal ganglion cell neurons and optic glia: preferential localization of CANP activities in neuroms. J.Neumosci, 6 1264-71.

$[464] \mathrm{NIXON}, \mathrm{R}, \mathrm{A}$, QUACKENBUSH, R, MTTO,A. (1986) Multiple $\mathrm{Ca}^{2+}$-activated neutral proteinases (CANP) in mouse retinal ganglion cell neurons: specificities for endogenous newronal substrates and comparison to purified brain CANP. J.Neurosci, \& 1252-63.

[465]NOMURA,M, SOBUE, K. (1987) Caldesmon regulates the three-dimensional contraction (myosin-dependenl contraction of the actin binding protein-induced actin gel). Biochem.Biophys.Res. Commmn. 144: 93643. 
[466] NURDEN,A.T. (1987) Abnormalities of platelet glycoproteins in inherited disorders of platelet function. in: Platelets in Biology and Pathology, IT (MacinIne,DE. Gordon,JL.; eds.) Res.Monogr.Cell Tisste Phywiol, 13: 37-93. Amstendam: Elsevier.

[467] NURDEN,A.T. (1987) Platelet membrane glycoproteins and their clinical aspects. in: "Thrombosis and

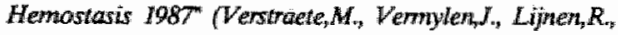
Amoutul.; eds.) pp. 93-125; Leuwen University Press. Leuven..

[468] NURDEN,A.T., CAEN,J.P. (1979) The different glycoprotein abnomalities in thrombasthenic and Bernard-Soulier platelets. Semin.Henatol, 16:234-50.

[469] NURDEN,A.T., DUPUIS,D., PIDARD,D, KUNICKI,T., CAEN,J.P. (1981) Biochemistry and immunology of platelet membranes with reference to glycoprotein composition. Ann.NYAcad Sici, 370: 72-86.

[470] OHALLORAN,T, BECKERLE,M.C, BURRIDGE,K (1985) Identification of talin as major cytoplasmic protein implicated in platelet activation. $\mathrm{Na}$ ture, 317: 449-5I.

[471] OHTAKE,N. (1986) Attachment of cytoskeletons to cell membranes in human blood platelets as revealed by the quick-freezing and deep-etching replica method. J. Uhtrastmuct.Mol.Strict.Res., 95: 84-95.

[472] OKTTA,J.R., PIDARD,D., NEWMAN,P.J., MONTGOMERY,R.R, KUNICKI,T $J$. (1985) On the association of glycoprotein $I_{b}$ and actin-binding protein in human platelets. J.Cell Biol, $100: 317.21$.

[473] OKUMURA, $T$, JAMIESON, G,A. (1976) Pladelet glycocalicin. I. Orientation of glycoproteins of the human platelet surface. J.Biol Chem., 251: 5044-9.

[474]ONJI,T., TAKAGI,M, SHIBATA,N. (1987) Calpain abolishes the effect of filamin on the actomyosin system in platelets. Biochim Biophys_Acta, 912:283-6.

[475]ONJ,T, TAKAGI,M., SHIBATA,N. (1985)

Filamin inhibits actomyosin ATPase activity in platelet. Biochem. Biophys. Res. Commun., 132: 307-12.

[476]OP DEN KAMP,JA.F. (1979) Lipid asymmetry in membranes. Anniu. Rew. Bhochem. 48. 47-71.

[477]OP DEN KAMP, JA,F ${ }$ ROELOFSEN, B, VAN DEENEN,L.L.M. (1985) Structural and dynamic as peas of phosphatidylcholine in the human erythrocyte membrane. Thends Biochem.Sici, 10:320-3.

[478]O'ROURKE,F.A, HALENDA,S.P., ZAVOIm CO,GB, FEINSTEIN,M.B. (1985) Inosital 1,4,5-trisphosphate releases $\mathrm{Ca}^{2+}$ from a $\mathrm{Ca}^{2+}$ transporting membrane vesicle fraction derived from human platelets. J.Biol. Chem., 260: 950-02.

[479] PAGANO,R.E., SLEBGHT,R.G. (1985) Emerging problems in the cell biology of lipids. Twends Biochert Sci, $10: 421-5$.

[480]PAINTER,R.G., GINSBERG,M.H. (1984) Centripettal myosin redistribution in thrombin-stimulated platelets. Relationship to platelet Factor 4 secretion. Exp. Cell Res., 155: 198-212

[481] PAINTER,R.G, GAARDE, W. GINSBERG,M.H. (1985) Direct evidence for the interaction of platelet surface membrane proteins GPII and III with cytoskeletal components: protein crosslinking studies. J.Cell. Biochem., 27:277-90.
[482] ]PANTER, R.O, PRODOUZ,KN, GAARDEW. (1985) Isolation of a subpopalation of glycopiconiein $\mathrm{I}_{b^{2}}$ III from platelet membranes that is bound to membrane actin. J.Cell Buol, $100 \times 6527$.

[483]PALEKJ. (1984) Disorders of red cell thembratse

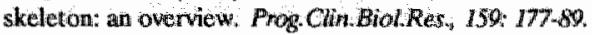

[484]PALEK, LU,S.C. (1980) Red cell numbrane skeleton: structure-function relationships.

ProgoClist Biol Ress, 43: 2I 44

[485]PANT,H.C.,GAINER, H. (1980) Properties of a $\mathrm{Ca}^{2+}$ activated protease in squid axoplasm which selectively degrades neurollanent proteins. J.Newnobiol. $I I$ : 1. 12 .

[486]PAPENHUYZEN, IISSER,A.J.W, (G., (1983)

Simulation of convoluted and exact emmission anison tropy decaly profiles. Biophys. Chem, 17, 57-65.

[486a] PARISE,L.V. PHILLIPS,D.R. (1986) Fibronectin-binding properties of the purified platelet glycopro-

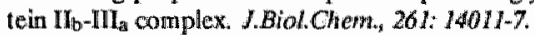

[487] VAN PARIDON,P,A, SMITH,R.L, WIRTZ,K.W.A. VISSER,AJ,W,G. (1988) A fuorescence decay study of parinaroyt-phosphatidylinositol incorporated into artificial and natural membrames.

Eur.Biophys.l., 16:53-67.

[488]PARKER,R.I., GRALNICK,H.R. (1986) Identifcation of platelet glycoprotein $\mathrm{II}_{\mathrm{b}} / \mathrm{II}_{\mathrm{a}}$ as the major bind ing site for released platelet-von Willebrand factor [published erratum appears in Blood 1987 Jun; $69(6): 1789$ Blood 69: 732-6.

[489]P.AYNE,M.R, RUDNICK,S.E. (1985) Tropo myosin. Structural and functional diversity. Cell.Mwscle Motil., 6: 141-84.

[490] PEERSCHKE,E.1 (1986) Platelet membrane alterations incluced by the local anesthetic dibucaine.

Blood, 68: 463-71.

[491] PEERSCHKE,EI.B. (1985) The platelet fibrinogen receptor. Sem.Hematol, 22: 241-59.

[492]PELEG, I, MUHLRAD,A ${ }_{\text {, }}$ ELDOR, A, GROSCHEL-STEWART,U, KAHANE,I. (1984) Charaterization of the ATPase activities of myosins isolated from the membrane and the cytoplasmic fractions of humam platelets. Arch.Biochern Biophys, 234" 42.53.

[493]PERIDES,G., KUEHW, S., SCHERBARTH, A., TRAUB; P. (1987) Probing of the structural stability of vimentin and desmin-lype intermediate tilaments with $\mathrm{Ca}^{2+}$-activated proteinase, thrombin and lysinie-specific endoproteinase Lys-C. EurJ.Cell Biol, 43:4508.

[494]PERRET,B, CHAP,H, DOUSTE-BLAZYL. (1979) Asymmetric distribution of arachidonic acid in the plasma membrane of human platelets. A determination using purified phospholipases and a rapid method for membrame isolation. Biochim, Biophys Acla, 556 : 434.46 .

[495]PHILLIPS,D.R. (1985) Receptors for platelet agonists. in "Platelet membrante gitropnoteins"

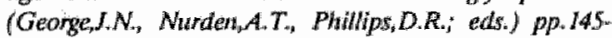
169, New York: Plemin Press.

[496] PHILLIIPS,D,R, AGIN,P.P. (1977) Platiclet plas. ma membrane glycoproteins. Evidence for the presence of nonequivalent disulfide bonds using nonreduced-re duced two-dimensional gel clectrophoresis. .

J.Biol.Chem, 252:2121-6. 
[497]PHILLIPSDIR CHARO,IF PARISELV FITZCERALD, LA (1988) The platelet membrane gycoprotein Ib Ith complex Blood, 7I: 831-43.

[498] PHILLIPG, D.R.

FTTZCERALD,LA., CHARO,1.F., PARISE,ILV. (1987) The platelet membrane fycoprotein If fII $_{\mathrm{a}}$ complex Sructure, function, and relationship to athesive protein receptors in nu cleated cells. Amin NYAcad Sci. 509, 177-87.

[499]PHILLIPS,D.R, JAKABOWA,W. (1977) Ca $\mathrm{Ca}^{2+}$ dependent protease in human platelets. Spectific cleav. age of platelet polypeptides in the presence of added $\mathrm{Ca}^{2}$. Jiol Chem, 252:5602-5.

[500]PHILLIPS, D.R.

JENNINGS,L.K, BERMDT,M.C. (1982) Studies of inherited bleeding disorders to identify platelet membrane glycoproteins inwolved in adhesion and aggregation.

Prog Clin Biol.Res, $97: 151-63$.

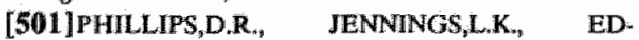
WARDS,H.H. (1980) Idemtification of membrane proteins mediating the interaction of human plateletsi.

$\checkmark$ Cell Biol. 86: 77-86.

[501a]PILON,M, JORDI,W, DE KRUIJFF,B, DEMEL R.A. (1987) Interactions of milochondrial precursor protein apocytochrome $c$ with phosphatidylserine in model membranes. A monolayer study. Biochinn BiophyswActa, 902: 207-16.

[S02]PISCHEL K.D.;

BLUESTEIN,H.G, WOODS, V.L.JR (1988) Platelet glycoproteins $\mathrm{I}_{\mathrm{a}}$, Ic, and $\mathrm{II}_{\mathrm{a}}$ are physicochemically indistinguishable from the wery late activation antigens adhesion-related proteins of lymphocytes and other cell types. J.Clin.Inwest., 81 : 505-13.

[503]PLOW,E,F, MARGUERIE, G., CINSBERG,M. (1987) Fibrinogen, fibrinogen receptors, and the peptides that inhibit these interactions. Brochem. Phamna col, 36: 4035-40.

[504] POLL, C, KYRLE, P:, WESTWICK, (1986) Ac tivation of protein kinase $\mathrm{C}$ inhibits sodium fluoride-induced elevation of thman platelet cytosolic free $\mathrm{Ca}^{2+}$ and thromboxane B2 generation. Biochem. Bio phys. Rew. Commum, 136.381 .9 .

[505] POLL, C., WESTWICK, (1086) Phorbol esters modulate thrombin-operated $\mathrm{Ca}^{2+}$ mobilisation and dense granule release in human platelets. Biochim.Biophyw 4 cta, $886: 43440$.

[506] POLLARD,T.D. (1981) Cytoplasmic contractile proteirus. J. Cell Biol, $9 M^{4}$ 156s-165s.

[507] POLLARD,T.D, COOPER, J, (1986) Actin and actin-binding proteins. A critical evaluation of mechanismus and functions. Anum. Rev Biochem, 55:987-1035.

[5M8] POLLOCK,W.K, IRVINE,R.F. RINK,T.J. (1986) Free $\mathrm{Ca}^{2+}$ requirements of agonist-induced thromboxane $A_{2}$ synt hesis in human platelets. EurJ.Phamacol, 132. 309. 12 .

[509] POLLOCK,W.K., RNN,TJ. (1986) Thrombin and ionomycin can raise platelet cytosolic $\mathrm{Ca}^{2+}$ to micromolar levels by discharge of internal $\mathrm{Ca}^{2+}$ stores! studies using fura-2. Biochem Biophys.Res.Commun., 130. $3018-14$
[510] POLLOCK,W.K, RINK, T J, IR VINE,R.F. (1986) Liberation of [3H]arachidonic acid and changes in cytosolic free $\mathrm{Ca}^{2}$ in fura-2-loacted himan platelets stimnlated by ionomycin and collagen. Biochem J, 235: 869. 77.

[511] PONTREMOLI,S., MELLONI,E (1986) Extraly. sosomal protein degradation. AmminevBlochem, 55 . 455-8\%.

[512]PONTREMOLI, $S_{*}$ MELLON,, MICHETTI,M. SPARATORE,B, SALAMTNO,F., SACCO,O., HORECKER,BL. (1987) Phosphorylation and proteolyuic modification of specific cytoskeletal proteins in human neutrophils stimulated by phorbol 12-myristate 13-acctate. Proc. NallAcad Sci USA, 84: 36048

[513] PONTREMOLI,S, MELLOM, $\mathbb{E}_{\text {, }}$ MICHET. TH,M., SACCO,O., SALAMINO,F., SPARATORE,B., HORECKER, B. L. (1986) Biochemical responses in actwated human newtrophils mediated by protein kinase $\mathrm{C}$ and a $\mathrm{Ca}^{2+}$-requiring proteinase. S.Biol.Chem., 261 : 8309.13

[514] PONTREMOLII, $S_{u,}$ MELLONI,E, HORECKER,B.L. (1985) Regulation of mammalian cytosolic $\mathrm{Ca}^{2+}{ }_{\text {-requiring neutrall proteinases. }}$

Cum. Top.Cell.Regul, 27: 293-304.

[515] PONTREMOLI,S, MELLONI,E $T I, M_{*} \quad$ SALAMINO,F.; SPARATORE, $B_{*,}$ HORECKER,B.L. (1988) An endogenous activatior of the $\mathrm{Ca}^{2+}$-dependent proteinase of buman neutrophils that increases its affinity for $\mathrm{Ca}^{2+}$. Proc NatlAcadSriUSA, 85: $17.40-3$.

[516]PONTREMOLI, 5. MELLONI,E, MICHETTI,M., SPARATORE,B., SALAMINO,F., SACCO,O HORECKER, B.L. (1987) Phosphorylation by protein kinase $\mathrm{C}$ of a $20-\mathrm{kDa}$ cytoskeletal polypeptide enhances its susceptibility to digestion by calpain Ipublished erratum appears in Proc Natl Acad Sci USA 1988 Apr;85(7):2140] Proc. NallAcad Sci USA, 84: 398-40I.

[517] PONTREMOLI,S, SALAMINO,F, SPARATORE,B, MICHETTI,M, SACCO,O., MELLONI,E (1985) Following association to the membrane, human erythrocyte procalpain is comverted and released as fullly active calpa"n. Biochim Biophys Acia, 831: 335-9.

[518]POWLING,MJ, HARDISTY,R.M. (1985) Gly* coprotein $\mathrm{II}_{b}-\mathrm{III}_{\mathrm{a}}$ conplex and $\mathrm{Ca}^{2+}$ infux into stimulated platelets. Blood, 66: 731-4.

[519]PRENDERGAST,F.G., HAUGHLAND,R.P., CALLAHAN PJ. (1981) 1-(4-(trimethylamino)phenyl] 6-phenylhera-1,3,5-triene: synthesis, fluorescence propertics. and use as a Muorescence probe of lipid bilayers. Biochemistoy, 20: 7333-8.

[520]PRITCHARD,K, MOODY,CJ. (1986) Caldes. mon: almodulin-binding actin-regulatory protein. Cell Culcium, 7: 309227.

[521] PURDON,AD, DANIEL,I.L, STEWART,G $J_{\text {, }}$ HOLMSEN,H. (1984) Cytoplasnic free $\mathrm{Ca}^{2+}$ concentration in porcine platelets. Regulation by an intracellular nonmitochondriall $\mathrm{Ca}^{2+}$ pump and increase after thrombin stimulation. Biochüm.Biophys.Acta, 800: 17887. 
[522]PUSZKTN,E,G, JENKIINS,CS, ORES-CARTON, C., ZUCKER,M.B. (1985) Platelet cytoskeleton $\alpha$-actinin in mormall and thrombasthenic pllatelets: distribution and immunologic characterization.

J.Lab.Clin.Med, 105: 52-62

[523]PYTELA, $R$, PIERSCHBACHER,M.D., GINSBERG,M.H. PLOW,E.F., RUOSLAHTI,E. (1986)

Platelet membrane glycoprotein $\mathrm{I}_{b} / \mathrm{HI}_{\mathrm{a}}$ : member of a family of Arg-Gily-Asp-specific adthesion receptors.

Science, 231: 1559-62.

[524] RABELLINO,E.M. (1984) Biology of human megakaryocytes: recent developments. Prog.Hemast.* Thromb , 7: 151-66.

[525]RAO,G.H., PELLER,J.D., WIHTE,J.G. (1985)

Measurement of ionized $\mathrm{Ca}^{2}{ }^{2}$ in blood platelets with a new generation $\mathrm{Ca}^{2+}$ indicator.

Biochem.Bio-

phys.Res.Conmum, 132:652-7.

[526]RAVAL,PJ., ALLAN,D. (1984) Phospholipid asymmetry in the membranes of intact human erythrocytes and in spectrin-free microvesicles derived from them. Biochim.Biophys_Acta, 772: 192-6.

[527] RENGASAMY,A, FEINBERG,H. (1988) Inositol 1,4,5-trisphosphate-induced $\mathrm{Ca}^{2+}$ release from platelet plasma membrane vesicles. Biochem.Biophys.Res. Commun., 150: 1021-6.

[528]RENGASAMY, SOURA,S, FEINBERG, H, (1987) Platelet $\mathrm{Ca}^{2+}$ homeostasis: $\mathrm{Na}+\mathrm{Ca}^{2+}$ exchange in plasma membrane vesicles.

Thromb.Haemost, 57: 337-40.

[529] VAN RUN,JL., GOVERS-RIEMSLAG,J.W., ZWAAL,R.F., ROSING,J. (1984) Kinetic studies of prothrombin activation: effect of factor $\mathrm{Va}$ and phospholipids on the formation of the enzyme-substrate complex Biochernistry, 23: 4557-64.

[530] VAN RLNN,J., ROSING,J, VAN DIEIJEN,G. (1983) Activity of human blood platelets in prothrombin and in factor $X$ activation induced by ionophore A23187. EurJ Biochent, 133: 1-10.

[531]RILEY,JR.,W.W., PFEIFFER,D.R. (1985) Relationships between $\mathrm{Ca}^{2+}$ release, $\mathrm{Ca}^{2+}$ cycling, and $\mathrm{Ca}^{2+}$-mediated permeability changes in mitochondria.

J.Biol.Chem, 260: 12410-25.

[532]RINK, T.J. (1986) Stimulus-response coupling mechanisms in human platelets. Agents Actions [Stppi], 20. $147-69$.

[533] RINK, T J, (1988) (news and views) A real receptor-operated $\mathrm{Ca}^{2+}$ channel? Nature, 334: 649-50.

[534] RINK, T J, HALLAM, T J. (1984) What turns platelets on? Trends Biochem.Sci., 9:215-9.

[535]RINK,T.J., SMITH,S.W., TSIEN,R. Y. (1982) Cytoplasmic free $\mathrm{Ca}^{2+}$ in human platelets: $\mathrm{Ca}^{2+}$ thresholds and $\mathrm{Ca}$-independent activation for shape-change and secretion. FEBS Lett., 148:21-6.

[536]RITCHIE, J.M. (1971) The mechanism of action of local anesthetic agents. in: "International Encyclopedia of Pharmacology and Therapeutics. Section 8: Local Anesthetics, vol. I" (Lechuth, $P_{\text {.; }}$ ed.) pp.: 131-66.

[536a] RTTTENHOUSE,S.E, ALLEN,C.L. (1982) Synergistic activation by collagen and 15 -hydroxy-9 $\alpha, 11 \alpha$ peroxidoprosta-5,13-dienoic acid (PGH2) of phosphatidylinositol metabolism and arachidonic acid release in human platelets. J.Cins.Invest, 70: 1216-24.
[537]RITTENHOUSE-SIMMONS,S., DEYKIN,D. (1981) Release and metabolisin of arachidonate in human platelets. Rex.Monographis Cell Tissue Physiol, 5. 34972 "Platelets in Biclogy and Pathology wol. $2^{4}$ (Gordion,J.L.; ed.) ElsevieriNonfh-Holland.

[538] RITTENHOUSE,S.E, HORNE, W.C. (1984) Ionomycin can elevate intraplatelet $\mathrm{Ca}^{2+}$ and activate phospholipase $\mathrm{A}$ without activating phospholipase $\mathrm{C}$. Biochem Biopthys. Res. Communt, 123: 393.7.

[539]ROELOFSEN,B, ZWAAL,R.F.A. (1976) The use of phospholipases in the determination of asymmetric phospholipid distribution in membrames.

Meth.Membr.Biol, 7: 147-77.

[540]ROELOFSEN,B., ZWAAL,R.F, WOODWARD,C.B. (1974) The action of pure phospholipases on native and ghost red cell membranes. Muthods Enzzy mol. 32: $131-40$.

[541]ROSENBERG,S,

LAWRENCE,J, STRACHER,A. (1982) Effect of various extraction solutions and thrombin activation on the composition of the platelet cytoskeleton. Cell Motil., 2: 317:32.

[542] ROSENBERG,S., STRACHER,A. (1982) Ellect of actin-binding protein on the sedimentation properties of actin. J.Cell Biol, 94: 51-5.

[543]ROSING, J., BEVERS,E.M., COMFURIUS,P., HEMKER,H.C., VAN DIEIJEN,G., WEISS, HJ, ZWAAL,R.F. (1985) Impaired factor X and prothrombin activation associated with decreased phospholipid exposure in platelets from a patient with a bleeding disorder. Blood, 65: 1557-61.

[543a]ROSING, J, VAN' RIJN,J.L., BEVERS,E.M., VAN DIEIEN,G., COMFURIUS,P., ZWAAL,R.F. (1985) The role of activated human platelets in prothrombin and factor $\mathrm{X}$ activation. Blood, 65: 319-32.

[544]ROSING, $J_{.}$, TANS, $G_{.,}$GOVERS-RIEMSLAG,J.W., ZWAAL, R.F., HEMKER, H.C. (1980)

The rolle of phospholipids and factor $\mathrm{Va}$ in the prothrombinase complex. J.Biol.Chent, 255: 274-83.

[545] ROTHMAN,J.E, LENARD,J. (1977) Membrane asymmetry. Science, 19:5: 743-53.

[546]]ROTMAN,A., HELDMAN,J., LINDER,S, (1982) Association of membrane and cytoplasmic proteins with the cytoskeleton in blood platelets. Biochemistry, 21: 1713-9.

[547]RUDA,E.M, PETTY,A, SCRUTTON,M.C., TUFFIN,D.P., MANLEY,P.W. (1988) Identification of small peptide analogues having agonist and antagonist activity at the platelet thrombin receptor.

Biocherr. Phamnacol, 37: 2417-26.

[547a] RUDA,E.M., SCRUTTON,M.C. (1987) Elfect of leupeptin on platelet agregation, fibrin formation and amidalysis induced by thrombin. Throntb. Res., 47:61I* a.

[548]RUGGIERO,M., LAPETINA,E.G. (1986) SUStained proteolysis is required for human platelet activation by thrombin. Thromb. Res. $42: 247-55$.

[549] SACKMANN, E. (1984) Physical basis of trigger processes and membrane structures. Biol.Memibranes, 5. 105-43 (Chapman, D.; ed.), London: Academic Press.

[550]SAGE,S.O., RINK,T.J. (1987) The kinetics of changes in intracellular $\mathrm{Ca}^{2+}$ concentration in furat 2 loaded human platelets. J.Biol.Chem., 262: 163649. 


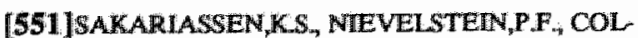
LER,B.S, SOXMA,J. (1986) The role of platelet

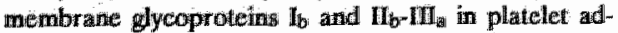
herence to human artery subendothelium. BrJ Hoema$10 k_{*}, 63: 681-91$.

[552]SAKON,M., KAMBAYASHI, OHNO,H, KOSANI,, . (1981) Two forms of Ca + + achivated neutral proteve in platielets. Thomb.Res, $24: 207-14$

[553] SALAMA,SE HASLAM,R. (1981) Subcelluar distribution of cyclic AMP-dependent protein kinase activity and of cyclic AMP binding proteins in human platielets. Modification by $\mathrm{Ca}^{24}$-dependent proteolysis. FEBS LEU, 130: $230-4$.

[554] SALZMANEW: JOHNSON,P.C., WARE, JA. (1965) Measurement of intracellular platelet $\mathrm{Ca}^{2+}$ with aequorin and Ooin: 2. Adw. Exp.Med Biol, 192:163-70.

$\llbracket 555]$ SAMIS J,A, ZBORIL, G.,ELCEJ.S. (1987) Cal pain I remains intact and intracellular during platelet activation. Immunochemical measurements with monocllonal and polyclonal antibodies. Biochem. $5.246: 481$. 8.

[556] \$ANCHEZ YAOUEE, I, LLANILLO,M. (1986) Lipid composition of subcellular particles from sheep platelets. Location of phosphatidylethanolamine and phosphatidylscrine in plasma membranes and plateler liposones. Biochim.Biophys.Acta; 856: 193-201.

[557]SANO,K, TAKAI, ${ }_{*}$, YAMANISHI,J, NISHIZUKA, Y. (1983) A role of $\mathrm{Ca}^{2+}$ activated phospholipid-dependent protein kinase in human platelel activation. Comparison of thrombin and collagen aclions. Jiol. Chem, 258, 2010-3.

[558]SANTORO,S.A, (1987) The dualistic nuechanisms of platelet adhesion to von Willebrand factor substrates. Exp. Cell Res., 173: 413-24.

[559]SANTORO,S.A. (1986) Identification of a 160,000 dalton platelet membrane protein that mediates the inithal divalent cation-dependent adhesion of platelets to collagen. Cell, 46. 913-20.

[560]SANTORO,5.A, CUNNINGHAM,L,W. (1981) The inter action of platelets with collagen. Res.Monographs Cell Thisue Plysiod, 5. 24064. mplateles in Biol ogy and Pathologg. voln2" (Gordoth, J.L.; ed.) Elsevier/Nonh-Holland.

[561]SANTORO,S,A., LAWING,W.J.,JR (1988) Competition for related but nonidentical binding sites on the glycoprotein IIb-III complex by peptides derived from platelat adhesive proteins. Cell 48 : $867-73$.

[562]S,ANTORO,S.A.

RAJPARA, S.M. STAATZ,W.D., WOODS, V.L.,JR (1988) Isollation and characterization of a platelet surface collagen binding complex related to VLA-2. Biochem Biophys Res. Com mun, $153: 217.23$

[563]SATO,C, NISHIZAWA, K, NAKAYAMA,T. NAKAMURA, $H_{\text {, }}$ YOSHIMURA, $N_{n}$, TAKANO,E: MURACHIT, (1986) Rapid proteolysis of brain MAP1 related cytoskeleton-associated $350 \mathrm{kd}$ protein by purified calpain. Cell.Struct.Funct, 11:253-7.

[564]SATO $\mathrm{S}_{\mathrm{B}} \mathrm{B}, \mathrm{OHNISH}, \mathrm{S}$.T. (1983) Interaction of a peripheral protein of the erythrocyte membrane, band 4.1, with phosphatidylserine-containing liposomes and erythrocyte inside-out vesicles. Eur, Biochem, 130: 19 . 25 .
[565]SAVART,Mi, BELAMR,M, PALLET,V, DUCASTAING,A. (1987) Association of calpains 1 and 2 with protein kinase C activities. FEBS Lett, 216: 22-6

[566] 1 SCHICK,E.P., SCHICK,P.K. (1986) Megaikaryocyte biochemistry. Semin. Hematok, 23: 68-87.

[567] SCHICK,B.P., SCHICK.P.K (1985) Cholesterd exchange in plateletis, erythrocytes and megakaryocytes. Brochim Blophys Acto, 833:281-90.

[568] ]SCHICK,P.K (1979) The role of platelet membrane lipids in platelet hemostatic activities. Semin.hematol, $16: 221-33$.

[569]SCHICK,PK, KURICA,KB, CHACKO,G.K (1976) Location of phosphatidylethanolamine and phosphatidylserine in the human platelet plasma membrane. J. Clin.linvest., 57: 1221-6.

[570]SCHLAEPFER,W.W., ZIMMERMAN,U] (1985) $\mathrm{Ca}^{2+}$-activated proteolysis of intermediate filaments. Amn.NYAcadSci. 455 . 552.62.

[571] SCHMAIER,A.H, SMTTH,P.M., PURDON,A.D, WHTTE,J G, COLMAN,R.W. (1986) High mollecular weight kininogen: localization in the unstimulated and activated pilatelet and activation by a platelet calpain(s). Blood, 67: 119-30.

[572] SCHNEIDER,E, HAEST,C.W, PLASA, G, DEUTICKE, B. (1986) Bacterial cytotoxins, amphotericin $B$ and local anesthetics enhance transbilayer mobility of phospholipids in erythrocyte membranes. Consequences for phospholipid asymmetry. Biochim.Bio physs Acta, 855: 325-36.

[573] SCHOLLMEYER,J.E. (1988) Calpain II involvement in mitosis. Science, 240: $911-3$.

[574]SCHOLLMEYER, J.E. (1986) Role of $\mathrm{Ca}^{2+}$ and $\mathrm{Ca}^{2+}$-activated protease in myoblast fusion. Exp Cell Res, $_{162: 411-22}$

[575]SCHOLLMEYER,J.E (1986) Possible role of calpain I and calpain II in differentiating muscle. Exp. Call Res: 163: 413\%22.

[576]SCHOLEY,J.M. TAYLOR,K.A., KENDRICKJONES, J. (1980) Regulation of non-muscle myosin assembly by calmodulin-dependent light chain kinase Nature, 287: 233-235.

[5771SCHROEDER,F. (1985) Fiuorescence probes unravel asymmetric structure of membranes. cell. Biochem, $11: 51-101$.

[578]SCHROEDER,F. (1978) Differences in lluidity between bilayer halves of twour cell plasma membranes. Nature, $276: 528-30$.

[579]SCHROEDER,F.(1984) Fluorescent sterols: probe molecules of membrane structure and function. Progiglipid Res. 23: $97-113$

[580]SCHUURMANS STEKHOVEN,F, BONT. ING,S.L. (1981) Transport adenosine triphosphatases: properties and functions. Physiol Rev, 61: 1.76

[581]SCRUTTON,M.C., KNIGHT,D.E. (1987) Per* meabilised preparations: their use in studies of signal transduction mechanisms in: Platelets in Biology and Pathology" IIT (MacIntyre,D.E, Gardon,J.L.; eds.) Res.Monogr. Cell Tissue Physioll, 13. 467-92, Amsterdam: Elsevier.

[582]SCRUTTON,M.C, KNIGHT,D.E, NIGGLI, V. (1985) Permeabilized platelets and exocytosis. Adw.ExpMedBiol, 192* 171-83. 
[583]SCRUTTON,M.C., WALLIS,R.B. (1981) Catecholamine receptors. Res.Monographs Cell Tissue Physiol, 5: 179-210. "Platelets in Biology and Pathology. nol.2" (GondonJ.L.; ed) ElsevierNNonth-Holland.

[584]SCULUY,M.F., WEERSINGHE,KM., KAKKAR, V.V. (1985) Platelet interaction with the contact system of coagrulation. Adv.Exp.Med.Biol, 192:389298.

[585] SEIGNEURET,M., DEVAUX,P.F. (1984) ATPdependent asymmetric distribution of spin-labeled phospholipids in the erythrocyte membrane: relation to shape changes. Proc.Natl_Acad.Sci.USA, 81: 3751-5.

[586]SEIGNEURET,M, FAVRE,E, MORROT,G., DEVAUX,P.F. (1985) Strong interactions between a spin-labeled cholesterol amalog and erythrocyte proteins in the human erythrocyte membrane. Biochim.Biophys_Acta, 813: 17482.

[587]SEIGNEURET,M., ZACHOWSKI,A., HERMANN, A, DEVAUX,P.F. (1984) Asymmetric lipid fluidity in human erythrocyte membrane: new spin-label. evidence. Biochemistry, 23: 4271-5.

[588]SEILER,S.M., ARNOLD,A.J, STANTON,H.C. (1987) Inhibitors of inositol trisphosphate-induced $\mathrm{Ca}^{2+}$ release from isolated platelet membrane vesicles. Biochem Pharmacol, 36: 3331-7.

[589]SHADLE,PJ., BARONDES,S.H. (1984) PLAtelet-collagen adhesion: evidence for participation of antigenically distinct entities. J.Cell Biol., 99: 2048-55.

[590]SHADLE,P.J., GINSBERG,M.H, PLOW,E.F., BARONDES,S.H. (1984) Platelet-collagen adhesion: imhibition by a monoclonal antibody that binds glycoprotein Ilb. J.Cell Biol, 99: 2056-60.

[591] SHATTIL,S.J., BRASS,L.F. (1987) Induction of the fibrinogen receptor on human platelets by intracellular mediators. J.Biol.Chem., 262: 992-1000.

[592] SHATTILS S. J, BRASS, L F. (1985) The interaction of extracellullar $\mathrm{Ca}^{2+}$ with the platelet membrane gllycoprotein $\mathrm{II}_{\mathrm{b}}-\mathrm{III}_{\mathrm{a}}$ complex. Nouv.Rev.Fr.Hematol, 27:211-7.

[593]SHATTIL_S.J. COOPER,R.A. (1978) Role of membrane lipid composition, organization, and fluidity in human platelet function. Prog.Hemost.Thromb., 4: $59-86$

[594]SHATTIL,SJ., HOXIE,I.A., CUNNINGHAM,M, BRASS,L.F. (1985) Changes in the platelet membrane glycoprotein Ill III $_{\mathrm{a}}$ complex during platelet activation. J.Biol.Chem, 260: 11107-14.

[595]SHEETZ,M.P. (1983) Membrane skeletal dynamics: role in modulation of red cell deformability, mobility of transmembrane proteins, and shape. Semin.Hematol, 20: 175-88.

[596]SHIFFER,K.A., GOERKE,J., DUEZGUENES, N., FEDOR, J, SHOHET,S.B. (1988) Interaction of erythrocyte protein 4.1 with phospholipids. A monolayer and liposome study. Biochim.Biophys.Acta, 937: $260-80$.

[597] SIFFERT,W., AKKERMAN,J.W. (1987) Activation of sodium-proton exchange is a prerequisite for $\mathrm{Ca}^{2+}$ mobilization in human platelets. Nature, 325: 456 8.
[598]SILVTUS,I.R, MCMILLEN,DA., SALEY,N.D., JOST,P.C, GRIFFITH;O.H. (1984) Competition between cholesterol and phosphatidylcholine for the hyArophobic surface of sarcoplasmic reticulum $\mathrm{Ca}^{2+}-\mathrm{AT}$. Pase. Biochemistry, 23: 538-47.

[599] SIMAN,R, BAUDRY,M., LYNCH,G, (1985)

Regulation of glutamate receptor binding by the cytoskeletal protein fodrin. Nature, $313: 225-8$

[599a]SIMON,M.F, CHAP,H,, DOUSTE-BLAZY,L. (1984) Activation of phospholipase $C$ in thrombinstimulated platelets does not depend on cytoplasmic free $\mathrm{Ca}^{2+}$ concentration. FEBS Letr, $170: 43-8$

[600]SIXMA,JJ. (1987) Platelet adhesion in health and disease. in: "Turombosis and Hemostasis 1987 " Verstraete, M., Vermylen,J, Lijnen, $R_{\text {, }}$ Amout,J.; edr.) pp. 127-146," Leaven University Press, Lewwen.

[601] SIXMA J J. (1987) Role of blood plateletis, plasma proteins and the vessel wall in haemostasis. in "Haemostasis and Thrombosis" (Bloom,A.L, Thomas,D.P.; eds.) pp. 283-302, Churchill Livingstone, Edinburgh.

[602]SKAER,R.]. (1981) Platelet degranulation.

Res.Monographs Cell Tissue Physiol, 5: $321-48$ "Platelets in Biology and Pathology, wol.2" (Gordon,J.L.; wd) Elsevier/North Holland Bionedical Press.

[603]SLEIGHT,R.G., PAGANO,R.E. (1985) Transbilayer movement of a fluorescent phosphatidylethanolamine analogue across the plasma membranes of cultured mammalian cells. J.Biol.Chem., 260: 1146-54.

[604]SMILLIE,L.B. (1979) Structure and functions of tropomyosins from muscle and non-muscle sources.

Trends Biochem.Sci, 4: 151-4.

[605] SMITH,J.B., DANGELMAIER,C, PURDON,A.D., MAUCO,G. (1985) Regulation of platelet phospholipid metabolism. Adw.Exp.MedBiol., 192: 281. 91.

[606]SNYDER,S.L., SOBOCINSKY,P.Z. (1975) An improved 2,4,6-trinitrobenzenesulfonic acid method for the determination of amines. Anal.Biochem, 64: 284-8.

[607]SOLUM,N.O. (1985) Platelet thembrane proteins. Semin. Hesnatol, 22: 289-302.

[608]SOLUM,N.O., OLSEN,T.M. (1985) Effeds of diamide and dibucaine on platelet glycoprotein $I_{b}$. actin-binding protein and cytoskeleton. Biochim.Biophys Acta, 817: 249-60.

[609]SOLUM,N.O., OLSEN,T.M. (1984) Glycoprotein $I_{b}$ in the Triton-insolubile (cytoskeletal) fraction of blood platelets. Biochim. Biophys.Acra, 799: 209-20.

[610]SOSINSKI,J., SZPACENKO,A., DABROW. SKA,R. (1984) Potentiation of actomyosin ATPase activity by filamin. FEBS Lett., 178. 311-4.

[611]SPANGENBERG,P., HELLER,R., BOSIA,A., ARESE,P., TILL,U. (1984) Chemical modification of cyloskeletal proteins of human blood platelets by diamide. Thromb. Res., 36: 609 18 .

[612]VAN DER STEEN,A.T, TARASCHI,T.F., VOORHOUT,W.F., DE KRUIJFF, B. (1983) Barrier properties of glycophorin-phospholipid systems prepared by different mettiods. Biochim. Biophys.Acta, 733: \$1-64. 
[613]STEDNER,M., LUESCHER,E.F. (1984) Fluores cence anisotropy changes in platelet membranes during activation. Biochemistry, 23:247-52.

[614] STERNWEIS,P.C., OLMAN,A.G. (1982) Allminifim: a requirement for activation of the regulatory componeat of adenylate cyclase by fluoride.

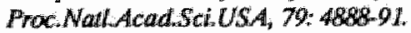

[615] STOSSEL, T.P. (1978) Contractile proteins in cell structure and function. Amuthev.Mad, 29; 427.57.

[616]STOSSEL,T.P. CHAPONNIER,C., EZZELL,RM, HARTWIG,J.H, JANMEY,P.A, KWIATKOWSKID.J, LIND,S.E, SMITH,D.B, SOUTHWTCK,F.S, YTN,HL, ZANER,KS. (1985)

Nonmuscle actin-binding protelins, Annu Rev,Cell Biol, I. $353-402$

[617] STRAUME,M, LITMAN,B.J. (1987) Equilibrium and dymamic structure of large, unilamellar, unstaturated. acyl chain phosphatidylcholine vesictes Higher oreder arralysis of 1,6 -diphenyl-1,3,5-hexatriene and $1-[4$ - (trimethylamonio) phenyl-6-phenyl-1,3, f-hexatriene anisotropy decay. Biachemistry, 26:5113-20.

[618]STRAUME,M. LITMAN, B. (1987) Infuence of cholesterol on equilibrium and dynamic billayer structwres of unsaturated acyl chain phosphatidylcholine vesicles as determined from higher order analysis of fluorescence anisotropy decay. Biochenisty: 26:5121-6.

[619]STRNAD,CF, WONG.K (1985) $\mathrm{Ca}^{2+}$ mobilization in fluoride activated human neutrophils.

Diochern.Bhophys. Res. Cornvawn, 133: 161-7.

[620]STUBBS,C.D, KINOSITA, K, JR, MUNKONOE, F ${ }^{-}$QUINN,P.J., IKEGAMI, A, (1984) The dynamics of lipid motion in sarcoplasmic tericulum. menibranes deternined by steady-state and time-resolved fhurescence measurements on 1,6-diphenyl1,3,5-hexatriene and related molecules. Biochim: Bio phys Acta, 775: 374-80.

[621]STUBBS,C.D., SMTTH,A.D. (1984) The modification of mamalian membrane polyunsaturated fatty acid composition in relation to membrane fluidity and function. Biochim. Biophys: Acta, 779: 89-137.

[622]SUGITA, H, ISHIIRA, S, KAMAKURA,K, NAKASE; $H_{n,}$ HAGIWARA, K, NONAKA, , TOMO-

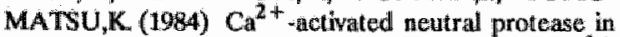
physiological and pathological conditions. in:" "Cat Regulotion in Biological Systems " (Ebashi, S., Endo,M.,

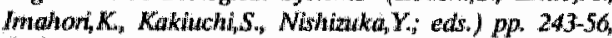
Tolyo: Academic Press.

[623]SUNE, A, BETTE-BOBILLO, $P_{5}$, BIEN VENUE, A, DELLLMANN, $P_{n}$, DEVAUX,P,F, (1987) Selective outside inside translocation of aminophos photipids in human platelets. Biochewistoy, 26. 2972-8

$[624]$ SUZUKa,K (1987) Ca ${ }^{2+}$ activated neutral protease: domain structure and activity regulation. Trestos Blochem.5ci, $12 ; 103 \mathrm{~s}$

[625]SUZUKI, $K_{2}$, HAYASHI,H, HAYASHI,T., IWAI, K (1983) Amino acid sequence around the active site cysteine residue of $\mathrm{Ca}^{2+}$-activated neutral protease (CANP). FEBS Lett, $152: 67-70$,

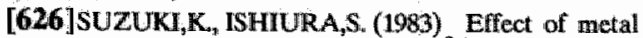
ions on the structure and activity of $\mathrm{Ca}^{2+}$-activated neutral protease (CANP). JBiochem $93: 1463-71$.
[627]SUZUKI, $K$, KAWASHIMA,S, IMAHORI, (1984) Structure and function of $\mathrm{Ca}^{2+}$-activated prom tease, in: "Ca" Regulation in Biological Systems:" (Eba-

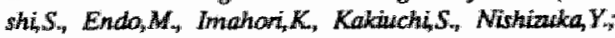
eds.) pp. 213-26. Tokyo: Acadernic Press.

[628]SUZUKI,K, TSUJ,S, ISHIURA,S., KIMURA, $Y_{1,2}$ KUBOTA,S, MAHORI,K (1981) Autdysis of $\mathrm{Ca}^{2+}$ activated neutral protease of chicken sheletal muscle. J.Biowihem, 90: 1787-93.

[629] SUZUKI, $_{4}$, TSUII, 3 , KUBOTA, $\mathrm{S}_{\text {, }}$ KIMU-

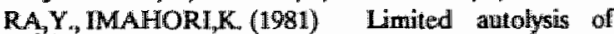
$\mathrm{Ca}^{2+}$-actryated meutral protease (CANP) changes its sensitivity to $\mathrm{Ca}^{2+}$ ions. J.Biochem, $90: 275-8$.

[630]TAKANO,E, PARK,Y.H., KITAHARA,A., YAMAGATA, $Y_{\text {; }}$ KANNAGI, $R$, MURACHE $T$. (1989) Distribution of calpains and calpastatin in buman blood cells. Biochem. Int., 16: 391-5.

[631]TAKAMATSU, HORNE,MK,3D, GRAL NICK,H.R. (1986) Identification of the thrombin Te ceptor on human platelets by chemical crosslinking.

J.Clin. Inwest., 77: 302-8.

[632]TAKAI, $Y$, KAIBUCHI,K, TSUDA, $T_{.,}$HOSHIJIMA,M. (1985) Role of protein kinase C in transmembrane signaling. J.Cell.Biochem, 29: 143-55.

[633] TAKEYAMA, $Y_{\circ}$, NAKANISHI,H., URATSUJI, Y, KISHMMOTO,A., NISHIZUKA, Y, (1986) A $\mathrm{Ca}^{2+}$-protease activator associated with brain microsomal-insoluble elements. FEBS Lett., 194: 1704.

[634]TANAKA,K.I, OHNISHI S (1976) Heteroge. neity in the Duidity of intact erythrocyte membrame and its homogerization upon hemolysis Biochim.Bia phys.4cta, 426: 218-31.

[635]TANDON,N., HINES,A., JAMIESON,G.A. (1985) Purification and characterization of glycoprotein IV from human platelets. (abstract) Thomb.Haentost. 54: 6

[636]TAPLEY,P.M., MURRAY,A.W. (1985) Evidence that treatment of platelets with phorbol ester causes proteolytic activation of $\mathrm{Ca}^{2+}$-activated, phospholipid. dependent protein kinase. Eur. Biochem.. 151: 11023.

[637]TAPLEY,P.M., MURRAY,A.W. (1984) Platellet $\mathrm{Ca}^{2+}$-activated, phospholipid-dependent proteín kimase: evidence for proteolytic actiwation of the enyme in cells treated with phospholipase C1. Biochem.Bio phys. Res. Commun., 18: 83941 .

[638]TARVER,A.P., KING,W.G., RITTEN. HOUSE,S.E (1987) Inositol 1,4,5-trisphosphate and inositol 1,2-cyclic 4,5-trisphosphate are minor components of total mass of inositol trisphosphate in throrinbin-stimulated platelets. Rapid formation of inositol 1,3,4-trisphosphate, J.Biol,Chem., 262: 17268-71.

[639]DER TERROSSIAN,E, PRULIERE, G. D'ALBIS,A. (1983) Purification of a troponin II-like factor from pig platelet. FEBS Lett, 152: 202-6.

[640]TERWILLIGER,T.C, WEISSMAN,L, EISENBER $G_{*} D$. (1982) The structure of melittin in the form 1 crystals and its implications for melittin's lytic and surface activities. Biophys $J_{y}, 37: 353-61$.

[641]THOMPSON,N.T., SCRUTTON,M.C. (1985) Intracellular $\mathrm{Ca}^{2+}$ nuxes in human platelets.

EurJ.Biochent, 1147: 421-7. 
[642] TILOOCK,C.P, BALLY,M.B, FARREN,SB, CLLLIS,P.R, GRUNER,S.M. (1984) Cation-dependent segregation phemomiena and phase behavior in model membrane systems containing phosphatidylserine: influence of cholesteroll and acyl chain composition. Biochentistry, 23: 2696-703.

[643]TTLLEY,L, CRIBTER,S., ROELOFSEN,B., OP DEN KAMP,JA, VAN DEENEN,LIL (1986) ATPdependent translocation of amino phospholipids across the human erythrocyte membrane. FEBS Leto, 194: 217.

[644] TOLNAI,S., KORECKY,B. (1986) $\mathrm{Ca}^{2+}$-dependent proteolysis and its inhibition in the ischemic rat myocardium. Can J. Cardiol, $2 * 42-7$.

[645] TOYO-OKA,T. (1982) Phosphorylation with cyclic adenosine $3^{*}, 5^{*}$ monophosphate-dependent protein kinase renders bovine cardiac troponin sensitive to the degradation by $\mathrm{Ca}^{2+}$-activated neutral protease.

Biochem Biophys.Res. Commun, 107: 44.50.

[646] TREMBLAY,], HAMET,P. (1987) Cyclic nucleotides and $\mathrm{Ca}^{2+}$ in platelets. in:" "Platelets in Biolongy and Pathology, III (Macintyre,D.E, Gomdon, J.L.," eds.) Res.Monogr. Cell Tissue Physiol., 13: 432-65, Amsterdam: Elsevier.

[647]TREMBLAY,J., HAMET,P. (1984) Ca $\mathrm{Ca}^{2+}$-dependent proteolytic stimulation of adenylate cyclase in platelets from spontaneously hypertensive rats. Metabolism, 33: 689-95.

[648] TRUGLIA, J, STRACHER,A. (1981) Purifica. thion and characterization of a $\mathrm{Ca}^{2+}$ dependent sulfhydryll protease from human platelets. Biochem.Biophys.Res.Commuth, 100:814-22.

[649]TSIEN,R.Y., POZZAN,T., RINK, T.J. (1984)

$\mathrm{Ca}^{2+}$ activities and fluxes inside small intact cells as measured with intracellularly trapped chelators.

Adv. Cycl.Nucl.Prot.Phosphor.Res., 17: 535-41.

[650] TSIEN,R.Y., POZZAN,T, RINK,TJ. (1982) $\mathrm{Ca}^{2+}$ homeastasis in intact lymphocytes: cytoplasmic free $\mathrm{Ca}^{2+}$ monitored with a new, intracellularly trapped fworescent indicatior. J Cell Biol, a4: 325-34.

[651]TSUCHIDA,K, AIHARA,H, ISOGA,K, HA. NADA, K, SHIBATA, N. (1986) Degradation of myocardial structural proteins in myocardial infarcted dogs is reduced by Ep459, a cysteine proteinase inhibitor. Biol Chem. Hoppe Seyler, 367: 30-45.

[652] TSUN,S, IMAHORI,K (1981) Studies on the $\mathrm{Ca}^{2+}$-activated neutral proteinase of rabbit skeletal muscle. I. The characterization of the $80 \mathrm{~K}$ and the $30 \mathrm{~K}$ subunits. J.Biochem., $90: 233-40$.

[653] TSUNI,T, OSAWA,T. (1987) The carbohydrate moiety of human platelet glycocalicin: the structures of the major Asn-linked sugar chains. J.Blochem. 101 : 241-9.

[654]TSUI, T, OSAWA,T. (1986) Structures of the carbohydrate chains of membrane glycoproteins $\mathrm{I}_{b}$ and III of human platelets. ABiochem, 100:1387-98.

[655]TSUINAKA, T, SHIBA,E, KAMBAYASH,], KOSAKI,G. (1983) Purification and characterization of a low $\mathrm{Ca}^{2+}$ requiring form of $\mathrm{Ca}^{2+}$-activated neutral protease from human platelets. Biochem Int., 6: 71-80.
[656]TSUJINAKA, T, SAKON, K, KAMAYASHU, KOSAKI, G. (1982) Cleavage of cytoskeletal proteins by two forms of $\mathrm{Ca}^{2+}$ activated neutral proteases in human platelets. Thromb.Rews, 20140 -50

[657]TSUNEHSA,S, TSUI,T, TOHYAMA,H. OSAWA,T (1984) Interaction of human platelet membrane glycoproteins with collagen and lecturs. Bio chim Biophys Acta, 797: 109.

[658]TUSZYNSIT,G.P, DANELJ.L, STEWART,G. (1985) Association of proteins with the platelet cytoskeleton. Semim. Hematol, 22; 301-12.

[659]URATSUJI, $Y^{\prime}$, NARANISHI, KEYAMA,Y, KISHIMOTO, ${ }_{\text {, N }}$ NISHIZUKA, $Y$. (1985) Activation of cellular protein kinase $C$ and mode: of inhibitory action of phospholipid-interacting compounds. Biochem.Biophys Res.Commum, 130:654-61.

[660] VANDERHOEK,J,Y, FEINSTEIN,ME. (1979)

Local anesthetics, chlorpronazine and propranolol in hibit stimulus-activation of phospholipase $\mathrm{A}_{2}$ in homan platelets. Mol Phannacol, $16: 171 \mathrm{~s} 80$.

[661]VERHOEVEN,A.J, MOMMERSTEEG,M.E., AKKERMAN,J,W. (1984) Metabolic energy is required in human platelets at any stage during optical aggregation and secretion. Biochim.Biophys.Acia, 800 . 242-50

[662]VERHOEVEN,AJ, MOMMERSTEEG,M.E, AKKERMAN,J,W, (1986) Comparative studies on the energetics of platelet responses induced by different agonists. BiochemJ., 236: 879-87.

[663] VERHALLEN,P.F、 ${ }_{*}$ BEVERS,E.M. COMFURIUS, $P_{,}$, ZWAAL,R.F.A. (1988) Fluoride-dependent $\mathrm{Ca}^{2+}$-induced platelet procoagulant activity shows that calpain is inwolved in increased phospholipid transbilayer movement. Biochim Biophys Acta, $942: 150-158$.

[664] VERHALLEN,P.F, BEVERS,E.M. COMFURIUS,P., ZWAAL,R.F. (1987) Correlation between calpain-mediated cytoskeletal degradation and expression of platelet procoagulant activity. A role for the platelet membrane-skeleton in the regulation of metnbrane lipid asymmetry? Biochim. Biophysicta, 903: $20 \%-17$.

[665] VERHALLEN,P.F., DEMEL,R.A, ZWTERS,H, GISPEN,WH. (1984) Adrenocorlicolropic hormone (ACTH)-lipid interactions. Implications for unvolvement of amphipathic helix formation. Bochns:Biophys.Acta, 775: 246-54.

[666]VERKLEIJ,AJ, ZWAAL,R.F, ROELOFSEN,B., COMFURIUS, $P_{* *}$ KASTELIJN, $D_{n,}$ DEENEN,LL LAN (1973) "The asymmetric distribution of phospliolipids in the human red cell membranc. A combined study using phospholipases and frecke-etch electron microscopy. Biochind Bioptys Acta, 323: 178 93.

[667]VERMYLEN,J., BADENHORST,PN., DECK. MYN,H., ARNOUT,J. (1983) Normal mechanisms of platelet function. Chin. Harmatot, 12: 107-5:1.

[668]VICKERS, D, KINLOUGH RATHBONE, R.L MUSTARD,J.F. (1987) Phosphatidylinositol 4,5-bisphosphate is selectively retained by platelet-ribrin clots formed by thrombin. Biochem $J, 245(3): 649-53$.

[669] VITALAJ,JRNEFELT,J.(1985) The red cell surface revisited. Thends Biochem Sci., 10i: 392-5. 


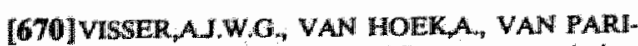
DON,P.A. (1987) Tine-resolwed fluorescence polarizat fion studies of parinar gyl phosphatidylcholine in Triton X-100 micelles and rat skeletall muscle membranes. int: "Membrate receptors, dynamics, and energethrs" (Wink,KW; ed); NATO ASI Sentes A, wol 133: 3536.1.

[671] VISSER,A,J,W,G, YKEMA,T, VAN HOEK,A

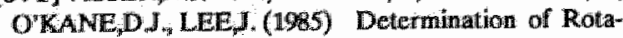
tional Correlation Thmes from Deconwoluted Fluorescence Amisotropy Decay Curves. Demonstration with 6,7-Dimethyl-8-ribihylumaxine and Lumazine Protein from Photobacterium leiogniathi as Fluorescent Indicators. Biochentiotry 24: 1480-06.

[672] VITTO,A, NIKON, R.A. (1936) Ca $\mathrm{Ca}^{2+}$-activated neatral proteinase of human brain: subunit structure and enzymatic propertes of multiple molecular forms.

J. Neurochem. 47: 1039-51.

[673] VOS,K, WAN HOEK,A., VISSER,AJ.W.G. (1987) Application of a reference convolution method to tryptophan fluonescence in proteins. A trefined description of rotational dynamics. Eur.J.Biochem. 165 : 55-63.

[674]WAGNER, GM., SCHWARTZ, $\mathbb{R}$ S., CHU,D.T. Y, LUBIN,B.H. (1985) Interaction of spectrin and band-4.1 with phosphatidylserine studied with model lipid systems, Clunics Haematol., 14:183-200.

[675]WALLACE, R,W, TALLAN",EA., MCMANUS,M.C (1987) Human platelet calmodulim-binding protcins: identification and $\mathrm{Ca}^{2+}$-dependent proteolysis upon platelet activation. Biochemisto, 26. 2766-73.

[676] WALSH,PN. (1985) Platelei-mediated coagulant protein interactions in hemostasis. Semin. Hewatol, 22 . 178-86.

[677]WWANG,C-T, SHLAO,Y.-J, CHEN, J,-C., TSAI,W.-J., YANG,C.-C. (1986) Estimation of the phospholipid distribution in the human platelet plasma membrane based on the effect of phospholipase- $A_{2}$ from Naja nigricollis. Biochim.Biophys.Acta, 856" 244 58.

[678]WANG,KK, VILLALOBO,A. ROUFO GALIS, B.D. (1988) Adivation of the $\mathrm{Ca}^{2+}$ ATPase of human erythrocyte membrame by an endogenous $\mathrm{Ca}^{2+}$ dependent neatral protease. Arch. Blochen.Blophys, 200: 6006704.

[679] WANG, L.L, BRYAN], (1981) Isolation of Ca $\mathrm{Ca}^{2+}$. dependent platelet proteins that interact with actin. Ceill 25: 63749 .

[6801WARE; I.A, JOHNSON,P.C. SMITH,M, SAILMAN,EW. (1986) Effect of common agonists on cytoplasmic ionized $\mathrm{Ca}^{2+}$ concentration in platelets. Measturement with 2-nethyl-methoxy \&-nitroquinoline (quin2) and aequorin. J.Cin.Jmuest, 77: 878-86.

[681] WARE,JA, SMITHMM SALZMAN,EW (1987) Synergism of platelet-aggregating agents. Role of ellev. ation of cytoplasmic $\mathrm{Ca}^{2+}$. J.Clin. Invest, 80: 267-71.

[682]WAXMAN, $\mathrm{L}$ (1981) $\mathrm{Ca}^{2+}$-activated proteases in mammalian tissues. Mevhods Enzymol, $80: 664.80$.

[683]WEIHING, R R. (1985) The Filamins" properties and functions. CanJ.Biochem. Cell Biol, $60.397-413$.

[684]WEIHING,R.R. (1979) The cytoskelloton and plasma membrane. Meth.4chicv. Exp.Pathot. 8: 2-109.
$[685]$ WEISS, H., VICIC,WJ, LAGES,BA, ROGERS, J. (1979) Isolated deficiency of platelet procoagulant activity. Am, $J$ Led, 67:206-13.

[686] WENCELDRAKE,J.D., PLOW,EF KL] NICKI,TJ, WOODS, VI, KELLER,D.M., GMSBERG,M.H. (1986) Localization of internal pools of membrane glycoproteins involved in platelet adhesive responses: Am J Pathol, 124:32434.

[687]WHEELOCK,MJ. (1982) Evidence for two structurally different forms of skeletal muscle $\mathrm{Ca}^{2+}$ activated protease. A.Biol Chem., 257: 12471-4.

[688] WHEELER,M.E, COX,A.C CARROLL,R.C. (1984) Retention of the glycoprotein $\mathrm{I}_{2}-\mathrm{IH}_{2}$ complex in the isolated platelet cytoskeleton. Effects of separable assembly of platelet pseudopodal and contractile cytos keletons. J.Cinn Invest, 74: 10809.

[689]WHEELER,ME., GERRARD,JM, CARROLL,R.C. (1985) Reciprocal transmembramous receptor-cytoskeleton interactions in concanavalin A-activated platelets. J.Cell Biol, 101. 993 m 1000 .

[690]WHIIE,G.C.,2D (1980) $\mathrm{Ca}^{2+}$ dependent proteins in platelets: response of $\mathrm{Ca}^{2+}$-activated protease in norand and thrombasthenic platelets to aggregating agents. Biochim. Biopitys Acta, 631." 1308.

[691]WHITE,G.C, 2D, KNUPP,C.L. (1985) Glycoprotein $\mathrm{V}$ hydrolysis by thrombin. Lack of correlation with secretion. Thromb.Res, 38: 641-8.

[692] WHITE, G.C. 2D, LEVINE,S.N, STEINER,AN. (1981) Platelet $\mathrm{Ca}^{2+}$-dependent proteins: identification and localization of the $\mathrm{Ca}^{2+}$-diependent regulator, calmodulin, in platelets. Ams.Hematol, 10. 359-67.

[693]WHITE,J.G. (1984) Arrangements of actin filalments in the cytoskelcton of human platelests. Am.J.Pathat, 117: 207 17.

[694]WHITE,J.G. (1979) Current concepts of platelet structure. AmJ.Clin.Pathol., 71: 363-78.

[695]WHITE,J.G. (1983) Ultrastructural modifications in platelet membranes and cytoskeleton following acti vation. Bhood Celts, 9: 237-61.

[696]WHITE H.G. (1987) Views of the platelet cytoskeleton at rest and at work. Ann. NY.AcadSci, 509: 156 . 76.

[697]WHITE $]_{n}$ G. (1987) An owerview of platelet structural physiology. Scanting Microsc., I: 1677-700.

[698]WHITE, J,G. GERRARD,JM. (1979) Interaction of microtubules and microfilaments in platelet contractile physiology. Meth A chiev.Exp.Pathol, a: 1-39.

[699] WHITE,J.G.,GERRARD,J.M. (1978) Platelet morphology and the ultrastructure of regulatory mecharisms involved in platelet activation. in: "Platelets: a multichisciplinay approach." (de Gaetano, $G_{\text {m. }}$ Garati"

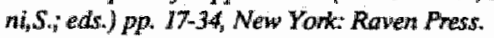

[700] WICKI,A.N, CLEMETSON,KIN (1987) The glycoprotein lo complex of human blood platelets.

EurJ.Biochern., 163: 13-50.

[701] WICKT,A N, CLEMET5ON,KJ. (1985) Structure and function of platelet membrane glycoproteins $I_{b}$ and V. Effects of leukocyte elastase and other proteases on platelets response to von Willebrand factor and thrombin. EurJ.Biochem, 153: 1-11. 
[702]WILLIAMSON,P, ANTIA,R., SCHLEGEL,RA. (1987) Maintenance of membrane phospholipid asymmetry. Lipid-cytoskeletal interactions or lipid pump? FEBS Lett, 210. 316-20.

[703]WILLIAMSON,P, BATEMAN,J, KOZARSKY,K, MATTOCKS,K, HERMANOWICZ $N$, CHOE,H.R., SCHLEGEL,RA. (1982) Involvement of spectrin in the maintenance of phase-state asymmetry in the erythrocyte membrane. Cell, 30:725-33.

[704] WILLIAMSON,P., SCHLEGEL,R. (1984) Maintenance of phospholipid asymmetry and its role in erythrocyte pathology. Pnog. Cin Biol Res., 159: 123-36.

[705]WILSON,D.B., CONNOLLY,T.M., BROSS,T.E., MAJERUS,P.W., SHERMAN,W.R, TYLER, A.N., RUBIN,LJ., BROWN,J.E. (1985) Isolation and characterization of the inositol cyclic phosphate products of polyphosphoinositide cleavage by phospholipase $C$. Physiological effects in permeabilized platelets and Limulus photoreceptor cells. J.Biol.Chern. 260: 13496 501.

[706] WILSON,DB., CONNOLLY,T.M, ROSS,T.S., ISHII,H., BROSS,TE, DECKMYN,H., BRASS,L.F., MAJERUS,P.W. (1987) Phosphoinositide metabolism in human platelets. Actv.Prostaglandin Thrombourane Leukorriene Res., 174: 558-62.

[707] WTSNIESKI,BJ., IWATA,K.K. (1977) Electron spin resonance evidence for vertical asymmetry in ani mal cell membranes. Biochemistry, 16:1321\%6.

[708]WOODS,C.M., LAZARIDES,E. (1988) The ery throid membrane skeleton: expression and assembly during erythropoiesis. Annu.Rev.Med., 30: 107-22.

[709] WOODS, V.L, JR, WOLFF WLE. KELLER,DM. (1986) Resting platelets contain a substantial centrally located pool of glycoprotein $\mathrm{IL}_{b}-\mathrm{II}_{\mathrm{a}}$ complex which may be accessible to some but not other extracellular proteins. J. Biol. Chem, 261: 15242-51.

[710]WROBLEWSKI,R., LA DUE,IIS. (1955) Spectrophotometric determination of lactate dehydrogenase activity. Pnoc soc.Ep.Biol.Med, 90:210-5.

[710a] WU, C. S.C, HACHIMORI,$A_{4}$ YANG, JT. (1982) Lipid-induced ordered conformation of some peptidle hormones and bioactive oligopeptides: predo minance of a-helix over $\beta$-form. Biochemistry, $21: 4556$ 62

[711] WYLER,B * BIENZ,D., CLEMETSON,KJ., LUSCHER,E.F. (1986) Glycoprotein $\mathrm{I}_{b}-\beta$ is the only phosphorylatted major membrane glycoprotein in human platelets. Biochem, 234:373-9.

[712] YAMAGUCH,A, YAMAMOTO,N, KITAGAWA, $H_{1,}$ TANOUE,K, YAMAZAKI,H, (1987) Ca $\mathrm{Ca}^{2+}$ influx mediated through the $\mathrm{GPII} / \mathrm{MII}_{\mathrm{a}}$ complex during pllatelet activation. FEBS Lett., 225: 228-32.

[713] YAMAMOTO,K., KOSAKI,G., SUZUKI,K, TANOUE, $K_{0}$, YAMAZAKI,H. (1986) Cleavage site of $\mathrm{Ca}^{2+}$-dependent protease in human platelet membrane giycoprotein Ilib. Thromb.Res., 43: 41-55.

[714] YAMAMOTO,K, YAMAMOTON, KUTAGAWA, H, $_{n}$ TANOUE,K, KOSAKI,G, YAMAZAKI,H. (1986) Localization of a thrombin-binding site on human platelet membrane glycoprotein $I_{b}$ determined by a monoclonal antibody. Thromb. Hotemast. 55: 162-7.

[715] YEAGLE,P.L. (1985) Cholesterall and the cell
[716] YEAGLE P.L (1984) Phospholipid protein interactions and the structure of the thaman erythrocyte membrane; nuclear magnetic rescunance studies.

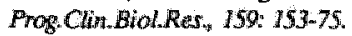

[717] YOSHDA K, KIMURA, (1985) Elfect of $\mathrm{Ca}^{2}$ on protein composition of human platelet cytos-

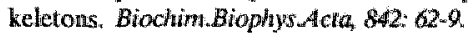

[718] YOSHIDA,K. KIMURA,H. (1984) Presence of calmodulin in buman plateltet cyitosikeletons and its concentration change tapon activation of platelets. Bio chins. Brophys Acta, 801: 2907.

[719]YOSHIDA,R., NACHMLA, Y.T. (1987) Phorbol ester stimulates $\mathrm{Ca}^{2+}$ sequestration in saponized human platelets. J.Biol.Chem, 262: 16040-54.

[720] YOSHIDA, N, WEKSLER, $B$, NACHMAN, $R$, (1983) Purification of human platelet $\mathrm{Ca}^{2+}$-activated protease. Effect on platelet and endothelial function. J.Biol.Chem., 258.7168-74.

[720a]DE YOUNG,L,R, DILL, K.A. (1988) Solute partitioning into lipid billayer membrames. Biochemistry. 27: 5281 -9.

[721]ZACHOWSKI,A, FAVRE,E, CRIBIER, S. HER VE,P., DEVAUX,P.F. (1986) Outside-inside translocation of aminophospholipids in the buman erythrocyte membrane is mediated by a specific enzyme.

Biochemisfry, 25: $2585-90$.

[722]ZACHOWSKI,A., HERRMANN,A., PARAF,A., DEVAUX,P.F. (1987) Phospholipid outside-inside translocation in lymphocyte plasma me mbranes is a protein-mediated phenomenon. Biochinn. Biophys.Acta, 897: $197-200$.

[723]ZHANG,Z, LAWRENCE,J, STRACHER,A. (1988) Phosphorylation of platelet actin binding protein protects against proteolysis by $\mathrm{Ca}^{2+}$ dependent sulfhydryl protease Biochem. Biophys. Res. Commun., 151: $355-60$.

[724]ZHUANG,Q.Q., ROSENBERG,S., LAWRENCE $J_{\text {, }}$ STRACHER,A. (1984) Role of actin binding protein phosphorylation in platelet grtoskeleton ats

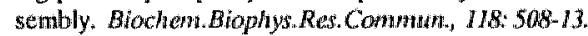

[725]ZILBERMAN, $Y_{\text {, }}$ GUTMAN, $Y_{*}$, KOREN, $R$. (1982) The effect of werapamil, lanthanum and local anesthetics of serotonin release from rabbit plateletw. Bhochin Bioplyys Acta, 69: 106-14.

[726]VAN ZOELEN,E]" DE KRUIJFF,B., NAN DEENEN,L.L. (1978) Protein-mediated transbilayer movement of lysophosphatidylcholine in glycophorincontaining vesicles. Biochiws. Biophys $4 c t a, 508: 97-108$

[727]ZUCKER,MB., NACHMIAS,V.T. (1985) Platelet activation. Amerioscterosis, $5: 2-18$.

[728]2WAAL,R.F.A. (1988) Scrambling membrane phospholipids and local control of blood clotting. News Int. Physiol.Sci, 3: 57.61.

[729]ZWAAL,R.F. (1978) Membrane and lipid irvolvement in blood coagulation. Biochim.Biophys Acta, 575: 163-205.

[730]ZWAAL,R.F.A, BENERS,E.M. (1986) Structural and functional aspects of the platelet plasma membrane. ins." "Lipids and Membranes: Pasi, Present and Future"

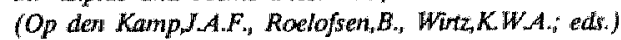
pp. 231-257, Anusterdam : ELsuvier. 
[731]2WAAL,F F, BEVERS,EM. (1983) PLatellet phospholipid asymmetry and its significance in bemostassis. Subcell.Biochem, 9. 292-334.

[732]2WAAL, F.F, BEVER,E.M F, COMFURIUS, (1986) Platelets and coagulation. in: Blood coagulation" (Zwrod, R. F.A., Hemker,H.C, eds.) pp. I4l. 169, Amsterdam Elsevier.

$[733] 2 W A A L, R, F, A$, BEVERS,EM, ROSINC, (1960) Structural aspects of the platelet membrane with empliassis on procoagulant phospholipids. in: "Btology" and Pathology of Platelet-Vessel Wall Interactions" ("ieds:) pp:247.265, New Yowk Academic Press.

[734]ZWAAL, R.F, COMFURIUS,P. HEMKER, H,C., BEVERS E.M. (1984) The inlabition of platelet prothrombinase activity by prostacyclin. Homostaris, 14: 3204.
[735] ZWAAL,R.F. HEMKER,HLC (1982) Blood cell meribranes and haemostasis. Haemostasis, $11_{*}^{*}$ 12-39.

[736]ZWAAL,R.F. ROELOFSEN,B, COMFURIUS,P, VAN DEENEN,LL (1975) Organization of phospholipids in human red cell membranes as detectied by the action of various purified phospholipases. Bio chum Biophys Acta, 406:83-96;

[737]. ZWAAI,R.F.A, ROSING, $J_{,}$, TANS, G. BEV ERS,EM, HEMKER,HC. (1980). Topologicall and kinetic aspects of phospholipids im blood coagulation. in:" "The Regulation of Coagulation" (Mann, Toylor, eds.) pp. 95-115, Amsilendam: Elsevier 


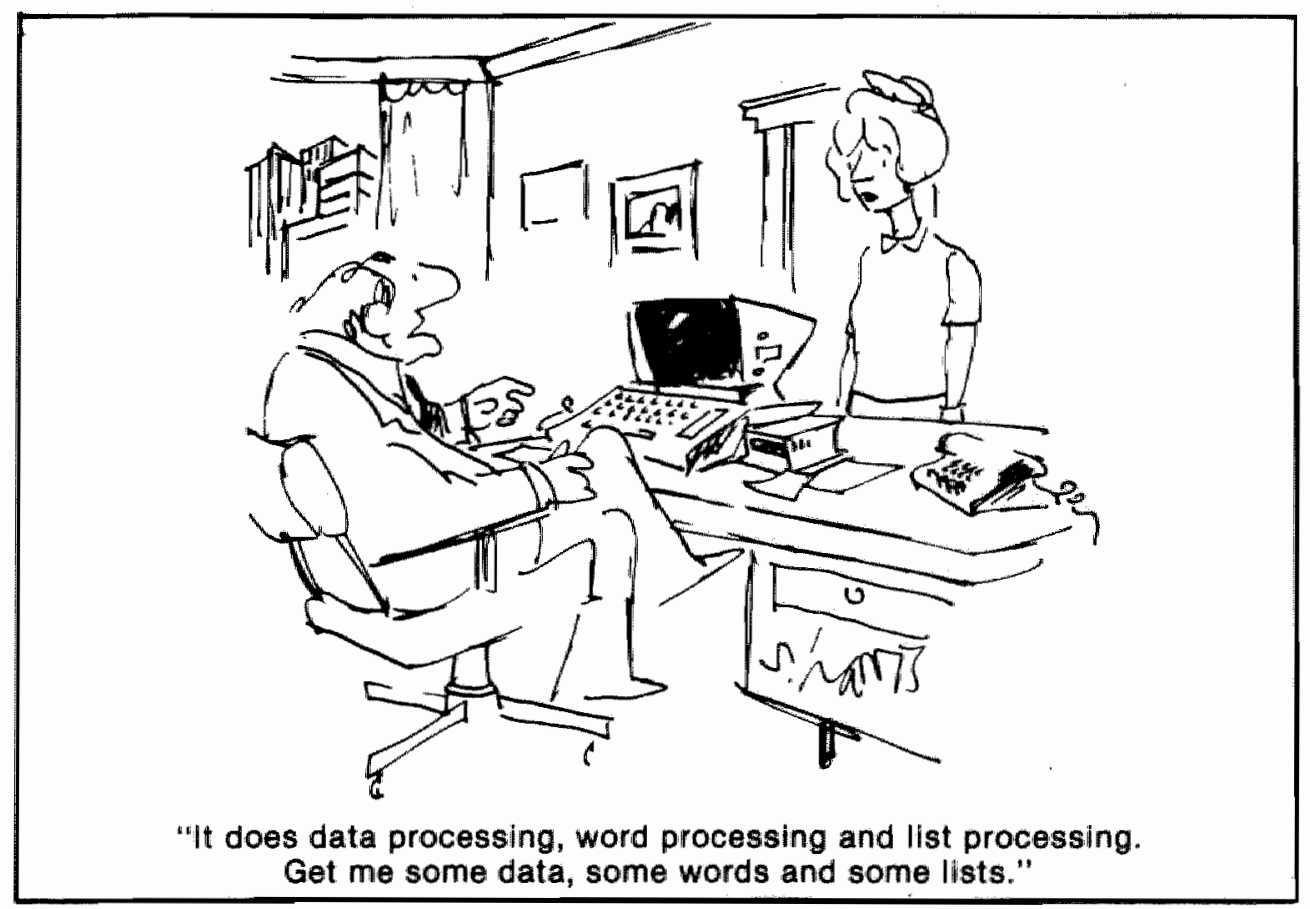




\section{SUMMARY}

Blood platelets play a multifaceted role in the arrest of bleeding upon vessel wall injury. Normally, platelets circulate in the blood stream as quiescent cells. Upon vessel wall injury, platelets become activated: they adhere to the uncovered subendothelial fibrils (adhesion), secrete their granular contents (rellease reaction), and stick to each other (aggregation) to form a primary hemostatic plug. The primary platelet plug is consolidated by fibrin, which is formed upon initiation of the coagulation cascade, a process which is dramatically accelerated by activated platelets. Finally, contractile mechanisms in platelets lead to clot contraction.

Research in the group of professor Zwaal is concentrates on the acceleration of coagulation by activated human blood platelets. This property of activated platelets is referred to as procoagulant activity, and can easilly be measured by the conversion of prothrombin to thrombin by the enzymatic complex of factor $X_{\mathrm{a}}$ and $\mathrm{V}_{\mathrm{a}}$, under conditions at which the procoagulant activity of the platelet is rate limiting. Routinely, purified coagulation factors are used, and the amount of thrombin formed is determined with a chromogenic substrate.

Not all platelet activators are equally potent in stimulation of procoagulant activity. Thrombin is a moderate and collagen is an efficient stimulator of procoagulant activity. Most potent, however, is the combination of collagen and thrombin. The condition of two powerful platelet activators could mean that coagulation is accelerated especially at the site of vessel wall injury.

Previous research showed that negatively charged phospholipids, like phosphatidylserine, are responsible for the procoagulant activity of the platelet surface. In a quiescent platelet, phospholipids are asymmetrically distributed over the plasma membrane (transmembrane asymmetry), in such a way that phosphatidylserine is wirtually confined to the intracellular side of the plasma membrane. This means that phosphatidylserine has to cross the plasma membrane during platelet actiwation. The molecular and cellular mechanisms involved in the transmembrane movement (flipflop) of phosphatidylserine have been the research subject of this thesis. Initial investigation of platelet protein patterns after gel electrophoresis showed that the potency of various platelet activators to induce procoagulant activity correlated with the extent of degradation of cytoskeletal proteins by a $\mathrm{Ca}^{2}$-dependent protease (calpain).

The involvement of the cytoskeleton in the expression of platelet procoagulant activity was further investigated by comparing the appearance of phosphatidylserine at the platelet surface with the degradation of cytoskeletal proteins by calpain, at various conditions (chapter 2). The rate of both processes was compared after treatment of platelets by a $\mathrm{Ca}^{2+}$ ionophore, the combination collagen and thrombin, and the local anesthetics dibucaine and tetracaine. It was found that on a time-scale of less than one minute, several minutes, and almost one hour, respectively, the generation of procoagulant activity followed an identical course as the generation of calpain activity. The observed similarity suggests that both activities are related. This suggestion was further investigated employing the $\mathrm{Ca}^{2+}$-dependence of calpain. Platelets were activated by a $\mathrm{Ca}^{2+}$ iono- 
phore, while extracellular $\mathrm{Ca}^{2+}$ was controlled with the aid of $\mathrm{Ca}^{2+}$ buffers. It was found that the $\mathrm{Ca}^{2+}$-dependence of calpain was almost identical with that of the appearance of procoagulant activity, again indicative of a relation between these processes.

A different approach to investigate the relation between flipflop of phosphatidylserine and degradation of cytoskeletal proteins by calpain was found in the use of fluoride-treated platelets. It appeared that during treatment with fluoride, platelets temporarily increase the permeability of their plasma membrane towards $\mathrm{Ca}^{2+}$. When these fluoride-treated platelets subsequently become exposed to $\mathrm{Ca}^{2+}$, their intracellular $\mathrm{Ca}^{2+}$ levels will rise to an extent determined by the current $\mathrm{Ca}^{2+}$ permeability of their plasma membranes. Comparison of the generation of procoagulant activity and calpain activity after addition of $\mathrm{Ca}^{2+}$ again showed a close resemblance. Convincing evidence for a relation between flipflop of phosphatidylserine and degradation of cytoskeletal proteins was obtained by addition of leupeptin, a specific inhibitor of calpain, during the fluoride treatment. It was found that inhibition of calpain resulted in inhibition of procoagulant activity.

Another clue for a relation between the structuur of the cytoskeleton and the asymmetric distribution of phospholipids over the plasma membrane was obtained with the aid of artificial vesicles, pinched off from the platelet plasma membrane (chapter 4). Addition of a synthetic lipid which spontaneously incorporates into the plasma membrane renders the membrane instable, resulting in extrusion of abundant lipid in the form of a plasma membrane vesicle (spicule). These spicules have lost their transmembrane lipid asymmetry. In addition, the composition of their cytoskeletal proteins has changed. These findings can be explained in terms of a relation between cytoskeletal organization and transmembrane lipid asymmetry.

The molecular mechanisms of transmembrane movement of phosphatidylserine during the generation of platelet procoagulant activity were investigated employing the fluorescent amphiphilic membrane probe TMA-DPH (trimethylammonium-diphenyll hexatriene). When TMA-DPH is added to suspended platelets, part of it will immediately incorporate into the outer leaflet of the plasma membrane where it strongly fluoresces, while most of it will stay in solution where it is not fluorescent.

At first, the fluorescence properties of TMA-DPH in unstimulated platelets were investigated employing advanced fluorimetric techniques (chapter 5). These techniques allow detailed determination of the structural properties of the membrane in the vicinity of TMADPH. After addition to unstimulated platelets, TMA-DPH will slowly diffuse into the platelet interior, which is evidenced by an increase in fluorescence due to uptake of TMA-DPH from the solution. Monitoring the fluorescence properties of TMA-DPH during its penetration into the platelet, showed that the structure of intracellular membranes is much more fluid than that of the outer leaflet of the plasma membrane. Comparison of the fluorescence properties of TMA-DPH in various model membranes suggested that an uneven distribution of cholesterol in the platelet is the cause of the observed differences in membrane fluidity. It is concluded that the outer leaflet of the plasma membrane contains about 3 times more cholesterol than the cytosolic leaflet.

Changes in the fluorescence properties of TMA-DPH during stimulation of platelets are described in chapter 6. Stimulation of platelets by a $\mathrm{Ca}^{2+}$ ionophore resulted in a rapid increase in fluorescence intensity to the level of lysed platelets. This finding indicates that when phosphatidylserine moves rapidly from the inner side to the outer side of the plasma menbrane, TMA-DPH moves rapidly into the platelet. This can be explained by assuming that during generation of platelet procoagulant activity the molleculair structure of the plasma membrane becomes locally disturbed. Along these membrane disturbances (flipsites) lipids and lipid-like molecules can rapidly move from either side of the plasma membrane to the other. Further information about the properties of these flipsites was obtained by investigation of their lifetime and $\mathrm{Ca}^{2+}$-dependence. Addition of TMA-DPH 1 minute after the $\mathrm{Ca}^{2+}$ ionophore showed that the flipsites were no longer present. Determination of the $\mathrm{Ca}^{2}$-dependence of the generation of flipsites, measured in the same way as the $\mathrm{Ca}^{2+}$-dependence of cytoskeletal degradation and procoagulant activity, showed a close similarity with the $\mathrm{Ca}^{2+}$ dependence of calpain.

In conclusion, the findings described in this thesis can be explained with the following 
model. In unstimulated platelets the transmembrane lipid asymmetry is dependent on a direct interaction of specific cytoskeletal proteins with anionic lipids of the plasma membrane. Stimulation of platelets by the combined action of collagen and thrombin will lead to activation of calpain. Limited proteolysis of cytoskeletal proteins by calpain results in loss of the stabilizing influence of these proteins on the membrane structure. As a consequence, the membrane lipids will immediately reorganize resulting in a loss of transmembrane lipid asymmetry. Phosphatidylserine, present at the platelet surface, can participate in the acceleration of blood coagulation. 


\section{SAMENVATTING}

Bloedplaatjes spelen een veelzijdige rol in het stelpen van bloedingen. Normaal gesproken wordt een bloedplaatje in een inactieve toestand meegevoerd door de bloedstroom. Zodra echter een bloedvat beschadigd wordt, raken bloedplaatjes geactiveerd: ze binden aan het blootgelegde bindweefsel (adhesie), scheiden de inhoud van hun gramula uit (release reactie), en klonteren samen (aggregatie) tot een primaire bloedprop. Tevens kunnen geactiveerde bloedplaatjes de bloedstolling versnellen. Het gevormde librine bind de bloedplaatjes aan elkaar tot een stevige prop (secundaire bloedprop), die samentrekt door een contractie proces in de bloedplaatjes.

Het onderzoek in de groep van professor Zwaal concentreert zich op het versnellen van de bloedstolling door geactiveerde humane bloedplaatjes. Deze eigenschap van geactiveerde bloedplaatjes wordt procoagulant activiteit genoemd, en kan gemakkelijk worden afgeleidt uit de snelheid waarmee prothrombine omgezet wordt door factor $\mathrm{X}_{\mathrm{a}}$ en $\mathrm{V}_{\mathrm{a}}$ in thrombine. Routinematig worden hiervoor gezuiverde stollingsfactoren gebruikt, en wordt de hoeveelheid gevormde thrombine bepaald met een chromogeen substraat.

Niet alle stimulatoren van bloedplaatjes zijn even goed in staat de procoagulant activiteit op te wekken. Thrombine is een matige en collageen is een goede stimulator van de procoagulant activiteit. De combinatie van collageen met thrombine vormt echter het sterkste fysiologische signaal woor het opwekken van de procoagulant activiteit. De noodzaak woor twee krachtige plaatjes activatoren zou kunnen betekenen dat de vorming van thrombine vooral versneld wordt door de plaatjes op de plaats van beschadiging van de vaatwand.

In het werleden is gebleken dat negatief geladen fosfolipiden, zoals fosfatidylserine, verantwoordelijk zijn voor de procoagulant activiteit. In een inactief bloedplaatje zijn de fosfolipiden asymmetrisch verdeeld over beide zijden van het plasma membraan (transmembraan asymmetrie), waarbij fosfatidylserine zich vrijwel uitsluitend aan de birnenkant van het plasma membraan bevindt, en dus tijdens de plaatjes activatie door het membraan naar buiten moet bewegen. De molecullaire and cellulaire processen betrokken bij de transmembraan beweging (flipflop) van fosfolipiden zijn onderwerp van het promotie-onderzoek geweest. Onderzoek naar veranderingen in eiwitpatronen met behulp van gelelectroforese, liet in eerste instantie zien dat de mate van procoagulant activiteit, opgewekt door verschil. lende plaatjes activatoren, correleerde met de mate van afbraak van cytoskelet eiwitten door een $\mathrm{Ca}^{2+}$ afhankelijke protease (calpaine) in het bloedplaatje.

De rol van het cytoskelet tijdens het opwekken van procoagulant activiteit in bloedplaatjes is nader onderzocht door het naar buiten komen van fosfatidylserine te vergelijken met de afbraak van cytoskeleteiwitten door calpaine, onder verschillende omstandigheden (hoofdstuk 2). Zo werd de vormingssnelheid van beide processen vergeleken na behandelling van bloedplaatjes met een $\mathrm{Ca}^{2+}$ ionofoor, met de combinatie collageen \& thrombine, en met lokale anesthetica (dibucaine, tetracaine). Het bleek dat op een tijdschaal van respectievelijk minder dan cén minuut ${ }_{\text {, }}$ enkele minuten, en bijna een uur, het opkomen van 
procoagulant activiteit en calpaine activiteit parallel liep. Deze correlatie suggereert dat beide activiteiten iets met elkaar te maken hebben. Die suggestie werd onderzocht door gebruik te maken wan de $\mathrm{Ca}^{2+}$ afhankelijkheid van calpaine. Bloedplaatjes werden geactiveerd met een $\mathrm{Ca}^{2+}$ ionofoor terwill extracellulair $\mathrm{Ca}^{2+}$ gemanipuleerd werd met behulp van $\mathrm{Ca}^{2+}$ buffers. Gevonden werd dat de $\mathrm{Ca}^{2+}$ afhankelijkheid van calpaine vrijwel identiek was aan die van het opwekken van procoagulant activiteit, hetgeen opnieuw suggereert dat deze processen met elkaar verbonden zijn.

Een andere manier om de relatie tussen flipflop van fosfatidylserine en afbraak van cytoskeleteiwitten te onderzoeken werd gevonden in bloedplaatjes behandeld met fluoride (hoofdstuk 3). Het bleek dat behandeling met fluoride in afwezigheid van $\mathrm{Ca}^{2+}$, tijdelijk de $\mathrm{Ca}^{2+}$ permeabiliteit van het plasma membraan verhoogt. Wanneer deze behandelde bloedplaatjes vervolgens worden blootgesteld aan $\mathrm{Ca}^{2+}$, zal dit $\mathrm{Ca}^{2+}$ naar binnen stromen en het plaatje activeren. Onderzoek naar onstaan van calpaine activiteit en procoagulant activiteit na toevoeging van $\mathrm{Ca}^{2+}$, liet weer zien dat beide parallel liepen. Een sterker bewijs voor een verband tussen cytoskelet afbraak en flipflop van fosfatidylserine werd verkregen door tijdens de fluoride behandeling een remmer van calpaine toe te voegen. Het bleek dat remming van calpaine resulteerde in remming van procoagulant activiteit, een duidelijke indicatie dat afbraak van cytoskelet eiwitten op een of andere manier verantwoordelijk is voor de flipflop van fosfatidylserine.

Een volgende aanwijzing voor een verband tussen de structuur van het cytoskelet en de transmembraan lipiden asymmetrie werd verkregen met behulp van kunstmatige membraan structuren, afgesplitst van het bloedplaatje (hoofdstuk 4). Door toevoegen van synthetisch lipid dat spontaan in het membraan incorporeert, wordt het plasma membraan onstabiel, en zal de overmat lipid afgesplits worden in de vorm een klein bolletje omsloten door het afgesplitste membraan (spicule). Het bleek dat in deze spicules de transmembraan lipiden asymmetrie verloren is gegaan. Dit gaat gepaard met een afwijking in de samenstelling van het cytoskelet, met name het eiwit myosine is vrijwel geheel afwezig in de spicules.

Inzicht in het moleculaire mechanisme van transmembraan beweging van fosfolipiden tijdens het procoagulant wrorden van bloedplaatjes vereiste een andere aanpak. Gekozen werd voor het gebruik van een fuorescent amfifiel molecuul, TMA-DPH (trimethylammonium-difenylhexatriën), als membraan sonde. Een klein gedeelte van het TMA-DPH dat toegevoegd wordt aan inactieve bloedplaatjes, wordt zeer snel opgenomen in de buitenste laag van het plasma membraan waar het fluoresceert, terwijl het merendeel in oplossing blijft: waar het niet fluoresceert.

In eerste instantie werd TMA-DPH in een niet-gestimuleerd bloedplaatje onderzocht met geavanceerde fluorescentie technieken (hoofdstuk 5). Met deze technieken is het mogellijk uit de fluorescentie eigenschappen van TMA-DPH af te leiden hoe de membraanstructuur in de omgeving van TMA-DPH er uit ziet. Na toevoeging aan bloedplaatjes zal TMA-DPH langzaam naar binnen diffunderen. Dit gaat gepaard met een geleidelijke toename in de fluorescentie intensiteit als gevolg van opname van TMA-DPH wit de oplossing. Door de fluorescentie eigenschappen van TMA-DPH te volgen terwijl het langzaam het bloedplaatje binnendringt, werd gevonden dat de membraanstructuur binnen in het bloedplaatje veel vloeibaarder is dan aan de buitenkant van het plasma membraan. Vergelijking van de fluorescentie eigenschappen van TMA-DPH in verschillende model membranen met bekende samenstelling, suggercerde dat een ongelijkmatige verdeling van cholesterol in het bloedplaatje de oorzaak moest zijn van de gevonden verschillen in vloeibaarheid. Hieruit werd de conclusie getrokken dat de buitenzijde van het plasma membraan ongeveer 3 maal zoveel cholesterol bevat als de binnenzijde.

Onderzoek naar veranderingen in fluorescentie eigenschappen van TMA-DPH als gevolg van bloedplaatjes activatie (hoofdstuk 6), liet zien dat activatie met de $\mathrm{Ca}^{2+}$ ionofoor een snelle toename in de fluorescentie intensiteit tot gevolg heeft, tot het niveau van gelyseerde plaatjes die totaal verzadigd zijn met TMA-DPH. Met andere woorden, tijdens activatie van bloedplaatjes door een $\mathrm{Ca}^{2+}$ ionofoor, waarbij fosfatidylserine snel over het plasma membrane naar buiten beweegt, beweegt TMA-DPH zich snel naar binnen toe. Dit kan verklaart worden door aan te nemen dat tijdens het opwekken van procoagulant activiteit in bloedplaatjes, de lipiden bilaag structuur van het plasma membraan op moleculair niveau verstoord raakt. Langs deze membraan verstoringen (flipsites) 
kunnen dan lipiden snel dwars over het membraan bewegen. Nadere informatie over de eigenschappen van de flipsites werd verkregen door onderzoek naar hun levensduur en de $\mathrm{Ca}^{2+}$ afhankelijkheid. Toevoeging van TMADPH aan bloedplaatjes één minuut ná de $\mathrm{Ca}^{2+}$ ionofoor, liet zien dat de flipsites niet meer aanwezig waren. De $\mathrm{Ca}^{2+}$ afhankelijkheid van de generatie van flipsites, gemeten op dezelfde manier als beschreven voor cytoskelet afbraak en procoagulant activiteit, kwam overeen met die van calpaine.

Concluderend, kunnen de hier beschreven waarnemingen met het volgende model verklaart worden. In een inactief bloedplaatje wordt de transmembraan lipiden asymmetrie mede gehandhaafd door interactie van bepaalde cytoskelet eiwitten met de lipiden bilaag. Stimulatie van bloedplaatjes door een combinatie van collageen en thrombine leidt tot activering wan calpaine, dat bepaalde cytoskelet eiwitten in fragmenten afbreekt. Hierdoor komt de stabiliserende invloed van die cytoskelet eiwitten te vervallen, en zal het membraan zich ogenblikkelijk reorganiseren, resulterend in een verlies van transmembraam lipiden asymmetrie. Het fosfatidylserine dat nu ook aan de buitenkant van het plasma membraan zit zall daar de bloedstolling kunnen versnellen. 


\section{DANKWOORD}

Is elke arbeid eigenlijk teamwerk, promoveren is dat in het bijzonder, omdat promoveren een opleidingsfase is. De opleiding tot zelfstandig wetenschappelijk onderzoeker vergt regelmatig positieve en negatieve terugkoppeling van de directe werkomgeving. In mijn geval waren dit Robert Zwaal, Edouard Bevers, en Paul Comfurius.

Rob, het was even aftasten in het begin. Vooral mijn eigenzinnige karakter zat onze samenwerking we] eens in de weg. Ik heb veel van je geleerd. Met name het gemak waarmee je een probleem dat de "werkvloer" bezighield relativeert in een groter geheel. Ik heb er bewondering voor.

Edouard, jou heb ik leren kennen als iemand met oog voor dletails, altijd bedacht voor een valkuil. Van jou heb ik geleerd dat je eigenlijk niet voorzichtig genoeg kunt zijn in het wetenschappelijk onderzoek.

Paul, van jou heb ik geleerd dat er een belangrijke kloof ligt tussen idee en verantwoorde uitwoering: de praktische details. Bovendien was jij mijn dagelijkse gesprekspartner.

Al het bloed voor de experimenten werd vrijblijvend afgestaan door vele "bewoners" van het BMC, iedereen wordt hartelijk bedankt. Voor het afnemen van al dat bloed stonden Mieke Janssen en Truus Janssen elke keer weer klaar, mijn oprechte dank daarvoor.

Tijdens mijn promotie-onderzoek heb ik twee studenten mogen begeleiden, Johan van der Palen en Maike Schemmann. Bedankt voor jullie inzer.

Het opzetten van een geautomatiseerd literatuursysteem was niet mogelijk geweest zonder de steun van Rob Zwaal, Coen Hemker, en Gerard Hornstra.

Jan Rosing wil ik bedanken voor het jarenlange gebruik van zijn personal computer, de eerste van Biochemie.
Deelname aan de "ETRO Course" 1986, te Maastricht, was een onvergetelijke ervaring die ik te danken heb aan Theo Lindhout en Coen Hemker, merci.

Op de plaats waar gebruikelijkerwijs de secretariele ondersteuning wordt bedankt, will ik mijn personal computer bedenken. Altijd was hij er als ik hem nodig had, zonder een vervelend woord of vermoeidheidsverschijnselen. Wat een domme-kracht!

Het zou echter onjuist zijn de secretariele ondersteuning onbetuigd te laten. Mariet Molenaar en Trees Camphuisen, zonder jullie zou ik nu nog niet een buitenlandse telefoonnummer kunnen draaien, of een reisdeclaratie kunnen invullen.

Paul Devilee, dankzij jouw hulp heb ik het manuscript van mijn proefschrift netjes kunnen laten printen op de HP.

Mensen die niet genoemd zijn hoeven zich niet gepasseerd te voelen. Ik heb het naar mijn

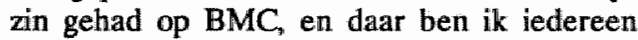
dankbaar voor.

Sylvia, zonder jouw steun als levenspartner, moeder, huisvrouw, tekenares, praatpaal, en wat dies meer zij, was het proefschrift nooit geworden wat het is. Ik denk dat ik in het bijzonder geboft heb met je geleidelijk steeds meer geprofileerde betrokkenheid bij mijn werk. Jij hebt me het gevoel gegeven dat ik het niet alleen voor mezelf gedaan heb maar voor ons hele gezin. Samen aan de toekomst werken is veel leuker.

Kinders, jullie hebben me keer op keer laten beseffen dat er meer is in het leven dan werken. Hoewel "papa achter het bureau" vaak realiteit was, hoop ik jullie niet tekort gedaan te hebben. 


\section{List of publications}

\section{PAPERS}

VERHALLEN, P.F.J., DEMEL, R.A., ZWIERS, H., GISPEN, W.H. (1984) Adrenocorticotropic hormone (ACTH)-lipid interactions. Implications for involvement of amphipathic helix formation. Biochim.Biophys.Acta, 775: 246-254.

VERHALLEN, P.F.J., COMFURIUS, P., BEVERS, E.M., ZWAAL, R.F.A. (1986) On the regulatory role of the cytoskeleton in the expression of platelet procoagulant activity Agen ts \& Actions (Suppl), 20:181-187.

VERHALLEN, P.FJ., BEVERS, E.M., COMFURIUS, P, ZWAAL, R.F.A. (1987) Correlation between calpain mediated cytoskeletal degradation and platelet procoagulant activity.Bio chim.Biophys.Acta, 903: 206-217.

BEVERS, E.M., VERHALLEN, P.F.J., LINSKENS, W.M.A. COMFURIUS, P., ZWAAL, R.F.A. (1987)Loss of phospholipid asymmetry in dilauroylphosphatidylcholine induced plasma membrane vesicles from human platelets. Biochim.Biophys_Acta, 903: 197-205.

BEVERS, E.M., VERHALLEN, P.F.J., ZWAAL, R.F.A. (1988)Organisation of phospholipids in the plasma membrane of human platelets in relation to changes in the cytoskeleton.in: "Biochemistry and Physiopathology of Platelet Membrane" (Marguer;, G., Zwaal, RF.A.; eds.) Colloque INSERM vol.158, pp. 1 10., Panis, J.Libbey Eurotext Ltd.
VERHALLEN, P.FJ., BEVERS, E.M., COMFURIUS, P., ZWAAL, R.F.A. (1988)Fluoridedependent calcium-induced platelet procoagulantactivity shows that calpain is involved in increased phospholipid transbilayer movement.

Biochim.Biophys.Acta, 942: 150-158.

VERHALLEN, P.F.J., COMFURIUS, $P$, BEVERS, E.M., ZWAAL,R.F.A. (1988) Cytoskeletal degradation by calpain results in flipflop of phosphatidylserine in the plasma membrane of activated blood platelets.in." "Structure and Function of the Cytoskeleton" (Rousset, B., ed.) Colloque INSERM., Paris, J.Libbey Eurotent Ltd, in press.

VERHALLEN, P.F.J., BEVERS, E.M., COMFURIUS, P, ZWAAL, R.F.A. (1988) De rol van de $\mathrm{Ca}^{2+}$-afhankelijke protease, calpaine, tijdens het procoagulant worden van humane bloedplaatjes.in: "Thrombosis \& Atherosclerosis: diagnostiek, preventie, en behandeling anno 1988" (Ten Cate, J.W., Kontmann, C.; eds.). Am* sterdam, Stichting Amstol, in press.

VERHALLEN, P.F.J., et.al. (1988) Transbilayer asymmetry of membrane fluidity and cholesterol in the plasma membrane of unstimulated platelets. A time-resolved fluorescence study employing trimethylammonium-diphenylhexatriene (TMA-DPH), with reference to model systems.Submitted.

VERHALLEN, P.F.J., et.al. (1988)Rapid loss of plasma membrane lipid asymmetry by calpain-dependent generation of flipsites during platelet activation. A fluorescence study with the membrane probe trimethylammonium-diphenylhexatriene (TMA-DPH) Submitted. 


\section{ABSTRACTS}

VERHALLEN, P.F.J., BEVERS, E.M., COMFURIUS, P., LINSKENS, W.M.A., ZWAAL, R.F.A. (1987) Calpain-mediated cytoskeletal degradation correlates with stimulation of platelet procoagulant activity. Thrombos.Hemastas., 58: 6.

BEVERS, E.M. VERHALLEN, P.F.J., COMFURIUS, P., LINSKENS, W.M.A., ZWAAL, R.F.A. (1987)Loss of phospholipid asymmetry in small vesicular structures pinched off from platelet membranes upon incubation with dilauroyllecithin. Thrombos. Hemostass, 58: 457.

VERHALLEN, P.F.J., BEVERS, E.M., ZWAAL, R.F.A. (1988)Involvement of flipsites and membrane skeleton in regulation of transbilayer movement of phosphatidylserine in the platelet plasma membrane. Proceedings of the FEBS Advanced Course "Lipid Flow", March 612, Maria Alm, Austria Abstract no. 2-13., Utrecht, published by the University of Utrecht.
VERHALLEN, P.F.J., COMFURIUS, P., BEVERS, E.M., ZWAAL, R.F.A. (1988)

Cytoskeletal degradation by calpain regulates transbilayer movement of phospholipids in the activated platelet plasma membrane. Proceedings of the 29th Dutch Federation Meeting, April 6-7, Uirecht, The Netherlands. Abstract no. 430. Utrecht, published by the Dutch Foundation "Federation of Medical Scientific Societies".

VERHALLEN, P.F.J., COMFURIUS, P., BEVERS, E.M., ZWAAL, R.F.A. (1988)

Cytoskeletal degradation by calpain regulates transbilayer movement of phospholipids in the activated platelet plasma membrane. Proceedings of the 14th International Congress of Biochemistry, July 10-15, Prague, Czechaslovakia Vol.II Abstract no. TU-127. Prague, Videopress IOI. 


\section{Curriculum vitae}

De schrijver van dit proefschrift werd geboren te Maastricht op 16 november 1955. Na het behalen van het diploma Gymnasium-B aan het Stedelijk Lyceum te Maastricht, werd in 1975 aangevangen met de studie scheikunde aan de Rijksuniversiteit Utrecht. In juni 1977 trad hij in het huwelijk met Sylvia Roomberg. In maart 1980 werd het kandidaatsexamen scheikunde met bijvak biologie afgelegd. In januari 1983 werd hij vader van een zoon. In july 1984 werd het doctoraal examen afgelegd, met als specialisatie biochemie (Dr. R.A. Demel) en als bijvak moleculaire neurobiologie (Prof.Dr. W.H. Gispen). Het onderzoek gedlaan tijdens het hoofdvak werd gewaardeerd met de stimuleringsprijs 1983 van de subfaculteit scheikunde. In juli 1984 werd hij tevens vader van een tweeling. Van januari 1985 tot en met december 1987 was hij als onderzoekmedewerker in dienst bij Medigon/NWO, en werd het promotie-onderzoek dat ten grondslag ligt aan dit proefschrift verricht bij de vakgroep Biochemie aan de Rijksuniversiteit Limburg onder leiding van Prof. Dr. R.F.A. Zwaal en Dr. E.M. Bevers. Van februari 1988 tot en met juli 1988 was hij als toegevoegd onderzoeker verbonden aan de Rijksuniversiteit Limburg. Vanaf december $1988 \mathrm{zal}$ hij met behulp van een NATO-science fellowship gaan werken bij Prof. Dr. M.C. Scrutton, verbonden aan het Biochemistry Department van King"s College te London, Engeland. 